CERN-PH-TH/2005-036

DCPT-04-100

DESY 05-059

FERMILAB-PUB-05-060-T

IPPP-04-50

KEK Preprint 2005-16

PRL-TH-05/01

SHEP-05-03

SLAC-PUB-11087

\title{
The role of polarized positrons and electrons in revealing fundamental interactions at the Linear Collider
}

\begin{abstract}
G. Moortgat-Pick ${ }^{1,2}$, T. AbE ${ }^{3}$, G. AlexandeR ${ }^{4}$, B. AnAnthanarayan ${ }^{5}$, A.A. Babich ${ }^{6}$,

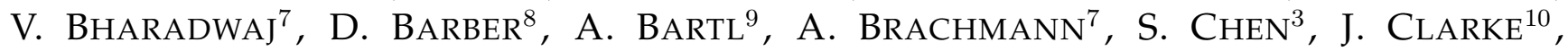
J.E. Clendenin ${ }^{7}$, J. DAinton ${ }^{11}$, K. Desch ${ }^{12}$, M. Diehl ${ }^{8}$, B. Dobos ${ }^{3}$, T. Dorland ${ }^{3}$, H. Eberl ${ }^{13}$, J. Ellis ${ }^{1}$, K. FLÖTtMANN ${ }^{8}$, H. FrAas ${ }^{14}$, F. FrANCO-SOLlOVA ${ }^{8}$, F. FrANKE ${ }^{14}$, A. Freitas ${ }^{15}$, J. GoOdson ${ }^{3}$, J. Gray ${ }^{3}$, A. HAN ${ }^{3}$, S. HeINEMEYer ${ }^{1}$, S. Hesselbach ${ }^{9,16}$, T. Hirose ${ }^{17}$, K. HohenWARTER-SOdeK ${ }^{9}$, J. KAlinOWSKi ${ }^{18}$, T. KernREITER ${ }^{9}$, O. Kittel ${ }^{19}$, S. Kraml ${ }^{1}$, W. Majerotto ${ }^{13}$, A. MartineZ ${ }^{3}$, H.-U. MARTYN ${ }^{20}$, A. Mikhailichenko ${ }^{21}$,

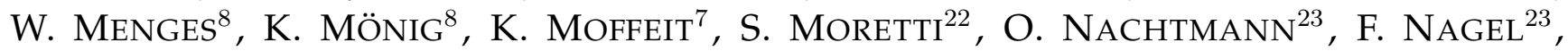
T. NAKANISHi ${ }^{24}$, U. NAUEnberG ${ }^{3}$, T. OMORI ${ }^{25}$, P. OSland ${ }^{1,26}$, A.A. PANKOV ${ }^{6}$, N. PAVER ${ }^{27}$,

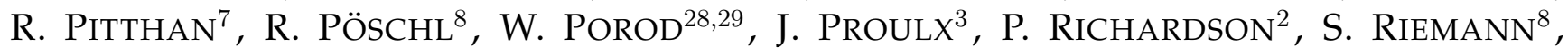
S.D. RINDANI ${ }^{30}$, T.G. RIZZO ${ }^{7}$, P. SCHÜLER ${ }^{8}$, C. SCHWANENBERGER ${ }^{19}$, D. SCOTT ${ }^{10}$,

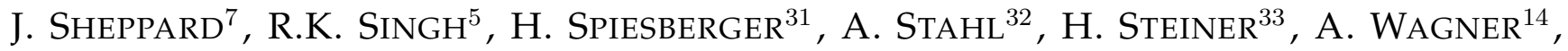
G. WeIGlein ${ }^{2}$, G.W. WILSON ${ }^{34}$, M. WOODS ${ }^{7}$, P. ZERWAS ${ }^{8}$, J. ZHANG ${ }^{3}$, F. ZOMER ${ }^{35}$
\end{abstract}

${ }^{1}$ CERN TH Division, Dept. of Physics, CH-1211 Geneva 23, Switzerland

2 IPPP, University of Durham, Durham DH1 3LE, U.K.

${ }^{3}$ University of Colorado, Boulder, CO 80309-390, USA

${ }^{4}$ University of Tel-Aviv, Tel-Aviv 69978, Israel

${ }^{5}$ Centre for High Energy Physics, Indian Institute of Science, Bangalore, 560012, India

${ }^{6}$ Pavel Sukhoi Technical University, Gomel, 246746, Belarus

7 Stanford Linear Accelerator Center, 2575 Sand Hill Rd, Menlo Park, CA 94025, USA

${ }^{8}$ DESY, Deutsches Elektronen Synchrotron, D-22603 Hamburg, Germany

${ }^{9}$ Institut für Theoretische Physik, Universität Wien, A-1090 Wien, Austria

${ }^{10}$ CCLRC, ASTeC, Daresbury Laboratory, Warrington, Cheshire, WA4 4AD, UK

${ }^{11}$ Dept. of Physics, University of Liverpool, Liverpool L69 7ZE, UK

${ }^{12}$ Phys. Institut, Albert-Ludwigs Universität Freiburg, D-79104 Freiburg, Germany

${ }^{13}$ Inst. f. Hochenergiephysik, Österr. Akademie d. Wissenschaften, A-1050 Wien, Austria

${ }^{14}$ Institut für Theoretische Physik, Universität Würzburg, D-97074 Würzburg, Germany

${ }^{15}$ Fermi National Accelerator Laboratory, Batavia, ILO 60510-0500, USA

${ }^{16}$ High Energy Physics, Uppsala University, Box 535, S-751 21 Uppsala, Sweden

17 Advanced Research Institute for Science and Engineering, Waseda University, 389-5 
Shimooyamada-machi, Machida, Tokyo 194-0202, Japan

${ }^{18}$ Instytut Fizyki Teoretycznej, Uniwersytet Warszawski, PL-00681 Warsaw, Poland

${ }^{19}$ Phys. Institut, Universität Bonn, D-53115 Bonn, Germany

${ }^{20}$ I. Phys. Institut, RWTH Aachen, D-52074 Aachen, Germany

${ }^{21}$ Cornell University, Ithaca, NY 14853, USA

${ }^{22}$ School of Physics and Astronomy, University of Southampton, Highfield, Southampton SO17 1BJ, UK.

${ }^{23}$ Institut für Theoretische Physik, Philosophenweg 16, 69120 Heidelberg, Germany

${ }^{24}$ Dept. of Physics, Nagoya University, Nagoya, Japan.

${ }^{25}$ KEK, Tsukuba-shi, Ibaraki, Japan

${ }^{26}$ Dept. of Physics and Technology, University of Bergen, Allégaten 55, N-5007 Bergen, Norway

${ }^{27}$ Dipartimento di Fisica Teorica, Università di Trieste and Istituto Nazionale di Fisica Nucleare, Sezione di Trieste, Trieste, Italy

${ }^{28}$ Instituto de Física Corpuscular, C. S. I. C. /Universitat Valéncia, E-46071 Valéncia, Spain

${ }^{29}$ Institut für Theoretische Physik, Universität Zürich, Switzerland

30 Theory, Physical Research Laboratory, Navrangpura, Ahmedabad, 380 009, India

${ }^{31}$ Institut für Physik, Johannes-Gutenberg-Universiät Mainz, D-55099 Mainz, Germany

${ }^{32}$ III. Physikalisches Institut, RWTH Aachen, D-52074 Aachen, Germany

33 Dept. of Physics and Lawrence Berkeley Laboratory, University of California, Berkeley, CA 94720, USA

${ }^{34}$ Dept. of Physics and Astronomy, University of Kansas, Lawrence, KS-66045, USA

${ }^{35}$ LAL, Laboratoire de l'Accelerateur Lineaire, Universite de Paris-Sud, F-91405 Cedex Orsay, France

\begin{abstract}
The proposed International Linear Collider (ILC) is well-suited for discovering physics beyond the Standard Model and for precisely unraveling the structure of the underlying physics. The physics return can be maximized by the use of polarized beams. This report shows the paramount role of polarized beams and summarizes the benefits obtained from polarizing the positron beam, as well as the electron beam. The physics case for this option is illustrated explicitly by analyzing reference reactions in different physics scenarios. The results show that positron polarization, combined with the clean experimental environment provided by the linear collider, allows to improve strongly the potential of searches for new particles and the identification of their dynamics, which opens the road to resolve shortcomings of the Standard Model. The report also presents an overview of possible designs for polarizing both beams at the ILC, as well as for measuring their polarization.
\end{abstract}




\section{Contents}

1 Introduction $\quad 8$

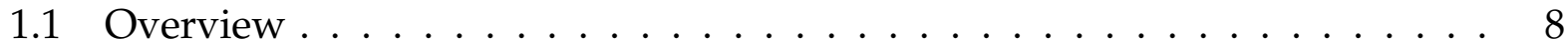

1.2 Polarized cross sections at an $e^{+} e^{-}$collider . . . . . . . . . . . . 10

1.2.1 Formalism . . . . . . . . . . . . . . . . 10

1.2.2 Longitudinally-polarized beams . . . . . . . . . . . . . . . . 13

1.2.3 Use of effective polarization and left-right asymmetry . . . . . . . . 15

1.2.4 Suppression of background in new physics searches . . . . . . . . . . 18

1.3 Importance of electron polarization at the SLC . . . . . . . . . . . . . . 19

2 Open questions of the Standard Model 21

2.1 Top couplings, influence of effective polarization . . . . . . . . . . . . 21

2.2 Standard Model Higgs searches . . . . . . . . . . . . . . . 26

2.2.1 Separation of production processes . . . . . . . . . . . 26

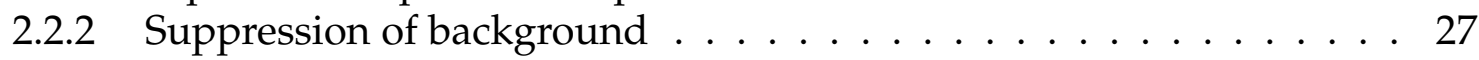

2.2.3 Determination of general $Z Z H$ and $Z \gamma H$ couplings . . . . . . . . . . 28

2.3 Triple gauge boson couplings in $W W$ production . . . . . . . . . . . . . . . . 29

2.3.1 Impact of longitudinally-polarized beams . . . . . . . . . . . . . . . 29

2.3.2 Use of transversely-polarized beams . . . . . . . . . . . . . 33

2.4 Precision electroweak measurements at GigaZ . . . . . . . . . . . . . . . 34

2.4.1 Measurement of $\sin ^{2} \theta_{W}^{\text {eff }}$, application of the Blondel scheme . . . . . . 34

2.4.2 Constraints from $\sin ^{2} \theta_{W}^{\text {eff }}$ on $m_{H}$ and SUSY parameters . . . . . . . . 36

2.4.3 CP violation in 3-jet and 4-jet decays of the Z-boson at GigaZ . . . . 39

3 Searches for new physics with polarized $e^{-}$and $e^{+}$beams 42

3.1 Supersymmetry . . . . . . . . . . . . . . . . . 42

3.1.1 Introduction and choice of SUSY scenarios . . . . . . . . . . . . . 42

3.1.2 Determination of selectron properties . . . . . . . . . . . . 43

3.1.3 Smuon mass measurement above the production threshold . . . . . 48

3.1.4 Determination of third-generation sfermion parameters . . . . . . . 49

3.1.5 Chargino and neutralino production ................. 52

3.1.6 CP violation in neutralino production and decay . . . . . . . . 56

3.1.7 SUSY CP asymmetries with transversely-polarized beams . . . . . . 60

3.1 .8 Non-minimal SUSY models . . . . . . . . . . . . . . . . . . . 63

3.1.9 Production of heavy Higgs bosons in the MSSM . . . . . . . . . . . . 68

3.2 Effective contact-interactions and heavy gauge bosons . . . . . . . . . . . 69

3.2.1 Analysis of four-fermion contact-interactions . . . . . . . . . . 70

3.2.2 Transversely-polarized beams and leptoquark searches . . . . . . . 74 
3.3 Models of gravity in extra dimensions . . . . . . . . . . . . . . . . 77

3.3.1 Direct graviton production . . . . . . . . . . . . . . 77

3.3.2 Signatures of extra dimensions in fermion pair production . . . . . . 80

3.3.3 Use of transversely-polarized beams for graviton searches . . . . . . 82

3.4 Other mechanisms of CP violation . . . . . . . . . . . . . . . 84

3.4.1 Unconventional interactions in $t \bar{t}$ production $\ldots \ldots \ldots . \ldots . \ldots 4$

3.4.2 Transversely-polarized beams for CP violation in $\gamma Z$ production . . 87

4 Summary of the physics case $\quad 91$

5 Machine issues $\quad 95$

5.1 General remarks . . . . . . . . . . . . . . . . . . . . 95

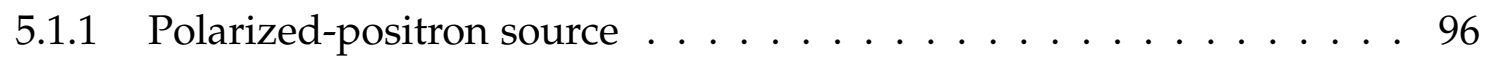

5.1 .2 Polarized electron source . . . . . . . . . . . . . . . . 97

5.1 .3 Polarization preservation . . . . . . . . . . . . 97

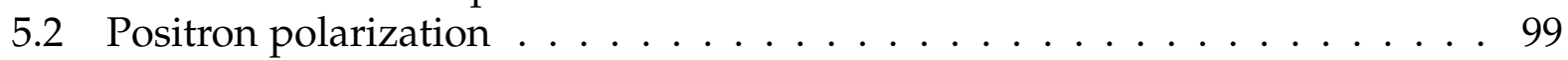

5.2.1 Undulator-based polarized positron source . . . . . . . . . . . 99

5.2.2 Helical undulator development . . . . . . . . . . . . . . . 103

5.2.3 Laser-Compton based polarized positron source . . . . . . . . . . . 104

5.3 Polarized electrons . . . . . . . . . . . . . . . . . . . . . . 109

5.4 Spin manipulation systems . . . . . . . . . . . . . . . . 111

5.5 Polarimetry at the ILC . . . . . . . . . . . . . . . . . . 114

5.5 .1 Compton scattering basics . . . . . . . . . . . . . . . 115

5.5.2 Upstream Compton polarimeter . . . . . . . . . . . . . . . . 116

5.5.3 Downstream Compton polarimeter . . . . . . . . . . . . . 116

5.5.4 Luminosity-weighted polarization . . . . . . . . . . . . . . . 118

5.5 .5 Transverse Polarimetry . . . . . . . . . . . . . . 120

5.5 .6 Future design work . . . . . . . . . . . . . . . . . 120

5.6 Polarization measurements with collider data . . . . . . . . . . . . 121

5.6.1 Measurements with electron polarization only . . . . . . . . . . . . 122

5.6 .2 The Blondel scheme . . . . . . . . . . . . . . . . 123

5.6.3 Experimental aspects . . . . . . . . . . . . . 126

6 Concluding Remarks $\quad 127$

$\begin{array}{ll}\text { A Tools for simulation studies: MC event generators } & 129\end{array}$ 


\section{List of Figures}

1.1 Possible different spin configurations in $e^{+} e^{-}$annihilation $\ldots . . . . . .14$

1.2 Polarization configurations in $s$-channel diagrams . . . . . . . . . . . . . 14

1.3 Polarization configurations in crossed channels . . . . . . . . . . . . . 15

1.4 Single $W$ production $\ldots \ldots \ldots \ldots \ldots \ldots \ldots$

1.5 Effective polarization vs. positron beam polarization . . . . . . . . . . . 17

1.6 Polarization uncertainty in $P_{\text {eff }} \ldots \ldots \ldots \ldots \ldots$

2.1 Anomalous top dipole interaction . . . . . . . . . . . . . . 22

2.2 Flavour-changing neutral coupling in $t \bar{t}$ production . . . . . . . . . . . . . 24

2.3 Feynman diagrams for $e^{+} e^{-} \rightarrow t \bar{q}$ via $Z t q$ or $\gamma t q$ FCNC . . . . . . . . . . . 24

2.4 Higgs production . . . . . . . . . . . . . . . 26

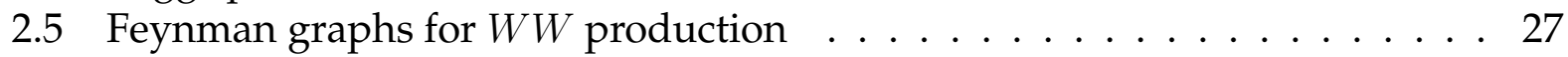

2.6 Feynman graphs for single $W$ production $\ldots \ldots \ldots \ldots \ldots$

2.7 GigaZ: Left-right asymmetry . . . . . . . . . . . . . . . . 36

2.8 GigaZ: $\sin ^{2} \theta_{W}^{\text {eff }}$ vs. $m_{W} \ldots \ldots \ldots \ldots \ldots \ldots \ldots \ldots \ldots \ldots \ldots \ldots \ldots \ldots$

2.9 GigaZ: leptonic effective mixing angle versus $m_{h}$ and $m_{1 / 2} \ldots \ldots$. . . . . 38

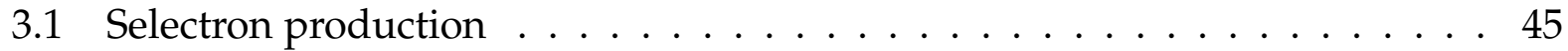

3.2 Test of $\tilde{e}^{ \pm}$quantum numbers . . . . . . . . . . . . . . . . . . 46

3.3 Measurement of $\tilde{e}^{ \pm}$Yukawa couplings . . . . . . . . . . . . . . . . . . . . 47

3.4 Smuon mass measurement: muon energy spectrum . . . . . . . . . . . . . . 49

3.5 Determination of the stau mixing angle . . . . . . . . . . . . . 50

3.6 Production of $\tilde{t}_{1} \tilde{\bar{t}}_{1}$ with polarized beams . . . . . . . . . . . . 51

3.7 Determination of stop parameters via cross sections and $A_{\mathrm{LR}} \ldots \ldots$. . . . 52

3.8 Chargino production . . . . . . . . . . . . . . . 52

3.9 Neutralino production . . . . . . . . . . . . . . . . 53

3.10 Chargino production cross sections with polarized beams . . . . . . . . . 54

3.11 Neutralino production cross section with polarized beams . . . . . . . . . . 54

3.12 T-odd asymmetry for neutralino two-body decays . . . . . . . . . . . . . 57

$3.13 \mathrm{CP}$-odd asymmetry for neutralino decays into tau leptons (I) . . . . . . . . . 58

3.14 CP-odd asymmetry for neutralino decays into tau leptons (II) . . . . . . . . 59

3.15 T-odd asymmetry for neutralino three-body decays . . . . . . . . . . . . . 60

3.16 CP-odd asymmetry for neutralino production with transverse beam polar-

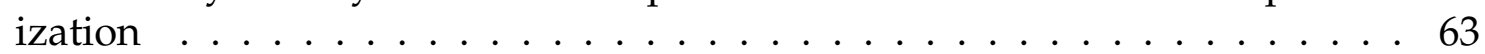

3.17 Singlino-dominated neutralino production . . . . . . . . . . . . 65

3.18 R-parity violation in Bhabha scattering . . . . . . . . . . . . . . . 67

3.19 R-parity-violation in muon production . . . . . . . . . . . . . . . 67

3.20 Sneutrino production in R-parity violating SUSY . . . . . . . . . . . . . . . 68 
3.21 Heavy SUSY Higgs production . . . . . . . . . . . . . . . . 70

3.22 Scale of contact interactions vs. integrated luminosity . . . . . . . . . . 72

3.23 Limits ob contact interaction . . . . . . . . . . . . . . . . 73

$3.2495 \%$ C.L. range for contact-interaction couplings . . . . . . . . . . . . 74

3.25 Determination of $Z^{\prime}$ couplings and masses with polarized beams . . . . . 75

3.26 Limit on chirality-violating couplings with azimuthal asymmetries . . . . . 76

3.27 Graviton production cross sections vs. $M_{D} \ldots \ldots \ldots$. . . . . . . 79

3.28 Differential azimuthal asymmetries in the ADD model . . . . . . . . . . . 83

3.29 Azimuthal distributions in fermion pair production in the ADD model . . . 85

$3.30 S$-couplings in $t \bar{t}$ : Cross section and azimuthal asymmetry vs. cut-off angle 86

3.31 Imaginary part of the coupling $S$ vs. cut-off angle in $t \bar{t}$ production . . . . . . 87

5.1 Possible layout of the polarized positron source . . . . . . . . . . . 100

5.2 Conceptual layout of E166 . . . . . . . . . . . . . . . . . . . . 102

5.3 B-field vs. undulator period . . . . . . . . . . . . . . . . . . . . . . . . . . . . . . . . . . . . . .

5.4 Schematic of wires in helical undulator . . . . . . . . . . . . . . . 104

5.5 Prototype of super-conducting undulator magnets . . . . . . . . . . . . . . . 105

5.6 Undulator design with permanent-magnet design . . . . . . . . . . . . . 105

5.7 Laser-based scheme . . . . . . . . . . . . . . . . . . . . . . . 107

5.8 Compton chamber at the experiment at KEK-ATF . . . . . . . . . . . . . . 107

5.9 Transmission asymmetry . . . . . . . . . . . . . . . . 108

5.10 Apparatus for polarized positron production at KEK-ATF . . . . . . . . . . . 108

5.11 Band gap diagram and transition probabilities for photocathodes . . . . . 110

5.12 Layout of the electron damping ring for serving two IR's . . . . . . . . . 112

5.13 Layout of the positron damping ring for serving two IR's . . . . . . . . . . . . . . 113

5.14 Spin rotation system for transverse polarisation . . . . . . . . . . 113

5.15 Compton cross section . . . . . . . . . . . . . . . . . . 116

5.16 Upstream Compton polarimeter in the TESLA design. . . . . . . . . . . . . 117

5.17 Compton polarimeter system in the extraction line. . . . . . . . . . . . 118

5.18 Vertical distributions and Compton-edge . . . . . . . . . . . . . . 119

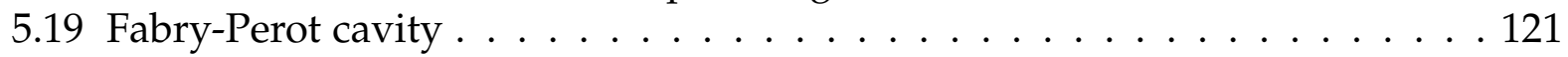

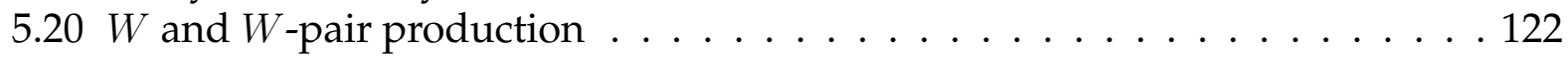

5.21 Left-right asymmetry of WW production . . . . . . . . . . . . 123 


\section{List of Tables}

1.1 Beam polarization and Lorentz structure . . . . . . . . . . . . . . 13

1.2 Bhabha scattering: cross sections with both beams polarized . . . . . . . 15

1.3 Effective polarization and effective luminosity . . . . . . . . . . . 16

1.4 Statistical gain in significance $\ldots \ldots \ldots \ldots$

1.5 Systematic uncertainties at SLD . . . . . . . . . . . . . . 20

2.1 Limits on FCNC in single top production . . . . . . . . . . . . . . 25

2.2 LHC and ILC discovery potential for FCN top couplings . . . . . . . . . 25

2.3 SM Higgs production scaling factors . . . . . . . . . . . . . . . 27

2.4 Scaling factors for $W W$ and $Z Z$ production $\ldots \ldots \ldots \ldots$

2.5 Determination of general Higgs couplings . . . . . . . . . . . . . . . . . . . 29

2.6 CP-conserving triple gauge couplings $\ldots \ldots \ldots \ldots \ldots \ldots$

2.7 Imaginary parts of triple gauge couplings . . . . . . . . . . . . 32

2.8 Simulated sensitivity for triple gauge couplings $\ldots \ldots \ldots \ldots$

2.9 Comparison of electroweak precision observables . . . . . . . . . . . 34

2.10 Sensitivity of CP-violating observables and excited quark mass limits . . . . 41

3.1 Parameters of the chosen SUSY scenarios . . . . . . . . . . . . . . . 44

3.2 WW background in smuon production . . . . . . . . . . . . . 48

3.34 cases of error propagation to production of $\tilde{t}_{1} \tilde{t}_{1}$ with polarized beams . . . 51

3.4 Polarized neutralino cross sections . . . . . . . . . . . . . . . . 55

3.5 T-odd asymmetry for neutralino three-body decays . . . . . . . . . . 60

3.6 Accessible parameter ranges in NMSSM and $\mathrm{E}_{6}$-inspired models . . . . . 64

3.7 Neutralino cross sections in different models . . . . . . . . . . . . . . 66

3.8 Sneutrino production rates vs. Bhabha- and $\mu^{+} \mu^{-}$-scattering $\ldots \ldots \ldots 68$

3.9 Discovery reach for large extra dimensions . . . . . . . . . . . . . . . 79

$3.105-\sigma$ identification reach for the mass scale of extra dimensions . . . . . . 8 84

3.11 Identification and discovery reach for mass scale in ADD model . . . . . . 84

3.12 Limits on $\mathrm{CP}$-violating triple gauge couplings . . . . . . . . . . . 90

4.1 Summary table of physics examples . . . . . . . . . . . . . . . . 94

5.1 Polarized positron parameters . . . . . . . . . . . . . . . . . . 102

5.2 Design parameters of helical undulator prototypes . . . . . . . . . . . . 103

5.3 Comparison of 2 strained superlattice and 1 strained-layer photocathodes. 111

5.4 Compton polarimeter parameters . . . . . . . . . . . . . . . . . 117

5.5 Left-right asymmetry and radiative return . . . . . . . . . . . . . . . . 124

5.6 Relative polarization with Blondel scheme . . . . . . . . . . . . . . . 124

5.7 Relative error on the effective polarization . . . . . . . . . . . . 125 


\section{Chapter 1}

\section{Introduction}

\subsection{Overview}

The first exploration of the TeV energy scale will be made with the proton-proton Large Hadron Collider (LHC) now under construction at CERN, which is scheduled to start operation in the year 2007. Its discovery potential would be complemented by the electronpositron International Linear Collider (ILC) now being designed. The clean signatures and the precise measurements made possible by a high-luminosity linear collider at a known and tunable beam energy could bring revolutionary new insights into our understanding of the fundamental interactions of nature and the structure of matter, space and time $[1,2]$.

The physics return from the investment in a linear collider would be maximized by the possibility of providing polarized beams, particularly because a high degree of polarization can be realized without a significant loss in luminosity. A polarized electron beam would already provide a valuable tool for stringent tests of the Standard Model and for diagnosing new physics. The purpose of this report is to demonstrate that the full potential of the linear collider could be realized only with a polarized positron beam as well. In addition to detailed studies of directly accessible new particles, it would also make possible indirect searches for new physics with high sensitivity in a largely model-independent approach.

In the hunt for physics beyond the Standard Model, only small signs may be visible, and a linear collider (LC) provides optimal conditions for searching for the unexpected. We recall that, in the recent past, the availability of a polarized beam at the SLC (SLAC Linear Collider), the prototype for the ILC, enabled it to compensate in some respects for the fact that it had a lower luminosity than LEP (Large Electron Positron collider). In parallel, polarized lepton scattering has been providing surprising revelations in hadronic structure, and polarized beams play a crucial role in the experimental programmes of RHIC (Relativistic Heavy Ion Collider) as well as HERA (Hadron-Elektron-Ring-Anlage). It is recognized that beam polarization can play an important role in the ILC programme, and polarization of the electron beam is already foreseen for the baseline design [3]. A high degree of at least $80 \%$ polarization is already envisaged, and new results indicate that $90 \%$ should be achievable. The importance of polarizing the positron beam has already been studied in $[1,2,4,5]$ and is discussed as an upgrade option for the ILC [3]. This report focuses on the physics case for choosing this option, as well as reviewing its current technical status. Most of the studies are explicitly evaluated at $\sqrt{s}=500 \mathrm{GeV}$ with an integrated luminosity of $\mathcal{L}_{\text {int }}=500 \mathrm{fb}^{-1}$, which matches the energy reach and annual 
luminosity goal for the first stage of the ILC. The qualitative features of the results with regard to beam polarization, however, are more generally valid also for future energy upgrades from $1 \mathrm{TeV}$ up to multi-TeV energies $[1,2,6]$.

The dominant processes in $e^{+} e^{-}$experiments are annihilation ( $s$-channel) and scattering ( $t$ - and $u$-channel) processes. In annihilation processes, the helicities of the electron and positron are correlated by the spin of the particle(s) exchanged in the direct channel. Suitable combinations of the electron and positron beam polarizations may therefore be used to enhance considerably signal rates and also to efficiently suppress unwanted background processes. These capabilities are particularly welcome in planning searches for new physics, where in many cases only very small rates are predicted. An increased signal/background ratio combined with large luminosity gives additional opportunities for possible discoveries.

On the other hand, in scattering processes, the helicities of the electron and positron can be related directly to the properties of any produced (new) particles. The ability to adjust independently the polarizations of both beams simultaneously will provide unique possibilities for directly probing the properties of the new particles. In particular, it becomes possible to gain direct access to their quantum numbers and chiral couplings with a minimal number of assumptions.

The detailed advantages of polarized beams for many examples of physics both within and beyond the Standard Model are discussed in later sections of this report. Many models of physics beyond the Standard Model have a large number of free parameters.For example, the Minimal Supersymmetric extension of the Standard Model (MSSM) contains more than one hundred new physical parameters, whose complete determination would require many independent experimental observables. Having both beams polarized would increase significantly the number of measurable observables, providing more powerful diagnostic tools, which could be crucial for determining or constraining the many free parameters. The examples show that the combination of two polarized beams may not only be important for the discovery of new particles, but may also be indispensable for revealing the structure of the underlying new physics.

Another prominent example of potential new physics is provided by CP violation. The measured baryon asymmetry of the Universe cannot be explained by the small amount of $\mathrm{CP}$ violation present in the Standard Model. Scenarios for new physics beyond the Standard Model, such as supersymmetry, predict numerous additional sources of CP violation. However, there are tight experimental bounds on CP-violating parameters beyond the Standard Model, and ongoing experiments will strengthen these bounds, constraining severely non-standard sources of $\mathrm{CP}$ violation, or perhaps revealing them. In the quest for CP violation beyond the Standard Model, the simultaneous availability of two polarized beams would offer unique access to powerful CP-odd observables. In particular, transverse beam polarizations would give access to azimuthal asymmetries that can be defined directly in terms of products of final particle momenta, without the need to measure final-state polarizations. For $(V, A)$ interactions, due to the negligible electron mass, observables involving transversely-polarized beams are only available if both beams are polarized.

In addition to direct searches for new physics, the simultaneous polarization of both beams offers new prospects for model-independent indirect searches. Some scales relevant to new physics, such as those characterizing gravity in models with extra dimensions or the compositeness scale of quarks and leptons, could be too large to be directly 
accessible at the energies of future as well as present accelerators. Optimal strategies for indirect searches, as sensitive as possible to small deviations of cross sections from Standard Model predictions due to new physics, may therefore be decisive in these cases. Such searches will undoubtedly take advantage of the clean experimental environment and the high luminosity available at a linear collider. In general, the results may depend strongly on the particular models chosen for analyzing the data. However, the larger class of observables available if both beams are polarized will make it possible to minimize such model dependence.

Even within the Standard Model, having both beams polarized will make it possible to perform tests of unprecedented precision, either at the $\mathrm{Z}$ pole, the WW-threshold or at higher energies. In the GigaZ option the precision on the weak mixing angle can be improved by two orders of magnitude using the left-right asymmetry. This method was pioneered by the SLD experiment at the SLC. Such tests require knowledge of the polarization degree at the per mille-level, which is not possible with conventional polarimetry alone, but may be achieved with the polarization of both beams by applying the Blondel scheme originally proposed for LEP.

In the following, the physics case for positron polarization is presented in detail. Section 1.2 gives an overview of the possible contributing terms in polarized $e^{+} e^{-}$processes. It provides-for the more concerned reader-in the beginning a formalism $[7,8]$ for including longitudinally- as well as transversely-polarized beams. It continues with presenting useful definitions and possible statistical gains in effective polarization, left-right asymmetry measurements and background suppression in searches for new physics are presented. Section 1.3 refers some experimental details from the SLD experiment at the SLC where already highly polarized $e^{-}$beams have been used. In chapter 2 the impact of the polarization of both beams for Standard Model physics at high energy and at the GigaZ option is discussed. Chapter 3 addresses the advantages of polarized beams in direct as well as indirect searches for different kinds of new physics models. The physics results are summarised and the quantitative gain factors are listed in chapter 4 . One may conclude as follows: in the search for unknown new physics that we expect at a linear collider, a polarized positron beam provides important new observables so that two polarized beams could provide decisive tools and that one always gains when using both beams polarized, independent of the direction of physics beyond the Standard Model.

In chapter 5 we also give an overview of the ILC machine considerations for polarized beams, i.e. of sources, spin transport and polarization measurement. The status of designs for both undulator-based and laser-based polarized positron sources are reviewed and compared to that of a conventional unpolarized positron source. It is technically feasible to provide colliding polarized beams with minimal loss in luminosity, and without severe commissioning problems. The precise technical details of these designs will be given in forthcoming technical design reports.

\subsection{Polarized cross sections at an $e^{+} e^{-}$collider}

\subsubsection{Formalism}

The helicity amplitudes for the process

$$
e^{-}\left(p_{e^{-}}, \lambda_{e^{-}}\right) e^{+}\left(p_{e^{+}}, \lambda_{e^{+}}\right) \rightarrow X
$$


can be expressed ${ }^{*}$ as a sum of products of the electron/positron currents $\mathcal{J}_{k}$,

$$
\mathcal{J}_{k}\left(\lambda_{e^{-}}, \lambda_{e^{+}}\right)=\bar{v}\left(p_{e^{+}}, \lambda_{e^{+}}\right) \Gamma_{k} u\left(p_{e^{-}}, \lambda_{e^{-}}\right),
$$

and the contributions $\mathcal{A}_{k}$ of the transition amplitude which depend on the respective final state. In eq. (1.2) $\Gamma_{k}=\left(\gamma_{\mu}, \gamma_{\mu} \gamma_{5}, 1, \gamma_{5}, \sigma_{\mu \nu}\right)$ are the basis elements $(\mathrm{V}, \mathrm{A}, \mathrm{S}, \mathrm{P}, \mathrm{T})$ of the Dirac algebra. The four-component spinors $u, v$ are denoted with the four-momenta $p_{e^{-}}, p_{e^{+}}$in the $e^{-} e^{+}$-c.m.s. and the helicities $\lambda_{e^{-}}, \lambda_{e^{+}}$. In the high-energy limit, the vector and axialvector $(\mathrm{V}, \mathrm{A})$ couple opposite-sign helicities, the scalars $(\mathrm{S}, \mathrm{P})$ and tensors $(\mathrm{T})$ equal-sign helicities.

The helicity amplitude can be written in compact form

$$
F_{\lambda_{e^{-}} \lambda_{e^{+}}}=\bar{v}\left(p_{e^{+}}, \lambda_{e^{+}}\right) \Gamma u\left(p_{e^{-}}, \lambda_{e^{-}}\right),
$$

where for simplification the matrix $\Gamma=\sum \Gamma_{k} \mathcal{A}_{k}$ is introduced.

The polarized electron/positron beam is described by the $2 \times 2$ spin-density matrix $\rho_{\lambda_{e^{ \pm}} \lambda_{e^{ \pm}}^{\prime}}$ so that the transition probability is given by:

$$
\begin{aligned}
|\mathcal{M}|^{2} & =\sum_{\lambda_{e^{-} \lambda_{e^{+}} \lambda_{e}^{\prime}-\lambda_{e^{+}}^{\prime}}} \rho_{\lambda_{e^{-}} \lambda_{e^{-}}^{\prime}} \rho_{\lambda_{e^{+}} \lambda_{e^{+}}^{\prime}} F_{\lambda_{e^{-}} \lambda_{e^{+}}} F_{\lambda_{e^{-}}^{*} \lambda_{e^{+}}^{\prime}}^{*} \\
& =\sum_{\lambda_{e^{-} \lambda_{e}+\lambda_{e^{-}}^{\prime} \lambda_{e^{+}}^{\prime}}} \rho_{\lambda_{e^{-}} \lambda_{e^{-}}^{\prime}} \rho_{\lambda_{e^{+}} \lambda_{e^{+}}^{\prime}} \bar{v}\left(p_{e^{+}}, \lambda_{e^{+}}\right) \Gamma u\left(p_{e^{-}}, \lambda_{e^{-}}\right) \bar{u}\left(p_{e^{-}}, \lambda_{e^{-}}^{\prime}\right) \bar{\Gamma} v\left(p_{e^{+}}, \lambda_{e^{+}}^{\prime}\right)(1.5)
\end{aligned}
$$

where $\bar{\Gamma}=\sum \bar{\Gamma}_{\ell} \mathcal{A}_{\ell}^{*}$ and $\bar{\Gamma}_{\ell}=\gamma^{0} \Gamma_{\ell}^{\dagger} \gamma^{0}$. To evaluate eq. (1.5) helicity projection operators are applied:

$$
\begin{aligned}
u\left(p, \lambda^{\prime}\right) \bar{u}(p, \lambda) & =\frac{1}{2}\left[\delta_{\lambda \lambda^{\prime}}+\gamma_{5} \not^{a} \sigma_{\lambda \lambda^{\prime}}^{a}\right](\not p+m) \\
v\left(p, \lambda^{\prime}\right) \bar{v}(p, \lambda) & =\frac{1}{2}\left[\delta_{\lambda^{\prime} \lambda}+\gamma_{5} \not^{a} \sigma_{\lambda^{\prime} \lambda}^{a}\right](\not p-m),
\end{aligned}
$$

where the Pauli matrices are denoted by $\sigma^{a}, a=1,2,3$. The three four-component spin vectors $s^{a}$ and the momentum $p / m$ form an orthonormal system and can be chosen as

$$
\begin{aligned}
& s^{3 \mu}=\frac{1}{m}(|\mathbf{p}|, E \hat{\mathbf{p}}), \\
& s^{2 \mu}=\left(0, \frac{\mathbf{s}^{3} \times \mathbf{p}_{\text {ref }}}{\left|\mathbf{s}^{3} \times \mathbf{p}_{\text {ref }}\right|}\right), \\
& s^{1 \mu}=\left(0, \frac{\mathbf{s}^{2} \times \mathbf{s}^{3}}{\left|\mathbf{s}^{2} \times \mathbf{s}^{3}\right|}\right),
\end{aligned}
$$

where $\hat{\mathbf{p}}$ is the unit 3-vector in the direction of the respective particle momentum and $\mathbf{p}_{\text {ref }}$ denotes a reference momentum, e.g. of the final state which defines with $\mathbf{p}_{e^{-}}$the scattering plane. The three 4-vectors $\left(s^{1}, s^{2}, s^{3}\right)$ build a right-handed-system and form together with $p / m$ an orthonormal system (for further details see [7]).

In the high-energy limit, $\left(E_{e} \gg m_{e}\right)$, eqn. (1.6)-(1.7), can be written as:

$$
u\left(p_{e^{ \pm}}, \lambda_{e^{ \pm}}^{\prime}\right) \bar{u}\left(p_{e^{ \pm}}, \lambda_{e^{ \pm}}\right)=\frac{1}{2}\left\{\left(1+2 \lambda_{e^{ \pm}} \gamma_{5}\right) \delta_{\lambda_{e \pm} \lambda_{e \pm}^{\prime}}+\gamma_{5}\left[\phi_{e^{ \pm}}^{1} \sigma_{\lambda_{e^{ \pm}} \lambda_{e^{ \pm}}^{\prime}}^{1}+\phi_{e^{ \pm}}^{2} \sigma_{\lambda_{e^{ \pm}} \lambda_{e^{ \pm}}}^{2}\right]\right\} \not p_{e^{ \pm}},
$$

${ }^{*}$ A Fierz transformation may be required. 


$$
v\left(p_{e^{ \pm}}, \lambda_{e^{ \pm}}^{\prime}\right) \bar{v}\left(p_{e^{ \pm}}, \lambda_{e^{ \pm}}\right)=\frac{1}{2}\left\{\left(1-2 \lambda_{e^{ \pm}} \gamma_{5}\right) \delta_{\lambda_{e^{ \pm}}^{\prime} \lambda_{e^{ \pm}}}+\gamma_{5}\left[\oiint_{e^{ \pm}}^{1} \sigma_{\lambda_{e^{ \pm}} \lambda_{e^{ \pm}}}^{1}+\oiint_{e^{ \pm}}^{2} \sigma_{\lambda_{e^{ \pm}} \lambda_{e^{ \pm}}}^{2}\right]\right\} \not p_{e^{ \pm}},
$$

The $2 \times 2$ spin-density matrix of the electron (positron) in eq.(1.5) can be expanded in terms of Pauli matrices $\sigma^{1,2,3}$ :

$$
\rho_{\lambda_{e^{ \pm} \lambda_{e^{ \pm}}^{\prime}}}=\frac{1}{2}\left(\delta_{\lambda_{e^{ \pm} \lambda_{e^{ \pm}}^{\prime}}}+P_{e^{ \pm}}^{1} \sigma_{\lambda_{e^{ \pm}} \lambda_{e^{ \pm}}^{\prime}}^{1}+P_{e^{ \pm}}^{2} \sigma_{\lambda_{e^{ \pm}} \lambda_{e^{ \pm}}^{\prime}}^{2}+P_{e^{ \pm}}^{3} \sigma_{\lambda_{e^{ \pm} \lambda_{e^{ \pm}}^{\prime}}^{3}}^{3}\right) .
$$

Here $P_{e^{ \pm}}^{3}$ (in the following denoted by $P_{e^{ \pm}}$) is the longitudinal degree of polarization with $P_{e^{ \pm}}>0$ (right-handed polarization) if it is parallel to the respective beam direction and $P_{e^{ \pm}}<0$ (left-handed polarization) if it is antiparallel to the beam direction. $P_{e^{ \pm}}^{2}$ is the degree of polarization perpendicular to the scattering plane (spanned by $\mathbf{p}_{e^{-}}$and the reference momentum $\mathbf{p}_{\text {ref }}$ ). $P_{e^{ \pm}}^{1}$ is the degree of transverse polarization in the scattering plane. The signs of the transverse polarizations are chosen with respect to the basis system, eqn.(1.8)-(1.10).

For arbitrarily oriented transverse polarization the components of the transverse polarizations are $P_{e^{ \pm}}^{1}=\mp P_{e^{ \pm}}^{\mathrm{T}} \cos \left(\phi_{ \pm}-\phi\right)$ and $P_{e^{ \pm}}^{2}=P_{e^{ \pm}}^{\mathrm{T}} \sin \left(\phi_{ \pm}-\phi\right)$ where $\phi$ is the azimuthal angle in the c.m.s., $\phi_{+}$and $\phi_{-}$are the azimuthal angles of the $e^{+}$and $e^{-}$polarizations with respect to a fixed coordinate system, e.g. the lab system, and $P_{e^{ \pm}}^{\mathrm{T}}=\sqrt{\left(P_{e^{ \pm}}^{1}\right)^{2}+\left(P_{e^{ \pm}}^{2}\right)^{2}}$ is the degree of the transverse polarization, respectively.

The matrix element squared for a process at an $e^{+} e^{-}$collider with polarized beams can then be written as (cf. also [8,9]):

$$
\begin{aligned}
|\mathcal{M}|^{2}= & \frac{1}{4}\left\{\left(1-P_{e^{-}}\right)\left(1+P_{e^{+}}\right)\left|F_{\mathrm{LR}}\right|^{2}+\left(1+P_{e^{-}}\right)\left(1-P_{e^{+}}\right)\left|F_{\mathrm{RL}}\right|^{2}\right. \\
& +\left(1-P_{e^{-}}\right)\left(1-P_{e^{+}}\right)\left|F_{\mathrm{LL}}\right|^{2}+\left(1+P_{e^{-}}\right)\left(1+P_{e^{+}}\right)\left|F_{\mathrm{RR}}\right|^{2} \\
& -2 P_{e^{-}}^{\mathrm{T}} P_{e^{+}}^{\mathrm{T}}\left\{\left[\cos \left(\phi_{-}-\phi_{+}\right) \operatorname{Re}\left(F_{\mathrm{RR}} F_{\mathrm{LL}}^{*}\right)+\cos \left(\phi_{-}+\phi_{+}-2 \phi\right) \operatorname{Re}\left(F_{\mathrm{LR}} F_{\mathrm{RL}}^{*}\right)\right]\right. \\
& \left.\quad+\left[\sin \left(\phi_{-}+\phi_{+}-2 \phi\right) \operatorname{Im}\left(F_{\mathrm{LR}} F_{\mathrm{RL}}^{*}\right)+\sin \left(\phi_{-}-\phi_{+}\right) \operatorname{Im}\left(F_{\mathrm{RR}}^{*} F_{\mathrm{LL}}\right)\right]\right\} \\
& +2 P_{e^{-}}^{\mathrm{T}}\left\{\cos \left(\phi_{-}-\phi\right)\left[\left(1-P_{e^{+}}\right) \operatorname{Re}\left(F_{\mathrm{RL}} F_{\mathrm{LL}}^{*}\right)+\left(1+P_{e^{+}}\right) \operatorname{Re}\left(F_{\mathrm{RR}} F_{\mathrm{LR}}^{*}\right)\right]\right. \\
& \left.\quad-\sin \left(\phi_{-}-\phi\right)\left[\left(1-P_{e^{+}}\right) \operatorname{Im}\left(F_{\mathrm{RL}}^{*} F_{\mathrm{LL}}\right)-\left(1+P_{e^{+}}\right) \operatorname{Im}\left(F_{\mathrm{RR}}^{*} F_{\mathrm{LR}}\right)\right]\right\} \\
& -2 P_{e^{+}}^{\mathrm{T}}\left\{\cos \left(\phi_{+}-\phi\right)\left[\left(1-P_{e^{-}}\right) \operatorname{Re}\left(F_{\mathrm{LR}} F_{\mathrm{LL}}^{*}\right)+\left(1+P_{e^{-}}\right) \operatorname{Re}\left(F_{\mathrm{RR}} F_{\mathrm{RL}}^{*}\right)\right]\right. \\
& \left.\left.\quad+\sin \left(\phi_{+}-\phi\right)\left[\left(1-P_{e^{-}}\right) \operatorname{Im}\left(F_{\mathrm{LR}}^{*} F_{\mathrm{LL}}\right)-\left(1+P_{e^{-}}\right) \operatorname{Im}\left(F_{\mathrm{RR}}^{*} F_{\mathrm{RL}}\right)\right]\right\}\right\},(1 .
\end{aligned}
$$

where $F_{\mathrm{LL}}$, etc. denote the helicity amlitudes, eq. (1.3), with $\mathrm{L}(\mathrm{R}) \equiv \lambda=-\frac{1}{2}\left(+\frac{1}{2}\right)$.

In the case of circular accelerators [10] one gets (due to the Sokolov-Ternov effect [11]) $\phi_{+}=\phi_{-}+\pi$, whereas at a linear collider $\phi_{-}$and $\phi_{+}$are given by the experimental set-up and can be changed independently.

A few comments may be added to interpret and illustrate the formula (1.14):

- In this expression, the $F^{\prime}$ s contain the dependence of the differential cross section on the polar angle $\theta$ and on $\sqrt{s}$.

- The contributions of different helicity configurations add up incoherently for longitudinally-polarized beams.

- transversely-polarized beams generate interference terms between left- and righthelicity amplitudes. 
In the limit $m_{e} \rightarrow 0$ and within the Standard Model (SM), $F_{\mathrm{RR}}=F_{\mathrm{LL}}=0$, so that if the transverse polarization of the final state particles is not measured, the effects of transverse polarizations are absent in the $\phi$ averaged cross section [9].

In table 1.1 the dependence of the cross section on beam polarization for scalar, pseudoscalar, vector, axial-vector and tensor interactions, $\Gamma=(\mathrm{S}, \mathrm{P}, \mathrm{V}, \mathrm{A}, \mathrm{T})$, are listed for $m_{e} \rightarrow 0$ (see also [12]). In the general case, $m_{e} \neq 0$, all combinations: bilinear, linear, and interference of longitudinal with transverse polarization exist (with the exception of pure $\mathrm{S}$ or $\mathrm{P}$ interactions where no linear polarization dependences occur).

\begin{tabular}{|c|c||c|c|c|c|c|}
\hline \multicolumn{2}{|c|}{ Interaction structure } & \multicolumn{2}{c|}{ Longitudinal } & \multicolumn{2}{c|}{ Transverse } & Longitudinal/Transverse \\
$\Gamma_{k}$ & $\bar{\Gamma}_{\ell}$ & Bilinear & Linear & Bilinear & Linear & Interference \\
\hline $\mathrm{S}$ & $\mathrm{S}$ & $\sim P_{e^{-}} P_{e^{+}}$ & - & $\sim P_{e^{-}}^{T} P_{e^{+}}^{T}$ & - & - \\
$\mathrm{S}$ & $\mathrm{P}$ & - & $\sim P_{e^{ \pm}}$ & $\sim P_{e^{-}}^{T} P_{e^{+}}^{T}$ & - & - \\
$\mathrm{S}$ & $\mathrm{V}, \mathrm{A}$ & - & - & - & $\sim P_{e^{ \pm}}^{T}$ & $\sim P_{e^{ \pm}} P_{e^{\mp}}^{T}$ \\
$\mathrm{~S}$ & $\mathrm{~T}$ & $\sim P_{e^{-}} P_{e^{+}}$ & $\sim P_{e^{ \pm}}$ & $\sim P_{e^{-}}^{T} P_{e^{+}}^{T}$ & - \\
\hline $\mathrm{P}$ & $\mathrm{P}$ & $\sim P_{e^{-}} P_{e^{+}}$ & - & $\sim P_{e^{-}}^{T} P_{e^{+}}^{T}$ & - & - \\
$\mathrm{P}$ & $\mathrm{V}, \mathrm{A}$ & $\sim P_{e^{-}} P_{e^{+}}$ & $\sim P_{e^{ \pm}}$ & $\sim P_{e^{-}}^{T} P_{e^{+}}^{T}$ & $\sim P_{e^{ \pm}}^{T}$ & $\sim P_{e^{ \pm}} P_{e^{\mp}}^{T}$ \\
$\mathrm{P}$ & $\mathrm{T}$ & $\sim P_{e^{-}} P_{e^{+}}$ & $\sim P_{e^{ \pm}}$ & $\sim P_{e^{-}}^{T} P_{e^{+}}^{T}$ & - \\
\hline $\mathrm{V}, \mathrm{A}$ & $\mathrm{V}, \mathrm{A}$ & $\sim P_{e^{-}} P_{e^{+}}$ & $\sim P_{e^{ \pm}}$ & $\sim P_{e^{-}}^{T} P_{e^{+}}^{T}$ & - & - \\
$\mathrm{V}, \mathrm{A}$ & $\mathrm{T}$ & - & - & - & $\sim P_{e^{ \pm}}^{T}$ & $\sim P_{e^{ \pm}} P_{e^{\mp}}^{T}$ \\
\hline $\mathrm{T}$ & $\mathrm{T}$ & $\sim P_{e^{-}} P_{e^{+}}$ & $\sim P_{e^{ \pm}}$ & $\sim P_{e^{-}}^{T} P_{e^{+}}^{T}$ & - & - \\
\hline
\end{tabular}

Table 1.1: Dependence on beam polarization of the transition probability, for (pseudo)scalar-, (axial)vector- and tensor-interactions in the limit $m_{e} \rightarrow 0$.

As can be seen from table 1.1, in pure V,A-interactions for $m_{e} \rightarrow 0$ the effects from transverse beam polarization occur only if both beams are polarized. Effects from transverse beam polarization are particularly interesting in searches for new sources of CP violation [13], see later sections. A key issue for exploiting this option is to use specific differential cross sections and define new CP-odd as well as CP-even asymmetries.

\subsubsection{Longitudinally-polarized beams}

With longitudinally-polarized beams, cross sections at an $e^{+} e^{-}$collider can be subdivided (see fig. 1.1) in [9]:

$$
\begin{aligned}
\sigma_{P_{e^{-}} P_{e^{+}}}= & \frac{1}{4}\left\{\left(1+P_{e^{-}}\right)\left(1+P_{e^{+}}\right) \sigma_{\mathrm{RR}}+\left(1-P_{e^{-}}\right)\left(1-P_{e^{+}}\right) \sigma_{\mathrm{LL}}\right. \\
& \left.+\left(1+P_{e^{-}}\right)\left(1-P_{e^{+}}\right) \sigma_{\mathrm{RL}}+\left(1-P_{e^{-}}\right)\left(1+P_{e^{+}}\right) \sigma_{\mathrm{LR}}\right\},
\end{aligned}
$$

where $\sigma_{\mathrm{RL}}$ stands for the cross section if the $e^{-}$-beam is completely right-handed polarized $\left(P_{e^{-}}=+1\right)$ and the $e^{+}$-beam is completely left-handed polarized $\left(P_{e^{+}}=-1\right)$. the cross sections $\sigma_{\mathrm{LR}}, \sigma_{\mathrm{RR}}$ and $\sigma_{\mathrm{LL}}$ are defined analogously. For partially polarized beams the corresponding measurable cross sections will be denoted as $\sigma_{++}, \sigma_{+-}, \sigma_{-+}, \sigma_{--}$, where the indices give the signs of the absolute electron/positron polarizations $\left|P_{e^{-}}\right|$and $\left|P_{e^{+}}\right|$. 


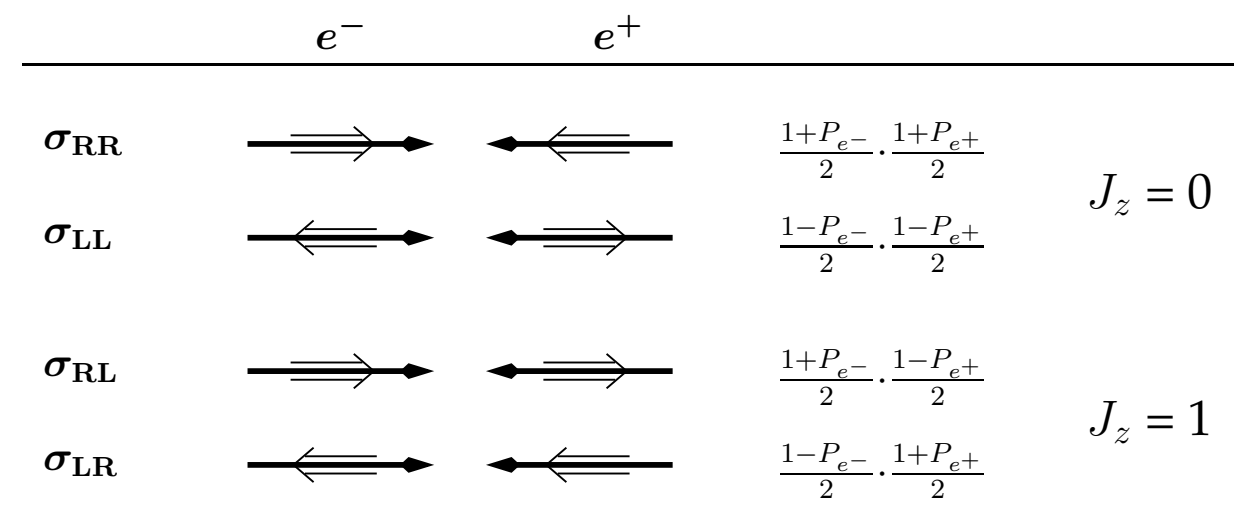

Figure 1.1: The various longitudinal spin configurations in $e^{+} e^{-}$collisions. The thick arrow represents the direction of motion of the particle and the double arrow its spin direction. The first column indicates the corresponding cross section, the fourth column the fraction of this configuration and the last column the total spin projection onto the $e^{+} e^{-}$direction.

One has to distinguish two cases:

a) in annihilation diagrams, see fig. 1.2, the helicities of the incoming beams are coupled to each other;

b) in exchange diagrams, see fig. 1.3, the helicities of the incoming beams are directly coupled to the helicities of the final particles.

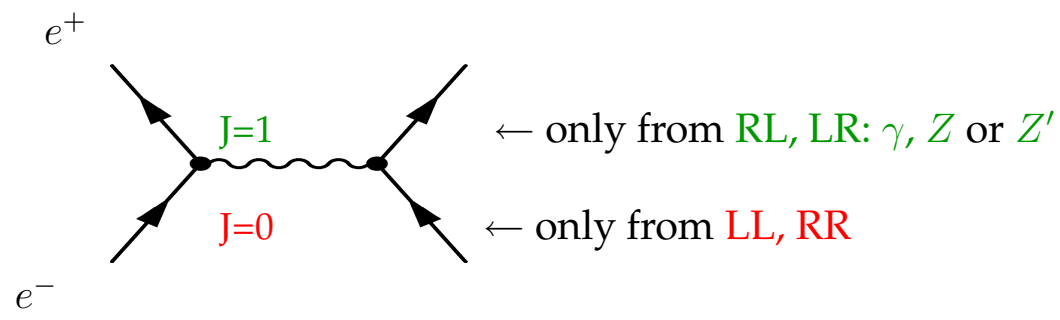

Figure 1.2: Possible configurations in $s$-channel diagrams: the helicities of the incoming $e^{+} e^{-}$beams are directly coupled. Within the Standard Model (SM) only the recombination into a vector particle with $J=1$ is possible, which is given by the LR and RL configurations. New physics (NP) models might contribute to $J=1$ but also to $J=0$, hence the LL or RR configurations.

In case a) within the SM only the recombination into a vector particle with total angular momentum $J=1$ is possible, i.e., the beams have to carry opposite helicities, $F_{\mathrm{LL}}=F_{\mathrm{RR}}=0$. New physics (NP) models can contribute to $J=1$ but might also allow to produce scalar particles, so that also $J=0$ would be allowed, which would result in same-sign helicities of the incoming beams, see fig. 1.2.

In case b) the exchanged particle could be vector, fermion or scalar; the helicity of the incoming particle is directly coupled to the vertex and is independent of the helicity of the second incoming particle. Therefore all possible helicity configurations are in principle possible, see fig. 1.3 . 


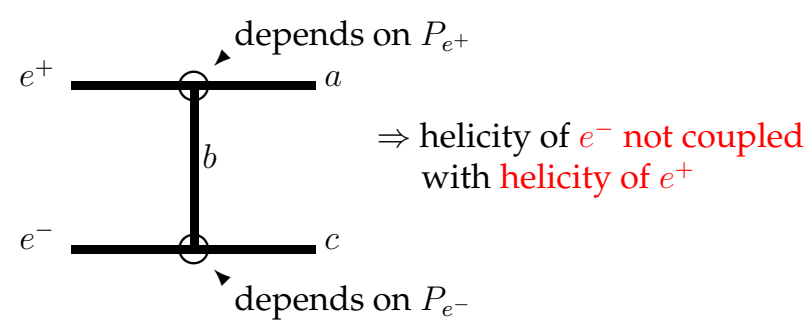

Figure 1.3: Possible configurations in $t$ - and $u$-channel diagrams: the helicity of the incoming beam is directly coupled to the helicity of the final particle and is completely independent of the helicity of the second incoming particle.

SM candidates for case b) are single $W$ production, see fig. 1.4, where the $e^{+} W^{+} \bar{\nu}$ coupling is only influenced by $P_{e^{+}}$, and Bhabha scattering, where the $\gamma, Z$ exchange in the $t$-channel leads to an enhancement of the LL configuration so that the cross sections for the configurations $\left(P_{e^{-}}, P_{e^{+}}\right)=(-80 \%, 0),(-80 \%,+60 \%)$ and $(-80 \%,-60 \%)$ can be of the same order of magnitude, see table 1.2.

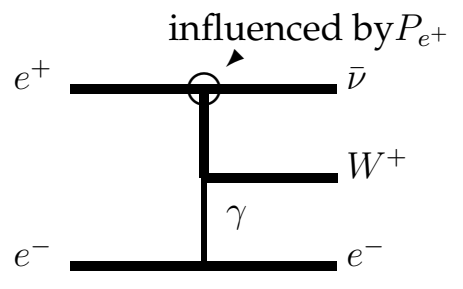

Figure 1.4: Single $W^{+}$production: the vertex $e^{+} W^{+} \bar{\nu}$ depends only on $P_{e^{+}}$.

\begin{tabular}{|l|c|c|c|c|}
\hline$\left(P_{e^{-}}, P_{e^{+}}\right)$ & unpolarized & $(-80 \%, 0)$ & $(-80 \%,-60 \%)$ & $(-80 \%,+60 \%)$ \\
\hline$\sigma\left(e^{+} e^{-} \rightarrow e^{+} e^{-}\right)$ & $4.50 \mathrm{pb}$ & $4.63 \mathrm{pb}$ & $4.69 \mathrm{pb}$ & $4.58 \mathrm{pb}$ \\
\hline
\end{tabular}

Table 1.2: Bhabha scattering at $\sqrt{s}=500 \mathrm{GeV}$ for $45^{\circ}<\theta<135^{\circ}$. Due to the $\gamma, Z$ exchange in the $t$-channel all possible helicity configurations are allowed, e.g. the configuration LL leads to higher cross sections than LR.

\subsubsection{Use of effective polarization and left-right asymmetry}

In the case of $e^{+} e^{-}$annihilation into a vector particle (in the SM this would be $e^{+} e^{-} \rightarrow$ $\gamma / Z^{0}$ ) only the two $J=1$ configurations $\sigma_{\mathrm{RL}}$ and $\sigma_{\mathrm{LR}}$ contribute, as already mentioned in sect. 1.2.2, and the cross section for arbitrary beam polarizations is given by

$$
\begin{aligned}
\sigma_{P_{e^{-}} P_{e^{+}}} & =\frac{1+P_{e^{-}}}{2} \frac{1-P_{e^{+}}}{2} \sigma_{\mathrm{RL}}+\frac{1-P_{e^{-}}}{2} \frac{1+P_{e^{+}}}{2} \sigma_{\mathrm{LR}} \\
& =\left(1-P_{e^{-}} P_{e^{+}}\right) \frac{\sigma_{\mathrm{RL}}+\sigma_{\mathrm{LR}}}{4}\left[1-\frac{P_{e^{-}}-P_{e^{+}}}{1-P_{e^{+}} P_{e^{-}}} \frac{\sigma_{\mathrm{LR}}-\sigma_{\mathrm{RL}}}{\sigma_{\mathrm{LR}}+\sigma_{\mathrm{RL}}}\right] \\
& =\left(1-P_{e^{+}} P_{e^{-}}\right) \sigma_{0}\left[1-P_{\mathrm{eff}} A_{\mathrm{LR}}\right]
\end{aligned}
$$


with

$$
\begin{array}{lrl}
\text { the unpolarized cross section: } & \sigma_{0}=\frac{\sigma_{\mathrm{RL}}+\sigma_{\mathrm{LR}}}{4} \\
\text { the left-right asymmetry: } & A_{\mathrm{LR}}=\frac{\sigma_{\mathrm{LR}}-\sigma_{\mathrm{RL}}}{\sigma_{\mathrm{LR}}+\sigma_{\mathrm{RL}}} \\
\text { and the effective polarization: } & P_{\mathrm{eff}}=\frac{P_{e^{-}}-P_{e^{+}}}{1-P_{e^{+}} P_{e^{-}}}
\end{array}
$$

The collision cross sections can be enhanced if both beams are polarized and if $P_{e^{-}}$and $P_{e^{+}}$have different signs, see eq. (1.16). Introducing the effective luminosity (where the ratio $\mathcal{L}_{\text {eff }} / \mathcal{L}$ reflects the fraction of interacting particles) by

$$
\mathcal{L}_{\text {eff }}=\frac{1}{2}\left(1-P_{e^{-}} P_{e^{+}}\right) \mathcal{L}
$$

eq. (1.16) can be rewritten as:

$$
\sigma_{P_{e^{-}} P_{e^{+}}}=2 \sigma_{0}\left(\mathcal{L}_{\text {eff }} / \mathcal{L}\right)\left[1-P_{\text {eff }} A_{\mathrm{LR}}\right] .
$$

Some values for the effective polarization as well as for the ratio $\mathcal{L}_{\text {eff }} / \mathcal{L}$ are given in table 1.3.

\begin{tabular}{|ll||c|c|}
\hline & & $P_{\text {eff }}$ & $\mathcal{L}_{\text {eff }} / \mathcal{L}$ \\
\hline$P_{e^{-}}=0$, & $P_{e^{+}}=0$ & $0 \%$ & 0.50 \\
$P_{e^{-}}=-100 \%$, & $P_{e^{+}}=0$ & $-100 \%$ & 0.50 \\
$P_{e^{-}}=-80 \%$, & $P_{e^{+}}=0$ & $-80 \%$ & 0.50 \\
$P_{e^{-}}=-80 \%$, & $P_{e^{+}}=+60 \%$ & $-95 \%$ & 0.74 \\
\hline
\end{tabular}

Table 1.3: Effective polarization and effective luminosity, for maximal and realistic values of beam polarization.

The values of the effective polarization can be read off from fig. 1.5. Notice that the effective polarization is closer to $100 \%$ than either of the two beam polarizations. For further references, see $[8,14]$.

In an experiment one would like to extract, e.g., the two quantities $\sigma_{0}$ and $A_{\mathrm{LR}}$, as determined by annihilation into a vector particle, eq. (1.16). This can be done by running the experiment with two different polarization configurations. One would choose one configuration with the electron beam predominantly left-handed and the positron beam right-handed and the second one with both spins reversed. The corresponding cross sections, $\sigma_{-+}$and $\sigma_{+-}$, can be expressed as

$$
\begin{aligned}
& \sigma_{-+}=\frac{1}{4}\left\{\left(1+\left|P_{e^{-}}\right|\left|P_{e^{+}}\right|\right)\left(\sigma_{\mathrm{LR}}+\sigma_{\mathrm{RL}}\right)+\left(\left|P_{e^{-}}\right|+\left|P_{e^{+}}\right|\right)\left(\sigma_{\mathrm{LR}}-\sigma_{\mathrm{RL}}\right)\right\}, \\
& \sigma_{+-}=\frac{1}{4}\left\{\left(1+\left|P_{e^{-}}\right|\left|P_{e^{+}}\right|\right)\left(\sigma_{\mathrm{LR}}+\sigma_{\mathrm{RL}}\right)-\left(\left|P_{e^{-}}\right|+\left|P_{e^{+}}\right|\right)\left(\sigma_{\mathrm{LR}}-\sigma_{\mathrm{RL}}\right)\right\},
\end{aligned}
$$

where the subscripts denote the signs of $P_{e^{-}}$and $P_{e^{+}}$, respectively. It follows that

$$
\sigma_{0}=\frac{\sigma_{-+}+\sigma_{+-}}{2\left(1+\left|P_{e^{+}}\right|\left|P_{e^{-}}\right|\right)}
$$




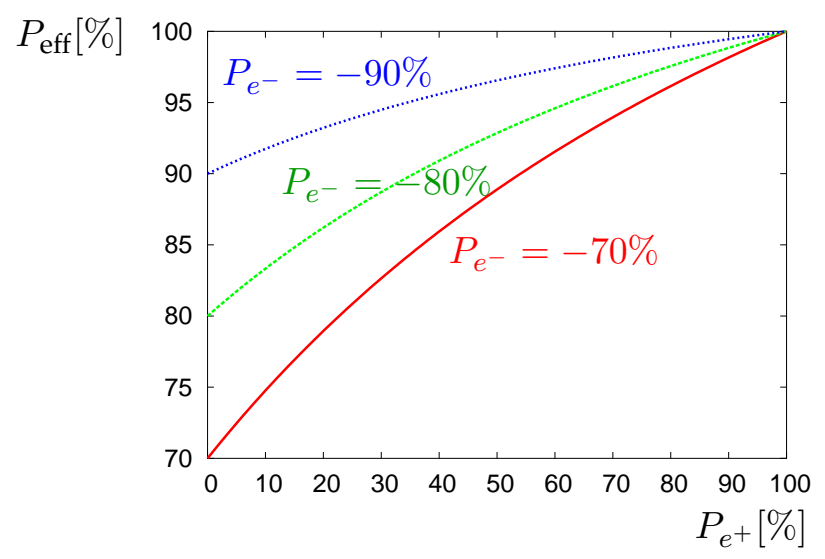

Figure 1.5: Effective polarization vs. positron beam polarization.
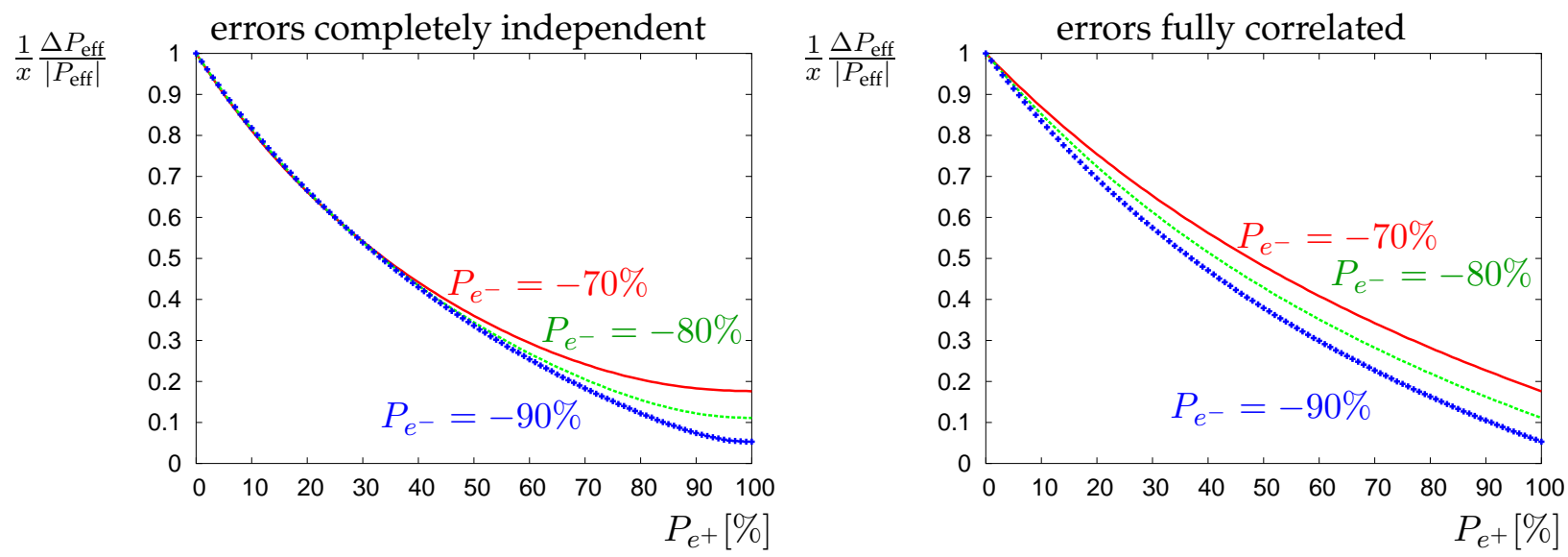

Figure 1.6: Relative uncertainty on the effective polarization, $\Delta P_{\text {eff }} /\left|P_{\text {eff }}\right| \sim \Delta A_{\mathrm{LR}} / A_{\mathrm{LR}}$, normalized to the relative polarimeter precision $x=\Delta P_{e^{-}} / P_{e^{-}}=\Delta P_{e^{+}} / P_{e^{+}}$for independent and correlated errors on $P_{e^{-}}$and $P_{e^{+}}$, see eqs. (1.25), (1.27).

$$
A_{\mathrm{LR}}=\frac{1}{P_{\mathrm{eff}}} A_{L R}^{\mathrm{obs}}=\frac{1}{P_{\mathrm{eff}}} \frac{\sigma_{-+}-\sigma_{+-}}{\sigma_{-+}+\sigma_{+-}}
$$

where $A_{\mathrm{LR}}^{\mathrm{obs}}$ is the measured left-right asymmetry of processes with partially polarized beams.

The contribution of the uncertainty of the polarization measurement to the error in $A_{\mathrm{LR}}$ is, under the assumption that the errors are completely independent and added in quadrature:

$$
\begin{aligned}
\frac{\Delta P_{\text {eff }}}{P_{\text {eff }}} & =\frac{x}{\left(\left|P_{e^{+}}\right|+\left|P_{e^{-}}\right|\right)\left(1+\left|P_{e^{+}}\right|\left|P_{e^{-}}\right|\right)} \sqrt{\left(1-\left|P_{e^{-}}\right|^{2}\right)^{2} P_{e^{+}}^{2}+\left(1-\left|P_{e^{+}}\right|^{2}\right)^{2} P_{e^{-}}^{2}} \\
\left|\frac{\Delta A_{\mathrm{LR}}}{A_{\mathrm{LR}}}\right| & =\left|\frac{\Delta P_{\text {eff }}}{P_{\text {eff }}}\right| .
\end{aligned}
$$

Equal relative precision $x \equiv \Delta P_{e^{-}} / P_{e^{-}}=\Delta P_{e^{+}} / P_{e^{+}}$of the two beam polarizations is assumed.

In the case where the relative errors on $P_{e^{-}}$and $P_{e^{+}}$are fully correlated, like for example by depolarization effects from Bremsstrahlung, the polarization contribution to the 
uncertainty in $A_{\mathrm{LR}}$ is given by the linear sum:

$$
\frac{\Delta P_{\text {eff }}}{P_{\text {eff }}}=\frac{1-\left|P_{e^{+}}\right|\left|P_{e^{-}}\right|}{1+\left|P_{e^{+}}\right|\left|P_{e^{-}}\right|} x
$$

It is immediately obvious from eqs. (1.25) and (1.27), that in both cases $\Delta P_{\text {eff }} / P_{\text {eff }}<$ $\Delta P_{e^{-}} / P_{e^{-}}$. The improvement from positron polarization for the polarization contribution to the error in $A_{\mathrm{LR}}$ is shown in fig. 1.6. The improvement due to positron beam polarization is substantial. For a positron polarization of $60 \%$ the error on $A_{\mathrm{LR}}$ is reduced by a factor of about 3, see also [15].

\subsubsection{Suppression of background in new physics searches}

The use of both beams polarized compared with only electrons polarized can lead to an important gain in statistics and luminosity, i.e. it reduces the required running time. Furthermore, in order to find even small traces of physics beyond the SM and detect new signals, it is important to reduce possible background processes as efficiently as possible. Beam polarization plays an important role in this context by enhancing the signal and suppressing the background rates. It is parametrized by a scaling factor comparing the cross sections with two different polarization configurations a) and b):

$$
\text { scaling factor }=\sigma^{\left(P_{e^{-}}, P_{e^{+}}\right)_{b)}} / \sigma^{\left(P_{e^{-}}, P_{e^{+}}\right)_{a)}} .
$$

Using both beams polarized in configuration $b$ ) instead of just the electron beam polarized in configuration a) can lead to a scaling factor between 0 and at most 2, cf. eq. (1.15).

In cases where the background process depends on beam polarization in a way different from the expected signal process, the gain in using both beams polarized is obvious: suppressing the background and enhancing the signal simultaneously with the suitable polarizaton configuration.

However, also in cases where both processes, background as well as expected signal, show a similar dependence on beam polarization, it is advantageous to use this tool because of the immediate gain in statistical significance. In order to detect a signal,

$$
S=\sigma\left(e^{+} e^{-} \rightarrow X_{1} X_{2}, \ldots\right) \times \mathcal{L}_{\text {int }},
$$

where $X_{1}, X_{2}, \ldots$ are any possible produced particles and $\mathcal{L}_{\text {int }}$ denotes the integrated luminosity, the signal (S) has to be separated from the possible background $(B)$ process(es). The background has a statistical variation of $\sqrt{B}$ (Gaussian distribution assumed, which is suitable thanks to the high statistics at the LC). In order to get a significance of $\mathcal{N}_{\sigma}$ standard deviations for the new signal, it is required that

$$
S>\mathcal{N}_{\sigma} \times \sqrt{B}
$$

Therefore, in order to evaluate the statistical gain correctly when applying both beams polarized, one has to consider not only $S / B$ but also $S / \sqrt{B}$. In table 1.4 the respective values are listed for the two cases where the background and signal processes have the same or an inverse scaling factor. This clearly shows that one gains even in the latter case, since the significance is enhanced! 


\begin{tabular}{|c||c|c||c|c|}
\hline & $S$ & $B$ & $S / B$ & $S / \sqrt{B}$ \\
\hline Example 1 & $\times 2$ & $\times 0.5$ & $\times 4$ & $\times 2 \sqrt{2}$ \\
Example 2 & $\times 2$ & $\times 2$ & Unchanged & $\times \sqrt{2}$ \\
\hline
\end{tabular}

Table 1.4: The gain in 'signal over background', $S / B$, and in significance, $S / \sqrt{B}$, when both beams are polarized compared with the case of polarized electrons only, for two examples where the background and signal processes have the same or an inverse scaling factor.

\subsection{Importance of electron polarization at the SLC}

Before examining, in the next chapters, the physics motivations for polarizing both beams, it is useful to highlight the importance the polarized electron beam had for the SLD experiment at the SLAC Linear Collider (SLC) [16], and to review some of the technical aspects. SLD made detailed and precise measurements of parity violation in weak neutral current interactions by studying $e^{+} e^{-}$collisions at the $Z$ resonance, and utilizing an electron beam with $75 \%$ electron polarization. This degree of electron polarization provided an improvement in statistical power of approximately a factor 25 for many $Z$-pole asymmetry observables, e.g. when using the left-right forward-backward asymmetry, $A_{\mathrm{LR}, \mathrm{FB}}(\cos \theta)$ instead of the forward-backward asymmetry $A_{\mathrm{FB}}(\cos \theta)$. In particular, it allowed the SLD experiment to make the best individual measurement of the weak mixing angle, which is a key ingredient for indirect predictions of the SM Higgs mass. The electron polarization at SLC also provided a powerful tool for bottom quark studies providing a means for tagging $b$ or $\bar{b}$ quark jets, by utilizing the large polarized forward-backward asymmetry in this channel. Moreover, it allowed for studies of parity violation in the $Z b \bar{b}$ vertex.

The flagship measurement for SLD was a high-precision measurement of the left-right asymmetry, and it is useful to recall this measurement in some detail. With the data obtained from $383500 Z$ decays in 1996-98 (about three quarters of the total SLD data sample), the pole value of the asymmetry was measured with high precision, $A_{\mathrm{LR}}^{0}=$ $0.15056 \pm 0.00239$ [17]. The measurement required a precise knowledge of the absolute beam polarization, but did not require the knowledge of absolute luminosity, detector acceptance and efficiency, provided the efficiency for detecting a fermion at some polar angle was equal to the efficiency for detecting an antifermion at the same polar angle.

The beam polarization was measured by a Compton-scattering polarimeter that analyzed the Compton-scattered electrons in a magnetic spectrometer. The accepted energy of the Compton electrons was in the range between 17 and $30 \mathrm{GeV}$. The kinematical minimum was at $17.36 \mathrm{GeV}$. Resolution and spectrometer effects were included and the derived analyzing power of the detector differed by typically about $1 \%$ from the theoretical Compton polarization asymmetry function [18]. Polarimeter data were continuously acquired during the SLC operation [19].

Two additional detectors analyzed the Compton-scattered gammas and assisted in the calibration of the primary spectrometer-based polarimeter. Although they were not used during collision, both provided a useful cross-check for the calibration procedure.

The systematic uncertainties affecting the polarization measurements were a) laser polarization, b) detector linearity, c) analysing power calibration and d) electric noise which led to an uncertainty of $\Delta P_{e} / P_{e} \sim 0.5 \%$, see table 1.5 . 


\begin{tabular}{|l|c|c|c|}
\hline Uncertainty & $\Delta P_{e^{-}} / P_{e^{-}}$ & $\Delta A_{\mathrm{LR}} / A_{\mathrm{LR}}$ & $\Delta A_{\mathrm{LR}}^{0} / A_{\mathrm{LR}}^{0}$ \\
\hline Laser polarization & $0.10 \%$ & & \\
Detector linearity & $0.20 \%$ & & \\
Analysing power calibration & $0.40 \%$ & & \\
Electronic noise & $0.20 \%$ & & \\
Total polarimetry uncertainty & $0.50 \%$ & $0.50 \%$ & \\
Chromaticity and IP corrections & & $0.15 \%$ & \\
Corrections between measured and theoretical $A_{\mathrm{LR}}$ & & $0.07 \%$ & \\
$A_{\mathrm{LR}}$ systematic uncertainty & & $0.52 \%$ & $0.52 \%$ \\
Electroweak interference corrections & & & $0.39 \%$ \\
$A_{\mathrm{LR}}^{0}$ systematic uncertainty & & & $0.64 \%$ \\
\hline
\end{tabular}

Table 1.5: Systematic uncertainties that affect the $A_{\mathrm{LR}}$ measurement. The uncertainty on the electroweak interference correction is caused by the uncertainty on the SLC energy scale, see [17].

Due to chromatic effects (finite beam energy spread, spin precession in the SLC arcs and small chromatic aberrations in the final focus optics) and the large angular divergence of the colliding beams, there is an additional uncertainty in extracting the luminosityweighted beam polarization from the polarimeter measurements. The depolarization due to the $e^{+} e^{-}$interaction itself turned out to be negligible. These effects contribute to an additional $0.15 \%$ uncertainty, see table 1.5 .

There could have been small corrections to the left-right asymmetry extracted by dividing the measured asymmetry by the luminosity-weighted beam polarization. These corrections can arise from left-right asymmetries in luminosity, beam energy, beam polarization, detector backgrounds and detector efficiency. Another potential source of correction is any positron polarization of constant helicity, but this was measured to be negligible. At the SLD experiment these small corrections resulted in an additional $0.07 \%$ uncertainty. The final relative uncertainty for $A_{\mathrm{LR}}$ was $0.52 \%$.

To obtain the pole asymmetry $A_{\mathrm{LR}}^{0}$, the experimental asymmetry $A_{\mathrm{LR}}$ had to be corrected for effects arising from pure photon exchange and $Z \gamma$ interference, where these electroweak corrections sensitively depend on uncertainties in the c.m. energy $\sqrt{s}$. The relative systematic uncertainty for $A_{\mathrm{LR}}^{0}$ was finally around $0.64 \%$, see table 1.5 , and led to the determination of $\sin ^{2} \theta_{W}^{\text {eff }}=0.23107 \pm 0.00030$ from the 1996-98 data sample. Including all of the SLD data sample results in $\sin ^{2} \theta_{W}^{\text {eff }}=0.23097 \pm 0.00027$.

The high-precision measurement of the left-right asymmetry at the $Z$-boson shows the power of the polarized electron beam and also the numerous effects which must be taken into account to achieve such high precision. The SLD experiment has demonstrated how successfully these effects can be controlled. 


\section{Chapter 2}

\section{Open questions of the Standard Model}

\subsection{Top couplings, influence of effective polarization}

Top quark production occurs in the SM via $\gamma, Z$ exchange and the influence of polarized beams can be described by the effective polarization $P_{\text {eff }}$. Availability of both beams polarized allows for a substantial improvement in the $A_{\mathrm{LR}}$ measurement and the determination of couplings and limits for non-standard top physics. Limits on flavour-changing neutral couplings or $\mathrm{CP}$-violating interactions are particularly improved.

The top quark is by far the heaviest fermion observed, yet all the experimental results obtained so far indicate that it behaves as would be expected for a sequential third generation quark. Its large mass, which is close to the scale of electroweak symmetry breaking, makes the top quark a unique object for studying the fundamental interactions in the attometer regime. It is likely to play a key role in pinning down the origin of electroweak symmetry breaking [20]. High precision measurements of the properties of the top quark will be an essential part of the ILC research program.

\section{a) Determination of static electroweak top properties}

A linear collider provides an ideal tool to probe the couplings of the top quark to the electroweak gauge bosons. The neutral electroweak couplings are accessible only at lepton colliders, because top quarks at hadron colliders are pair-produced via gluon exchange.

The most general $(\gamma, Z) t \bar{t}$ couplings can be written as $[2,21,22]$

$$
\Gamma_{t \bar{\gamma} \gamma, Z}^{\mu}=i e\left\{\gamma^{\mu}\left[F_{1 V}^{\gamma, Z}+F_{1 A}^{\gamma, Z} \gamma^{5}\right]+\frac{\left(p_{t}-p_{\bar{t}}\right)^{\mu}}{2 m_{t}}\left[F_{2 V}^{\gamma, Z}+F_{2 A}^{\gamma, Z} \gamma^{5}\right]\right\}
$$

where the only form factors different from zero in the SM are

$$
F_{1 V}^{\gamma}=\frac{2}{3}, \quad F_{1 V}^{Z}=\frac{1}{2 \sin \left(2 \theta_{W}\right)}\left(1-\frac{8}{3} \sin ^{2} \theta_{W}\right), \quad F_{1 A}^{Z}=-\frac{1}{2 \sin \left(2 \theta_{W}\right)} .
$$

The form factor $\left(e / m_{t}\right) F_{2 A}^{\gamma}$ is the CP-violating electric dipole moment, $\left(e / m_{t}\right) F_{2 A}^{Z}$ is the weak electric dipole moment. The factors $\left(e / m_{t}\right) F_{2 V}^{\gamma, Z}$ are the electric and weak magnetic dipole moments. 
Polarization effects have been studied at the top threshold [23]. In the SM the main production process occurs via $\gamma, Z$ exchange and the ratios between the polarized and unpolarized cross sections are given by the scaling factors $\left(1-P_{e^{-}}\right)\left(1+P_{e^{+}}\right)$and $(1+$ $\left.P_{e^{-}}\right)\left(1-P_{e^{+}}\right)$, that can be parametrized with the effective polarization $P_{\text {eff. }}$ To determine the SM top vector coupling, $v_{t}=\left(1-\frac{8}{3} \sin ^{2} \theta_{W}\right)$, one has to measure the left-right asymmetry $A_{\mathrm{LR}}$ with high accuracy. With an integrated luminosity of $\mathcal{L}_{\text {int }}=300 \mathrm{fb}^{-1}$ precisions in $A_{\mathrm{LR}}$ and $v_{t}$ of about $0.4 \%$ and $1 \%$, respectively, can be achieved at the LC. The gain in using simultaneously polarized $e^{-}$and $e^{+}$beams with

$$
\left(P_{e^{-}}, P_{e^{+}}\right)=(\mp 80 \%, \pm 60 \%)
$$

is given by the higher effective polarization of $P_{\text {eff }}=95 \%$ compared to the case of only polarized electrons with $\left|P_{e^{-}}\right|=80 \%$. This leads, according to fig. 1.6 and eqs. (1.25) and (1.26) to a reduction of the relative uncertainty $\Delta A_{\mathrm{LR}} / A_{\mathrm{LR}} \simeq \Delta P_{\text {eff }} / P_{\text {eff }}$ by about a factor of 3 [2].

Limits to all the above mentioned form factors have also been derived in the continuum at $\sqrt{s}=500 \mathrm{GeV}$ for unpolarized beams and $\left(\left|P_{e^{-}}\right|,\left|P_{e^{+}}\right|\right)=(80 \%, 0)[1,2]$. It has been estimated that the polarization of both beams with $\left(\left|P_{e^{-}}\right|,\left|P_{e^{+}}\right|\right)=(80 \%, 60 \%)$ leads to an increase of the $t \bar{t}$ cross section by about a factor $\sim 1.5$ and improves again the bounds by about a factor 3 [2].

\section{b) Limits for CP-violating top dipole couplings}

Measurements aimed at testing top-quark couplings to gauge bosons (both $\mathrm{CP}$-conserving and CP-violating), see eq. 2.1, certainly are an important issue to reveal new fundamental interactions at the linear collider. Searches of anomalous $t \bar{t} \gamma$ and $t \bar{t} Z$ couplings can be made by studying the decay energy and angular distributions of $l^{+}\left(l^{-}\right)$or $b(\bar{b})$ in $e^{+} e^{-} \rightarrow t \bar{t}$ followed by the subsequent decays $t \rightarrow l^{+} \nu_{l} b\left(\bar{t} \rightarrow l^{-} \overline{\nu_{l}} \bar{b}\right)$, as in fig. $2.1[24,25]$.

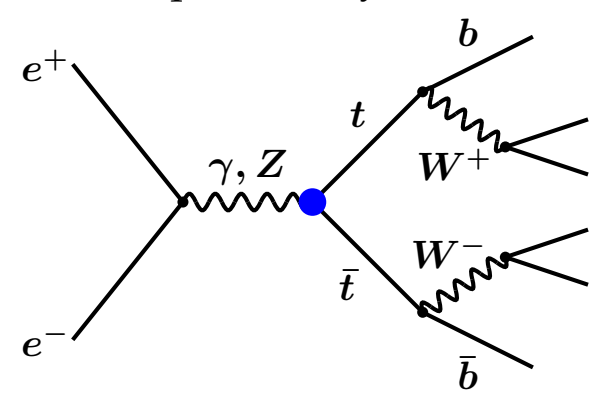

Figure 2.1: Anomalous top dipole interaction.

Particularly interesting are the CP-violating couplings $F_{2 A}^{V}, V=\gamma, Z$, that in the SM can be generated only at the (extremely suppressed) two-loop level. Therefore, detection of the above $\mathrm{CP}$-violating couplings would be a clear manifestation of physics beyond the SM.

In principle, also the $W t b$ vertex can have an anomalous structure, viz.

$$
\Gamma_{W t b}^{\mu}=-\frac{g}{\sqrt{2}} V_{t b}\left[\gamma^{\mu}\left(f_{1}^{\mathrm{L}} P_{\mathrm{L}}+f_{1}^{\mathrm{R}} P_{\mathrm{R}}\right)-\frac{i \sigma^{\mu \nu}\left(p_{t}-p_{b}\right)_{\nu}}{m_{W}}\left(f_{2}^{\mathrm{L}} P_{\mathrm{L}}+f_{2}^{\mathrm{R}} P_{\mathrm{R}}\right)\right],
$$


since in the SM only $f_{1}^{\mathrm{L}}=1$ while all others vanish (in the limit $m_{b} \rightarrow 0$, which is used here). Also, it is assumed that the electron interactions with the gauge bosons are described by the SM.

Focusing on $\mathrm{CP}$ violation, a suitable observable is represented by the forward-backward charge asymmetry [24,25]

$$
\mathcal{A}_{\mathrm{CP}}^{f}\left(P_{e^{-}}, P_{e^{+}}\right)=\frac{\int_{\theta_{0}}^{\pi / 2} d \cos \theta_{f} \frac{d \sigma^{-}}{d \cos \theta_{f}}-\int_{\pi / 2}^{\pi-\theta_{0}} d \cos \theta_{f} \frac{d \sigma^{+}}{d \cos \theta}}{\int_{\theta_{0}}^{\pi / 2} d \cos \theta_{f} \frac{d \sigma^{-}}{d \cos \theta_{f}}+\int_{\pi / 2}^{\pi-\theta_{0}} d \cos \theta_{f} \frac{d \sigma^{+}}{d \cos \theta}},
$$

where $d \sigma^{\mp}$ refer to $f$ and $\bar{f}$ polar angle distribution in the $e^{+}-e^{-}$c.m. frame, respectively (with $f=l, b$ ), and $\theta_{0}$ is a polar angle cut. The asymmetry (2.5) is a genuine measure of $C P$ violation and, actually, for $f=l$ it is sensitive exclusively to the $C P$ violation at the $t \bar{t}$ production vertices. Conversely, for $f=b$ the anomalous structure of $W t b$ in eq. (2.4) appears.

Moreover, the asymmetry (2.5) is defined, and proportional to the CP-violating couplings for all values of beam polarizations, although for $P_{e^{-}} \neq P_{e^{+}}$the initial state is not a $\mathrm{CP}$ eigenstate. This is due to the $s$-channel spin-one exchange character of the production process, that forces only the $\left(e_{\mathrm{L}}^{-} e_{\mathrm{R}}^{+}\right)$or the $\left(e_{\mathrm{R}}^{-} e_{\mathrm{L}}^{+}\right)$components of the initial state to interact with angular momentum conservation.

With $\sqrt{s}=500 \mathrm{GeV}, \mathcal{L}_{\text {int }}=500 \mathrm{fb}^{-1}$, and $60 \%$ reconstruction efficiency for either lepton or $b$, the forward-backward charge asymmetry could be measured at the 5.1- $\sigma(2.4-\sigma)$ level for $b$-quarks (leptons) assuming CP-violating couplings of the order of $5 \times 10^{-2}$ and unpolarized beams. Having both beams $80 \%$ polarized the reach on $\mathcal{A}_{\mathrm{CP}}^{f}$ would even increase up to $16-\sigma(3.5-\sigma)$ and, also, optimal observables and beam polarizations can be adjusted to minimize the statistical error on the determination of the anomalous couplings [24].

Some more CP asymmetries, both in the polar and in the azimuthal angles, can be combined and are studied in [25]. The latter ones, however, require the additional reconstruction of the $t, \bar{t}$ directions. At $\sqrt{s}=500 \mathrm{GeV}, \mathcal{L}_{\text {int }}=500 \mathrm{fb}^{-1}$, the simultaneous $90 \%$ C.L. limits on the CP-violating couplings with $\pm 80 \%$ polarized electrons and unpolarized positrons are of the order of $(1-2) \times 10^{-1}$. Including positron polarization, i.e., $\left(P_{e^{-}}, P_{e^{+}}\right)=( \pm 80 \%, \mp 60 \%)$, the sensitivity can be further improved by $20-30 \%$. ${ }^{*}$ These sensitivities seem not so far from the predictions of some models for new physics, that may be at the level of $10^{-2}-10^{-3}$. Also, they compare favourably with the potential of probing anomalous $t \bar{t} V$ couplings at hadron colliders, see e.g. ref. [26] for the LHC.

\section{c) Limits for flavour-changing neutral top couplings}

Flavour-changing neutral (FCN) couplings of the top quark are relevant to numerous extensions of the SM, and can represent an interesting field for new-physics searches. Limits on top FCN decay branching ratios can be obtained from top-pair production with subsequent $\bar{t}$ decay into $\gamma, Z$ plus light quark governed by the FCN anomalous $t V q$ couplings $(V=\gamma, Z$ and $q=u, c), e^{+} e^{-} \rightarrow t \bar{t} \rightarrow W^{+} b V \bar{q}$, see fig. 2.2, or from single top production $e^{+} e^{-} \rightarrow t \bar{q} \rightarrow W^{+} b \bar{q}$ mediated by the anomalous couplings at the production vertex as

*Individual limits, assuming one non-zero anomalous coupling at a time, are of course substantially more stringent. 
in fig. 2.3. The general expression for the effective-interaction describing the FCN $t V q$ couplings can be written as (effective top couplings to Higgs bosons are not considered in the following) [27]:

$$
\begin{aligned}
-\mathcal{L}= & \frac{g_{W}}{2 c_{W}} X_{t q} \bar{t} \gamma_{\mu}\left(x_{t q}^{\mathrm{L}} P_{\mathrm{L}}+x_{t q}^{\mathrm{R}} P_{\mathrm{R}}\right) q Z^{\mu}+\frac{g_{W}}{2 c_{W}} \kappa_{t q} \bar{t}\left(\kappa_{t q}^{v}-\kappa_{t q}^{a} \gamma_{5}\right) \frac{i \sigma_{\mu \nu} q^{\nu}}{m_{t}} q Z^{\mu} \\
& +e \lambda_{t q} \bar{t}\left(\lambda_{t q}^{v}-\lambda_{t q}^{a} \gamma_{5}\right) \frac{i \sigma_{\mu \nu} q^{\nu}}{m_{t}} q A^{\mu}
\end{aligned}
$$

Here, colour indices are summed over, and the chirality-dependent couplings are normalized as $\left(x_{t q}^{\mathrm{L}}\right)^{2}+\left(x_{t q}^{\mathrm{R}}\right)^{2}=1,\left(\kappa_{t q}^{v}\right)^{2}+\left(\kappa_{t q}^{a}\right)^{2}=1,\left(\lambda_{t q}^{v}\right)^{2}+\left(\lambda_{t q}^{a}\right)^{2}=1$. In the SM, vertices such as those in (2.6) can only be generated at very strongly GIM suppressed loops.

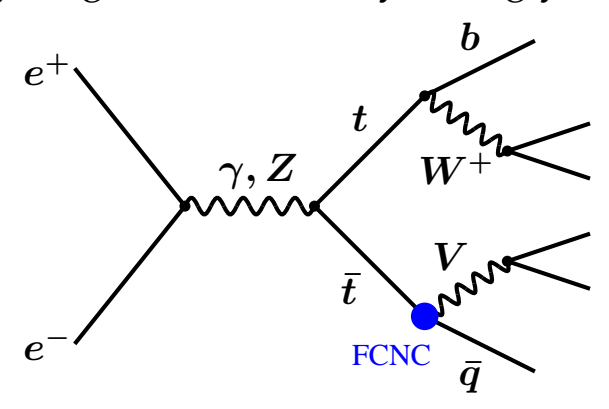

Figure 2.2: Flavour-changing neutral coupling in $t \bar{t}$ production.

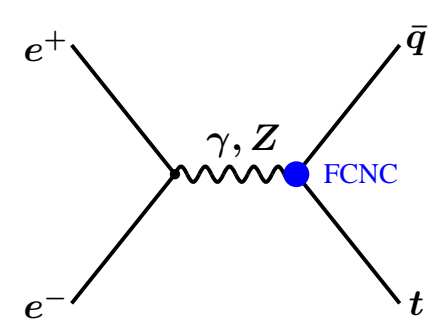

Figure 2.3: Feynman diagrams for $e^{+} e^{-} \rightarrow t \bar{q}$ via $Z t q$ or $\gamma t q$ FCN couplings. The top quark is off-shell and has SM decays.

Single top production is more sensitive to top anomalous couplings but top decays help to disentangle the type of anomalous coupling involved. Beam polarization is very efficient in significantly reducing the background and is therefore particulary important in limits obtained from single top production. The background is essentially dominated by the $W^{+}+2$ jets final state, with $W^{+}$decaying into $l \nu$ and one jet misidentified as a $b$-jet.

With polarization $(80 \%, 0)$, the background decreases by a factor of $1 /\left(1-P_{e^{-}}\right) \approx 5$ while keeping $90 \%$ of the signal. With $(80 \%,-45 \%)$ the background is reduced by a factor of $1 /\left(1-P_{e^{-}}\right)\left(1+P_{e^{+}}\right) \approx 9$ and the signal is increased by $20 \%$ compared to the case of no polarization [27]. In conclusion, $S / B$ and $S / \sqrt{B}$ are improved by factors of 2.1 and 1.7, respectively. ${ }^{\dagger}$

\footnotetext{
${ }^{\dagger}$ For a discussion of these ratios, see sect. 1.2.4.
} 
Already with $e^{-}$and $e^{+}$polarization $(80 \%, 45 \%)$, as an example, one improves the 3- $\sigma$ discovery limits on the vector $\left(\gamma^{\mu}\right)$ coupling at $\sqrt{s}=500 \mathrm{GeV}$ by a factor of 3 (a factor of 1.7 compared to only electron polarization) and the limits on the tensor $\left(\sigma^{\mu \nu}\right)$ coupling at $\sqrt{s}=800 \mathrm{GeV}$ by about a factor 2.6 (a factor 1.8 compared to electron polarization only), see table 2.1.

\begin{tabular}{|l||c|c|c|}
\hline \multicolumn{1}{|c||}{} & unpolarized beams & $\left|P_{e^{-}}\right|=80 \%$ & $\left(\left|P_{e^{-}}\right|,\left|P_{e^{+}}\right|\right)=(80 \%, 45 \%)$ \\
\hline \hline \multicolumn{1}{|c||}{} & $6.1 \times 10^{-4}$ & $\sqrt{s}=500 \mathrm{GeV}$ \\
\hline$B R(t \rightarrow Z q)\left(\gamma_{\mu}\right)$ & $4.8 \times 10^{-5}$ & $3.1 \times 10^{-5}$ & $2.2 \times 10^{-4}$ \\
$B R(t \rightarrow Z q)\left(\sigma_{\mu \nu}\right)$ & $3.0 \times 10^{-5}$ & $1.7 \times 10^{-5}$ & $1.7 \times 10^{-5}$ \\
$B R(t \rightarrow \gamma q)$ & \multicolumn{3}{c|}{$\sqrt{s}=800 \mathrm{GeV}$} \\
\hline \hline \multicolumn{1}{|||}{} & $5.9 \times 10^{-6}$ \\
\hline$B R(t \rightarrow Z q)\left(\gamma_{\mu}\right)$ & $1.7 \times 10^{-5}$ & $4.3 \times 10^{-4}$ & $2.3 \times 10^{-4}$ \\
$B R(t \rightarrow Z q)\left(\sigma_{\mu \nu}\right)$ & $1.3 \times 10^{-5}$ & $7.0 \times 10^{-6}$ \\
$B R(t \rightarrow \gamma q)$ & $1.0 \times 10^{-5}$ & $6.7 \times 10^{-6}$ & $3.6 \times 10^{-6}$ \\
\hline
\end{tabular}

Table 2.1: Single top production: 3- $\sigma$ discovery limits on top flavour changing neutral couplings from top branching fractions at $\sqrt{s}=500 \mathrm{GeV}$ with $\mathcal{L}_{\text {int }}=300 \mathrm{fb}^{-1}$ and at $\sqrt{s}=800 \mathrm{GeV}$ with $\mathcal{L}_{\text {int }}=500 \mathrm{fb}^{-1}$ [27].

A more recent study was made for $\left(\left|P_{e^{-}}\right|,\left|P_{e^{+}}\right|\right)=(80 \%, 60 \%)$ at $\sqrt{s}=500 \mathrm{GeV}$ with $\mathcal{L}_{\text {int }}=345 \mathrm{fb}^{-1}$ and at $\sqrt{s}=800 \mathrm{GeV}$ with $\mathcal{L}_{\text {int }}=534 \mathrm{fb}^{-1}$ including initial state radiation, beamstrahlung and using different kinematical cuts [28]. Comparison with the limits for FNC couplings expected at the LHC shows that the LC measurements are complementary in searches for FCN couplings. Whereas the LHC can be superior in the discovery potential for $\gamma^{\mu}$ couplings, the ILC at $\sqrt{s}=800 \mathrm{GeV}$ with $(80 \%, 60 \%)$ may gain an order of magnitude for the discovery of $\sigma_{\mu \nu}$ couplings to the $Z$ and the photon, see table 2.2. For comparison with the results of full simulation studies for the ATLAS and CMS detector with regard to the LHC discovery potential of FCN couplings, see also [29].

The listed results show that having both beams polarized improves the results concerning measurements of the top properties and enhances considerably the discovery potential for deviations from SM predictions.

Quantitative results: The determination of the top vector coupling is improved by about a factor 3 compared with the case of having only polarized electrons. The limits for top FCN couplings in single top production are improved by about a factor 1.8

\begin{tabular}{|l||c|c|c|}
\hline & LHC & ILC, $\sqrt{s}=500 \mathrm{GeV}$ & ILC, $\sqrt{s}=800 \mathrm{GeV}$ \\
\hline$B R(t \rightarrow Z c)\left(\gamma_{\mu}\right)$ & $3.6 \times 10^{-5}$ & $1.9 \times 10^{-4}$ & $1.9 \times 10^{-4}$ \\
$B R(t \rightarrow Z c)\left(\sigma_{\mu \nu}\right)$ & $3.6 \times 10^{-5}$ & $1.8 \times 10^{-5}$ & $7.2 \times 10^{-6}$ \\
$B R(t \rightarrow \gamma c)$ & $1.2 \times 10^{-5}$ & $1.0 \times 10^{-5}$ & $3.8 \times 10^{-6}$ \\
\hline
\end{tabular}

Table 2.2: 3- $\sigma$ discovery limit on top FCN couplings that can be obtained from top decay processes at the LHC and in single top production at the ILC, $\sqrt{s}=500 \mathrm{GeV}$ and $800 \mathrm{GeV}$ with $\left(P_{e^{-}}, P_{e^{+}}\right)=(80 \%, 60 \%)$ for one year of operation [28]. One anomalous coupling different from zero at a time is assumed. 

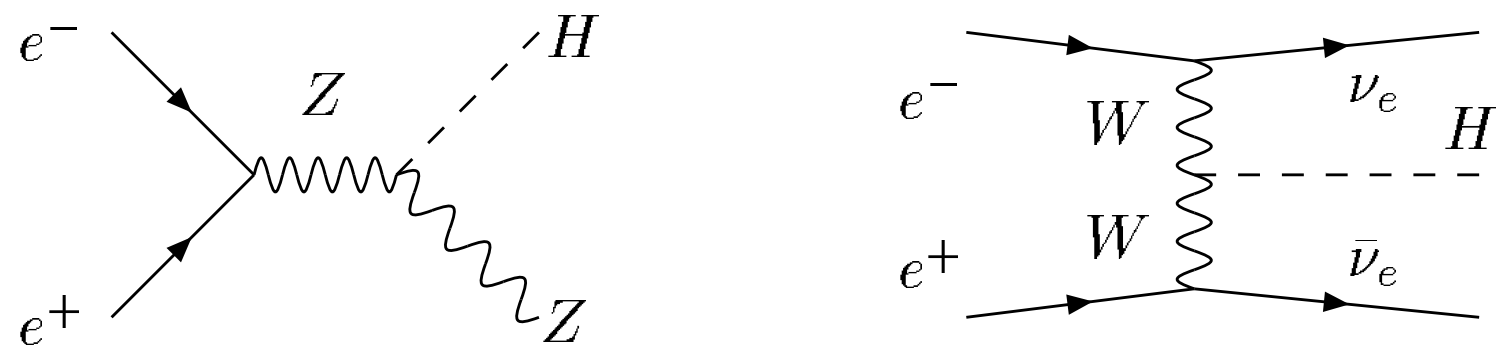

Figure 2.4: Main production mechanism of the SM Higgs boson at the LC

and the sensitivity to CP-violating top couplings is improved by about a factor 1.4 when both beams are longitudinally polarized compared to the case with only polarized electrons, getting closer to predictions from non-standard models.

\subsection{Standard Model Higgs searches}

One of the major physics goals at the ILC is the precise analysis of all the properties of the Higgs particle. For a light Higgs the two major production processes, Higgsstrahlung $e^{+} e^{-} \rightarrow H Z$ and $W W$ fusion $e^{+} e^{-} \rightarrow H \nu \bar{\nu}$, will have similar rates at $\sqrt{s}=500 \mathrm{GeV}$. Beam polarization will be important for background suppression and a better separation of the two processes. Furthermore, the determination of the general Higgs couplings is greatly improved when both beams are polarized.

An accurate study of Higgs production and decay properties can be performed in the clean environment of $e^{+} e^{-}$linear colliders in order to experimentally establish the Higgs mechanism as being responsible for electroweak symmetry breaking [30]. The study of Higgs particles will therefore represent a central part of the ILC physics programme. Beam polarization does not play a key role in determining the Higgs properties; however, it is very helpful for separating the production processes, suppressing the dominant background processes, and improving the accuracy in determining the general couplings. The use of polarized beams is in this context mainly of statistical importance. Since the study of the Higgs properties plays one of the major roles in the ILC programme, we here review the benefits in the Higgs sector of having both beams polarized simultaneously.

\subsubsection{Separation of production processes}

Higgs production at an LC occurs mainly via Higgs-strahlung $e^{+} e^{-} \rightarrow H Z$, fig. 2.4 left, and $W W$ fusion, $e^{+} e^{-} \rightarrow H \nu \bar{\nu}$, fig. 2.4 right. Polarizing both beams enhances the signal and suppresses the background. In table 2.3 the scaling factors, i.e. the ratios of polarized and unpolarized cross sections, are compared for two cases $(1)\left(P_{e^{-}}, P_{e^{+}}\right)=( \pm 80 \%, 0)$, and $(2)\left(P_{e^{-}}, P_{e^{+}}\right)=( \pm 80 \%, \mp 60 \%)$.

If a light Higgs with $m_{H} \leq 130 \mathrm{GeV}$ is assumed, which is the range preferred by both fits of precision observables in the SM [32] and predictions of SUSY theories (see e.g. [33]), Higgs-strahlung dominates for $\sqrt{s} \lesssim 500 \mathrm{GeV}$ and $W W$ fusion for $\sqrt{s} \gtrsim 500 \mathrm{GeV}$. At a LC 


\begin{tabular}{|l||c|c|}
\hline \multicolumn{1}{|l||}{ Configuration } & \multicolumn{2}{c|}{ Scaling factors } \\
$\left(P_{e^{-}}, P_{e^{+}}\right)$ & $e^{+} e^{-} \rightarrow H \nu \bar{\nu}$ & $e^{+} e^{-} \rightarrow H Z$ \\
\hline$(+80 \%, 0)$ & 0.20 & 0.87 \\
$(-80 \%, 0)$ & 1.80 & 1.13 \\
\hline$(+80 \%,-60 \%)$ & 0.08 & 1.26 \\
$(-80 \%,+60 \%)$ & 2.88 & 1.70 \\
\hline
\end{tabular}

Table 2.3: Higgs production scaling factors, eq. (1.28), in the Standard Model at $\sqrt{s}=$ $500 \mathrm{GeV}$ for different polarization configurations with regard to the unpolarized case [5, 31].

with $\sqrt{s}=500 \mathrm{GeV}$ and unpolarized beams, the two processes have comparable cross sections. In the $H \nu_{e} \overline{\nu_{e}}$ final state, there are important contributions from both $H Z$ production and $W W$ fusion. These two contributions exhibit different shapes of the missing mass distributions, which can be exploited to obtain an enriched sample of either process (interference must also be taken into account). Beam polarization can be used to enhance the $H Z$ contribution with respect to the $W W$ fusion signal and vice versa. Table 2.3 shows that there is a gain of a factor $(1.26 / 0.08) /(0.87 / 0.20) \sim 4$ in the ratio $\sigma(H Z) / \sigma(H \nu \bar{\nu})$ when left-handed polarized positrons are used in addition to right-handed polarized electrons. Thus, the relative contribution of $H Z$ and $W W$ fusion can be easily extracted from the missing-mass distribution with two different polarizations without strong model assumptions.

\subsubsection{Suppression of background}

Right-handed electron polarization very efficiently suppresses the background from $W W$ and single $Z$ production via $W W$ fusion, $e^{+} e^{-} \rightarrow Z \nu_{e} \bar{\nu}_{e}$, with $Z$ decaying into fermion pairs. At $\sqrt{s}=500 \mathrm{GeV}$ the latter is important for a light Higgs. For the $W W$ case, fig. 2.5, the suppression can be up to an additional factor 2 if left-handed polarized positrons are also available compared to the case with only right-handed polarized electrons. Positron beam polarization turns out to be also a powerful tool to suppress the $W$ background from single $W$ production, $e^{+} e^{-} \rightarrow W^{-} e^{+} \nu_{e}$ and $e^{+} e^{-} \rightarrow W^{+} e^{-} \bar{\nu}_{e}$, see fig. 2.6.

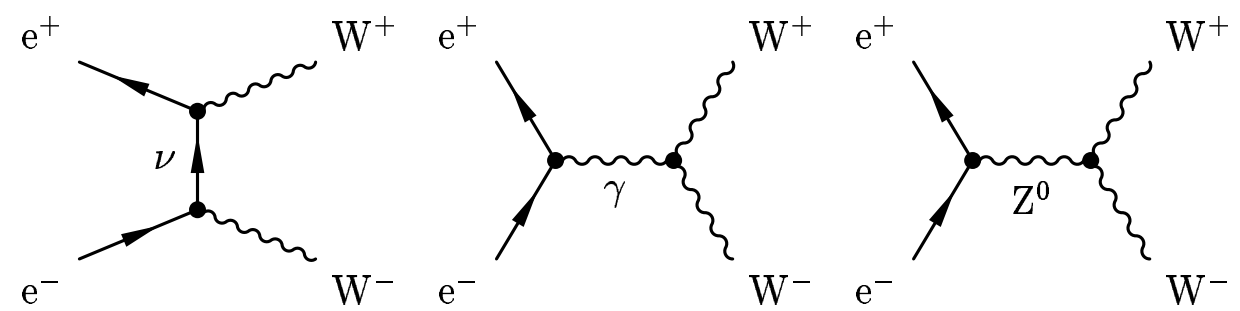

Figure 2.5: Production of $W$ pairs in $\mathrm{e}^{+} \mathrm{e}^{-}$annihilation.

Instead, the advantage of polarization in reducing the $Z Z$ background is rather limited, see table 2.4. However, even in that case where the $S / B$ ratio is only slightly improved, positron polarization in addition to electron polarization (e.g. $\left|P_{e^{-}}\right|=80 \%$ and $\left|P_{e^{+}}\right|=60 \%$ ) improves the statistical significance, see table 1.4. In the case of the $H Z$ 
signal compared to the $Z Z$ background one gains more than $20 \%$ in $S / \sqrt{B}$ when using $\left(P_{e^{-}}, P_{e^{+}}\right)=(+80 \%,-60 \%)$ instead of only $\left(P_{e^{-}}, P_{e^{+}}\right)=(+80 \%, 0)$.
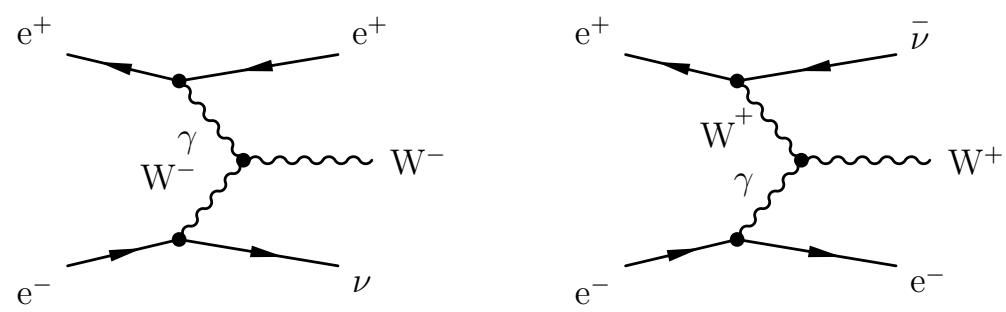

Figure 2.6: Single $W$ production in $\mathrm{e}^{+} \mathrm{e}^{-}$annihilation.

\begin{tabular}{|c|c|c|}
\hline Beam polarization & $e^{+} e^{-} \rightarrow W^{+} W^{-}$ & $e^{+} e^{-} \rightarrow Z Z$ \\
\hline$(+80 \%, 0)$ & 0.20 & 0.76 \\
\hline$(-80 \%, 0)$ & 1.80 & 1.25 \\
\hline \hline$(+80 \%,-60 \%)$ & 0.10 & 1.05 \\
\hline$(-80 \%,+60 \%)$ & 2.85 & 1.91 \\
\hline
\end{tabular}

Table 2.4: Scaling factors, eq. (1.28), of $W W$ and $Z Z$ production at $\sqrt{s}=500 \mathrm{GeV}$ for different polarization configurations with regard to the unpolarized case $[5,31]$.

\subsubsection{Determination of general $Z Z H$ and $Z \gamma H$ couplings}

The accuracy obtainable at the ILC in the determination of the general $Z Z H$ and $Z \gamma H$ couplings, was assessed from $e^{+} e^{-} \rightarrow H Z \rightarrow H f \bar{f}$, by using an optimal-observable method which allows to minimize statistical uncertainties on the couplings, [34,35]. The general effective $H Z V$ interaction Lagrangian considered is parametrized as [36]

$$
\mathcal{L}=\left(1+a_{Z}\right) \frac{g_{Z} m_{Z}}{2} H Z_{\mu} Z^{\mu}+\frac{g_{Z}}{m_{Z}}\left[b_{V} H Z_{\mu \nu} V^{\mu \nu}+c_{V}\left(\partial_{\mu} H Z_{\nu}-\partial_{\nu} H Z_{\mu}\right) V^{\mu \nu}+\tilde{b}_{V} H Z_{\mu \nu} \tilde{V}^{\mu \nu}\right],
$$

where $\tilde{V}^{\mu \nu}=\epsilon^{\mu \nu \alpha \beta} V_{\alpha \beta}$ is the dual of $V^{\mu \nu}=\partial^{\mu} V^{\nu}-\partial^{\nu} V^{\mu}$ and $V=\gamma, Z$. It was shown that beam polarization is essential for determining the sensitivity to the seven general couplings, the CP-even $a_{Z}, b_{Z}, c_{Z}, b_{\gamma}, c_{\gamma}$ and the CP-odd $\tilde{b}_{Z}, \tilde{b}_{\gamma}$ [35]. In particular, to fix the $Z \gamma H$ couplings beam polarization is essential. Simultaneous polarization of the $e^{+}$and $e^{-}$beams results in an increase in the sensitivity, so that for $\sqrt{s}=500 \mathrm{GeV}, \mathcal{L}_{\text {int }}=300 \mathrm{fb}^{-1}$ and $\left(P_{e^{-}}, P_{e^{+}}\right)=( \pm 80 \%, 60 \%)$ the sensitivity is improved by $20-30 \%$ compared to the case of $( \pm 80 \%, 0)[5,34]$, cf. table 2.5 .

Quantitative results: For Higgs masses and c.m. energies where both Higgs-strahlung and $W W$ fusion lead to similar production cross sections, beam polarization is important for the distinction between these processes. The separation is improved by a factor of about 4 with $\left(P_{e^{-}}, P_{e^{+}}\right)=(+80 \%,-60 \%)$, with respect to the case with only right-handed polarized electrons. Furthermore, a factor of 2 can be gained for the $W W$ background suppression. When determining the general $Z V H$ couplings, the sensitivity is increased by about $30 \%$ with respect to the case where only the electrons are polarized and in general limits of the order of $10^{-4}$ can be reached. 


\begin{tabular}{|l||c|c|c|c|}
\hline \multicolumn{1}{|c||}{} & \multicolumn{3}{c|}{$\epsilon_{\tau}=0=\epsilon_{b}$} & $\epsilon_{\tau}=50 \%, \epsilon_{b}=60 \%$ \\
\hline$\left(P_{e^{-}}, P_{e^{+}}\right)$ & $(0,0)$ & $(80 \%, 0)$ & $(80 \%, 60 \%)$ & $(80 \%, 60 \%)$ \\
\hline $\operatorname{Re}\left(b_{Z}\right)$ & 5.5 & 2.8 & 2.3 & 2.2 \\
$\operatorname{Re}\left(c_{Z}\right)$ & 6.5 & 1.4 & 1.1 & 1.1 \\
\hline $\operatorname{Re}\left(b_{\gamma}\right)$ & 123.2 & 5.2 & 3.6 & 3.4 \\
$\operatorname{Re}\left(c_{\gamma}\right)$ & 54.2 & 1.1 & 0.8 & 0.7 \\
\hline $\operatorname{Re}\left(\tilde{b}_{Z}\right)$ & 10.4 & 9.5 & 7.8 & 5.2 \\
$\operatorname{Re}\left(\tilde{b}_{\gamma}\right)$ & 61.8 & 14.5 & 10.1 & 6.3 \\
\hline $\operatorname{Im}\left(b_{Z}-c_{Z}\right)$ & 105.5 & 7.0 & 4.9 & 4.6 \\
$\operatorname{Im}\left(b_{\gamma}-c_{\gamma}\right)$ & 20.6 & 7.0 & 5.7 & 5.4 \\
$\operatorname{Im}\left(\tilde{b}_{Z}\right)$ & 52.1 & 3.2 & 2.2 & 2.2 \\
$\operatorname{Im}\left(\tilde{b}_{\gamma}\right)$ & 10.1 & 3.2 & 2.6 & 2.6 \\
\hline
\end{tabular}

Table 2.5: Determination of general Higgs couplings: Optimal errors in units $\left[10^{-4}\right]$ on general $Z Z \Phi$ and $Z \gamma \Phi$ couplings for different identification efficiencies $\epsilon_{\tau}, \epsilon_{b}$ and beam polarizations $[5,34,35]$.

\subsection{Triple gauge boson couplings in $W W$ production}

\subsubsection{Impact of longitudinally-polarized beams}

In order to test the electroweak gauge group, it is convenient to apply a general parametrization of the gauge-boson self-interactions, which leads to 14 complex parameters, 6 of them CP-violating. Determining all of them in $W W$-pair production represents a strong test of the SM, which predicts only 4 couplings (CP-conserving) to be non-zero. With both beams longitudinally polarized the sensitivity to the different triple gauge couplings is strongly enhanced.

An important feature of the electroweak Standard Model is the non-Abelian nature of its gauge group, which gives rise to gauge boson self-interactions, in particular to the triple gauge couplings (TGCs) $W W \gamma$ and $W W Z$. The most general vertex contains altogether 14 complex parameters, six of them $\mathrm{CP}$-violating. The SM predicts only four $\mathrm{CP}$-conserving real couplings to be non-zero at tree level.

The triple gauge boson vertex $W W V\left(V=Z\right.$ or $\gamma$ and $g_{W W \gamma}=-e, g_{W W Z}=-e \cot \theta_{W}$, $e$ denoting the positron charge and $\theta_{W}$ the Weinberg angle) can be described in the most general form by the effective Lagrangian [37]:

$$
\begin{aligned}
\frac{\mathcal{L}^{W W V}}{i g_{W W V}} & =g_{1}^{V} V^{\mu}\left(W_{\mu \nu}^{-} W^{+\nu}-W_{\mu \nu}^{+} W^{-\nu}\right)-\kappa_{V} W_{\mu}^{-} W_{\nu}^{+} V^{\mu \nu}-\frac{\lambda_{V}}{m_{W}^{2}} V^{\mu \nu} W_{\mu}^{+\rho} W_{\rho \nu}^{-} \\
& +i g_{4}^{V} W_{\mu}^{-} W_{\nu}^{+}\left(\partial^{\mu} V^{\nu}+\partial^{\nu} V^{\mu}\right) \\
& +i g_{5}^{V} \varepsilon^{\mu \nu \rho \sigma}\left[\left(\partial_{\rho} W_{\mu}^{-}\right) W_{\nu}^{+}-W_{\mu}^{-}\left(\partial_{\rho} W_{\nu}^{+}\right)\right] V_{\sigma} \\
& -\frac{\tilde{\kappa}_{V}}{2} W_{\mu}^{-} W_{\nu}^{+} \varepsilon^{\mu \nu \rho \sigma} V_{\rho \sigma}-\frac{\tilde{\lambda}_{V}}{2 m_{W}^{2}} W_{\rho \mu}^{-} W^{+\mu}{ }_{\nu} \varepsilon^{\nu \rho \alpha \beta} V_{\alpha \beta},
\end{aligned}
$$

which is parametrized by seven couplings for each $V$. Their behaviour under the discrete symmetries $\mathrm{C}, \mathrm{P}$ and $\mathrm{CP}$ can be used to divide them into four classes. The three 
couplings $g_{1}^{V}, \kappa_{V}$ and $\lambda_{V}$ conserve $\mathrm{C}$ and $\mathrm{P}$, while $g_{5}^{V}$ violates $\mathrm{C}$ and $\mathrm{P}$ but conserves $\mathrm{CP}$. The couplings $g_{4}^{V}, \tilde{\kappa}_{V}$ and $\tilde{\lambda}_{V}$ violate $\mathrm{CP}$, but $g_{4}^{V}$ conserves $\mathrm{P}$, while $\tilde{\kappa}_{V}$ and $\tilde{\lambda}_{V}$ conserve C. In the SM at tree level the couplings are $g_{1}^{V}=\kappa_{V}=1$, while all others are zero. For convenience one introduces the deviations from the Standard-Model values: $\Delta g_{1}^{\gamma}=g_{1}^{\gamma}-1$, $\Delta g_{1}^{Z}=g_{1}^{Z}-1, \Delta \kappa_{\gamma}=\kappa_{\gamma}-1$ and $\Delta \kappa_{Z}=\kappa_{Z}-1$.

Electromagnetic gauge invariance requires $g_{1}^{\gamma}=1$ and reduces the number of $C$ and $\mathrm{P}$ conserving couplings to five. If one imposed $\mathrm{SU}(2)_{L} \times \mathrm{U}(1)_{Y}$ on eq. (2.8), one would obtain the following relations among the C- and P-conserving couplings, see e.g. [38,39]

$$
\begin{aligned}
\Delta \kappa_{Z} & =-\Delta \kappa_{\gamma} \tan ^{2} \theta_{W}+\Delta g_{1}^{Z} \\
\lambda_{Z} & =\lambda_{\gamma} .
\end{aligned}
$$

This is sometimes used in fits with a reduced number of independent couplings and tests of particular models (not used in the results shown here).

A precision measurement of the TGCs at high energies will be a crucial test of the validity of the SM, given that a variety of new-physics effects can manifest themselves by deviations from the SM predictions (for references, see e.g. [40]). Although no deviation from the SM has been found for the TGCs from LEP data [41], the bounds obtained are comparatively weak. The tightest bounds on the anomalous couplings, i.e., on the differences between couplings and their SM values are of order 0.05 for $\Delta g_{1}^{Z}$ and $\lambda_{\gamma}$, of order 0.1 for $\Delta \kappa_{\gamma}$, and of order 0.1 to 0.6 for the real and imaginary parts of C-and/or P-violating couplings. These numbers correspond to fits where all anomalous couplings, except one, are set to zero. Moreover, many couplings, e.g. the imaginary parts of CP-conserving couplings, have been excluded from the analyses so far.

At a future linear $e^{+} e^{-}$collider one will be able to study these couplings with unprecedented accuracy. A process particularly suitable to study for this purpose is $W$ pair production $e^{+} e^{-} \rightarrow W^{+} W^{-} \rightarrow\left(f_{1} \bar{f}_{2}\right)\left(f_{3} \bar{f}_{4}\right)$, where the final fermions are either leptons or quarks, and where both the $W W \gamma$ and the $W W Z$ couplings can be measured at the c.m.s. energy scale.

\section{Study of TGCs with optimal observables}

In [40] the prospects to measure the full set of 28 (real) TGCs was systematically investigated for unpolarized beams as well as for longitudinal beam polarization, using optimal integrated observables. These observables are constructed to give the smallest possible statistical uncertainties for a given event distribution $[42,43]$. In addition, the abovementioned discrete symmetries are used to simplify the analysis by classifying the TGCs and to test the stability of the results. In $W$ pair production the covariance matrix of these observables consists of four blocks that correspond to CP-even or CP-odd TGCs and to their real or imaginary parts. Within each block all correlations between couplings are taken into account, and simultaneous diagonalization of the covariance matrix allows to treat all 14 couplings at a time and derive separate limits on each one.

The results for the real parts of the CP-conserving TGCs are shown in table 2.6. Only those events where one $W$ boson decays hadronically and the other one into $e \nu$ or $\mu \nu$ are considered due to the favourable reconstruction and branching ratios. In the case of longitudinal polarization, the luminosity is assumed to be equally distributed among both signs and the results are then combined. 


\begin{tabular}{|l|rrrrrrrr|}
\hline$\sqrt{s}=500 \mathrm{GeV}$ & $\operatorname{Re} \Delta g_{1}^{\gamma}$ & $\operatorname{Re} \Delta g_{1}^{Z}$ & $\operatorname{Re} \Delta \kappa_{\gamma}$ & $\operatorname{Re} \Delta \kappa_{Z}$ & $\operatorname{Re} \lambda_{\gamma}$ & $\operatorname{Re} \lambda_{Z}$ & $\operatorname{Re} g_{5}^{\gamma}$ & $\operatorname{Re} g_{5}^{Z}$ \\
\hline No polarization & 6.5 & 5.2 & 1.3 & 1.4 & 2.3 & 1.8 & 4.4 & 3.3 \\
$\left(P_{e^{-}}, P_{e^{+}}\right)=( \pm 80 \%, 0)$ & 3.2 & 2.6 & 0.61 & 0.58 & 1.1 & 0.86 & 2.2 & 1.7 \\
$\left(P_{e^{-}}, P_{e^{+}}\right)=( \pm 80 \%, \mp 60 \%)$ & 1.9 & 1.6 & 0.40 & 0.36 & 0.62 & 0.50 & 1.4 & 1.1 \\
$\left(P_{e^{-}}^{\mathrm{T}}, P_{e^{+}}^{\mathrm{T}}\right)=(80 \%, 60 \%)$ & 2.8 & 2.4 & 0.69 & 0.82 & 0.69 & 0.55 & 2.5 & 1.9 \\
\hline \hline$\sqrt{s}=800 \mathrm{GeV}$ & & & & & & & & \\
\hline No polarization & 4.0 & 3.2 & 0.47 & 0.58 & 1.1 & 0.90 & 3.1 & 2.5 \\
$\left(P_{e^{-}}, P_{e^{+}}\right)=( \pm 80 \%, 0)$ & 1.9 & 1.6 & 0.21 & 0.21 & 0.53 & 0.43 & 1.6 & 1.3 \\
$\left(P_{e^{-}}, P_{e^{+}}\right)=( \pm 80 \%, \mp 60 \%)$ & 1.1 & 0.97 & 0.14 & 0.13 & 0.29 & 0.24 & 0.97 & 0.82 \\
$\left(P_{e^{-}}^{\mathrm{T}}, P_{e^{+}}^{\mathrm{T}}\right)=(80 \%, 60 \%)$ & 1.8 & 1.5 & 0.27 & 0.35 & 0.28 & 0.23 & 1.7 & 1.3 \\
\hline
\end{tabular}

Table 2.6: One- $\sigma$ statistical reach in units of $10^{-3}$ on the real parts of CP-conserving TGCs in the multi-parameter analysis including all anomalous couplings at $\sqrt{s}=500 \mathrm{GeV}$, $\mathcal{L}_{\text {int }}=500 \mathrm{fb}^{-1}$ and $\sqrt{s}=800 \mathrm{GeV}, \mathcal{L}_{\text {int }}=1000 \mathrm{fb}^{-1}$, without and with different beam polarizations [44].

At $800 \mathrm{GeV}$ and an integrated luminosity of $\mathcal{L}=1000 \mathrm{fb}^{-1}$, all uncertainties (with or without polarization) are smaller than for $\sqrt{s}=500 \mathrm{GeV}$ and $500 \mathrm{fb}^{-1}$, reflecting that the anomalous effects in the cross section would be more pronounced at the higher energy. This holds in particular for Re $\Delta \kappa_{\gamma}$. For both c.m. energies the errors on the couplings decrease by about a factor 2 when going from unpolarized beams to longitudinal $e^{-}$polarization and an unpolarized $e^{+}$beam. Going from unpolarized beams to polarized $e^{-}$ and $e^{+}$this gain factor is between 3 and 4 for all couplings, except for $\operatorname{Re} \Delta \kappa_{Z}$ at $800 \mathrm{GeV}$, where it is 4.5 .

It has been emphasized [42] that the following linear combinations can be measured with much smaller correlations than for the parametrization (2.8):

$$
\begin{aligned}
& g_{1}^{\mathrm{L}}=4 \sin ^{2} \theta_{W} g_{1}^{\gamma}+\left(2-4 \sin ^{2} \theta_{W}\right) \xi g_{1}^{Z}, \\
& g_{1}^{\mathrm{R}}=4 \sin ^{2} \theta_{W} g_{1}^{\gamma}-4 \sin ^{2} \theta_{W} \xi g_{1}^{Z},
\end{aligned}
$$

where $\xi=s /\left(s-m_{Z}^{2}\right)$, and similarly for the other couplings. The L and R couplings appear in the amplitudes for left- and right-handed initial $e^{-}$, respectively. Therefore, this parametrization is more 'natural' in the presence of beam polarization than the conventional one of eq. (2.8). For detailed plots showing the sensitivity to the TGCs as a function of the degree of longitudinal polarization, we refer to [40]. There, an extended optimalobservable method [45] has been used, where correlations between TGCs are eliminated through appropriate energy- and polarization-dependent reparametrizations.

For the imaginary parts of the CP-conserving couplings, see table 2.7 , the linear combinations $\tilde{h}_{ \pm}=\operatorname{Im}\left(g_{1}^{\mathrm{R}} \pm \kappa^{\mathrm{R}}\right) / \sqrt{2}$ are used instead of $\operatorname{Im} g_{1}^{\mathrm{R}}$ and $\operatorname{Im} \kappa^{\mathrm{R}}$ [44].

\section{Simulation study of sensitivity to TGCs}

The sensitivity to TGCs for both unpolarized and longitudinally-polarized beams has been simulated in [46], using a spin density matrix method and $\cos \theta_{W}$ distributions, limiting the multi-parameter fit to a restricted number of couplings (in particular, no imaginary parts). Concerning the expected statistics, the cases considered are $\sqrt{s}=500 \mathrm{GeV}$, $\mathcal{L}_{\text {int }}=500 \mathrm{fb}^{-1}$ and $\sqrt{s}=800 \mathrm{GeV}, \mathcal{L}_{\text {int }}=1 \mathrm{ab}^{-1}$. At both energies roughly $4 \times 10^{6}$ 


\begin{tabular}{|c|c|c|c|c|c|c|c|c|}
\hline$\sqrt{s}=500 \mathrm{GeV}$ & $\operatorname{Im} g_{1}^{\mathrm{L}}$ & $\operatorname{Im} \kappa^{\mathrm{L}}$ & $\operatorname{Im} \lambda^{\mathrm{L}}$ & $\operatorname{Im} g_{5}^{\mathrm{L}}$ & $\tilde{h}_{-}$ & $\tilde{h}_{+}$ & $\operatorname{Im} \lambda^{R}$ & $\operatorname{Im} g_{5}^{\mathrm{R}}$ \\
\hline No polarization & 2.7 & 1.7 & 0.48 & 2.5 & 11 & - & 3.1 & 17 \\
\hline$\left(P_{e^{-}}, P_{e^{+}}\right)=(\mp 80 \%, 0)$ & 2.6 & 1.2 & 0.45 & 2.0 & 4.5 & - & 1.4 & 4.3 \\
\hline$\left(P_{e^{-}}, P_{e^{+}}\right)=(\mp 80 \%, \pm 60 \%)$ & 2.1 & 0.95 & 0.37 & 1.6 & 2.5 & - & 0.75 & 2.3 \\
\hline$\left(P_{e^{-}}^{\mathrm{T}}, P_{e^{+}}^{\mathrm{T}}\right)=(80 \%, 60 \%)$ & 2.6 & 1.2 & 0.46 & 2.0 & 3.7 & 3.2 & 0.98 & 4.4 \\
\hline \multicolumn{9}{|l|}{$\sqrt{s}=800 \mathrm{GeV}$} \\
\hline No polarization & 1.5 & 0.74 & 0.18 & 1.5 & 6.0 & - & 1.2 & 9.0 \\
\hline$\left(P_{e^{-}}, P_{e^{+}}\right)=(\mp 80 \%, 0)$ & 1.5 & 0.60 & 0.17 & 1.3 & 2.4 & - & 0.54 & 2.7 \\
\hline$\left(P_{e^{-}}, P_{e^{+}}\right)=(\mp 80 \%, \pm 60 \%)$ & 1.2 & 0.48 & 0.14 & 1.0 & 1.3 & - & 0.29 & 1.4 \\
\hline$\left(P_{e^{-}}^{\mathrm{T}}, P_{e^{+}}^{\mathrm{T}}\right)=(80 \%, 60 \%)$ & 1.5 & 0.60 & 0.17 & 1.3 & 2.1 & 2.0 & 0.39 & 2.8 \\
\hline
\end{tabular}

Table 2.7: Same as table 2.6, but for the imaginary parts and with the L-R parametrization in units $10^{-3}$ [44].

$W$-pair signal events are expected in one or two years runs. As in the previous analysis, the semileptonic $W W \rightarrow q \bar{q} \ell \nu, \ell=e, \mu, \tau$ decay channel was used, and in the polarized beams case the luminosity has been equally shared between the two considered polarization configurations $[(\mp 80 \%, 0)$ for unpolarized positrons and $(-80 \%,+60 \%)$, $(+80 \%,-60 \%)$ for both beams polarized]. Also, for maximal sensitivity and to disentangle $W W Z$ from $W W \gamma$ couplings, the data at both polarizations should be fitted simultaneously.

The Monte Carlo generation of $W^{-} W^{+}$signals included also effects from initial state radiation and beamstrahlung, and $W$-pair generators appropriate to initial polarized beams were used. Other uncertainties that can be relevant to the measurement, such as those on the $W$-mass and on the beam energy, have been taken into account. The background is known from LEP analyses to be very small [47], but in any case it has been fully accounted for. Also simulated detector effects (using the detector simulation program SIMDET [48]) have been included in the unpolarized case in the single-parameter fits of the coupling constants [46].

\begin{tabular}{|cc|rrrrr|rrrr|}
\hline & & $\Delta g_{1}^{Z}$ & $\Delta \kappa_{\gamma}$ & $\lambda_{\gamma}$ & $\Delta \kappa_{Z}$ & $\lambda_{Z}$ & $g_{4}^{Z}$ & $g_{5}^{Z}$ & $\tilde{\kappa}_{Z}$ & $\tilde{\lambda}_{Z}$ \\
\hline $500 \mathrm{GeV}$ & Unpolarized & 3.8 & 0.5 & 1.2 & 0.9 & 1.2 & 8.6 & 2.8 & 6.5 & 1.1 \\
& $\left(\left|P_{e^{-}}\right|,\left|P_{e^{+}}\right|\right)=(80 \%, 0)$ & 2.5 & 0.4 & 0.8 & 0.5 & 0.9 & 8.0 & 2.3 & 5.1 & 1.0 \\
& $\left(\left|P_{e^{-}}\right|,\left|P_{e^{+}}\right|\right)=(80 \%, 60 \%)$ & 1.6 & 0.3 & 0.6 & 0.3 & 0.7 & 4.6 & 1.7 & 3.9 & 0.8 \\
\hline $800 \mathrm{GeV}$ & Unpolarized & 3.9 & 0.3 & 0.5 & 0.5 & 0.5 & 4.2 & 2.9 & 3.0 & 0.5 \\
& $\left(\left|P_{e^{-}}\right|,\left|P_{e^{+}}\right|\right)=(80 \%, 0)$ & 2.2 & 0.2 & 0.5 & 0.3 & 0.5 & 3.2 & 2.4 & 2.4 & 0.4 \\
& $\left(\left|P_{e^{-}}\right|,\left|P_{e^{+}}\right|\right)=(80 \%, 60 \%)$ & 1.3 & 0.2 & 0.3 & 0.2 & 0.3 & 1.8 & 1.4 & 1.4 & 0.3 \\
\hline
\end{tabular}

Table 2.8: Expected sensitivity $\left[10^{-3}\right]$ for the real parts of different couplings at c.m. energies of 500 and $800 \mathrm{GeV}$ and $\mathcal{L}_{\text {int }}=500$ and $1000 \mathrm{fb}^{-1}$, respectively. In the case of polarized beams the luminosity is equally shared between the two combinations $\left(P_{e^{-}}, P_{e^{+}}\right)=( \pm 80 \%, \mp 60 \%)[46]$

The results of this analysis at the generator level for both unpolarized and polarized 
beams are displayed in table 2.8; they are at the level $10^{-3}-10^{-4}$. The numerical results in this table are obtained by allowing only one coupling to vary freely, with all others set to their SM values (one-parameter fit). They show that both beams at the highest degree of polarization can substantially improve the sensitivity expected from electron polarization only. In particular it turns out that most correlations among the different couplings can be suppressed using polarized beams. The correlation can be reduced by about a factor two with $\left(P_{e^{-}}, P_{e^{+}}\right)=( \pm 80 \%, \mp 60 \%)$ compared to $\left(P_{e^{-}}, P_{e^{+}}\right)=( \pm 80 \%, 0)$ derived for a two-dimensional fit at $\sqrt{s}=500 \mathrm{GeV}$. For further comparison with multi-parameter fits and correlation matrix see [46]. A quantitative discussion of the major systematic uncertainties has also been performed. The systematic uncertainty on the measurements of all couplings, that results from varying the experimental beam polarization $\Delta P$ by about $1 \%$, is largely dominant (by a factor up to $5-10$ over the statistical uncertainty, especially at $\sqrt{s}=500 \mathrm{GeV}$ ). This implies that, in order to fully exploit the high statistical sensitivity, the polarization must be known very precisely in order to reduce such systematic uncertainty, say to $\Delta P \sim 0.1 \%-0.2 \%$, so that $\Delta P_{e^{-}} / P_{e^{-}}=0.1-0.2 \%$ and $\Delta P_{e^{+}} / P_{e^{+}}=0.2-0.3$ $\%$ would be needed. Such an experimental accuracy on beam polarizations requires excellent polarimetry or, in case, the application of the Blondel scheme, where polarizations could be derived from polarized cross sections, and with presumably very small correlations with the TGCs. In case of only polarized electrons the beam polarization can be also deduced from the left-right asymmetry from the $W$ production in the forward region (also with rather small correlations with the TGCs), cf. section 5.6.

Quantitative results: With both beams longitudinally polarized the gain in the sensitivity to the triple gauge couplings is up to a factor of 1.8 with respect to the case of only the electron beam polarized.

\subsubsection{Use of transversely-polarized beams}

Specific azimuthal asymmetries with transversely-polarized beams may be crucial for sensitive tests of TGCs. Indeed, the imaginary part of one specific CP-conserving anomalous couplings is accessible with transversely- but not with longitudinally-polarized beams.

It was emphasized in [49] that transversely-polarized beams are important as a tool for studying TGCs and longitudinal $W_{\mathrm{L}}$, in particular for measuring relative phases among helicity amplitudes in $W W$ production.

Optimal observables and multi-parameter analysis have been applied to assess the sensitivity obtainable on TGCs in the case of both electron and positron transversely polarized, and the results are displayed in the bottom lines of tables 2.6 and 2.7 [44]. It turns out that, for most couplings (except $\tilde{h}_{+}$to be discussed separately), the expected uncertainty is approximately of the same size as in the case of only electron longitudinal polarization, but worse than obtained in the case of both beams longitudinally polarized. This situation is common to both c.m. energies $\sqrt{s}=500 \mathrm{GeV}$ and $\sqrt{s}=800 \mathrm{GeV}$.

Consequently, one can conclude that, at least for the c.m. energies and the degrees of polarization considered here, the best sensitivity (and separate limits) on both CP-conserving and CP-violating couplings are obtained by the option of both $e^{-}$and $e^{+}$longitudinally polarized.

The notable exception is represented by $\tilde{h}_{+}$which, as shown in [40], is not measurable 
from the normalized event distribution even with longitudinal polarization. However, with transversely-polarized beams it is possible to measure $\tilde{h}_{+}$with a good sensitivity, see table 2.7 [44]. In the $\gamma-Z$ parametrization, eq. (2.8), this means that the four couplings $\operatorname{Im} g_{1}^{\gamma}, \operatorname{Im} g_{1}^{Z}, \operatorname{Im} \kappa_{\gamma}$ and $\operatorname{Im} \kappa_{Z}$ are not simultaneously measurable without transverse polarization.

In conclusion, although for most anomalous TGCs the longitudinal polarization of both beams is most convenient, the measurement of all possible couplings requires to spend part of the integrated luminosity of the linear collider also in the transverse polarization mode.

Quantitative result: One specific CP-conserving triple gauge coupling, $\tilde{\boldsymbol{h}}_{+}$, is only accessible with both beams transversely polarized. With polarizations $\left(P_{e^{-}}^{\mathrm{T}}, P_{e^{+}}^{\mathrm{T}}\right)=(80 \%$, $60 \%$ ), a one- $\sigma$ statistical uncertainty of about $3.2 \times 10^{-3}$ could be obtained on $\tilde{h}_{+}$at $\sqrt{s}=500 \mathrm{GeV}$.

\subsection{Precision electroweak measurements at GigaZ}

\subsubsection{Measurement of $\sin ^{2} \theta_{W}^{\text {eff }}$, application of the Blondel scheme}

Extremely sensitive tests of the SM can be performed with the help of electroweak precision observables. These can be measured with very high accuracy with the GigaZ option of the ILC, i.e., running with high luminosity at the $Z$-boson resonance. Measuring accurately the left-right asymmetry allows a determination of the effective weak mixing angle $\sin ^{2} \theta_{W}^{\text {eff }}$ with the highest precision. However, in order to exploit the gain in statistics at GigaZ, the relative uncertainties on the beam polarization have to be kept below $0.1 \%$. This ultimate precision cannot be reached with Compton polarimetry, but by using a modified Blondel scheme, which requires both beams polarized.

The GigaZ option refers to running the ILC at the $Z$-boson resonance, yielding about $10^{9} Z$ events in 50-100 days of running, resulting in the most sensitive test of the SM ever made, i.e. determining the electroweak precision observables with an unprecedented precision, see table 2.9 [50].

\begin{tabular}{|c|cccc|}
\hline & SLC/LEP2/Tevatron & Tevatron/LHC & LC & GigaZ/ $W W$ \\
\hline$m_{W}[\mathrm{Mev}]$ & 34 & 15 & 10 & 7 \\
$\sin ^{2} \theta_{W}^{\text {eff }}\left[10^{-5}\right]$ & 16 & $14-20$ & - & 1.3 \\
\hline
\end{tabular}

Table 2.9: Precision of $m_{W}$ and the electroweak mixing angle, $\sin ^{2} \theta_{W}^{\text {eff }}$, compared at present and future experiments [50]. For the measurement of $\sin ^{2} \theta_{W}^{\text {eff }}$ at the GigaZ option the Blondel scheme with $\left(\left|P_{e^{-}}\right|,\left|P_{e^{+}}\right|\right)=(80 \%, 60 \%)$ has been applied [51]. The precision for the $m_{W}$ measurement has been derived in threshold scans, applying both beams polarized with $\left(\left|P_{e^{-}}\right|,\left|P_{e^{+}}\right|\right)=(80 \%, 60 \%)$ [52].

In the SM, the left-right asymmetry $A_{\mathrm{LR}}$ can be written in terms of the effective leptonic electroweak mixing angle:

$$
A_{\mathrm{LR}}=\frac{2\left(1-4 \sin ^{2} \theta_{W}^{\mathrm{eff}}\right)}{1+\left(1-4 \sin ^{2} \theta_{W}^{\mathrm{eff}}\right)^{2}}
$$


The statistical power of the data sample can be fully exploited only when $\Delta A_{\mathrm{LR}}(\mathrm{pol})<$ $\Delta A_{\mathrm{LR}}$ (stat). For $10^{8}-10^{9} Z \mathrm{~s}$ this occurs when $\Delta P_{\text {eff }}<0.1 \%$. In this limit $\Delta \sin ^{2} \theta_{W}^{\text {eff }} \sim 10^{-5}$, which is an order of magnitude smaller than the present value. Thus, it will be crucial to minimize the error in the determination of the polarization.

While improvements in Compton polarimetry achieving a precision $<0.1 \%$ may be difficult to attain, the desired precision should, nevertheless, be possible with the Blondel scheme [54], where the electron and positron polarizations are measured from the polarized cross sections, see also sect. 5.6.2. In this scheme, it is not necessary to know the beam polarization with such extreme accuracy, since $A_{\mathrm{LR}}$ can be directly expressed via cross sections for producing $Z$ s with longitudinally-polarized beams:

$$
\begin{aligned}
\sigma & =\sigma_{\text {unpol }}\left[1-P_{e^{-}} P_{e^{+}}+A_{\mathrm{LR}}\left(P_{e^{+}}-P_{e^{-}}\right)\right] \\
A_{\mathrm{LR}} & =\sqrt{\frac{\left(\sigma_{++}+\sigma_{+-}-\sigma_{-+}-\sigma_{--}\right)\left(-\sigma_{++}+\sigma_{+-}-\sigma_{-+}+\sigma_{--}\right)}{\left(\sigma_{++}+\sigma_{+-}+\sigma_{-+}+\sigma_{--}\right)\left(-\sigma_{++}+\sigma_{+-}+\sigma_{-+}-\sigma_{--}\right)}} .
\end{aligned}
$$

where the cross sections on the RHS have been introduced in sect. 1.2.3. In eqs. (2.12) and (2.13) the absolute polarization values of the left- and right-handed degrees of beam polarization are assumed to be the same. These assumptions have to be checked experimentally by means of polarimetry techniques; since only relative measurements are needed for these measurements, the absolute calibration of the polarimeter cancels and the uncertainty $\Delta A_{\mathrm{LR}}$ is practically independent of $\Delta P_{e^{ \pm}} / P_{e^{ \pm}}[51]$.

It can be seen from (2.13) that the Blondel scheme also requires some luminosity for $\sigma_{++}$and $\sigma_{--}$. However, as shown in figure 2.7 a) only about $10 \%$ of running time will be needed for these combinations to reach the accuracy desired for these high-precision measurements. Fig. $2.7 \mathrm{~b}$ ) shows the statistical error on $A_{\mathrm{LR}}$ as a function of the positron polarization for $P_{e^{-}}=80 \%$. Already with $20 \%$ positron polarization the goal of $\Delta \sin ^{2} \theta_{W}^{\text {eff }}<$ $10^{-4}$ can be reached. For the comparison of different beam polarization configurations and the gain for the $A_{\mathrm{LR}}$ measurements, see also [55].

With the polarization of both beams using the Blondel scheme, i.e. $80 \%$ polarization for electrons and $60 \%$ polarization for positrons, an accuracy of $\Delta \sin ^{2} \theta_{W}^{\text {eff }}=1.3 \times 10^{-5}$ can be achieved in the leptonic final state [51]. If only electron polarization were available, the accuracy would be about $\Delta \sin ^{2} \theta_{W}^{\text {eff }}=9.5 \times 10^{-5}$ [58] if only $\Delta P_{e^{-}} / P_{e^{-}}=0.5 \%$ are achievable. If $P_{e^{-}}=90 \%$ but $\Delta P_{e^{-}} / P_{e^{-}}=0.25 \%$ are assumed, an accuracy of about $\Delta \sin ^{2} \theta_{W}^{\text {eff }}=5 \times 10^{-5}[53]$ may be reachable.

As an example of the potential of the GigaZ $\sin ^{2} \theta_{W}^{\text {eff }}$ measurement, fig. 2.8 [50] compares the present experimental accuracy on $\sin ^{2} \theta_{W}^{\text {eff }}$ and $m_{W}$ from LEP/SLD/Tevatron and the prospective accuracy from the LHC and from the LC without GigaZ option with the predictions of the SM and the MSSM. With GigaZ a very sensitive test of the theory will be possible.

Quantitative results: Compared with the case with only the electron beam polarized and using Compton polarimetry, the effective gain is up to an order of magnitude in the accuracy for measuring $\sin ^{2} \theta_{W}^{\text {eff }}$ with both beams polarized and using the Blondel scheme if $\left(\left|P_{e^{-}}\right|,\left|P_{e^{+}}\right|\right)=(80 \%, 60 \%)$ and $\Delta P_{e^{ \pm}} / P_{e^{ \pm}}=0.5 \%$ are assumed. 

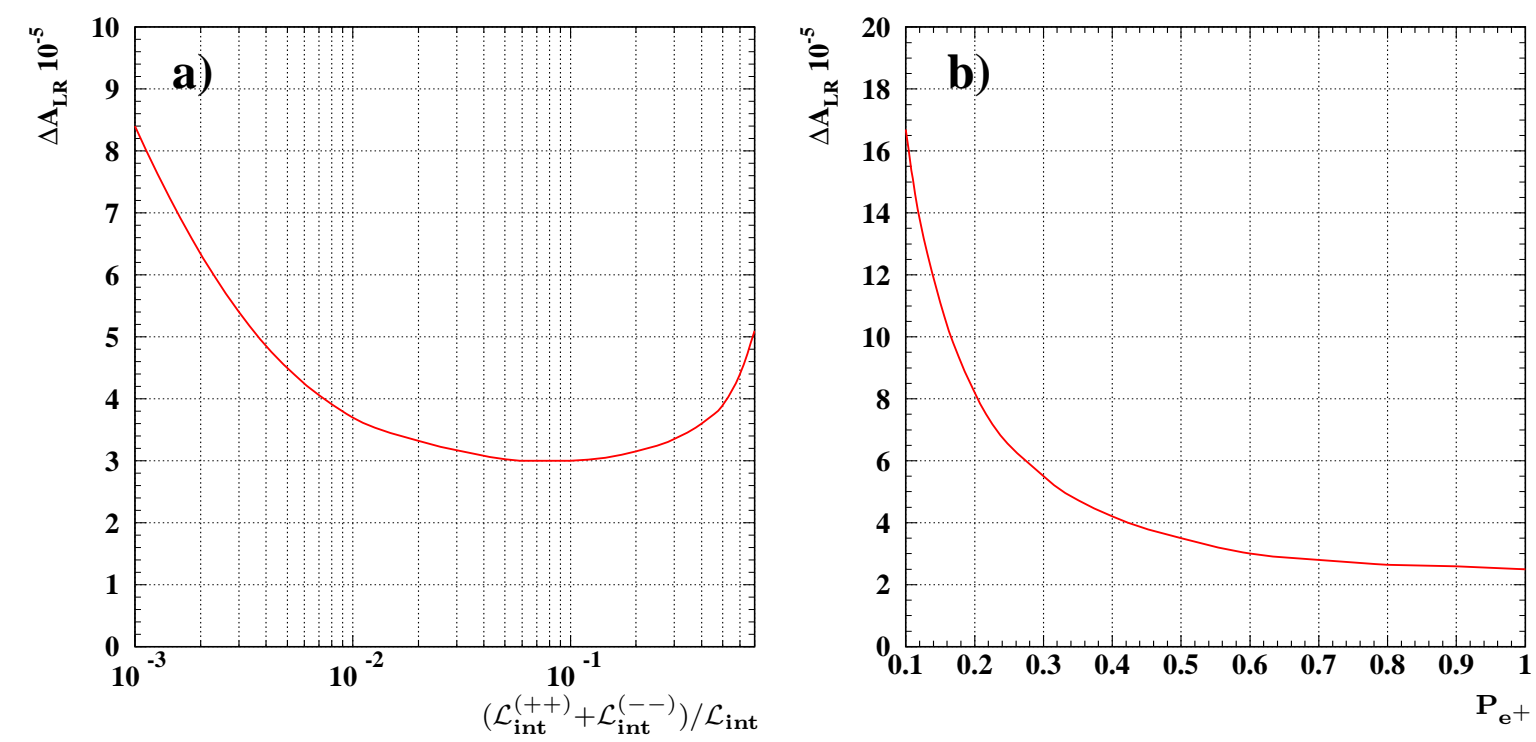

Figure 2.7: Test of the electroweak theory: the statistical error on $A_{\mathrm{LR}}$ of $e^{+} e^{-} \rightarrow Z \rightarrow$ $\ell \bar{\ell}$ at GigaZ, (a) as a function of the fraction of luminosity spent on the less favoured polarization combinations $\sigma_{++}$and $\sigma_{--}$and (b) its dependence on $P_{e^{+}}$for fixed $P_{e^{-}}=$ $\pm 80 \%[51]$.

\subsubsection{Constraints from $\sin ^{2} \theta_{W}^{\text {eff }}$ on Higgs-boson masses and SUSY pa- rameters}

Compared with the case where only the electron beam is polarized, the polarization of both beams leads to a gain of about one order of magnitude in the accuracy of the effective weak mixing angle, $\sin ^{2} \theta_{W}^{\text {eff }}$. Within the $S M$, this has a dramatic effect on the indirect determination of the Higgs-boson mass, providing a highly sensitive consistency test of the model that may possibly point towards large new-physics scales. Within the MSSM, the large increase in the precision of $\sin ^{2} \theta_{W}^{\text {eff }}$ will allow to obtain stringent indirect bounds on SUSY parameters. This will constitute, in analogy to the SM case, a powerful consistency test of supersymmetry at the quantum level and may be crucial to constrain SUSY parameters that are not directly experimentally accessible.

At the GigaZ option of the ILC a precision measurement of the effective leptonic weak mixing angle at the $Z$-boson resonance, $\sin ^{2} \theta_{W}^{\text {eff }}$, will be possible, allowing a very sensitive test of the electroweak theory [57]. The impact of the more precise measurement of $\sin ^{2} \theta_{W}^{\text {eff }}$ for testing the electroweak theory is illustrated in fig. 2.9 (left panel), where the experimental accuracy (using the current experimental central value of $\sin ^{2} \theta_{W}^{\text {eff }}[61]$ ) is compared with the predictions in the SM and the Minimal Supersymmetric extension of the Standard Model (MSSM), see also sect. 3.1. The theoretical predictions are shown for values of $m_{h}$ corresponding to the SM Higgs-boson mass and to the mass of the lightest CP-even Higgs boson $h$ in the MSSM, respectively. In the region where the two models overlap, $m_{h} \lesssim 140 \mathrm{GeV}[33,62]$, the SM prediction corresponds to the MSSM result in the limit where all SUSY partners are heavy. The area in the plot associated with the MSSM prediction was obtained by varying all relevant SUSY parameters independently, taking into account the constraints from the direct search for SUSY particles and the LEP Higgs search $[59,60]$. The MSSM predictions are based on the results described in [50], and the Higgs-mass predictions have been obtained with FeynHiggs2.0 [63]. For the top-quark mass a value of $m_{t}=175 \pm 0.1 \mathrm{GeV}$ has been used, assuming the prospective linear col- 


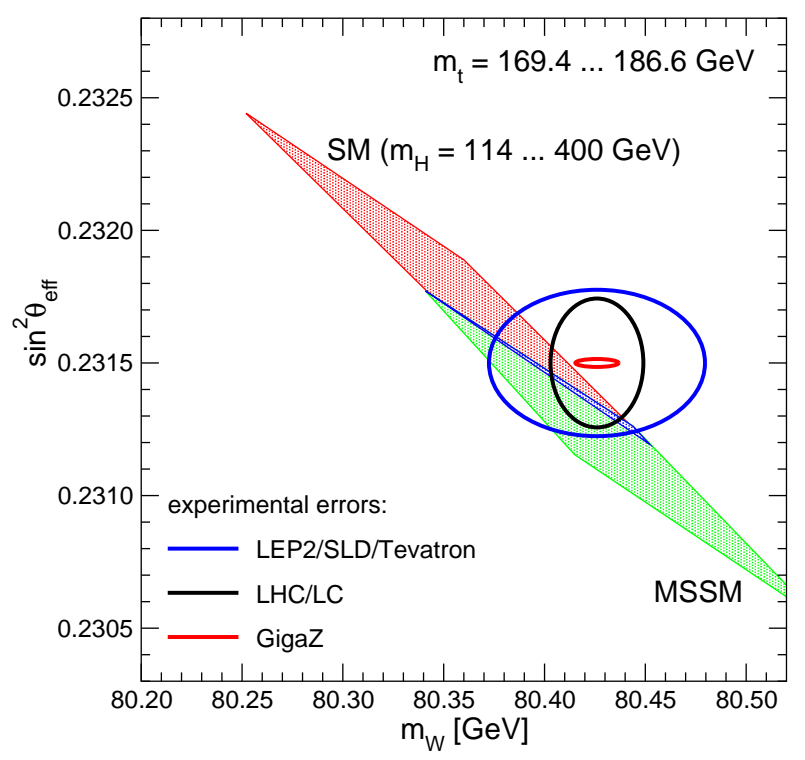

Figure 2.8: Prospective precision on $\sin ^{2} \theta_{W}^{\text {eff }}$ from $A_{\mathrm{LR}}$ : a high-precision measurement at GigaZ of the left-right asymmetry $A_{\mathrm{LR}}$ and consequently of $\sin ^{2} \theta_{\text {eff }}$ allows a test of the electroweak theory at an unprecedented level. The allowed parameter space of the SM and the MSSM in the $\sin ^{2} \theta_{\text {eff }}-m_{W}$ plane is shown together with the experimental accuracy reachable at GigaZ (applying the Blondel scheme) and polarized threshold scans for $m_{W}$. For comparison, the current experimental accuracy (LEP/SLD/Tevatron) and the prospective accuracy at the LHC and an LC without GigaZ option (LHC/LC) are also shown $[50,56,57]$.

lider accuracy (using instead a central value of $m_{t}=178 \mathrm{GeV}$ would induce a decrease in the $\sin ^{2} \theta_{W}^{\text {eff }}$ prediction of about $10^{-4}$ ).

The impact of the precision on $\sin ^{2} \theta_{W}^{\text {eff }}$ reachable with polarization of both beams compared to the case of electron polarization only can be clearly seen by confronting the SM prediction for $\sin ^{2} \theta_{W}^{\text {eff }}$ as function of $m_{h}$ with the prospective experimental accuracy. Requiring the SM prediction to agree with the $\sin ^{2} \theta_{W}^{\text {eff }}$ measurement at the one- $\sigma$ level (neglecting the uncertainties from unknown higher-order corrections) constrains the Higgsboson mass to an interval of few $\mathrm{GeV}$ for the case of simultaneous polarization of both beams, while a measurement of $\sin ^{2} \theta_{W}^{\text {eff }}$ based on electron polarization only leaves an uncertainty of about $\pm 25 \mathrm{GeV}$ in $m_{h}$.

Comparing the indirect constraints on the Higgs-boson mass with a direct measurement of $m_{h}$ provides a sensitive test of the electroweak theory at the quantum level. This is illustrated in fig. 2.9 (left panel), where a hypothetical measurement of $m_{h}=$ $115.5 \pm 0.5 \mathrm{GeV}$ is indicated. The assumed error of $0.5 \mathrm{GeV}$ summarizes both the prospective experimental error and the theoretical uncertainty in the relation between $m_{h}$ and $\sin ^{2} \theta_{W}^{\text {eff }}$. The latter is significantly larger in the MSSM, but for simplicity we use the more conservative MSSM value for both models. For the scenario shown in the plot the measurement of $\sin ^{2} \theta_{W}^{\text {eff }}$ based on electron polarization only combined with the $m_{h}$ measurement would not allow to distinguish between the predictions of the SM and the MSSM. 

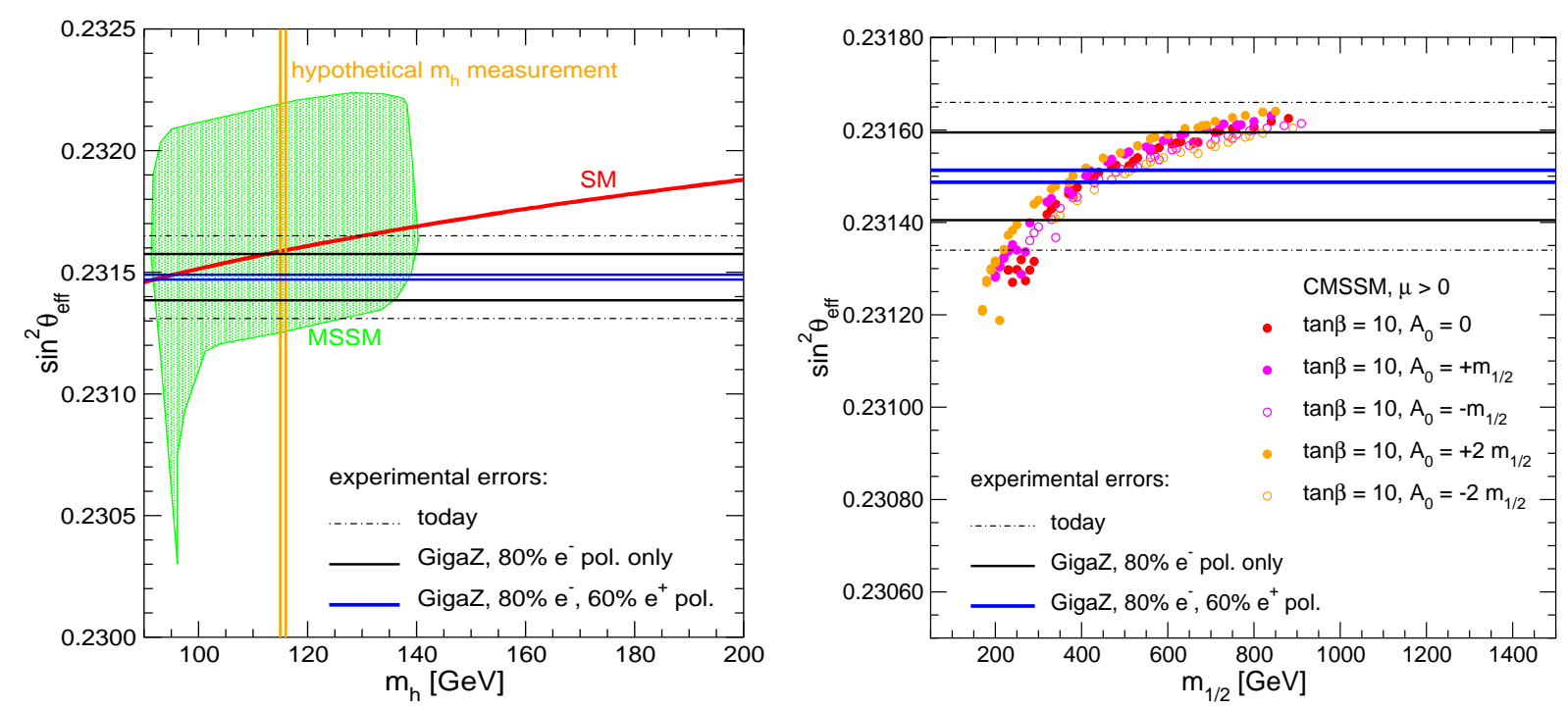

Figure 2.9: Left panel: the predictions for $\sin ^{2} \theta_{W}^{\text {eff }}$ in the SM and the MSSM are compared with the experimental accuracies obtainable at GigaZ with an $80 \%$ polarized electron beam only and with simultaneous polarization of both beams. The present experimental error on $\sin ^{2} \theta_{W}^{\text {eff }}$ is also indicated. The theoretical predictions are given in terms of $m_{h}$, which denotes the Higgs-boson mass in the SM and the mass of the lightest CP-even Higgs boson in the MSSM, respectively. The allowed area in the MSSM results from a scan over the relevant SUSY parameters entering via loop corrections. The sensitivity of the $\sin ^{2} \theta_{W}^{\text {eff }}$ measurement to deviations between the two models is illustrated for a hypothetical measurement of $m_{h}=115.5 \pm 0.5 \mathrm{GeV}$ [64]. Right panel: Allowed range for the SUSY mass parameter $m_{1 / 2}$ in a specific model, the CMSSM [64]. Experimental constraint from LEP searches as well as bounds from cold dark matter searches have been taken into account.

Both models would be compatible with the experimental measurements at the one- $\sigma$ level in this case. A measurement of $\sin ^{2} \theta_{W}^{\text {eff }}$ with simultaneous polarization of both beams, on the other hand, would resolve the different quantum corrections of the two models. The high-precision measurement of $\sin ^{2} \theta_{W}^{\text {eff }}$ combined with the $m_{h}$ measurement would show a large deviation from the SM prediction and would therefore indicate a clear preference for the MSSM. Within the MSSM the precision measurement provides a stringent consistency test, yielding sensitive constraints on the underlying SUSY parameters entering via quantum corrections.

In fig. 2.9 (right panel) the strong constraints on the parameter space of new physics models arising from the precision measurement of $\sin ^{2} \theta_{W}^{\text {eff }}$ are demonstrated [65]. Shown is the prediction of $\sin ^{2} \theta_{W}^{\text {eff }}$ as a function of $m_{1 / 2}$, the generic fermionic mass parameter in the CMSSM (the Constrained MSSM), see sect. 3.1. Experimental constraints from LEP searches as well as bounds from cold dark matter searches have been taken into account. The unprecedented accuracy in $\sin ^{2} \theta_{W}^{\text {eff }}$ strongly constrains the allowed parameter range for $m_{1 / 2}$, which is reduced by about a factor of 5 with $\left(\left|P_{e^{-}}\right|,\left|P_{e^{+}}\right|\right)=(80 \%, 60 \%)$ compared 
with the case of only polarized electrons $\left|P_{e^{-}}\right|=80 \%$.

Quantitative results: The gain of about one order of magnitude in the accuracy of the determination of $\sin ^{2} \theta_{W}^{\text {eff }}$ applying a Blondel scheme with $\left(\left|P_{e^{-}}\right|,\left|P_{e^{+}}\right|\right)=(80 \%, 60 \%)$ and $\Delta P_{e^{ \pm}} / P_{e^{ \pm}}=0.5 \%$ instead of using only $P_{e^{-}}=80 \%$, has strong impacts on bounds on the Higgs boson mass as well as on bounds on SUSY mass parameters. The resulting indirect bounds on the mass of the Higgs boson in the SM also improves by about an order of magnitude. Regarding a specific SUSY model the allowed parameter range for the specific SUSY mass parameters $m_{1 / 2}$ is reduced by about a factor five.

\subsubsection{CP violation in 3-jet and 4-jet decays of the Z-boson at GigaZ}

An interesting sector where to look for physics beyond the SM is represented by CPviolating $Z$ decays to heavy leptons and quarks, in particular to final states with $b \bar{b}$ pairs. The high statistics available at GigaZ should allow a search for signals from nonstandard CP-violating couplings with unprecedented sensitivity. It is also improved by initial electron and positron longitudinal polarization. It is expected that one can reach an accuracy of about an order of magnitude better than at LEP.

$\mathrm{CP}$-violating effects in flavour-diagonal $Z$ decays are found to be extremely small in the SM [66], therefore this sector seems particularly suitable for non-standard physics searches. A relevant example is represented by the processes $Z \rightarrow b \bar{b} g$ and $Z \rightarrow b \bar{b} g g$, where $g$ denotes the gluon, leading to 3-jet and 4-jet final states, respectively $[67,68]$. The $\mathrm{CP}$-violating couplings in the $Z b \bar{b} g$ vertex and in the $Z b \bar{b} g g$ vertex (the latter related to the former by QCD gauge invariance), have been searched for at LEP, where no significant deviation from the SM was found [70].

For a model-independent analysis of CP-violation in the above processes, an effective Lagrangian approach is most convenient [66] and, accordingly, one can add to the SM the $\mathrm{CP}$-violating $Z \bar{b} \bar{b} g$ four-point vertex:

$$
\mathcal{L}_{\mathrm{CP}}(x)=\left[h_{V b} \bar{b}(x) T^{a} \gamma^{\nu} b(x)+h_{A b} \bar{b}(x) T^{a} \gamma^{\nu} \gamma_{5} b(x)\right] Z^{\mu}(x) G_{\mu \nu}^{a}(x),
$$

where $T^{a}$ are the familiar $\mathrm{SU}(3)_{\mathrm{C}}$ generators. Here, $h_{V b}$ and $h_{A b}$ are real CP-violating vector and axial-vector chirality-conserving coupling constants. Due to the quadratic gluon term present in $G_{\mu \nu}^{a}$, the vertex eq. (2.14) is involved in both $Z \rightarrow 3$ jets and in $Z \rightarrow 4$ jets. Dimensionless coupling constants $\widehat{h}_{V b, A b}$ can then be defined by using $m_{Z}$ as a scale parameter, through the relations $h_{V b, A b}=e g_{s} \widehat{h}_{V b, A b} /\left(\sin \theta_{W} \cos \theta_{W} m_{Z}^{2}\right)$, with $g_{s}$ the QCD coupling constant.

Such chirality-conserving CP-violating interactions (2.14) can originate in different scenarios, for instance in multi-Higgs extensions of the SM [71] and in models with excited quarks, typical of compositeness, where quarks have substructure [72].

In the latter scenario, one assumes that the $b$ quark has an excited partner $b^{\prime}$ with mass $m_{b^{\prime}}$ and spin $\frac{1}{2}$. Both chirality-conserving $Z b^{\prime} b$ couplings at the GigaZ scale and chirality-flipping $b b^{\prime} g$ couplings, allowed in such composite models, can be expressed via an effective interaction of the form

$$
\begin{aligned}
\mathcal{L}^{\prime}(x)= & -\frac{e}{2 \sin \theta_{W} \cos \theta_{W}} Z_{\mu}(x) \bar{b}^{\prime}(x) \gamma^{\mu}\left(g_{V}^{\prime}-g_{A}^{\prime} \gamma_{5}\right) b(x) \\
& -i \frac{g_{s}}{2 m_{b^{\prime}}} \hat{d}_{c} \bar{b}^{\prime}(x) \sigma^{\mu \nu} \gamma_{5} T^{a} b(x) G_{\mu \nu}^{a}(x)+\text { h.c. }
\end{aligned}
$$


Here $g_{V}^{\prime}, g_{A}^{\prime}$ and $\hat{d}_{c}$ are complex constants that, as is conventional for composite models, can be expected to be of order unity if the novel dynamics underlying compositeness is strong. It is understood that $m_{b^{\prime}} \gg m_{Z}$, and from virtual $b^{\prime}$ exchange one derives for the couplings in eq. (2.14): $\widehat{h}_{V b}=\operatorname{Re}\left(\hat{d}_{c} g_{A}^{\prime *}\right) m_{Z}^{2} / m_{b^{\prime}}^{2}$ and $\widehat{h}_{A b}=-\operatorname{Re}\left(\hat{d}_{c} g_{V}^{\prime *}\right) m_{Z}^{2} / m_{b^{\prime}}^{2}$ [71].

Assuming flavour-tagging [1], the $b$-quark momenta can be reconstructed. CP-violating couplings may then be searched for by using CP-odd observables constructed from the $b$ and $\bar{b}$ quark momenta $[66,67]$ (with ' 3 ' the cartesian component in the $Z$ rest frame corresponding to the positron beam):

$$
\begin{gathered}
T_{33}=\left(\widehat{\mathbf{k}}_{\bar{b}}-\widehat{\mathbf{k}}_{b}\right)_{3}\left(\widehat{\mathbf{k}}_{\bar{b}} \times \widehat{\mathbf{k}}_{b}\right)_{3}, \\
V_{3}=\left(\widehat{\mathbf{k}}_{\bar{b}} \times \widehat{\mathbf{k}}_{b}\right)_{3} .
\end{gathered}
$$

The observable $T_{33}$ transforms as a tensor component, $V_{3}$ as a vector component.

To a very good approximation, for $Z \rightarrow 3$ jets and $Z \rightarrow 4$ jets the tensor observable $T_{33}$ and the vector one $V_{3}$ turn out to be sensitive only to the combinations $\widehat{h}_{b}=\widehat{h}_{A b} g_{V b}-\widehat{h}_{V b} g_{A b}$ and $\widetilde{h}_{b}=\widehat{h}_{V b} g_{V b}-\widehat{h}_{A b} g_{A b}$, respectively, where $g_{V b}=-\frac{1}{2}+\frac{2}{3} \sin ^{2} \theta_{W}$ and $g_{A b}=-\frac{1}{2}$ [67]. Clearly, a non-zero value of one of the above observables is an unambiguous indicator of CP violation. Note that for longitudinally-polarized beams, the additional assumption that the $Z e^{+} e^{-}$vertex is free from chirality-flipping interactions is necessary.

\section{Numerical results}

The sensitivities of the observables (2.16) and (2.17) on the couplings $\widehat{h}_{b}$ and $\widetilde{h}_{b}$ depend on the jet resolution cut $y_{\text {cut }}$, see also [73]. The expectation values of $T_{33}$ do not depend on initial longitudinal polarization, and therefore the sensitivity to $\widehat{h}_{b}$ only reflects the statistics.

The one- $\sigma$ uncertainty on the measurement of $\widetilde{h}_{b}$ through the observable (2.17) is evaluated on a statistical basis of $N=10^{9} Z$ decays with unpolarized beams. The results for $Z \rightarrow 3$ jets and different choices of beam polarizations are shown in table 2.10 (the results for $Z \rightarrow 4$ jets are presented in [68]). As indicated by the table, the advantage of initial beam polarization is significant: for $P_{e^{-}}=-80 \%$ and $P_{e^{+}}=+60 \%$ the sensitivity is maximal, more than a factor of 6 compared to the case of unpolarized beams (the improvement due to additional positron polarization leads to a factor of about 1.5 compared to the case where only electrons are polarized). On the other hand, numerical results show a stability against changes in the jet resolution parameter $y_{\text {cut }}$ up to 0.1 . Notice also, that the results in table 2.10 are obtained by assuming, for the measurement of the vector observable $V_{3}$, a $100 \% b-\bar{b}$ distinction. In the case of no experimental signal at one- $\sigma$, lower bounds on $m_{b^{\prime}}$ can be derived. These are also shown in table 2.10 under the assumption $\operatorname{Re}\left(\hat{d}_{c} g_{A}^{\prime *}\right)=\operatorname{Re}\left(\hat{d}_{c} g_{V}^{\prime *}\right)=1$.

As can be seen, the coupling $\widetilde{h}_{b}$ could be measured at GigaZ with an accuracy of order $1.5 \times 10^{-3}$ (one- $\sigma$ level) from $Z \rightarrow 3$ jets (one obtains qualitatively similar results from $Z \rightarrow 4$ jets for lower values of $\left.y_{\text {cut }}\right)$. In the case of non-observation of the effect at the one- $\sigma$ level, the lower bound on the $m_{b^{\prime}}$ mass, $m_{b^{\prime}}>2.2 \mathrm{TeV}$, can be derived, assuming as usual the couplings of the novel compositeness interaction to be of the strong interaction size. Such bound should be compared with the best current limits $m_{q^{\prime}}>775 \mathrm{GeV}$ [74] that, 
however, applies to $u$ - and $d$-quarks but may not exclude lighter $b^{\prime}$ excitations. ${ }^{\ddagger}$ Although not sensitive to beam polarization, i.e. unpolarized beams would be sufficient, one may mention for the case of the observable $T_{33}$ that the one- $\sigma$ accuracy on $\widehat{h}_{b}$ would be of order 0.004 from $Z \rightarrow 3$ jets.

In a realistic analysis, also actual reconstruction efficiences and systematic uncertainties should be taken into account. Nevertheless, assuming them to be of the same size as the statistical uncertainties, one could conclude that the achievable sensitivities to $\widehat{h}_{b}$ and $\widetilde{h}_{b}$ are one order of magnitude better than those obtained at LEP. This can give valuable information on, e.g., the scalar sector in multi-Higgs extensions of the SM, as well as provide stringent tests of models with excited quarks.

\begin{tabular}{|c|c|c|c|c|c|c|}
\hline & \multicolumn{5}{|c|}{$\left(P_{e^{-}}, P_{e^{+}}\right)$} \\
$V_{3}$ & $y_{\text {cut }}$ & $(0,0)$ & $(+80 \%, 0)$ & $(-80 \%, 0)$ & $(+80 \%,-60 \%)$ & $(-80 \%,+60 \%)$ \\
\hline$\Delta \widetilde{h}_{b}\left[10^{-4}\right]$ & 0.01 & 85 & 27 & 19 & 18 & 14 \\
& 0.1 & 94 & 30 & 21 & 20 & 15 \\
\hline$m_{b^{\prime}}[\mathrm{TeV}]$ & 0.01 & 0.91 & 1.6 & 1.9 & 2.0 & 2.3 \\
& 0.1 & 0.87 & 1.5 & 1.8 & 1.9 & 2.1 \\
\hline
\end{tabular}

Table 2.10: Accuracy on the vector observable $V_{3}$ on $\Delta \widetilde{h}_{b}$ and corresponding lower limits on the excited quark mass $m_{b^{\prime}}$ at the one- $\sigma$ level. [69].

Quantitative example: Having both beams polarized with $\left|P_{e^{-}}\right|=80 \%,\left|P_{e^{+}}\right|=$ $60 \%$ improves the sensitivity by up to a factor 6 compared with using unpolarized beams and by about 20-35\% compared with the case when only electrons are polarized.

\footnotetext{
${ }^{\ddagger}$ These numbers should be compared to the excited quark mass limits at the 2- $\sigma$ level. In that case a measurement of $\widehat{h}_{b} \widetilde{h}_{b}$ has to produce a mean value larger than $2 \Delta \widehat{h}_{b}, 2 \Delta \widetilde{h}_{b}$ to be able to claim a non-zero effect. The mass limits at the one- $\sigma$ level given in table 2.10 have to be divided by a factor $\sqrt{2}$ to get the limits at the 2- $\sigma$ level.
} 


\section{Chapter 3}

\section{Searches for new physics with polarized $e^{-}$and $e^{+}$beams}

\subsection{Supersymmetry}

\subsubsection{Introduction and choice of SUSY scenarios}

The importance of the polarization of both beams at the ILC is here demonstrated for supersymmetry, which is one of the best motivated possibilities for New Physics (NP). With supersymmetry the unification of the $\mathrm{U}(1), \mathrm{SU}(2)$ and $\mathrm{SU}(3)$ gauge couplings is possible and this new symmetry also stabilizes the Higgs mass with respect to radiative corrections. If nature is supersymmetric at the electroweak scale, there is a priori a large number of parameters specifying different scenarios.

The Minimal Supersymmetric Standard Model (MSSM) is the minimal extension of the SM particle sector to incorporate supersymmetry. In addition to the particles of the $\mathrm{SM}$, the MSSM contains their supersymmetric partners: sleptons $\tilde{\ell}^{ \pm}, \tilde{\nu}_{\ell}(\ell=e, \mu, \tau)$, squarks $\tilde{q}$, charginos $\tilde{\chi}_{1,2}^{ \pm}$and neutralinos $\tilde{\chi}_{i}^{0}, i=1, \ldots, 4$. Two complex Higgs doublets $\left(H_{1}^{0}, H^{+}\right),\left(H^{-}, H_{2}^{0}\right)$ are needed to generate the masses of up- and down-quarks. They lead to five physical Higgs bosons, the neutral $h, H$ (CP-even), $A$ (CP-odd) and the charged $H^{ \pm}$particles.

Both the SM and the SUSY partners are described in common multiplets and carry the same quantum numbers-with the exception of the spin quantum number, which differs by half a unit. Since the SUSY partners are not degenerate in mass with their SM partners, SUSY has to be a broken symmetry. In a most general parametrization, soft supersymmetry-breaking terms with many new parameters are introduced. Due to the electroweak symmetry breaking and the SUSY breaking, the interaction eigenstates of the fermions, gauginos and higgsinos $\tilde{W}^{ \pm}, \tilde{H}_{1,2}^{ \pm}\left(\tilde{\gamma}, \tilde{Z}, \tilde{H}_{1,2}^{0}\right)$, i.e. the SUSY partners of the charged (neutral) gauge and Higgs bosons, mix to form mass eigenstates $\tilde{\chi}_{1,2}^{ \pm}\left(\tilde{\chi}_{1, \ldots, 4}^{0}\right)$. The masses and couplings of the charginos and neutralinos are determined by the corresponding mass matrices, which depend on the $\mathrm{U}(1)(\mathrm{SU}(2))$ gaugino mass parameters $M_{1}\left(M_{2}\right)$, the higgsino mass parameter $\mu$ and the ratio of the Higgs expectation values $\tan \beta=v_{2} / v_{1}$, see e.g. [75].

Corresponding to the two chirality states of the leptons and quarks one has the left and right scalar partners $\tilde{\ell}_{L}, \tilde{q}_{L}$ and $\tilde{\ell}_{R}, \tilde{q}_{R}$. The mass matrices of the sfermions depend on scalar mass parameters $M_{Q}, M_{U}, M_{D}, M_{L}, M_{E}$ trilinear couplings $A_{\ell}, A_{q}$ and $\mu$ and $\tan \beta$. 
Mixing effects between the R- and L-scalar states are expected to be most important for the third generation; the corresponding mass eigenstates are called $\tilde{t}_{1}, \tilde{b}_{1}, \tilde{\tau}_{1}$ and $\tilde{t}_{2}, \tilde{b}_{2}, \tilde{\tau}_{2}$.

In order to distinguish between SUSY and SM particles a new quantum number Rparity is introduced, $\mathrm{R}=-1$ for SUSY particles, and +1 for SM particles. If R-parity is conserved, the SUSY particles can only be produced in pairs and the lightest SUSY particle has to be stable and represents the final particle of all decay chains of SUSY particles. In all studies shown here, the lightest SUSY particle is assumed to be the lightest neutralino $\tilde{\chi}_{1}^{0}$, which is also a good candidate for the cold dark matter particle.

Since in SUSY also many new sources of CP violation could occur, one ends up, even in the MSSM with conserved R-parity, with 105 new parameters. With specific model assumptions about the SUSY breaking mechanism and mass unifications, the number of free parameters is strongly reduced. In the so-called minimal supergravity (mSUGRA) model, for example, one has only three parameters and one sign. However, one should keep in mind, that at future experiments at the LHC and the LC, one has-after detecting signals expected by SUSY - to determine the parameters as model-independently as possible and to confirm the underlying assumptions.

\section{Choice of SUSY scenarios}

A priori it can not be stated whether one specific point in the multi-parameter space of SUSY is less probable than others. There exist mass limits from the direct searches for Higgs and SUSY particles at LEP and the Tevatron and indirect bounds from tests of electroweak precision observables and of searches for $g_{\mu}-2$ [76] and $b \rightarrow s \gamma$ [78] from low-energy experiments. Indirect limits for the CP-violating phases can be derived from the EDM of the electron, neutron and atomic systems [79]. Further indirect bounds for the MSSM parameter space can be derived from dark matter searches [80]. The chosen point has to be checked case-by-case whether it violates any experimental bounds. If a specific SUSY breaking scheme has been assumed, as e.g. mSUGRA, exclusion bounds in the corresponding parameter space can be derived due to the restricted small number of parameters. However, such choices reduce the variety of possible signatures considerably.

In order to give an overview of the many effects of beam polarization in searches for supersymmetry the chosen SUSY scenarios have not been restricted to a specific SUSY breaking scheme and unification assumptions. This approach has led to the consideration, in this report, of a large variety of different SUSY scenarios. In some examples polarizing both beams is absolutely necessary for analyzing the properties of particles and couplings. In other studies the polarization of both beams is needed for quantitative reasons and leads to better statistics, i.e., higher cross sections and better background suppression, which can be decisive for a discovery. Therefore, having both beams polarized is crucial to face the many challenges in supersymmetry and to resolve and determine precisely this candidate for a new theory.

Table 3.1 provides an overview of different parameters sets, together with references to the topic that is addressed in the study.

\subsubsection{Determination of selectron properties}

In order to test whether the SUSY partners of the electrons/positrons carry the same 


\begin{tabular}{|c|c|c|c|c|c|c|c|c|}
\hline & $\left|M_{1}\right|$ & $\varphi_{M_{1}}$ & $M_{2}$ & $|\mu|$ & $\varphi_{\mu}$ & $\tan \beta$ & scalar sector parameters & topic of study \\
\hline \multicolumn{8}{|c|}{ MSSM scenarios without CP-violating phases: } & \multirow{5}{*}{$\begin{array}{l}\text { selectron properties } \\
\text { selectron proporties } \\
\text { smuon masses } \\
\text { stau properties }\end{array}$} \\
\hline S1 & 150 & 0 & 210 & 400 & 0 & 20 & $m_{\tilde{e}_{R, L}}=195,200 \mathrm{GeV}$ & \\
\hline S2 & 103 & 0 & 232 & 403 & 0 & 10 & $m_{\tilde{e}_{R, L}}=187,223 \mathrm{GeV}$ & \\
\hline $\mathrm{S}^{*}$ & 163 & 0 & 311 & 509 & 0 & 10 & $m_{\tilde{\mu}_{R, L}}=178,287 \mathrm{GeV}$ & \\
\hline S4 & 99 & 0 & 193 & 140 & 0 & 20 & $\begin{array}{l}m_{\tilde{\tau}_{1}}=155 \mathrm{GeV} \\
\left|\cos \theta_{\tilde{\tau}}\right|=0.08\end{array}$ & \\
\hline S5 & - & - & - & - & - & - & $\begin{array}{l}m_{\tilde{t}_{1}}=200 \mathrm{GeV} \\
\left|\cos \theta_{\tilde{t}}\right|=0.4 \text { and } 0.66\end{array}$ & stop properties \\
\hline$S 6^{\dagger}$ & GUT & 0 & 193 & 352 & 0 & 10 & $m_{\nu_{e}, \tilde{e}_{L}}=186,202 \mathrm{GeV}$ & charginos \\
\hline S7 & 90 & 0 & 350 & 140 & 0 & 20 & $m_{\tilde{e}_{L, R}}$ variable & neutralinos \\
\hline S15 & 100 & 0 & 200 & 1000 & 0 & {$[2,30]$} & $\begin{array}{l}M_{\tilde{Q}}=M_{\tilde{U}}=350, \\
A_{t}=A_{b}=700+\mu / \tan \beta \\
m_{A}=[400,800] \mathrm{GeV}\end{array}$ & heavy Higgs \\
\hline \multicolumn{8}{|c|}{ MSSM scenarios with CP-violating phases: } & CP asymmetries: \\
\hline S8 & 200 & $\pi / 5$ & 400 & 240 & 0 & 10 & $m_{\tilde{e}_{R, L}}=220,372 \mathrm{GeV}$ & $\tilde{\chi}_{2}^{0}:$ 2-body decay \\
\hline S9 & 100 & 0 & 200 & 250 & 0 & 5 & $\begin{array}{l}m_{\tilde{\tau}_{1,2}}=143,210 \mathrm{GeV} \\
\left|A_{\tau}\right|=1500, \varphi_{A_{\tau}}=\pi / 2\end{array}$ & $\begin{array}{l}\tilde{\chi}_{2}^{0}: \text { 2-body decay } \\
\quad \text { into } \tau ; \varphi_{A_{\tau}} \neq 0\end{array}$ \\
\hline S10 & 100 & $\pi / 5$ & 200 & 250 & 0 & 5 & $\begin{array}{l}m_{\tilde{\tau}_{1,2}}=144,209 \mathrm{GeV} \\
A_{\tau}=250, \varphi_{A_{\tau}}=0\end{array}$ & $\begin{array}{l}\tilde{\chi}_{2}^{0}: \text { 2-body decay } \\
\quad \text { into } \tau ; \varphi_{M_{1}} \neq 0\end{array}$ \\
\hline S11 & 150 & $\pi / 5$ & 300 & 200 & 0 & 10 & $m_{\tilde{e}_{R, L}}=224,268 \mathrm{GeV}$ & $\tilde{\chi}_{2}^{0}:$ 3-body decay \\
\hline S12 & GUT & $\pi / 2$ & $M_{2}-|\mu|$ & variable & 0 & 3 & $m_{\tilde{e}_{R, L}}=150,400 \mathrm{GeV}$ & $\tilde{\chi}_{1}^{0} \tilde{\chi}_{2}^{0}$ with $P_{e^{-}}^{\mathrm{T}}, P_{e^{+}}^{\mathrm{T}}$ \\
\hline \multicolumn{8}{|c|}{ Scenarios in extended SUSY models: } & \\
\hline S13 a) & 195 & 0 & 300 & 350 & 0 & 20 & $m_{\tilde{e}_{R, L}}=143,202 \mathrm{GeV}$ & distinction: \\
\hline S13 b) & 270 & 0 & 381 & - & 0 & 20 & $\begin{array}{l}m_{\tilde{e}_{R, L}}=143,202 \mathrm{GeV} \\
\mu_{\mathrm{eff}}=350, \kappa=0.152\end{array}$ & $\mathrm{MSSM} \leftrightarrow \mathrm{NMSSM}$ \\
\hline S14 & - & - & - & - & - & - & $\begin{array}{l}m_{\tilde{\nu}_{e}}=650, \Gamma_{\tilde{\nu}_{e}}=1 \\
\lambda_{131}=0.05\end{array}$ & $\begin{array}{l}\text { R-parity violation: } \\
\text { spin-0 in s-channel }\end{array}$ \\
\hline
\end{tabular}

Table 3.1: SUSY parameters of the scenarios studied in this report: the U(1) (SU(2)) gaugino mass parameters $M_{1}\left(M_{2}\right)$, the higgsino mass parameter $\mu$, the ratio of the Higgs vacuum expectation values $\tan \beta=v_{2} / v_{1}$, the mass of the CP-odd Higgs $m_{A}$, the slepton masses $m_{\tilde{\ell}_{L, R^{\prime}}}$ the trilinear couplings of the $3 \mathrm{rd}$ generations $A_{t}, A_{b}, A_{\tau}$ and the possible non-vanishing phases $\varphi_{M_{1}}, \varphi_{\mu}, \varphi_{A_{t, b, \tau}}$. All mass parameters and trilinear couplings are given in $\mathrm{GeV}$. In case that unification between the $\mathrm{U}(1)$ and $\mathrm{SU}(2)$ parameters is assumed, the absolute value of $M_{1}$ is given by $\left|M_{1}\right|=\frac{5}{3} \tan ^{2} \theta_{W} M_{2}$ and is denoted by 'GUT'. Listed are only those parameters which are relevant for the presented study. The large number of scenarios reflects the variety of possible different signatures in the MSSM; the scenario number reflects sequence of corresponding plots in this chapter $\left({ }^{*} \equiv \mathrm{SPS} 3\right.$ and $\dagger \equiv$ SPS1a [81]). 
chiral quantum numbers as their SM partners one has to separate the scattering process from the annihilation process. With both beams polarized the production vertices in the $t$ - and $u$-channel can be analysed independently. Another important test of the theory is to show that the SUSY Yukawa couplings are equal to the gauge couplings. Polarized positrons are needed for such model tests, in particular in scenarios where even a fully polarized electron beam is insufficient.

In this section selectron production, $e^{+} e^{-} \rightarrow \tilde{e}_{L, R}^{+} \tilde{e}_{L, R}^{-}$, with polarized beams is studied. The process occurs via $\gamma, Z$ exchange in the $s$-channel and via neutralino exchanges, $\tilde{\chi}_{1,2,3,4}^{0}$, in the $t$-channel, see fig. 3.1. In the $t$-channel both pair production, $\tilde{e}_{L}^{+} \tilde{e}_{L}^{-}, \tilde{e}_{R}^{+} \tilde{e}_{R}^{-}$, as well as associated production, $\tilde{e}_{L}^{+} \tilde{e}_{R}^{-}, \tilde{e}_{R}^{+} \tilde{e}_{L}^{-}$, is possible, whereas in the $s$-channel only pairs, $\tilde{e}_{L}^{+} \tilde{e}_{L}^{-}, \tilde{e}_{R}^{+} \tilde{e}_{R}^{-}$, can be produced. In the MSSM at tree-level this sector depends on the scalar masses and, due to the exchange of all neutralinos in the $t$-channel, on the gaugino/higgsino mixing parameters $M_{1,2}, \varphi_{M_{1}}, \mu, \varphi_{\mu}$ and $\tan \beta$.

In the following the impact of beam polarization for determining a) the quantum numbers $L, R$ and $\mathrm{b}$ ) the Yukawa couplings is studied.
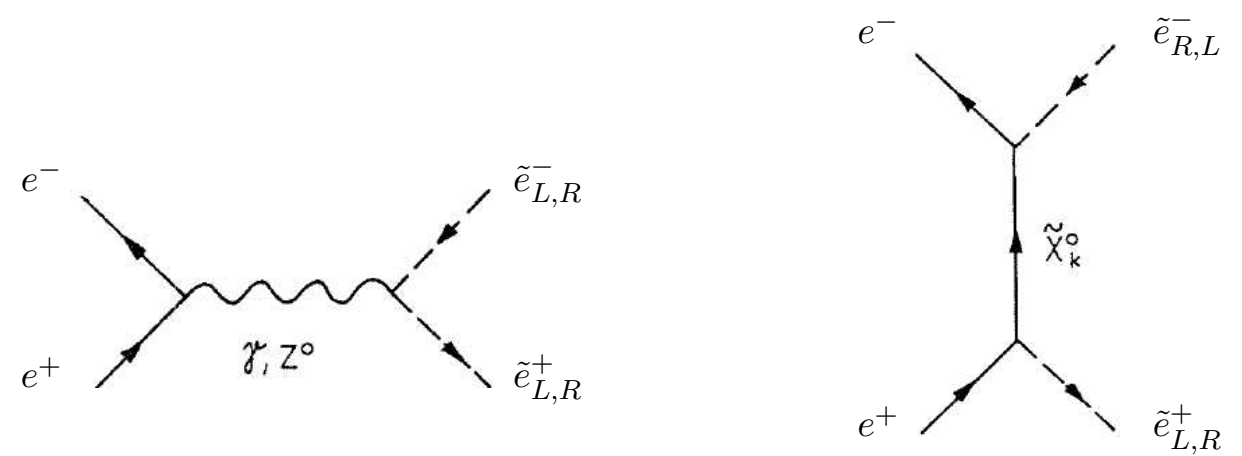

Figure 3.1: Selectron production: $\gamma, Z$-exchange in the $s$-channel and $\tilde{\chi}_{1}^{0}, \ldots, \tilde{\chi}_{4}^{0}$-exchange in the $t$-channel.

\section{Chiral quantum numbers}

Supersymmetry associates scalars to chiral (anti)fermions

$$
e_{\mathrm{L}, \mathrm{R}}^{-} \leftrightarrow \tilde{e}_{\mathrm{L}, \mathrm{R}}^{-} \quad \text { and } \quad e_{\mathrm{L}, \mathrm{R}}^{+} \leftrightarrow \tilde{e}_{\mathrm{R}, \mathrm{L}}^{+}
$$

In order to prove this association it is necessary to have both beams polarized [82]. The association can be directly tested only in the $t$-channel, as can be inferred from fig. 3.1. Polarized beams serve to separate this channel from the $s$-channel and enhance the cross section of just those SUSY partners of the initial chiral $e_{\mathrm{L}, \mathrm{R}}^{-}$and $e_{\mathrm{L}, \mathrm{R}}^{+}$given by the beam polarization, see eq. (3.1). This is demonstrated by isolation of $\tilde{e}_{\mathrm{L}}^{+} \tilde{e}_{\mathrm{R}}^{-}$by the RR configuration of the initial beams in an example where the selectron masses are close together, namely $m_{\tilde{e}_{\mathrm{L}}}=200 \mathrm{GeV}, m_{\tilde{e}_{\mathrm{R}}}=195 \mathrm{GeV}$ so that both $\tilde{e}_{\mathrm{L}}, \tilde{e}_{\mathrm{R}}$ decay via the same channels, $\tilde{e}_{\mathrm{L}, \mathrm{R}} \rightarrow \tilde{\chi}_{1}^{0} e$. The decay products can be separated e.g. via their different energy spectra and charge separation. At the LC it is then possible to measure the selectron masses with an expected accuracy of typically a few hundred MeV [1]. In addition, all SM background events, e.g., those from $W^{+} W^{-}$production, are strongly suppressed with the RR configuration. The other SUSY parameters correspond to the scenario S1 in table 3.1. 
The importance of having both beams polarized is demonstrated in fig. 3.2, which exhibits the isolation of the $\tilde{e}_{\mathrm{L}}^{+} \tilde{e}_{\mathrm{R}}^{-}$pair. Even extremely high right-handed electron polarization, $P_{e^{-}} \geq+90 \%$, is not sufficient by itself to disentangle the pairs $\tilde{e}_{\mathrm{L}}^{+} \tilde{e}_{\mathrm{R}}^{-}$and $\tilde{e}_{\mathrm{R}}^{+} \tilde{e}_{\mathrm{R}}^{-}$and to test their association to the chiral quantum numbers, since both cross sections are numerically very close, as seen in fig. 3.2 (left panel). Only with right-handed polarizations of both beams, the pair $\tilde{e}_{\mathrm{L}}^{+} \tilde{e}_{\mathrm{R}}^{-}$can be separated, as seen in fig. 3.2 (right panel).

Note that the $t$ - ( $s$-wave) and $s$-channel ( $p$-wave) production could also be separated via threshold scans [83], where sufficient running time at different energies close to the threshold is required. It is, however, also necessary to have both beams polarized in that case to test whether indeed the couplings of the produced selectrons uniquely correspond to the chirality of the electrons/positrons, respectively, as in eq. (3.1).
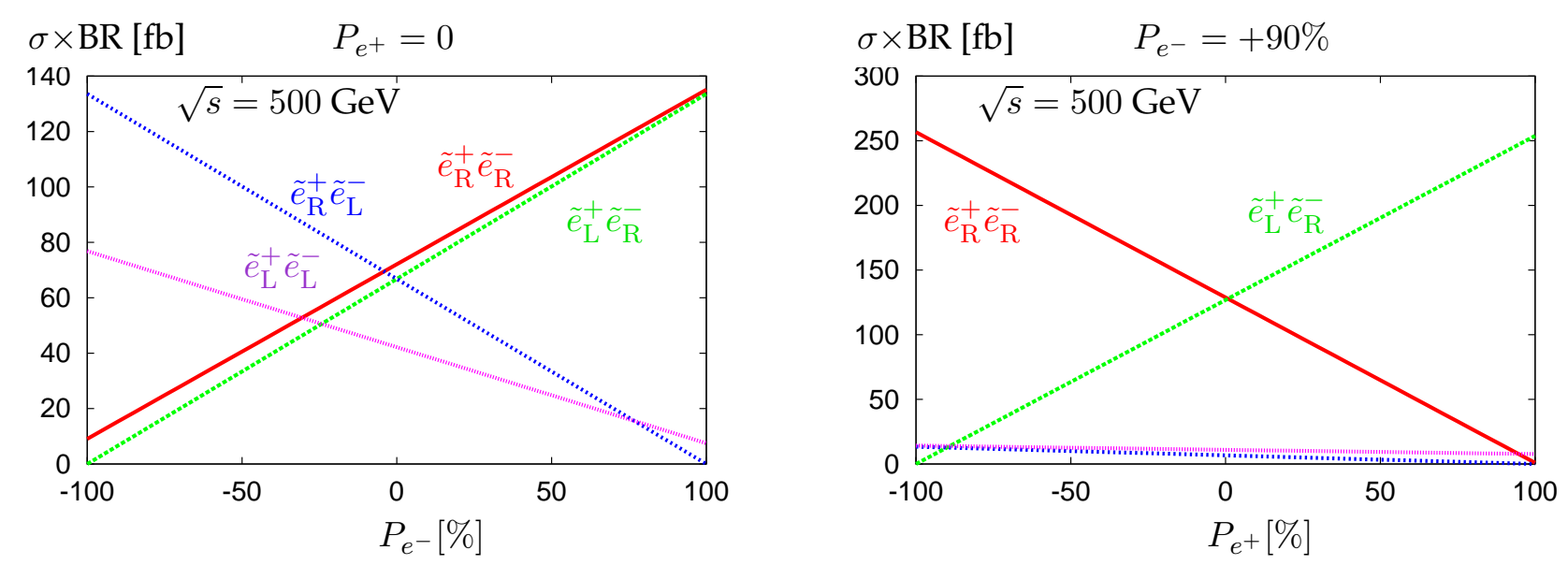

Figure 3.2: Separation of the selectron pair $\tilde{e}_{\mathrm{L}}^{+} \tilde{e}_{\mathrm{R}}^{-}$in $e^{+} e^{-} \rightarrow \tilde{e}_{\mathrm{L}, \mathrm{R}}^{+} \tilde{e}_{\mathrm{L}, \mathrm{R}}^{-} \rightarrow e^{+} e^{-} 2 \tilde{\chi}_{1}^{0}$ is not possible with electron polarization only (left panel). If, however, both beams are polarized, the cross sections (right panel) differ and the RR configuration separates the pair $\tilde{e}_{\mathrm{L}}^{+} \tilde{e}_{\mathrm{R}}^{-}$[84]. The SUSY parameters are chosen as in scenario S1, table 3.1.

\section{Yukawa couplings}

As a consequence of supersymmetry, the SU(2) and U(1) SUSY Yukawa couplings have to be identical to the corresponding SM gauge couplings. Assuming that the masses and mixing parameters of the neutralinos are known, the production cross sections of $\tilde{e}_{\mathrm{R}}^{+} \tilde{e}_{\mathrm{R}}^{-}$ and $\tilde{e}_{\mathrm{L}}^{+} \tilde{e}_{\mathrm{R}}^{-}$can be exploited to derive the Yukawa couplings. In [85] a one- $\sigma$ uncertainty of $0.2 \%(1.2 \%)$ in the determination of the $\mathrm{U}(1)(\mathrm{SU}(2))$ Yukawa couplings has been derived for the SUSY reference scenario SPS1a [81]. The study was done at $\sqrt{s}=500 \mathrm{GeV}$, $\mathcal{L}_{\text {int }}=500 \mathrm{fb}^{-1}$, including specific cuts to reduce the SM background and taking also into account effects from beamstrahlung and initial-state radiation (ISR). With $\left(\left|P_{e^{-}}\right|,\left|P_{e^{+}}\right|\right)=$ $(80 \%, 50 \%)$ the result is improved by a factor of 1.4 compared with the case of $(80 \%, 0)$.

In this analysis performed in the SPS1a scenario, the chirality of the produced selectrons can be distinguished by their decay modes, since L-selectrons can decay into the second-lightest neutralino $\tilde{\chi}_{2}^{0}$, while for the R-selectrons only the decay channel $\tilde{e}_{\mathrm{R}}^{ \pm} \rightarrow$ $e^{ \pm} \tilde{\chi}_{1}^{0}$ is open. For a slightly heavier gaugino mass $M_{1 / 2}$ and smaller scalar mass $m_{0}$, however, both selectron states have identical decay modes, $\tilde{e}_{\mathrm{R}, \mathrm{L}}^{ \pm} \rightarrow e^{ \pm} \tilde{\chi}_{1}^{0}$. In this case the 


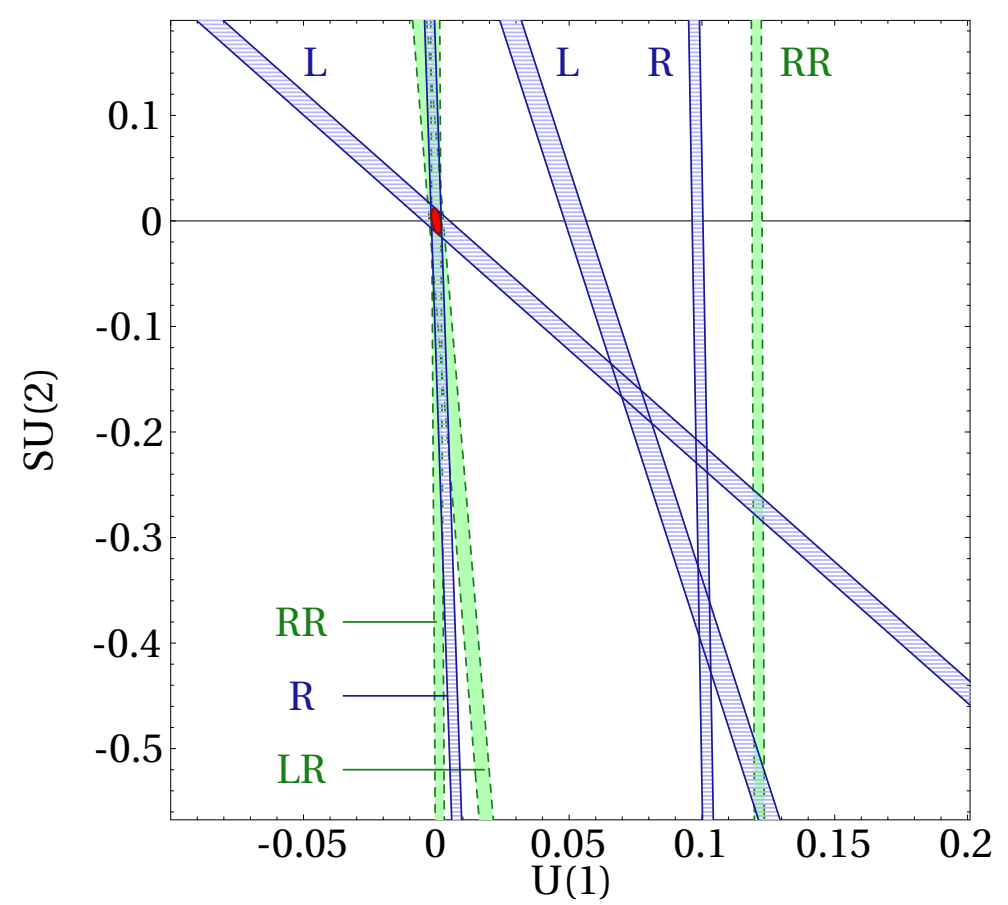

Figure 3.3: $1 \sigma$ bounds on the determination of the supersymmetric $\mathrm{U}(1)$ and $\mathrm{SU}(2)$ Yukawa couplings between $e^{+}, \tilde{e}_{\mathrm{R}, \mathrm{L}}^{+}$and $\tilde{\chi}_{i}^{0}$ from selectron cross-section measurements. The blue (shaded) bands indicate results from measurements using only electron beam polarization for two values $P_{e^{-}}=+90 \%(\mathrm{R})$ and $P_{e^{-}}=-90 \%(\mathrm{~L})$. The light green bands add information from measurements with both beams polarized for the values $\left(P_{e^{+}}, P_{e^{-}}\right)=(-60 \%,+90 \%)(\mathrm{LR})$ and $(+60 \%,+90 \%)(\mathrm{RR})$. Combining all constraints leads to the dark red region. The errors correspond to an integrated luminosity of $100 \mathrm{fb}^{-1}$ for each polarization combination [86]. The SUSY parameters are chosen as in scenario S2, table 3.1.

different combinations of $\tilde{e}_{\mathrm{R}}$ and $\tilde{e}_{\mathrm{L}}$ can only be distinguished by the initial beam polarization. If one provides the relative contributions of the different produced selectron pairs from theory, the use of electron polarization alone would be sufficient to measure both the SU(2) and U(1) SUSY Yukawa couplings.

Without using this theoretical information, it is necessary to have both beams polarized for a measurement of the Yukawa couplings. This is illustrated in fig. 3.3 for scenario $\mathrm{S} 2$, cf. table 3.1. The use of only $e^{-}$beam polarization leaves a four-fold ambiguity in the determination of the Yukawa couplings, which can be resolved by including cross-section measurements with simultaneous polarization of the $e^{+}$and $e^{-}$beams. Combining this information, the $\mathrm{U}(1)$ and $\mathrm{SU}(2)$ Yukawa couplings can be determined with a precision of $0.2 \%$ and $1.2 \%$, respectively, see fig. 3.3. The results shown take into account the selectron decay distributions, including SM and SUSY backgrounds that have been reduced by appropriate cuts, beamstrahlung, ISR and the most important systematic uncertainties (see [85] for details).

Quantitative example: The above analysis shows that even an extremely high degree of electron polarization, say $P_{e^{-}} \geq 90 \%$, would be insufficient to test the chiral quantum numbers associated to the scalar $\tilde{e}^{ \pm}$. Also, a measurement of the Yukawa couplings, which is important to prove their equality to the gauge couplings in SUSY, 
might not succeed if only polarized electrons were available.

\subsubsection{Smuon mass measurement above the production threshold}

Smuon production occurs only via $\gamma$ and $Z$ exchange. In order to measure the masses, the suppression of the SM $W W$ process is necessary. The polarization of both beams may be decisive to observe all kinematical edges and measure $m_{\tilde{\mu}_{L, R}}$ in the continuum. This is also important to optimize threshold scans.

Smuons are only pair-produced via $\gamma, Z$ exchange in the $s$-channel, $e^{+} e^{-} \rightarrow \tilde{\mu}_{L, R}^{+} \tilde{\mu}_{L, R}^{-}$, cf. fig. 3.1. The production process depends at tree-level only on the mass parameters of the scalar sector. In threshold scans at the linear collider the smuon masses can be determined very precisely to the order of a few $100 \mathrm{MeV}$ [1]. Since, however, threshold scans need running time, i.e. it requires luminosity specific around this energy, it is important to measure the masses already rather precisely far beyond the threshold, in the continuum. To achieve a precision measurement it is important to suppress efficiently background processes. For smuon production, $W^{+} W^{-}$final states represent one of the worst backgrounds; however, they can easily be suppressed with right-handed electron/left-handed positron beams. The scaling factors are listed in table 2.4, see section 2.2.

The background suppression may be decisive for the detection of the new particles and the accurate measurement of their masses $m_{\tilde{\mu}}$ in the continuum. Since $\tilde{\mu}^{+} \tilde{\mu}^{-}$production only proceeds via $s$-channel $\gamma, Z$ exchange, the initial beam configurations LR $\left(P_{e^{-}}<0, P_{e^{+}}>0\right)$ and RL $\left(P_{e^{-}}>0, P_{e^{+}}<0\right)$ are favoured. A case study has been made [87] for the scenario S3, cf. table 3.1, where the masses are

$$
m_{\tilde{\mu}_{R}}=178.3 \mathrm{GeV}, \quad m_{\tilde{\mu}_{L}}=287.1 \mathrm{GeV} .
$$

\begin{tabular}{|c|c|c|c|}
\hline Polarization & $\sigma\left(\tilde{\mu}_{R}^{+} \tilde{\mu}_{R}^{-}\right)[\mathrm{fb}]$ & $\sigma\left(\tilde{\mu}_{L}^{+} \tilde{\mu}_{L}^{-}\right)[\mathrm{fb}]$ & $\sigma\left(W^{+} W^{-}\right)[\mathrm{fb}]$ \\
\hline$(-80 \%,-80 \%)$ & 11.44 & 5.06 & 1448 \\
\hline$(-80 \%,+80 \%)$ & 21.23 & 37.74 & 12995 \\
\hline$(+80 \%,-80 \%)$ & 82.99 & 8.37 & 198 \\
\hline$(+80 \%,+80 \%)$ & 11.44 & 5.06 & 1448 \\
\hline \hline$(-80 \%, 0)$ & 16.34 & 21.40 & 7241 \\
\hline$(+80 \%, 0)$ & 47.21 & 6.72 & 824 \\
\hline
\end{tabular}

Table 3.2: Cross sections for $e^{+} e^{-} \rightarrow \tilde{\mu}^{+} \tilde{\mu}^{-}$at $\sqrt{s}=750 \mathrm{GeV}$ for the scenario S3 [87]. One observes a large reduction of the $W^{+} W^{-}$cross section when the electron is right-handed and the positron is left-handed. This helps significantly in observing $\tilde{\mu}_{L}$.

The cross sections are shown in table 3.2, where the considerable reduction in the $W W$ production cross section for right-handed electrons and left-handed positrons can be noted. One gains a factor of about 2.6 in the ratio $S / \sqrt{B}$ with $\left(P_{e^{-}}, P_{e^{+}}\right)=(+80 \%,-80 \%)$ compared to $(+80 \%, 0)$. In fig. 3.4 the expected muon energy distribution is shown for an integrated luminosity of $500 \mathrm{fb}^{-1}$ at $\sqrt{s}=750 \mathrm{GeV}$ and for the polarization configurations $\left(P_{e^{-}}, P_{e^{+}}\right)=(-80 \%,+80 \%)$ (left panel) and $(+80 \%,-80 \%)$ (right panel). The background 
from $W^{+} W^{-}$decaying into the $\mu \nu$ final state is included. Only with polarized $e^{-}$and $e^{+}$ beams can both muon-energy edges, at around 65 and $220 \mathrm{GeV}$, be reconstructed. The slepton masses can be determined in the continuum up to a few $\mathrm{GeV}$ uncertainty. This shows the real importance of positron polarization for a clear observation of the lowenergy edge associated to the $\tilde{\mu}_{R}$, which cannot be clearly seen unless the positron is polarized [87].
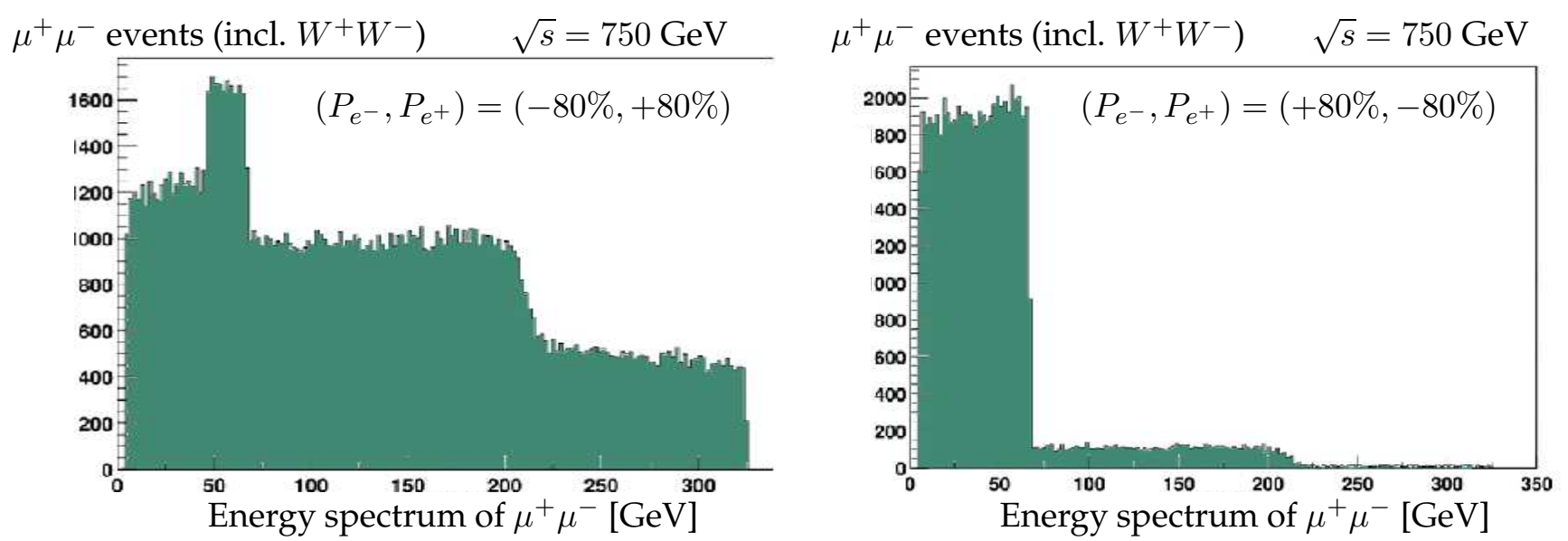

Figure 3.4: Energy spectrum of muons from $\tilde{\mu}_{L, R}$ decays into $\mu \tilde{\chi}_{1}^{0}$ final states, including the $W^{+} W^{-}$background decaying into $\mu \nu$ final states in the scenario S3, cf. table 3.1, for two combinations of beam polarizations for $\sqrt{s}=750 \mathrm{GeV}$ and $\mathcal{L}_{\text {int }}=500 \mathrm{fb}^{-1}$ [87].

Quantitative examples: The most important background to $\tilde{\mu}$ pair production is $W W$ pair production. Compared with the case of only the electron beam polarized, the signal gains about a factor $\mathbf{1 . 8}$ and the background is suppressed by about a factor of 4 with $\left(P_{e^{-}}, P_{e^{+}}\right)=(+80 \%,-80 \%)$ compared to $(+80 \%, 0)$. With both beams polarized, a rather accurate measurement of the smuon masses is possible already in the continuum, which can then be used to devise possible threshold scans.

\subsubsection{Determination of third-generation sfermion parameters}

The advantages of having both beams polarized in third-generation sfermion production are the larger cross sections and a more precise determination of masses and mixing angles.

In the third generation of sfermions, Yukawa terms give rise to a mixing between the 'left' and 'right' states $\tilde{f}_{\mathrm{L}}$ and $\tilde{f}_{\mathrm{R}}(\tilde{f}=\tilde{t}, \tilde{b}, \tilde{\tau})$. The mass eigenstates are $\tilde{f}_{1}=\tilde{f}_{\mathrm{L}} \cos \theta_{\tilde{f}}+$ $\tilde{f}_{\mathrm{R}} \sin \theta_{\tilde{f}}$, and $\tilde{f}_{2}=\tilde{f}_{\mathrm{R}} \cos \theta_{\tilde{f}}-\tilde{f}_{\mathrm{L}} \sin \theta_{\tilde{f}}$, with $\theta_{\tilde{f}}$ the sfermion mixing angle.

In the following phenomenological studies of third-generation sfermions in $e^{+} e^{-}$annihilation at $\sqrt{s}=500 \mathrm{GeV}$ are summarized. Information on the mixing angle can be obtained by measuring production cross sections with different combinations of beam polarizations. It has been shown in $[88,90,91]$ that beam polarization is important to resolve ambiguities, see fig. 3.5. For the unpolarized case, two values of $\cos 2 \theta_{\tilde{\tau}}\left(\theta_{\tilde{\tau}}\right.$ being the mixing angle) are consistent with the cross sections (red lines). However, the use of polarized beams allows a single solution (green and blue lines) to be identified. Moreover, 
the simultaneous polarization of both beams is useful for enhancing $P_{\text {eff, }}$ eq. (1.19), together with the signal and thus for reducing uncertainties [91]. This might be particularly important for $\tilde{\tau} / \tau$ analyses, which will be difficult to perform, since $\tau$ s decay also into $2 \pi$ and $3 \pi$ final states. In [91] a complete detector simulation of signal and background (using the program SIMDET [48]) for the scenario S4, table3.1, including angle cuts, QED radiation and beamstrahlung and using $\left(P_{e^{-}}, P_{e^{+}}\right)=(+80 \%,-60 \%)$, has been performed. The result was that $m_{\tilde{\tau}_{1}}$ could even be measured to a precision of $500 \mathrm{MeV}$, the mixing angle $\cos 2 \theta_{\tilde{\tau}}$ up to $1 \%$ and the polarization of the $\tau, P_{\tau}$, up to $6 \%$ at the ILC. Compared to the case with only polarized electrons of $\left|P_{e^{-}}\right|=80 \%$ the polarization of both beams leads in the determination of $m_{\tilde{\tau}_{1}}$ and $P_{\tau}$ approximately to a reduction of the uncertainty by about a factor 1.6. Since the determination of the mixing angle $\cos 2 \theta_{\tilde{\tau}}$ is mainly determined by systematics positron polarization leads in this context only to an improvement of $\mathrm{O}(10 \%)$.

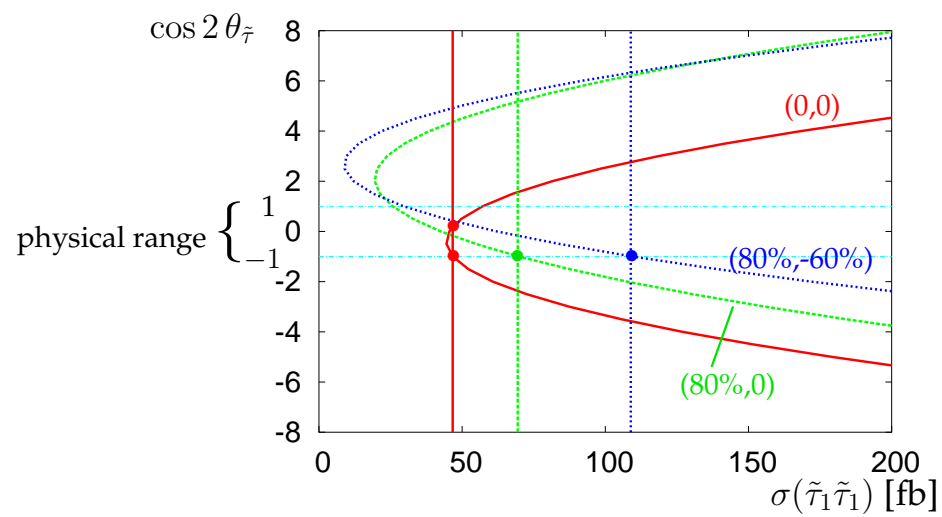

Figure 3.5: Mixing parameter $\cos 2 \theta_{\tilde{\tau}}$ vs. cross section $\sigma\left(e^{+} e^{-} \rightarrow \tilde{\tau}_{1} \tilde{\tau}_{1}\right)$ at $\sqrt{s}=500 \mathrm{GeV}$ for beam polarizations as indicated. The vertical lines indicate the predicted cross sections [91]. The SUSY parameters are chosen as in scenario S4, cf. table 3.1.

In the following, the production of light stops is discussed in detail. The reaction $e^{+} e^{-} \rightarrow \tilde{t}_{i} \tilde{\tilde{t}}_{j}, i, j=1,2$ proceeds via $\gamma$ and $Z$ exchange in the $s$-channel. The $\tilde{t}_{i}$ couplings depend on the stop-mixing angle $\theta_{\tilde{t}}$. In Figs. $3.6 \mathrm{a}, \mathrm{b}$, contour lines of the cross section $\sigma\left(e^{+} e^{-} \rightarrow \tilde{t}_{1} \overline{\tilde{t}}_{1}\right)$ are shown as functions of the beam polarizations $P_{e^{-}}$and $P_{e^{+}}$at $\sqrt{s}=$ $500 \mathrm{GeV}$ for $m_{\tilde{t}_{1}}=200 \mathrm{GeV}$, with (a) $\cos \theta_{\tilde{t}}=0.4$ and (b) $\cos \theta_{\tilde{t}}=0.66$, see scenario S5, table 3.1. Initial-state radiation (ISR) and one-loop SUSY-QCD corrections are included (for details, see $[88,90]$ ). The white windows show the range of polarizations $\left|P_{e^{-}}\right|<0.9$ and $\left|P_{e^{+}}\right|<0.6$. As can be seen, one significantly increases the cross section by up to a factor of about 1.6 with $\left(P_{e^{-}}, P_{e^{+}}\right)=(+90 \%,-60 \%)$ compared to $(+90 \%, 0)$. Moreover, beam polarization strengthens the $\cos \theta_{\tilde{t}}$ dependence and can thus be essential for the determination of the mixing angle. Corresponding cross sections for the production of sbottoms, staus and $\tau$-sneutrinos are presented in [88].

The precision that can be obtained from cross section measurements on the parameters of the stop sector is estimated using the parameter point S5, table 3.1 , with $m_{\tilde{t}_{1}}=200 \mathrm{GeV}$ and $\cos \theta_{\tilde{t}}=-0.66$ as an illustrative example. The four cases $\left(P_{e^{-}}, P_{e^{+}}\right)=(\mp 0.9,0)$ and $\left(P_{e^{-}}, P_{e^{+}}\right)=(\mp 0.9, \pm 0.6)$ are studied with integrated luminosities of $\mathcal{L}_{\text {int }}=50 \mathrm{fb}^{-1}$ and $\mathcal{L}_{\text {int }}=250 \mathrm{fb}^{-1}$ for each polarization. Again, one-loop SUSY-QCD and ISR corrections are included. A $1 \%$ uncertainty in the polarizations of the $e^{+}$and $e^{-}$beams has been assumed as well as a theoretical uncertainty in the cross sections of $1 \%$, taking full error 

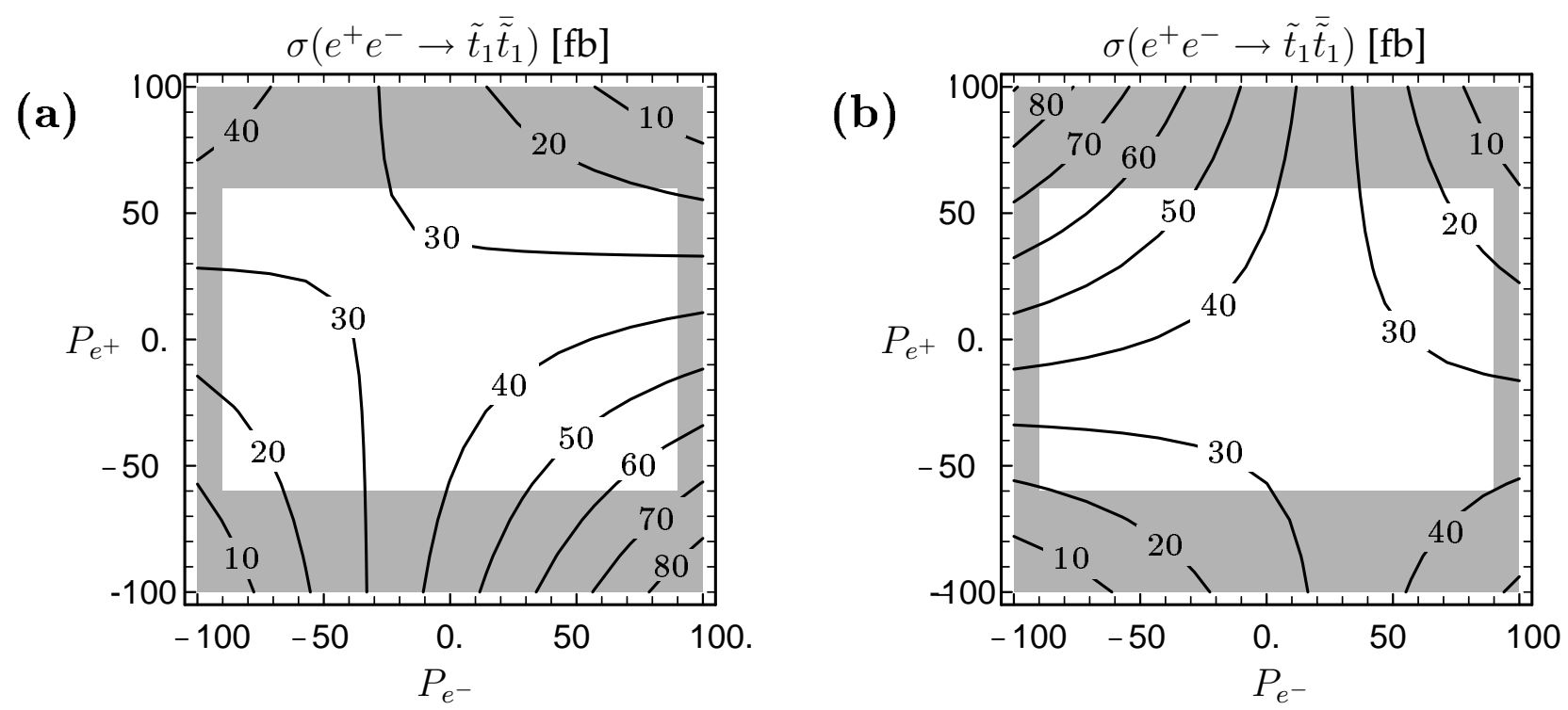

Figure 3.6: Dependence of $\sigma\left(e^{+} e^{-} \rightarrow \tilde{t}_{1} \tilde{\bar{t}}_{1}\right)$ on degree of electron and positron polarization at $\sqrt{s}=500 \mathrm{GeV}$, for $m_{\tilde{t}_{1}}=200 \mathrm{GeV},\left|\cos \theta_{\tilde{t}}\right|=0.4$ in (a) and $\left|\cos \theta_{\tilde{t}}\right|=0.66$ in (b), cf. scenario S5, table 3.1 [88].

propagation into account. The anticipated experimental precisions on $\sigma_{-+}$and $\sigma_{+-}$are based on the Monte Carlo study of [92]. Fig. 3.7a shows the error bands for the cross sections and the corresponding 68\% CL error ellipses in the $m_{\tilde{t}_{1}}-\cos \theta_{\tilde{t}}$ plane for the four cases mentioned above.

The mixing angle can be determined even more precisely from the left-right asymme$\operatorname{try} A_{\mathrm{LR}}^{\mathrm{obs}}=\left(\sigma_{-+}-\sigma_{+-}\right) /\left(\sigma_{-+}+\sigma_{+-}\right)$, cf. eq. (1.24), because here the kinematical dependence on $m_{\tilde{t}_{1}}$ drops out. The error bands for $A_{L R}$ for the four cases as a function of $\cos \theta_{\tilde{t}}$ are shown in Fig. 3.7b. Table 3.3 summarizes the cross sections and statistical errors as well as the resulting errors on $m_{\tilde{t}_{1}}$ and $\cos \theta_{\tilde{t}}[89]$.

\begin{tabular}{c|ccccccc|ccc} 
case & $\mathcal{L}_{\text {int }}$ & $P_{e^{-}}$ & $P_{e^{+}}$ & $\sigma_{-+}$ & $\sigma_{+-}$ & $\Delta \sigma_{-+}^{\text {stat. }}$ & $\Delta \sigma_{+-}^{\text {stat. }}$ & $\Delta m_{\tilde{t}_{1}}$ & $\begin{array}{r}\text { Fig. 3.7a } \\
\Delta \cos \theta_{\tilde{t}}\end{array}$ & $\begin{array}{c}\text { Fig. 3.7b } \\
\end{array}$ \\
\hline 1 & $100 \mathrm{fb}^{-1}$ & $\mp 0.9$ & 0 & $44 \mathrm{fb}$ & $27 \mathrm{fb}$ & $4.7 \%$ & $6.3 \%$ & $1.1 \%$ & $3.6 \%$ & $2.3 \%$ \\
2 & $500 \mathrm{fb}^{-1}$ & $\mp 0.9$ & 0 & $44 \mathrm{fb}$ & $27 \mathrm{fb}$ & $2.1 \%$ & $2.8 \%$ & $0.5 \%$ & $1.8 \%$ & $1.1 \%$ \\
3 & $100 \mathrm{fb}^{-1}$ & $\mp 0.9$ & \pm 0.6 & $69 \mathrm{fb}$ & $40 \mathrm{fb}$ & $3.1 \%$ & $4.4 \%$ & $0.8 \%$ & $2.3 \%$ & $1.4 \%$ \\
4 & $500 \mathrm{fb}^{-1}$ & $\mp 0.9$ & \pm 0.6 & $69 \mathrm{fb}$ & $40 \mathrm{fb}$ & $1.4 \%$ & $2.0 \%$ & $0.4 \%$ & $1.1 \%$ & $0.7 \%$
\end{tabular}

Table 3.3: Parameters for the cases studied, cross sections, assumed statistical errors and resulting precisions on $m_{\tilde{t}_{1}}$ and $\cos \theta_{\tilde{t}}$ corresponding to Fig. 3.7 [89] and SUSY scenario S5, cf. table 3.1.

Quantitative example: In the cases studied in this section, the cross sections are en- 

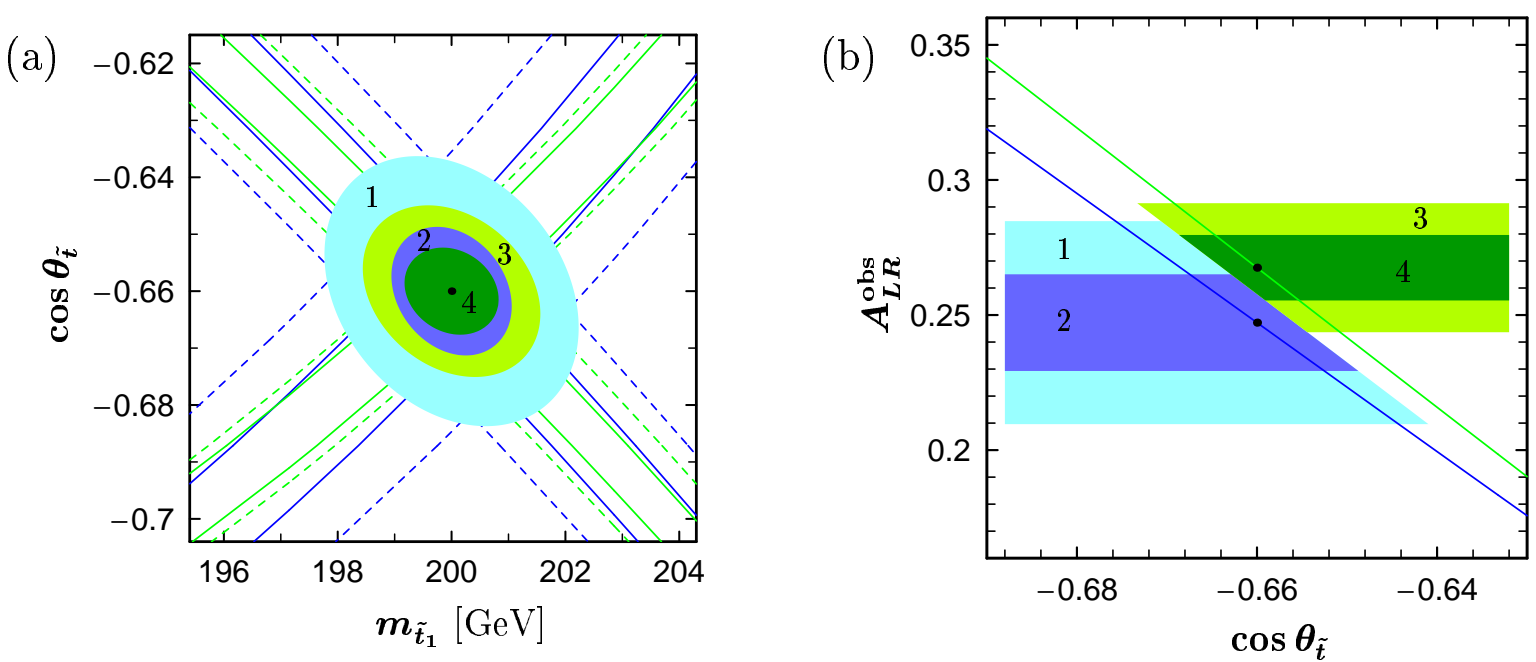

Figure 3.7: (a) Cross section error bands and 68\% CL error ellipses for determining $m_{\tilde{t}_{1}}$ and $\cos \theta_{\tilde{t}}$ from cross section measurements for the four cases given in Table 3.3; the dashed lines are for $\mathcal{L}_{\text {int }}=100 \mathrm{fb}^{-1}$ and the full lines for $\mathcal{L}_{\text {int }}=500 \mathrm{fb}^{-1}$, the blue (green) lines are for cases 1 and 2 (cases 3 and 4). (b) Error bands for the determination of $\cos \theta_{\tilde{t}}$ from $A_{\mathrm{LR}}^{\text {obs }}$. In both plots $m_{\tilde{t}_{1}}=200 \mathrm{GeV}, \cos \theta_{\tilde{t}}=-0.66, \sqrt{s}=500 \mathrm{GeV}$. [89]. The SUSY parameters are chosen as in scenario S5, cf. table 3.1.

hanced by a factor of about 1.6 with both beams polarized, $\left(\left|P_{e^{-}}\right|,\left|P_{e^{+}}\right|\right)=(90 \%, 60 \%)$, as compared to the case with only $\left(\left|P_{e^{-}}\right|, P_{e^{+}}\right)=(90 \%, 0)$. This gives up to $40 \% \mathrm{im}$ provement in the determination of the stop mass and mixing angle.

\subsubsection{Chargino and neutralino production}

In chargino and neutralino production, a complicated interplay takes place between the $s$-channel amplitudes and the amplitudes originating from scalar exchange in the $t$ - and $u$-channels. Consequently, only polarized electrons may not be sufficient for a model-independent determination of the relevant MSSM parameters, and positron polarization would also be needed.
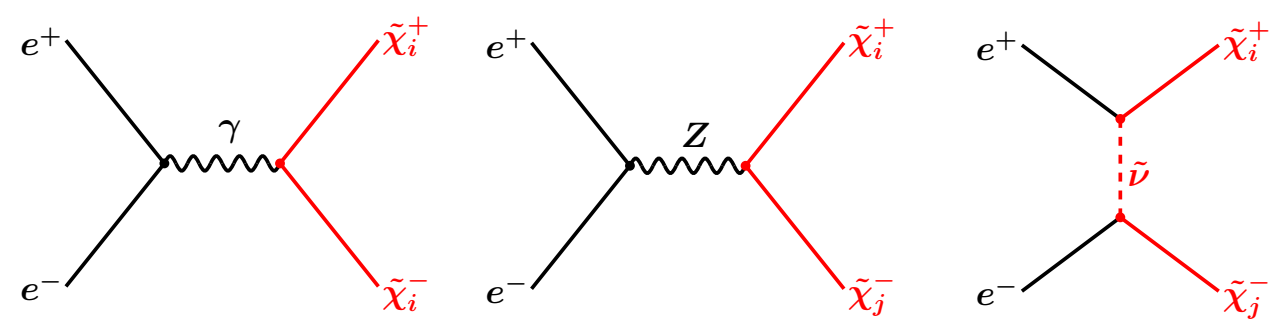

Figure 3.8: Chargino production: $\gamma, Z$-exchange in the $s$-channel and $\tilde{\nu}_{e}$-exchange in the $t$-channel.

In the following the fermionic sector of the charginos, $\tilde{\chi}_{1,2}^{ \pm}$, and neutralinos, $\tilde{\chi}_{1, \ldots, 4}^{0}$ is studied. It is expected that at least some of the charginos and neutralinos are sufficiently light to be directly observed at $\sqrt{s}=500 \mathrm{GeV}$. Since these particles are mixtures of the 


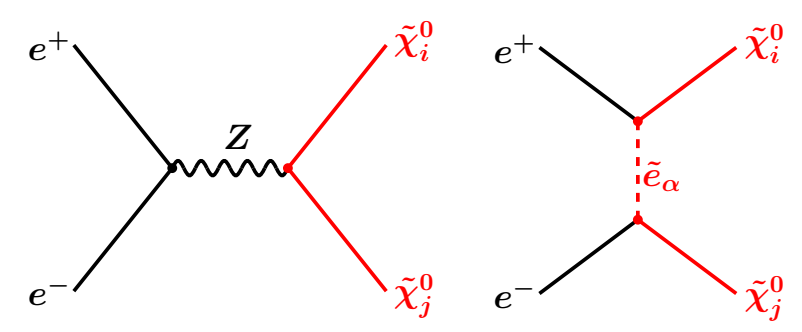

Figure 3.9: Neutralino production: $Z$-exchange in the $s$-channel and $\tilde{e}_{L}, \tilde{e}_{R}$-exchange in the $t$ - and $u$-channel.

gaugino and higgsino interaction states their analysis will give direct access to the SUSY breaking parameters $M_{1}, M_{2}, \mu$ and $\tan \beta$ [93]. Beam polarization may be useful for the determination of the parameters and couplings of the gaugino/higgsino sector, in particular if only part of the spectrum turns out to be kinematically accessible [94].

Charginos (neutralinos) are produced in pairs via exchange of the $\gamma, Z$ (Z) gauge bosons in the $s$-channel and via scalar SUSY particle exchange, $\tilde{\nu}_{e}\left(\tilde{e}_{L, R}\right)$, in the $t$-channel ( $t$ - and $u$-channel), see figs. 3.8 and 3.9. The production processes depends therefore at tree-level on the parameters $M_{2}, \mu, \tan \beta$ (in the case of $\tilde{\chi}_{j}^{0}$ : also on $M_{1}$ ) and on the mass parameters of the exchanged scalar particles. If the beams were fully polarized, only the LR and RL beam-polarization configurations would contribute to the production processes. The cross sections in the configurations LL and RR are non-vanishing for partially polarized beams. Since charginos and neutralinos are mixtures of gauginos (which couple only to scalar SUSY partners $\tilde{\nu}_{e}$ and $\tilde{e}_{L, R}$, respectively) and higgsinos (which couple only to the $Z$ boson in the $s$-channel) interesting features can be exposed when applying all possible polarization configurations for the different chargino and neutralino pair productions [95].

Beam polarization can significantly enhance the signal and therefore improve the $S / \sqrt{B}$ ratio. In the case of chargino pair production, as can be seen in the left panel of fig. 3.10 (for the scenario S6, see table 3.1) the cross section for $(-90 \%,+60 \%)$ is enhanced by a factor of 1.6 compared to the $(-90 \%, 0)$ case. For right-polarized electrons the enhancement is much weaker in this scenario. Nevertheless, polarized beams can be used to disentangle the chargino and sneutrino parameters, exploiting the fact that the $\tilde{\nu}_{e}$ exchange affects only the amplitude with left-chiral electrons (and right-chiral positrons). If the incoming beams were $100 \%$ polarized, one could switch the $\tilde{\nu}_{e}$ exchange on and off, and analyse the chargino system alone or with $\tilde{\nu}_{e}$ included, and thus perform an independent determination of chargino and $\tilde{\nu}_{e}$ parameters. With realistic beam polarization, the $\tilde{\nu}_{e}$ exchange affects all amplitudes depending on the degree of polarization, as seen in fig. 3.10. Consequently, the polarization of both beams provides a unique analysing tool.

The case of neutralinos is even more interesting since the structure in the $t$ - and $u$ channels is richer. As can be seen in fig. 3.11a for S6, see table 3.1, the cross sections for pair production are also enhanced by a factor 1.6 for $(-90 \%,+60 \%)$ with respect to the case $(-90 \%, 0)$. For right-polarized electrons, similar results are obtained, see e.g. $\sigma\left(\tilde{\chi}_{3}^{0} \tilde{\chi}_{4}^{0}\right)$, in fig. 3.11b. In this configuration an even greater advantage of polarizing both beams with different signs is the suppression of the dominant $W W$ background. One should note, however, that the obtained enhancement can strongly depend on the considered scenario, see table 3.4 . 

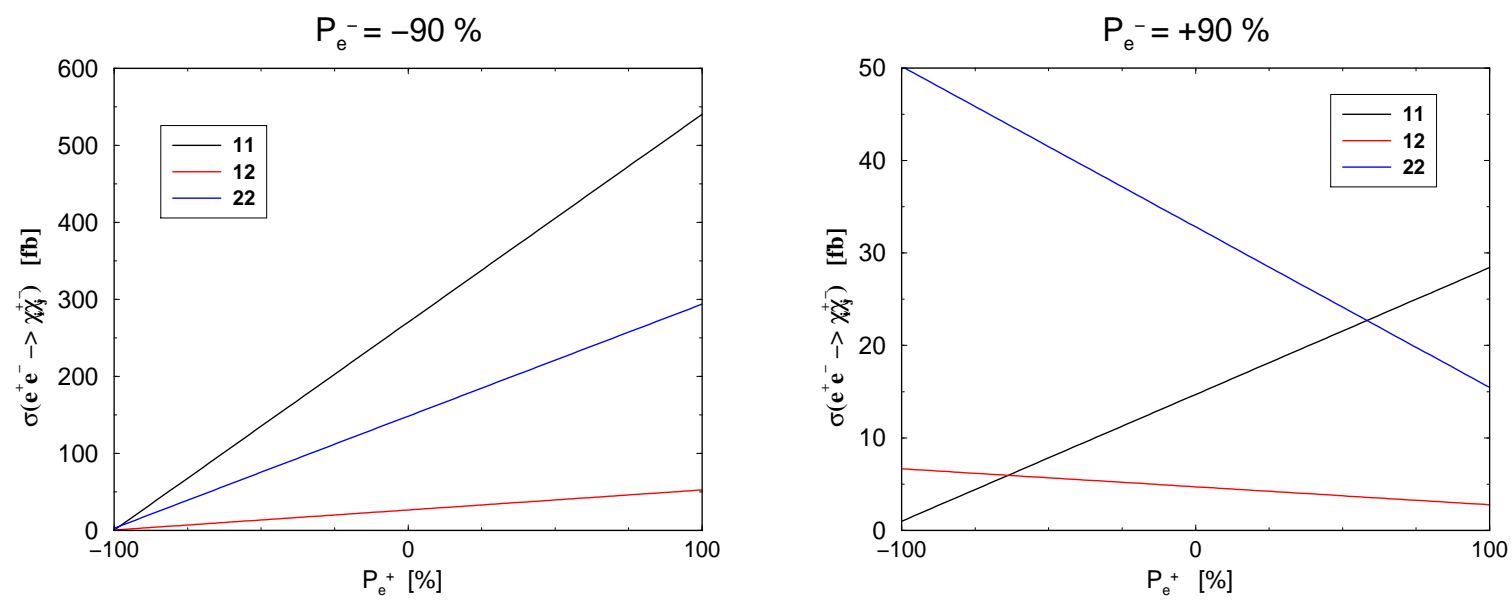

Figure 3.10: Chargino production: cross sections for $e^{+} e^{-} \rightarrow \tilde{\chi}_{i}^{-} \tilde{\chi}_{j}^{+}$at $\sqrt{s}=1 \mathrm{TeV}$ for fixed electron polarization $P_{e^{-}}=-90 \%$ (left) and $P_{e^{-}}=+90 \%$ (right) as a function of positron polarization [96]. The SUSY parameters are chosen as in scenario S6, see table 3.1.
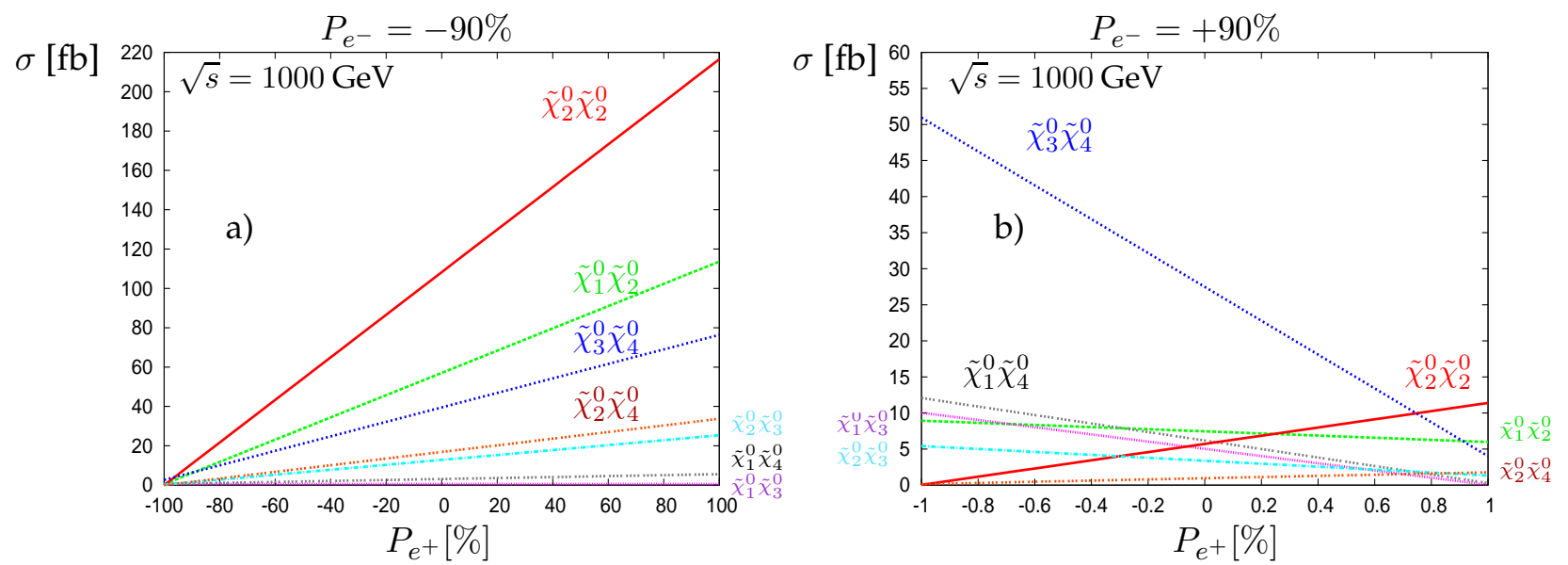

Figure 3.11: Neutralino production: Selected cross sections for $e^{+} e^{-} \rightarrow \tilde{\chi}_{i}^{0} \tilde{\chi}_{j}^{0}$ at $\sqrt{s}=1 \mathrm{TeV}$ in the scenario 56 , see table 3.1, where the two lightest neutralinos are gaugino-like and the two heavier ones are higgsino-like, shown for fixed electron polarization and variable positron polarization, a) $P_{e^{-}}=-90 \%$, and b) $P_{e^{-}}=+90 \%$ [97].

Particularly interesting is the behaviour of the $\tilde{\chi}_{2}^{0} \tilde{\chi}_{2}^{0}$ cross section, which is enhanced by a factor of 1.6 even for the exceptional configuration $(+90 \%,+60 \%)$ with respect to the case $(+90 \%, 0)$. This effect is due to the lack of mixing, the $\tilde{\chi}_{2}^{0}$ being nearly pure wino, and thus not completely suppressed for partial polarization $(+90 \%,+60 \%)$. In the case of completely polarized beams, the RR cross section for $\tilde{\chi}_{2}^{0} \tilde{\chi}_{2}^{0}$ would vanish, since-as mentioned before-only a V-A interaction occurs in this process.

Neutralino production is very sensitive also to the mass of the exchanged particles, $\tilde{e}_{\mathrm{L}}$ and $\tilde{e}_{\mathrm{R}}$. The ordering of the cross sections for different polarization configurations (with $\left.\left(\left|P_{e^{-}}\right|,\left|P_{e^{+}}\right|\right)=(80 \%, 60 \%)\right)$ depends on the character of the neutralinos as well as on the masses of the exchanged particles:

- Pure higgsino (only s-channel): 


$$
\sigma_{-+}>\sigma_{+-}>\sigma_{-0}>\sigma_{00}>\sigma_{+0}>\sigma_{--}>\sigma_{++}
$$

- Pure gaugino and $m_{\tilde{e}_{\mathrm{L}}} \gg m_{\tilde{e}_{\mathrm{R}}}$ (only $t$ - and $u$-channels):

$$
\sigma_{+-}>\sigma_{+0}>\sigma_{00}>\sigma_{++}>\sigma_{--}>\sigma_{-0}>\sigma_{-+}
$$

- Pure gaugino and $m_{\tilde{e}_{\mathrm{L}}} \ll m_{\tilde{e}_{\mathrm{R}}}$ (only $t$ - and $u$-channels):

$$
\sigma_{-+}>\sigma_{-0}>\sigma_{00}>\sigma_{--}>\sigma_{++}>\sigma_{+0}>\sigma_{+-},
$$

with denotation as defined in eq. (1.15), sect.1.2.2. If only the electron beam is polarized, then the orderings of the cross sections for the cases (3.3) and (3.5) remain the same. If, however, both beams are simultaneously polarized, the three cases could be distinguished from one another [95].

If the slepton masses are known, the fundamental SUSY parameters, $M_{1}, M_{2}, \mu$ and $\tan \beta$ can easily be determined from measurements of polarized cross sections of the two lightest neutralinos and the lightest chargino [94]. To give an example of the involved interplay between gaugino/higgsino mixing character, the influence of the virtual slepton masses and the dependence of beam polarization, polarized cross sections $\sigma\left(e^{+} e^{-} \rightarrow \tilde{\chi}_{1}^{0} \tilde{\chi}_{2}^{0}\right)$ for different $m_{\tilde{e}_{L, R}}$ are listed in table 3.4 for the SUSY parameters chosen as in scenario S7, see table 3.1.

Considering the large number of possible independent SUSY parameters, the availability of different observables using the polarization of both beams is a decisive tool for the kind of analysis described here.

\begin{tabular}{|c|c|cc|cc|}
\hline $\begin{array}{c}\left(m_{\tilde{e}_{\mathrm{L}}}, m_{\tilde{e}_{\mathrm{R}}}\right) \\
{[\mathrm{GeV}]}\end{array}$ & $\begin{array}{c}\sigma\left(e^{+} e^{-} \rightarrow \tilde{\chi}_{1}^{0} \tilde{\chi}_{2}^{0}\right) \\
{[\mathrm{fb}] \text { unpolarized }}\end{array}$ & $(-90 \%, 0 \%)$ & $(+90 \%, 0 \%)$ & $(-90 \%,+60 \%)$ & $(+90 \%,-60 \%)$ \\
\hline$(200,200)$ & 102 & 0.4 & 1.6 & 0.6 & 2.5 \\
$(500,500)$ & 29 & 0.5 & 1.5 & 0.7 & 2.4 \\
$(1000,1000)$ & 7.4 & 0.7 & 1.3 & 1.1 & 2.0 \\
$(1500,1500)$ & 4.0 & 0.9 & 1.1 & 1.3 & 1.8 \\
$(2000,2000)$ & 2.9 & 1.0 & 1.0 & 1.5 & 1.6 \\
$(500,1500)$ & 8.7 & 1.4 & 0.6 & 2.3 & 0.8 \\
$(1500,500)$ & 24 & 0.2 & 1.8 & 0.3 & 2.8 \\
\hline
\end{tabular}

Table 3.4: Polarized cross sections $\sigma\left(e^{+} e^{-} \rightarrow \tilde{\chi}_{1}^{0} \tilde{\chi}_{2}^{0}\right)$ at $\sqrt{s}=500 \mathrm{GeV}$ with all possible beam-polarization configurations for $\left(P_{e^{-}}, P_{e^{+}}\right)=(\mp 90 \%, \pm 60 \%)$ and for different selectron masses $m_{\tilde{e}_{L, R}}$, cf. [97]. The SUSY parameters are given by scenario S7, table 3.1. Cross sections in the configuration RR and LL are $<1 \mathrm{fb}$ and not listed.

Quantitative examples: One can gain up to a factor of about 1.8 for the production cross section with both beams polarized with respect to the case where only electrons are polarized. To determine the fundamental parameters model-independently it is necessary to observe cross sections with differently polarized beams. Parameters might be such that having only one beam polarized would not unambiguously determine the parameters. 


\subsubsection{CP violation in neutralino production and decay}

With both beams polarized, the CP-violating effects can be enhanced by a considerable factor, with respect to the case of only electrons polarized.

Particularly interesting in supersymmetry is the study of new CP-violating sources. In the following, suitable observables for exploring CP violation in the gaugino/higgsino sector are shown.

The interaction Lagrangian for the neutralino and chargino sectors of the MSSM, see e.g. [98], allows for some SUSY parameters to be complex: the gaugino mass parameter $M_{1}$ ( $M_{2}$ is assumed to be real), the higgsino mass parameter $\mu$, and -involving third generation sfermions - the trilinear coupling parameter $A_{\tau}$ in the stau sector,

$$
\mu=|\mu| e^{i \varphi_{\mu}}, \quad M_{1}=\left|M_{1}\right| e^{i \varphi_{M_{1}}}, \quad A_{\tau}=\left|A_{\tau}\right| e^{i \varphi_{A_{\tau}}} .
$$

The physical phases can cause large CP-violating effects already at tree level [98-108]. In the following, the dependence on the beam polarization of these $\mathrm{CP}$-violating effects in neutralino production and decay is reviewed [109].

In the following neutralino production

$$
e^{+}+e^{-} \rightarrow \tilde{\chi}_{2}^{0}+\tilde{\chi}_{j}^{0}, \quad j=1,3
$$

with either leptonic two-body decays

$$
\tilde{\chi}_{2}^{0} \rightarrow \tilde{\ell}+\ell_{1} \text { and } \tilde{\ell} \rightarrow \tilde{\chi}_{1}^{0}+\ell_{2}
$$

or the corresponding leptonic three-body decays

$$
\tilde{\chi}_{2}^{0} \longrightarrow \tilde{\chi}_{1}^{0}+\ell_{1}+\ell_{2},
$$

with $\ell_{1,2}=e, \mu, \tau$ is studied. The spin correlations between production and decays of the neutralinos lead to CP-violating effects already at tree level and allow the definition of several CP-odd asymmetries. In the following only the decays of the $\tilde{\chi}_{2}^{0}$ are studied.

It is convenient to introduce the triple product $\mathcal{T}=\left(\mathbf{p}_{e^{-}} \times \mathbf{p}_{\ell_{2}}\right) \cdot \mathbf{p}_{\ell_{1}}$, where $\mathbf{p}_{e^{-}}, \mathbf{p}_{\ell_{1}}$ and $\mathbf{p}_{\ell_{2}}$ are the momenta of the initial $e^{-}$beam and the two final leptons $\ell_{1}$ and $\ell_{2}$, respectively. A T-odd asymmetry of the cross section $\sigma$ for the processes (3.7)-(3.9) can then be defined as:

$$
\mathcal{A}_{\mathrm{T}}=\frac{\sigma(\mathcal{T}>0)-\sigma(\mathcal{T}<0)}{\sigma(\mathcal{T}>0)+\sigma(\mathcal{T}<0)}=\frac{\int \operatorname{sign}\{\mathcal{T}\}|\mathcal{M}|^{2} d \text { Lips }}{\int|\mathcal{M}|^{2} d \text { Lips }}
$$

If final-state phases and finite width effects of exchanged particles are neglected, $\mathcal{A}_{\mathrm{T}}$ is $\mathrm{CP}$ violating $\left(\mathrm{CP}\right.$-odd) due to $\mathrm{CPT}$ invariance. $\mathcal{A}_{\mathrm{T}}$ is proportional to the difference between the number of events with the final lepton $\ell_{1}$ above the plane spanned by $\mathbf{p}_{e^{-}}$and $\mathbf{p}_{\ell_{2}}$ and those below it. The dependence of $\mathcal{A}_{\mathrm{T}}$ on $\varphi_{M_{1}}$ and $\varphi_{\mu}$ has been analysed in [98-100,104, 107], see sections a) and b) below.

When the neutralino decays into a $\tau$ lepton,

$$
\tilde{\chi}_{i}^{0} \rightarrow \tilde{\tau}_{k}^{ \pm} \tau^{\mp}, \quad k=1,2,
$$

the transverse $\tau^{-}$and $\tau^{+}$polarizations $P_{\tau^{-}}$and $P_{\tau^{+}}$, perpendicular to the plane formed by the $\tau$ and $e^{-}$momenta, are T-odd and give rise to the CP-odd observable

$$
\mathcal{A}_{\mathrm{CP}}=\frac{1}{2}\left(P_{\tau^{-}}-P_{\tau^{+}}\right),
$$


which is also sensitive to $\varphi_{A_{\tau}}$. $\mathcal{A}_{\mathrm{CP}}$ has been discussed in [104,105] for several MSSM scenarios. In order to measure the asymmetries $\mathcal{A}_{\mathrm{T}}$ and $\mathcal{A}_{\mathrm{CP}}$ in the leptonic two-body decays of the neutralino, eq. (3.8), the lepton $\ell_{1}$ from the neutralino decay and the lepton $\ell_{2}$ from the $\tilde{\ell}$ decay, have to be distinguished. have to be distinguished. This can be accomplished by measuring the different energy spectra of the $\tau \mathrm{s}$ [98].

To measure the asymmetries $\mathcal{A}_{\mathrm{T}}$ and $\mathcal{A}_{\mathrm{CP}}$, it is important that both these asymmetries and the cross sections $\sigma$ are large. In the following, the impact of longitudinally-polarized $e^{+}$and $e^{-}$beams of a linear collider in the $500 \mathrm{GeV}$ range on the measurement of $\mathcal{A}_{\mathrm{T}}, \mathcal{A}_{\mathrm{CP}}$ and $\sigma$ is discussed.

\section{a) Two-body decays}

The above asymmetries $\mathcal{A}_{\mathrm{T}}$ and $\mathcal{A}_{\mathrm{CP}}$ are shown in figs. 3.12-3.14 for $e^{+} e^{-} \rightarrow \tilde{\chi}_{1}^{0} \tilde{\chi}_{2}^{0}$ at $\sqrt{s}=500 \mathrm{GeV}$, with the decays (3.8) and (3.11).

In fig. 3.12 the dependence of the cross sections $\sigma\left(e^{+} e^{-} \rightarrow \tilde{\chi}_{1}^{0} \tilde{\chi}_{2}^{0}\right) \times \operatorname{BR}\left(\tilde{\chi}_{2}^{0} \rightarrow \tilde{\ell}_{R} \ell_{1}\right) \times$ $\operatorname{BR}\left(\tilde{\ell}_{R} \rightarrow \tilde{\chi}_{1}^{0} \ell_{2}\right)$ and of $\mathcal{A}_{\mathrm{T}}$ on the beam polarizations is shown $[98,109]$. The contributing CP-violating phases in that process with $\ell_{1,2} \neq \tau$ are $\varphi_{M_{1}}$ and $\varphi_{\mu}$. Since the experimental results from the EDM [79] put stringent bounds particularly on $\varphi_{\mu}$, we choose only $\varphi_{M_{1}} \neq 0$. The SUSY parameters are chosen according to scenario S8, table 3.1, and lead to $\operatorname{BR}\left(\tilde{\chi}_{2}^{0} \rightarrow \tilde{\ell}_{R} \ell_{1}\right)=0.63$ (summed over both signs of electric charge) and $\operatorname{BR}\left(\tilde{\ell}_{R} \rightarrow \tilde{\chi}_{1}^{0} \ell_{2}\right)=1$. Note that both $\mathcal{A}_{\mathrm{T}}$ and $\sigma$ are considerably enhanced for positive electron and negative positron polarizations.

$$
P_{e^{+}} \quad \sigma\left(e^{+} e^{-} \rightarrow \tilde{\chi}_{1}^{0} \tilde{\chi}_{1}^{0} \ell_{1} \ell_{2}\right) \text { in } \mathrm{fb}
$$

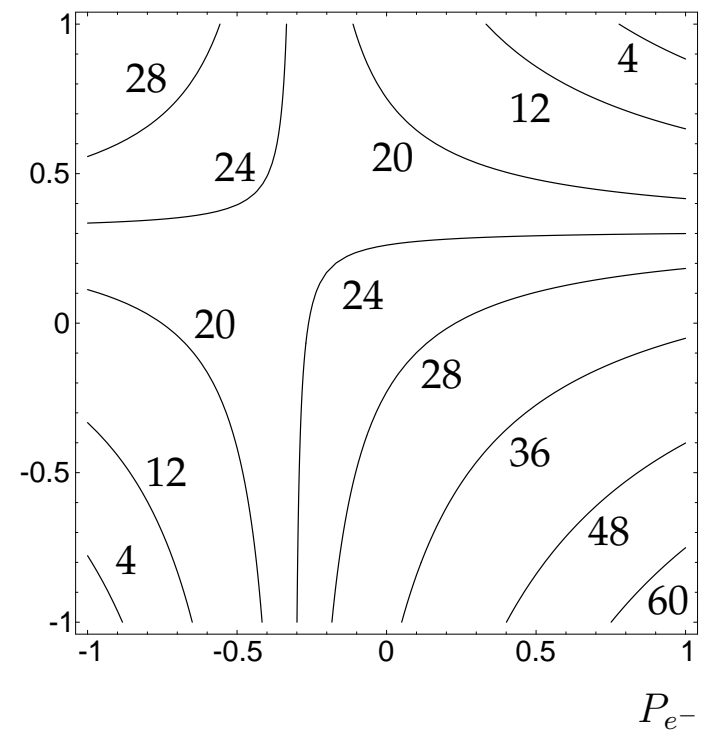

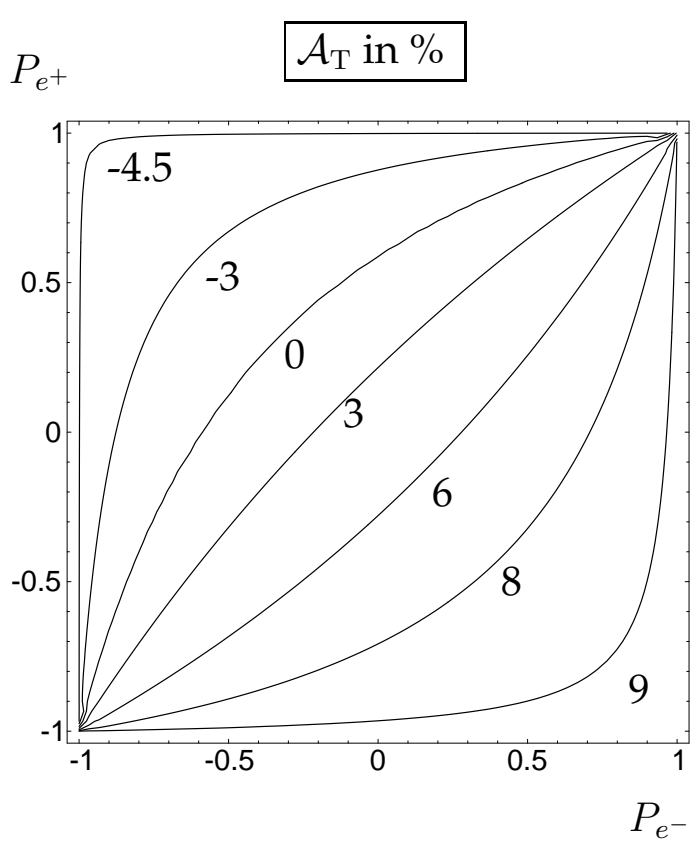

Figure 3.12: Contour lines of $\sigma$ and $\mathcal{A}_{\mathrm{T}}$ in neutralino production, $e^{+} e^{-} \rightarrow \tilde{\chi}_{1}^{0} \tilde{\chi}_{2}^{0}$ and the subsequent decay, eq. (3.8) for scenario S8, table 3.1, with the CP-violating phase $\varphi_{M_{1}}=$ $0.2 \pi$ [109].

This choice of polarization enhances the contributions of the right-slepton exchange in the neutralino production, eq. (3.7), and reduces that of the left slepton exchange $[95,110]$. 
While the contributions of right and left slepton exchanges enter $\sigma$ with the same signs, they enter $\mathcal{A}_{\mathrm{T}}$ with opposite signs, which accounts for the sign change of $\mathcal{A}_{\mathrm{T}}$ in fig. 3.12.

In figs. 3.13 and 3.14 , contour lines of the $\tau$ polarization asymmetry $\mathcal{A}_{\mathrm{CP}}$, eq. (3.12), are shown. Since in those cases the decay into $\tau \mathrm{s}$ is studied one additional CP-violating phase contributes, $\varphi_{A_{\tau}}$. Since the asymmetry $\mathcal{A}_{\mathrm{CP}}$ is very sensitive to both phases $\varphi_{A_{\tau}}$ and $\varphi_{M_{1}}$ the dependence of $\sigma=\sigma\left(e^{+} e^{-} \rightarrow \tilde{\chi}_{1}^{0} \tilde{\chi}_{2}^{0}\right) \times \operatorname{BR}\left(\tilde{\chi}_{2}^{0} \rightarrow \tilde{\tau}_{1}^{+} \tau^{-}\right)$and $\mathcal{A}_{\mathrm{CP}}$ on the beam polarization is shown in fig. 3.13 for the case $\varphi_{A_{\tau}}=\pi / 2$ in scenario S9, and in fig. 3.14 for $\varphi_{M_{1}}=0.2 \pi$ in scenario S10, see table 3.1.

In scenario S9 a large value of $\left|A_{\tau}\right|=1500 \mathrm{GeV}$ has been chosen because $\mathcal{A}_{\mathrm{CP}}$ increases with increasing $\left|A_{\tau}\right| \gg|\mu| \tan \beta[105,109]$. For unpolarized beams the asymmetry is $1 \%$. If only the electron beam is polarized, $\left(P_{e^{-}}, P_{e^{+}}\right)=(+80 \%, 0)$, the asymmetry reaches values of about $\pm 10 \%$. It achieves values larger than $\pm 13 \%$ if both beams are polarized with opposite signs. Furthermore, if both beams are polarized, one also gains in statistics. The cross section $\sigma=\sigma\left(e^{+} e^{-} \rightarrow \tilde{\chi}_{1}^{0} \tilde{\chi}_{2}^{0}\right) \times \operatorname{BR}\left(\tilde{\chi}_{2}^{0} \rightarrow \tilde{\tau}_{1}^{+} \tau^{-}\right)$, shown in fig. 3.13 with $\operatorname{BR}\left(\tilde{\chi}_{2}^{0} \rightarrow\right.$ $\left.\tilde{\tau}_{1}^{+} \tau^{-}\right)=0.22$, is very sensitive to the beam polarization and varies between $1 \mathrm{fb}$ and 30 $\mathrm{fb}$.

$$
P_{e^{+}} \sigma\left(e^{+} e^{-} \rightarrow \tilde{\chi}_{1}^{0} \tilde{\tau}_{1}^{+} \tau^{-}\right) \text {in } \mathrm{fb}
$$

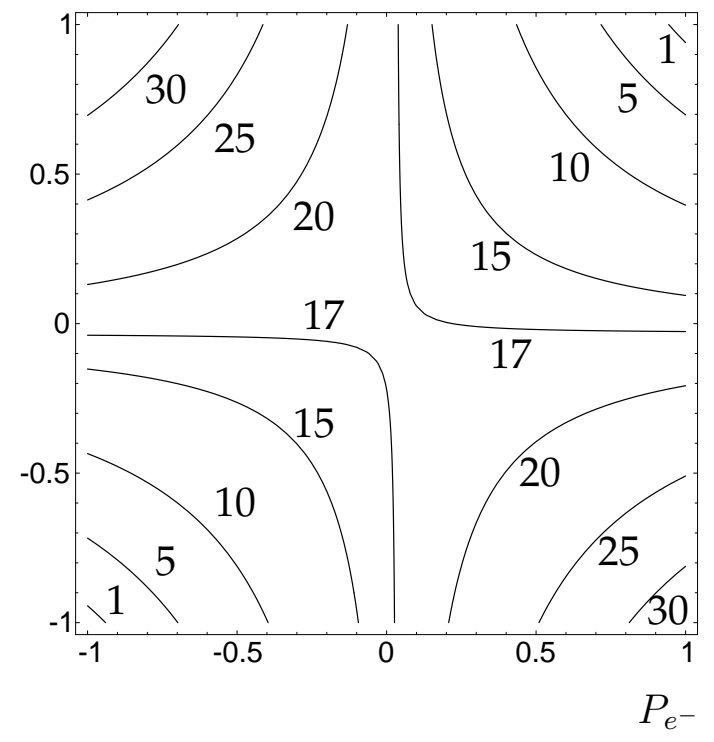

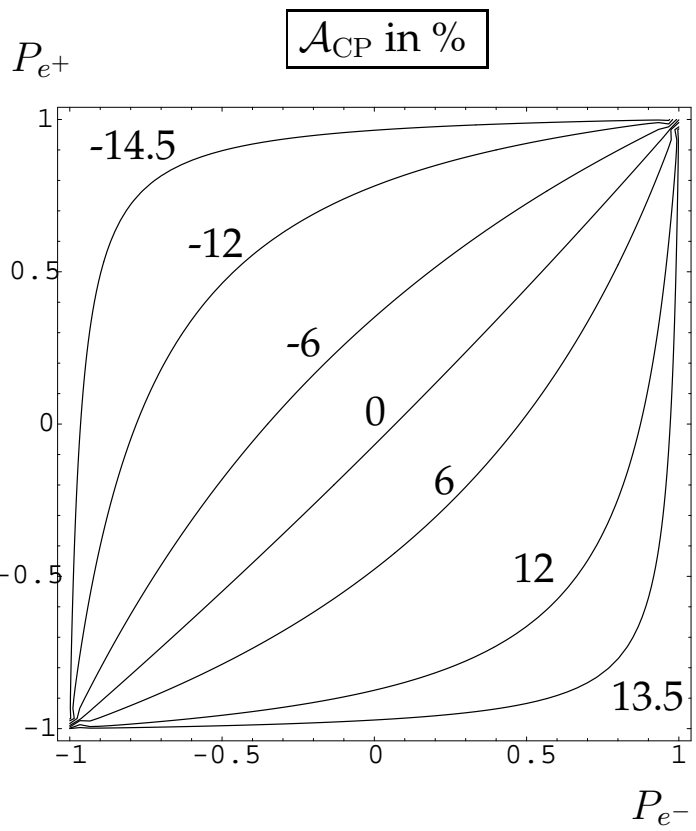

Figure 3.13: Contour lines of $\sigma$ and $\mathcal{A}_{\mathrm{CP}}$ of the process, $e^{+} e^{-} \rightarrow \tilde{\chi}_{1}^{0} \tilde{\chi}_{2}^{0}$, with subsequent decay, eq. (3.11) for the scenario S9, table 3.1, with the CP-violating phase $\varphi_{A_{\tau}}=\pi / 2$ [109].

In scenario S10 the phase $\varphi_{M_{1}} \neq 0$ is considered, which influences the neutralino $\tilde{\chi}_{1,2}^{0}$ masses and couplings. Therefore CP-violating effects occur both in the production as well as in the decay process. The neutralino branching ratio is $\operatorname{BR}\left(\tilde{\chi}_{2}^{0} \rightarrow \tilde{\tau}_{1}^{+} \tau^{-}\right)=0.19$ for the considered scenario. Despite the small phases, $\mathcal{A}_{\mathrm{CP}}$ reaches values up to $-12 \%$ for negative $e^{-}$and positive $e^{+}$beam polarizations. 

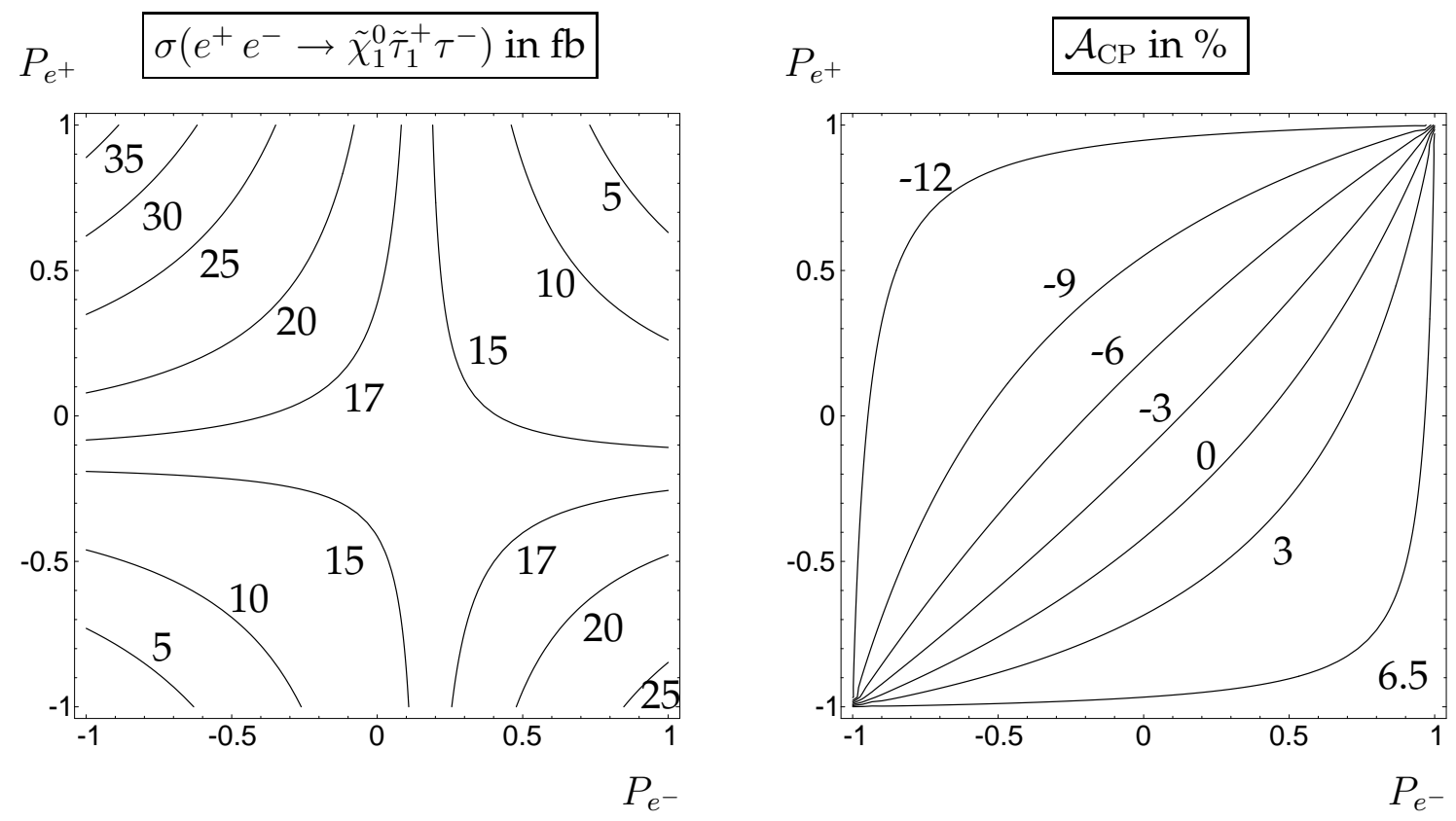

Figure 3.14: Contour lines of $\sigma$ and $\mathcal{A}_{\mathrm{CP}}$ of the process, $e^{+} e^{-} \rightarrow \tilde{\chi}_{1}^{0} \tilde{\chi}_{2}^{0}$, with subsequent decay, eq. (3.11) for the scenario S10, table 3.1, with the CP-violating phase $\varphi_{M_{1}}=0.2 \pi$ [109].

\section{b) Three-body decays}

In this subsection the influence of longitudinal $e^{-}$and $e^{+}$beam polarizations on the T-odd asymmetry $\mathcal{A}_{\mathrm{T}}$, eq. (3.10), is analysed [107]. The centre-of-mass energy is $\sqrt{s}=500 \mathrm{GeV}$ and the phases are chosen as $\varphi_{M_{1}}=0.2 \pi$ and $\varphi_{\mu}=0$.

In figs. 3.15(a) and (b) $\mathcal{A}_{\mathrm{T}}$ is shown as a function of $P_{e^{-}}$for different $e^{+}$beam polarizations, $-60 \% \leq P_{e^{+}} \leq+60 \%$. The chosen SUSY parameters, cf. S11 in table 3.1, lead to a large mixing between the gaugino and higgsino components of $\tilde{\chi}_{1}^{0}, \tilde{\chi}_{2}^{0}$ and $\tilde{\chi}_{3}^{0}$ [107]. For both production processes in fig. $3.15 \mathcal{A}_{\mathrm{T}}$ is positive (negative) for $P_{e^{-}} \rightarrow-1\left(P_{e^{-}} \rightarrow+1\right)$. For these polarizations the $\tilde{e}_{L}\left(\tilde{e}_{R}\right)$ contributions to the neutralino spin density matrix are dominant. For $\left(P_{e^{-}}, P_{e^{+}}\right)=(-90 \%, 0)$ and $(+90 \%, 0)$ the result is $\mathcal{A}_{\mathrm{T}}=+9.0 \%$ and $-6.2 \%$ for $\tilde{\chi}_{1}^{0} \tilde{\chi}_{2}^{0}$ production and $\mathcal{A}_{\mathrm{T}}=+8.3 \%$ and $-4.8 \%$ for $\tilde{\chi}_{3}^{0} \tilde{\chi}_{2}^{0}$ production, respectively. Additional positron beam polarization of opposite sign only slightly enhances these asymmetries. However, it considerably enhances the corresponding cross sections, hence the expected rates to measure $\mathcal{A}_{\mathrm{T}}$ by a factor of about 1.5, see figs. 3.15(c) and (d).

The relative statistical error on $\mathcal{A}_{\mathrm{T}}$ is given by $\delta \mathcal{A}_{\mathrm{T}}=\Delta \mathcal{A}_{\mathrm{T}} / \mathcal{A}_{\mathrm{T}}=\mathcal{N}_{\sigma} /\left(\mathcal{A}_{\mathrm{T}} \sqrt{N}\right)$ with $\mathcal{N}_{\sigma}$, the number of standard deviations and $N=\sigma \mathcal{L}_{\text {int }}$, the number of events for a total integrated luminosity $\mathcal{L}_{\text {int }}$. Assuming $\delta \mathcal{A}_{\mathrm{T}} \approx 1$ for $\mathcal{A}_{\mathrm{T}}$ to be measurable it follows that $\mathcal{N}_{\sigma} \approx\left|\mathcal{A}_{\mathrm{T}}\right| \sqrt{\sigma \mathcal{L}}$ [98]. In table 3.5, $\mathcal{A}_{\mathrm{T}}, \sigma$ and the corresponding standard deviations $\mathcal{N}_{\sigma}$ of $\mathcal{A}_{\mathrm{T}}$ are given for $\mathcal{L}_{\text {int }}=500 \mathrm{fb}^{-1}$ and several sets of beam polarizations. With $\left(\left|P_{e^{-}}\right|,\left|P_{e^{+}}\right|\right)=(90 \%, 60 \%)$ the number of standard deviations $\mathcal{N}_{\sigma}$ increases by up to a factor 1.4 for $\tilde{\chi}_{1}^{0} \tilde{\chi}_{2}^{0}$ production and by up to a factor 1.6 for $\tilde{\chi}_{3}^{0} \tilde{\chi}_{2}^{0}$ production in scenario S11, table 3.1, in comparison to only polarized electrons.

Quantitative examples: In neutralino production with subsequent two-body and three-body decays the cross sections are enhanced by a factor of about 1.5 with both 

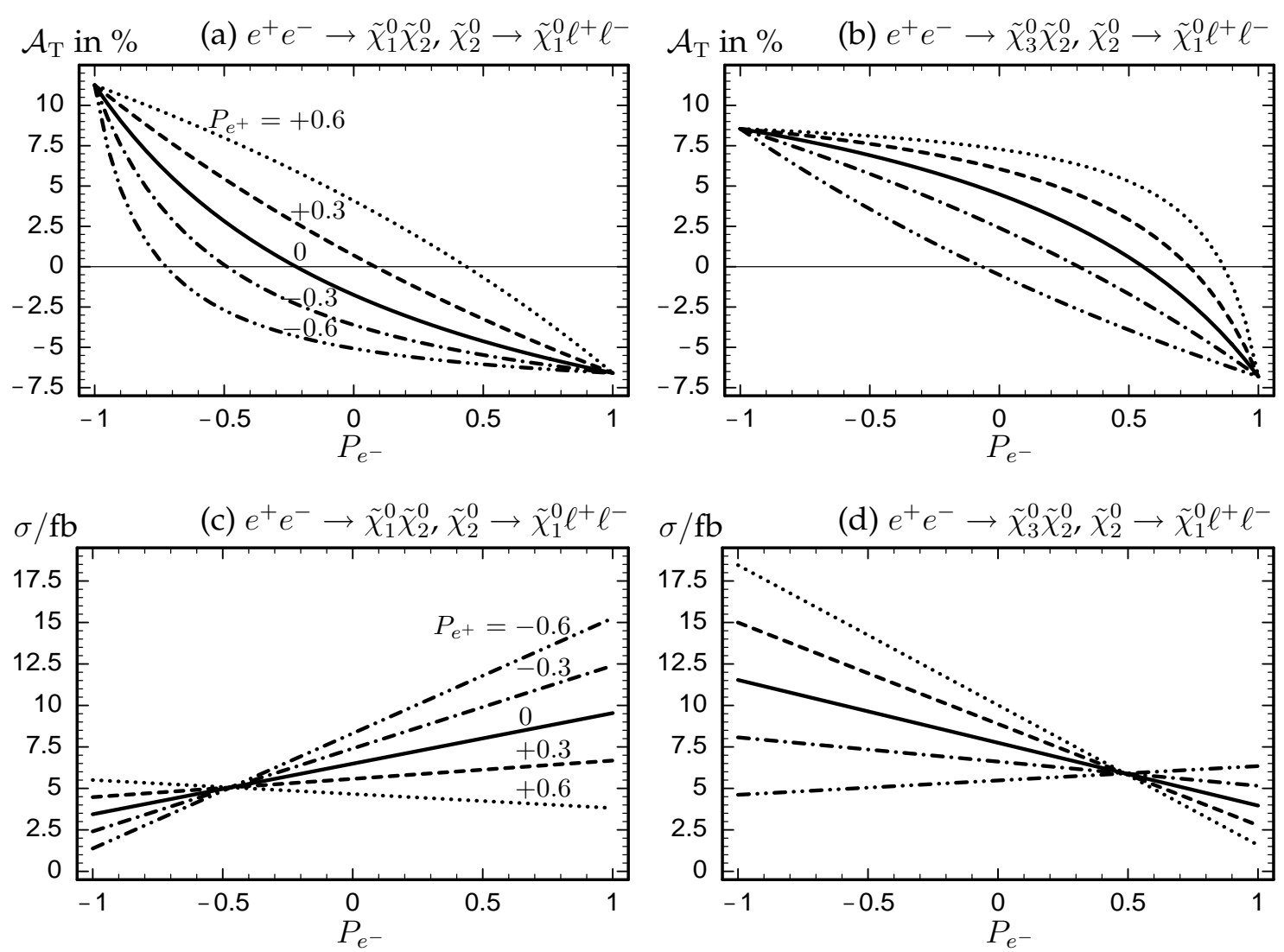

Figure 3.15: (a), (b): The T-odd asymmetry $\mathcal{A}_{\mathrm{T}}$, eq. (3.10), for $e^{+} e^{-} \rightarrow \tilde{\chi}_{i}^{0} \tilde{\chi}_{2}^{0}$, (a) $i=1$ and (b) $i=3$, with subsequent leptonic three-body decay $\tilde{\chi}_{2}^{0} \rightarrow \tilde{\chi}_{1}^{0} \ell^{+} \ell^{-}$, and (c), (d) the corresponding cross sections $\sigma=\sigma\left(e^{+} e^{-} \rightarrow \tilde{\chi}_{i}^{0} \tilde{\chi}_{2}^{0}\right) \times \operatorname{BR}\left(\tilde{\chi}_{2}^{0} \rightarrow \tilde{\chi}_{1}^{0} \ell^{+} \ell^{-}\right),(\mathrm{c}) i=1$ and (d) $i=3$, summed over $\ell=e, \mu$, as a function of the $e^{-}$beam polarization $P_{e^{-}}$for different $e^{+}$ beam polarizations $P_{e^{+}}$and $\sqrt{s}=500 \mathrm{GeV}$ in the scenario S11, table 3.1 [107].

\begin{tabular}{|l||c|c|c|c|c||c|c|c|c|c|}
\hline \multicolumn{1}{|c||}{} & \multicolumn{3}{c||}{$e^{+} e^{-} \rightarrow \tilde{\chi}_{1}^{0} \tilde{\chi}_{2}^{0}, \tilde{\chi}_{2}^{0} \rightarrow \tilde{\chi}_{1}^{0} \ell^{+} \ell^{-}$} & \multicolumn{5}{c|}{$e^{+} e^{-} \rightarrow \tilde{\chi}_{3}^{0} \tilde{\chi}_{2}^{0}, \tilde{\chi}_{2}^{0} \rightarrow \tilde{\chi}_{1}^{0} \ell^{+} \ell^{-}$} \\
\hline$\left(P_{e^{-}}, P_{e^{+}}\right)$ & $(0,0)$ & $(-, 0)$ & $(-,+)$ & $(+, 0)$ & $(+,-)$ & $(0,0)$ & $(-, 0)$ & $(-,+)$ & $(+, 0)$ & $(+,-)$ \\
\hline $\mathcal{A}_{\mathrm{T}}[\%]$ & -1.8 & 9.0 & 10.6 & -6.2 & -6.5 & 4.5 & 8.3 & 8.5 & -4.8 & -6.3 \\
$\sigma[\mathrm{fb}]$ & 6.5 & 3.7 & 5.4 & 9.2 & 14.6 & 7.8 & 11.2 & 17.6 & 4.3 & 6.3 \\
$\mathcal{N}_{\sigma}$ & 1.0 & 3.9 & 5.5 & 4.2 & 5.6 & 2.8 & 6.2 & 8.0 & 2.2 & 3.5 \\
\hline
\end{tabular}

Table 3.5: The T-odd asymmetries $\mathcal{A}_{\mathrm{T}}$, eq. (3.10), are listed for $e^{+} e^{-} \rightarrow \tilde{\chi}_{i}^{0} \tilde{\chi}_{2}^{0}, i=1,3$, with subsequent leptonic three-body decay $\tilde{\chi}_{2}^{0} \rightarrow \tilde{\chi}_{1}^{0} \ell^{+} \ell^{-}$. The corresponding cross section $\sigma=\sigma\left(e^{+} e^{-} \rightarrow \tilde{\chi}_{i}^{0} \tilde{\chi}_{2}^{0}\right) \times \operatorname{BR}\left(\tilde{\chi}_{2}^{0} \rightarrow \tilde{\chi}_{1}^{0} \ell^{+} \ell^{-}\right)$, summed over $\ell=e, \mu$, and the standard deviations $\mathcal{N}_{\sigma}=\left|\mathcal{A}_{\mathrm{T}}\right| \sqrt{\sigma \mathcal{L}_{\text {int }}}$ are given for $\mathcal{L}_{\text {int }}=500 \mathrm{fb}^{-1}, \sqrt{s}=500 \mathrm{GeV}$ and several beam polarizations $\left(P_{e^{-}}, P_{e^{+}}\right)=( \pm 90 \%, \pm 60 \%)$ in the scenario S11, table 3.1 [107].

beams polarized compared with only polarized electrons. The CP-odd and T-odd asymmetries are enhanced by up to a factor of $\mathbf{1 . 3}$ and their measurability by up to a factor of about 1.6 with both beams polarized compared with the case of only polarized electrons.

\subsubsection{SUSY CP asymmetries with transversely-polarized beams}

Only if both beams are polarized, it is possible to exploit transversely-polarized $e^{-}$ 
and $e^{+}$beams in chargino/neutralino production and decay. Both CP-even and CP-odd azimuthal asymmetries can be constructed which give access to determine $\mathrm{CP}$-violating phases in a broad range of the MSSM parameter space.

Transversely-polarized beams offer the possibility of detailed studies of the effects of $\mathrm{CP}$ violation. They give access to azimuthal asymmetries that can be defined directly in terms of products of final particle momenta, without the need to measure final-state polarizations. For $(V, A)$ interactions, due to the negligible electron mass, new observables involving transversely-polarized beams are available only if both beams are polarized. In this section the effects of transversely-polarized beams in the chargino [111] and neutralino [112] processes

$$
\begin{array}{ll}
e^{+} e^{-} \rightarrow \tilde{\chi}_{1}^{+} \tilde{\chi}_{j}^{-}, \quad \text { with } \quad \tilde{\chi}_{j}^{-} \rightarrow \tilde{\nu}_{\ell} \ell^{-} \quad \text { and } \quad \tilde{\chi}_{j}^{-} \rightarrow W^{-} \tilde{\chi}_{1}^{0}, \quad j=1,2, \\
e^{+} e^{-} \rightarrow \tilde{\chi}_{1}^{0} \tilde{\chi}_{j}^{0}, \quad \text { with } \quad \tilde{\chi}_{j}^{0} \rightarrow \tilde{\ell}_{L, R}^{ \pm} \ell_{1}^{\mp} \rightarrow \ell_{1}^{\mp} \ell_{2}^{ \pm} \tilde{\chi}_{1}^{0}, \quad j=2,3,4,
\end{array}
$$

are summarised.

With transversely-polarized beams new CP-sensitive observables such as CP-odd triple product correlations as well as CP-even azimuthal asymmetries can be exploited. $\mathrm{CP}$-odd observables are necessary to determine the underlying interactions unambiguously. Only two complex parameters enter the neutralino/chargino sector: the higgsino mass parameter $\mu$ and the gaugino mass parameter $M_{1}$.

\section{Transverse beam polarization in chargino production}

In the case of chargino production and decay, eq. (3.13), T-odd asymmetries based on triple products of the electron/positron momentum, transverse polarization vector and the momentum of the outgoing lepton or $W$ vanish [111]. Therefore, T-odd observables could only be constructed in that case by analyzing both decays of $\tilde{\chi}_{1}^{ \pm}$and of $\tilde{\chi}_{2}^{\mp}$, taking into account the spin-spin correlations between both decaying charginos [111].

On the other hand, transverse polarizations allow to construct CP-even azimuthal asymmetries $[113,114]$, which are sensitive to the phases $\varphi_{M_{1}}$ and $\varphi_{\mu}$ :

$$
A_{\phi}=\frac{1}{\sigma}\left[\int_{+} \frac{\mathrm{d} \sigma}{\mathrm{d} \phi} \mathrm{d} \phi-\int_{-} \frac{\mathrm{d} \sigma}{\mathrm{d} \phi} \mathrm{d} \phi\right],
$$

where $\phi$ is the azimuthal angle of the observed final $\ell$ or $W^{-}$, eq. (3.13), $\int_{ \pm}$refers to the integrated phase space hemisphere above (below) the scattering plane and $\mathrm{d} \sigma / \mathrm{d} \phi$ denotes the differential cross section for production and decay including the complete spin correlations of the charginos. It has been shown for different scenarios in [111] and for the process (3.13) that this CP-even asymmetry is a promising observable for the determination of the phase $\varphi_{M_{1}}$ (up to a two-fold ambiguity) and can reach up to about $20 \%$.

\section{Transverse beam polarization in neutralino production}

Contrary to the case of chargino production (Dirac fermions), it is possible to construct T-odd triple product correlations in the production of neutralinos (Majorana fermions) [112].

Consider first CP-odd observables in neutralino production with transverse $e^{ \pm}$beam polarization, eq. (3.14). The $e^{-}$direction is chosen as the $z$ axis and the direction of its 
transverse polarization vector as the $x$ axis. If the angle between the transverse polarization vectors of the $e^{+}$and the $e^{-}$is $\pi / 2$, with $\phi_{+}=\pi / 2$ and $\phi_{-}=0$ ), the CP-violating contributions can be isolated with the following integration (this $A_{\mathrm{CP}}(\theta)$ should not be confused with the $\mathcal{A}_{\mathrm{CP}}$ of eq. (3.12)):

$$
A_{\mathrm{CP}}(\theta)=\frac{1}{\sigma}\left[\int_{0}^{\pi / 4}-\int_{\pi / 4}^{3 \pi / 4}+\int_{3 \pi / 4}^{5 \pi / 4}-\int_{5 \pi / 4}^{7 \pi / 4}+\int_{7 \pi / 4}^{2 \pi}\right] \frac{\mathrm{d}^{2} \sigma}{\mathrm{d} \phi \mathrm{d} \cos \theta} \mathrm{d} \phi .
$$

Because of the Majorana nature of the neutralinos the CP-violating terms of the $t$ - and $u$ channel contributions cancel each other, if integrated over the whole range of $\theta$. Therefore, the CP-odd asymmetry is defined as

$$
A_{\mathrm{CP}}=\left[\int_{0}^{\pi / 2}-\int_{\pi / 2}^{\pi}\right] A_{\mathrm{CP}}(\theta) \mathrm{d} \cos \theta .
$$

In order to measure $A_{\mathrm{CP}}$ it is necessary to reconstruct the directions of the neutralinos, this can be done by analysing the subsequent two-body decays in eq. (3.14).

Fig. 3.16a shows contours of $A_{C P}$ in $e^{+} e^{-} \rightarrow \tilde{\chi}_{1}^{0} \tilde{\chi}_{2}^{0}$ at $\sqrt{s}=500 \mathrm{GeV}$ in the $|\mu|-M_{2}$ SUSY parameter plane with the other MSSM parameters as given in S12, table 3.1. The CP-odd asymmetry $A_{\mathrm{CP}}$, eq. (3.17), is sizable for large mixing between the gaugino and the higgsino components of $\tilde{\chi}_{1}^{0}$ and $\tilde{\chi}_{2}^{0}$. If the beams were fully transversely polarized, $\left(P_{e^{-}}^{\mathrm{T}}, P_{e^{+}}^{\mathrm{T}}\right)=(100 \%, 100 \%)$, then $A_{C P}$ could reach up to about $8.8 \%$.

Since the asymmetries are here only of the order of a few percent, it is important to know how much luminosity $\mathcal{L}_{\text {int }}$ is required for a measurement with sufficient significance. It can be estimated from the relation [98,112]

$$
\mathcal{L}_{\text {int }}=\left(\mathcal{N}_{\sigma}\right)^{2} /\left[\left(P_{e^{-}}^{\mathrm{T}} P_{e^{+}}^{\mathrm{T}} A_{\mathrm{CP}}\right)^{2} \sigma\right],
$$

where $\mathcal{N}_{\sigma}$ denotes the number of standard deviations and $\sigma$ the corresponding neutralino cross section. In fig. $3.16 \mathrm{~b}$ is shown how much luminosity is required with $\left(P_{e^{-}}^{\mathrm{T}}, P_{e^{+}}^{\mathrm{T}}\right)=$ $(80 \%, 60 \%)$ for a discovery with $5-\sigma$. For the maximum value of the asymmetry $A_{\mathrm{CP}}$ the required luminosity $\mathcal{L}_{\text {int }}$ is about $80 \mathrm{fb}^{-1}$.

The reconstruction of the direction of the neutralinos is not necessary if a CP-odd asymmetry is defined with respect to the azimuth of the final leptons $\ell_{1,2}$ [112]. The derived asymmetries are of the same order of magnitude as in the case studied before. At a LC an arbitrary and independent relative orientation of both transversely-polarized beams could be studied which has not received much attention in the literature so far and provides high flexibility and a larger amount of observables [112].

Quantitative examples: With transversely-polarized $e^{-}$and $e^{+}$beams new CPsensitive observables could be observed. In chargino production and decay $\mathrm{CP}$-even azimuthal asymmetries of the order of $10 \%$ are obtained. In neutralino production CP-odd as well as CP-even asymmetries can be constructed. At $\sqrt{s}=500 \mathrm{GeV}$ with $\left(P_{e^{-}}^{\mathrm{T}}, P_{e^{+}}^{\mathrm{T}}\right)=(80 \%, 60 \%)$ and an integrated luminosity $\mathcal{L}_{\mathrm{int}}=500 \mathrm{fb}^{-1}$, the discovery of a CP-odd asymmetry $A_{C P}$ at the 5- $\sigma$ level is possible for a broad range of parameters. 

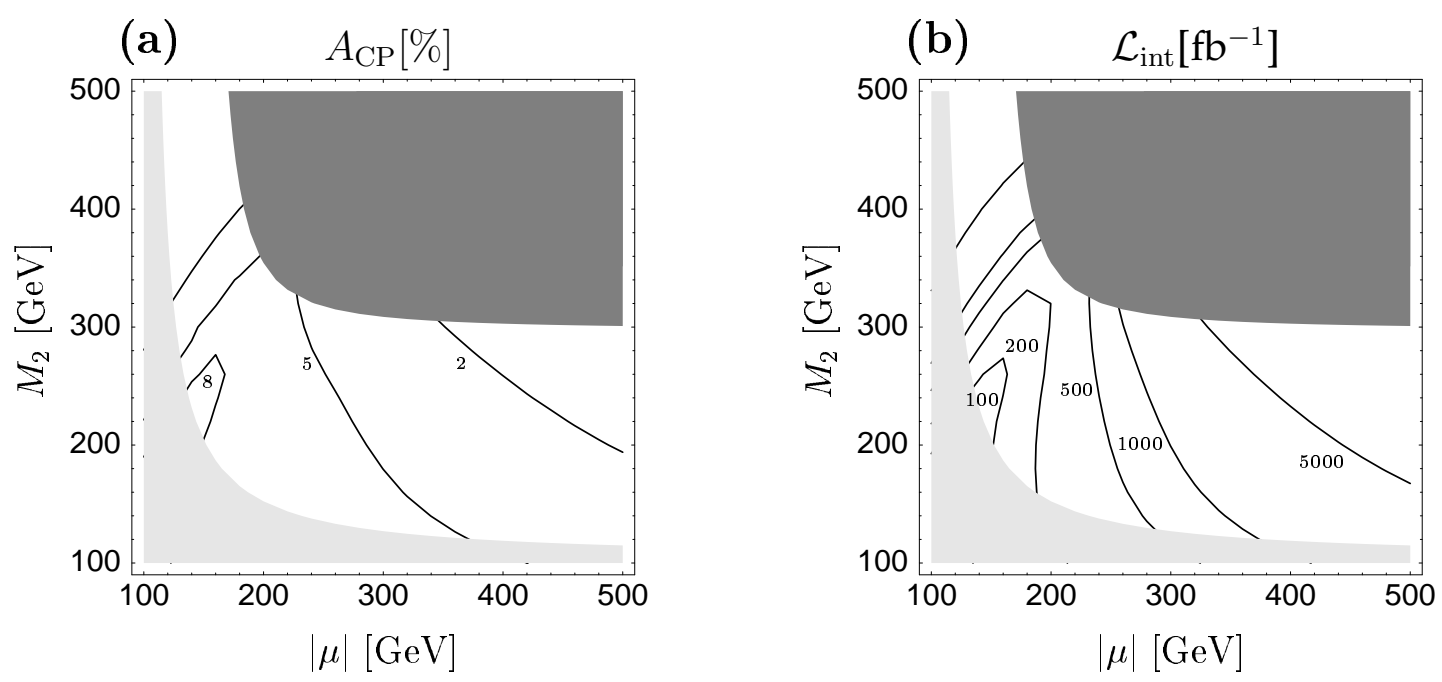

Figure 3.16: Contours of (a) the CP-odd asymmetry $A_{\mathrm{CP}}$, Eq. (3.17), for neutralino production, $e^{+} e^{-} \rightarrow \tilde{\chi}_{1}^{0} \tilde{\chi}_{2}^{0}$, at $\sqrt{s}=500 \mathrm{GeV}$ with transverse beam polarization $\left(P_{e^{-}}^{\mathrm{T}}, P_{e^{+}}^{\mathrm{T}}\right)=$ $(100 \%, 100 \%)$ in the $|\mu|-M_{2}$ plane for the scenario S12, table 3.1, and (b) the luminosity $\mathcal{L}_{\text {int }}$ needed to measure the asymmetry at the 5- $\sigma$ level with a degree of transverse polarization $\left(P_{e^{-}}^{\mathrm{T}}, P_{e^{+}}^{\mathrm{T}}\right)=(80 \%, 60 \%)$. The light gray region is experimentally excluded because $m_{\tilde{\chi}_{1}^{ \pm}}$has to be larger then the exclusion bound $104 \mathrm{GeV}$ (LEP limit). The dark gray region is excluded because $m_{\tilde{\chi}_{1}^{0}}$ has to be smaller than $m_{\tilde{e}_{R}}$ (since $m_{\tilde{\chi}_{1}^{0}}$ is assumed to be the stable LSP) [112].

\subsubsection{Non-minimal SUSY models}

\subsubsection{Extended neutralino sector}

Extensions of the MSSM particle sector, e.g. by adding Higgs singlets or new gauge bosons, enlarge the neutralino sector. Cross sections for the production of singlinodominated neutralinos are typically suppressed. The polarization of both beams may be crucial for a) observing signals with production of singlino-dominated neutralinos and b) distinguishing between the MSSM and the extended model. Furthermore the light neutralino cross sections may show opposite dependences on polarization, so that a model distinction becomes possible.

Non-minimal extensions of the particle sector of the MSSM are characterized by an additional singlet superfield $S$ with vacuum expectation value $x$ and a trilinear coupling $\lambda$ relating the singlet superfield and the two doublet Higgs superfields in the superpotential. In the Next-to-Minimal Supersymmetric Standard Model (NMSSM) [115-118, 120, 121], the superpotential contains also a trilinear term of the singlet superfield with coupling $\kappa$,

$$
W \subset \lambda H_{1} H_{2} S+\frac{1}{3} \kappa S^{3} .
$$

In $\mathrm{E}_{6}$-inspired models [122] one has in addition to the singlet superfield one extra neutral gauge boson $Z^{\prime}$. There is an additional gaugino mass parameter $M^{\prime}$ related to this extra $\mathrm{U}(1)$ gauge factor.

Dominant singlet higgsino (singlino) component in the lightest neutralino exist for large vacuum expectation values $x \gtrsim 1 \mathrm{TeV}$ [123]. 


\section{Production of singlino-dominated neutralinos}

Since the singlino component does not couple to gauge bosons, gauginos, (scalar) leptons and (scalar) quarks, cross sections for the production of the exotic neutralinos are generally small [124-127]. However, they may be produced at a high luminosity $e^{+} e^{-}$linear collider with cross sections sufficient for detection, which can still be enhanced by using the polarization of both beams. For a wide range of $x$ values the associated production of the singlino-dominated neutralino yields detectable cross sections. In the NMSSM [115] a singlino-dominated neutralino $\tilde{\chi}_{S}^{0}$ with mass $\approx 2 \kappa x$ decouples from the other neutralinos for large $x \gg\left|M_{2}\right|$ in the neutralino mixing matrix, while the other neutralinos $\tilde{\chi}_{1, \ldots, 4}^{0}$ have MSSM character. In an $\mathrm{E}_{6}$-inspired model with one extra neutral gauge boson $Z^{\prime}$ and one additional singlet superfield, that contains six neutralinos, a nearly pure light singlino-like $\tilde{\chi}_{S}^{0}$ exists for very large values $\left|M^{\prime}\right| \gg x$ with mass $\approx 0.18 x^{2} /\left|M^{\prime}\right|$ in zeroth approximation [123, 124,128].

In fig. 3.17 the cross section for associated production of the singlino-dominated $\tilde{\chi}_{S}^{0}$ together with the lightest MSSM-like neutralino $\tilde{\chi}_{1}^{0}$ is shown. The scenarios considered are such that the MSSM-like neutralinos have similar masses and mixing character as in the 'typical mSUGRA' scenario S6, cf. table 3.1, with a chosen $\mu_{\text {eff }}=\lambda x=352 \mathrm{GeV}$. The resulting neutralino masses are $96,177,359$ and $378 \mathrm{GeV}$. The cross sections are shown for unpolarized beams and beam polarizations $\left(P_{e^{-}}, P_{e^{+}}\right)=(+80 \%, 0)$ and $(+80 \%,-60 \%)$. Electron beam polarization $P_{e^{-}}=+80 \%$ enhances the cross section by a factor 1.5 to 1.8 , while additional positron beam polarization $P_{e^{+}}=-60 \%$ gives a further enhancement factor of about 1.6. Assuming a cross section of $1 \mathrm{fb}$ to be sufficient for discovery, the singlino-dominated neutralino can be detected with unpolarized beams for $x<7.4 \mathrm{TeV}$ $(9.7 \mathrm{TeV})$ in the NMSSM with $m_{\tilde{\chi}_{S}^{0}}=70 \mathrm{GeV}(120 \mathrm{GeV})$ and for $x<8.5 \mathrm{TeV}(6.4 \mathrm{TeV})$ in the $\mathrm{E}_{6}$ model. For a polarized electron beam, the reach in $x$ is enhanced to $x<10.0 \mathrm{TeV}$ $(12.3 \mathrm{TeV})$ in the NMSSM and $x<11.4 \mathrm{TeV}(7.9 \mathrm{TeV})$ in the $\mathrm{E}_{6}$ model, and for both beams polarized to $x<12.6 \mathrm{TeV}(15.5 \mathrm{TeV})$ in the NMSSM and $x<14.4 \mathrm{TeV}(10.0 \mathrm{TeV})$ in the $\mathrm{E}_{6}$ model; see table 3.6.

With both beams polarized the cross sections are enhanced by a factor 2.4-2.9 with respect to unpolarized beams, depending on the scenario. This enhances the reach for the singlino-dominated neutralinos to singlet vacuum expectation values as large as $15 \mathrm{TeV}$.

\begin{tabular}{|c||c|c||c|c|}
\multicolumn{5}{|c|}{$x$ reach $[\mathrm{TeV}]$} \\
$\left(P_{e^{-}}, P_{e^{+}}\right)$ & $m_{\tilde{\chi}_{S}^{0}}=70 \mathrm{GeV}$ & $m_{\tilde{\chi}_{S}^{0}}=120 \mathrm{GeV}$ & $m_{\tilde{\chi}_{S}^{0}}=70 \mathrm{GeV}$ & $m_{\tilde{\chi}_{S}^{0}}=120 \mathrm{GeV}$ \\
\hline$(0,0)$ & 7.4 & 9.7 & 8.5 & 6.4 \\
$(+80 \%, 0)$ & 10.0 & 12.3 & 11.4 & 7.9 \\
$(+80 \%,-60 \%)$ & 12.6 & 15.5 & 14.4 & 10.0 \\
\hline
\end{tabular}

Table 3.6: Accessible range of the singlet vacuum expectation value $x$ under the discovery assumption of $\sigma\left(e^{+} e^{-} \rightarrow \tilde{\chi}_{S}^{0} \tilde{\chi}_{i}^{0}\right) \geq 1 \mathrm{fb}$ [124]. The SUSY parameters are chosen corresponding to scenario S6 with $\mu_{\text {eff }}=\lambda x=352 \mathrm{GeV}$, cf table 3.1. The mass of the $\tilde{\chi}_{S}^{0}$ is fixed at $70 \mathrm{GeV}$ and $120 \mathrm{GeV}$ by the parameters $\kappa$ (NMSSM) and $M^{\prime}$ (E $\mathrm{E}_{6}$ model) [124]. 



Figure 3.17: Cross sections for the production of a singlino-dominated neutralino $\tilde{\chi}_{S}^{0}$ via $e^{+} e^{-} \rightarrow \tilde{\chi}_{S}^{0} \tilde{\chi}_{1}^{0}$ at $\sqrt{s}=500 \mathrm{GeV}$, for unpolarized beams (solid) and beam polarizations of $\left(P_{e^{-}}, P_{e^{+}}\right)=(+80 \%, 0)$ (dotted) and $(+80 \%,-60 \%)$ (dashed), in the NMSSM and an $\mathrm{E}_{6}$-inspired model. The SUSY parameters are chosen corresponding to scenario S6 with $\mu_{\text {eff }}=\lambda x=352 \mathrm{GeV}$, cf table 3.1. The mass of the $\tilde{\chi}_{S}^{0}$ is fixed at $70 \mathrm{GeV}$ and $120 \mathrm{GeV}$ by the parameters $\kappa$ (NMSSM) and $M^{\prime}$ ( $\mathrm{E}_{6}$ model) [124].

\section{Distinction between MSSM and NMSSM}

There are regions of the SUSY parameter space where the distinction between the MSSM and the NMSSM is very difficult (see for instance [129] and references therein). If the whole neutralino sector is kinematically accessible, sum rules for the production cross sections show a different energy dependence in the MSSM and the NMSSM [94]. If, however, only a part of the spectrum is accessible, polarization effects play an important role. As an example, consider the case where only the two lightest neutralinos are accessible at $\sqrt{s}=500 \mathrm{GeV}$ with, however, very low cross sections in the MSSM as well as in the NMSSM. Only with polarized beams the cross sections are sufficiently enhanced to become measurable. Furthermore, the different dependence on polarization allows a distinction between the models, as exemplified in table 3.7. The cross sections for both beams polarized are enhanced by a factor of 1.6 with respect to the case of only electrons polarized. 


\begin{tabular}{|c|c|cc|cc|}
\hline \multicolumn{7}{|c|}{$\sigma\left(\tilde{\chi}_{1}^{0} \tilde{\chi}_{2}^{0}\right)[\mathrm{fb}]$} \\
\hline MSSM & Unpolarized & $(-90 \%, 0)$ & $(+90 \%, 0)$ & $(-90 \%,+60 \%)$ & $(+90 \%,-60 \%)$ \\
NMSSM & 0.6 & 0.8 & 0.5 & 1.3 & 0.7 \\
\hline
\end{tabular}

Table 3.7: Cross section for the process $e^{+} e^{-} \rightarrow \tilde{\chi}_{1}^{0} \tilde{\chi}_{2}^{0}$ at $\sqrt{s}=500 \mathrm{GeV}$ for beam polarization configuration $\left(P_{e^{-}}, P_{e^{+}}\right)$. The light-neutralino masses are given by $m_{\tilde{\chi}_{1}^{0}}=189 \mathrm{GeV}$ and $m_{\tilde{\chi}_{2}^{0}}=267 \mathrm{GeV}$ for the scenario S13 a) (MSSM parameters) and S13 b) (NMSSM parameters), cf. table 3.1. The heavy neutralinos, the sleptons and the light chargino are assumed to be kinematically inaccessible [126].

Quantitative examples: In extensions of the MSSM including an additional Higgs singlet or a new gauge boson, the polarization of both beams enhances the rates of the neutralino cross sections by about a factor 1.6, as well as the reach on the singlet vacuum expectation value by about a factor 1.3 , up to about $15 \mathrm{TeV}$, with respect to the case where only electrons are polarized.

\subsubsection{R-parity-violating SUSY}

If R-parity is not conserved, scalar neutral particles, such as sneutrinos, can be produced in the $s$-channel giving rise to spectacular signals. With left-polarized electrons and left-polarized positrons, the signal uniquely indicates the production of a spinzero particle.

The assumption that R-parity is conserved has no strong theoretical justification. Allowing R-parity-violation, in general, one has to introduce in the superpotential the interaction terms that violate lepton and/or baryon number. For illustration purposes we restrict our discussion to the trilinear lepton number violating terms [130]:

$$
W_{R_{P}}=\lambda_{i j k} L_{L}^{i} L_{L}^{j} \bar{E}_{R}^{k}+\lambda_{i j k}^{\prime} L_{L}^{i} Q_{L}^{j} \bar{D}_{R}^{k}
$$

where $L_{L}\left(Q_{L}\right)$ denotes the left-handed doublets of leptons (quarks) and $E_{R}\left(D_{R}\right)$ the right-handed singlets of the charged leptons (down-type quarks), and $i, j, k$ stand for generation numbers. Respecting the current experimental bounds for the couplings [131], the following SUSY processes, that receive the $s$-channel $\tilde{\nu}_{\tau}$ exchange,

$$
\begin{gathered}
e^{+} e^{-} \rightarrow \tilde{\nu}_{\tau} \rightarrow e^{+} e^{-} \\
e^{+} e^{-} \rightarrow \tilde{\nu}_{\tau} \rightarrow \mu^{+} \mu^{-},
\end{gathered}
$$

are possible, could be observed at the ILC. The contributing diagrams are shown in figs. 3.18 and 3.19 , cf. also [132]. The striking effects of the spin-0 s-channel exchange can be enhanced using the LL configuration of beam polarization. The sneutrino production channel in eq. (3.21) receives a strong background from Bhabha scattering ( $s$ - and $t$-channel $\gamma / Z$ exchange), as well as also from the $t$-channel sneutrino exchange. Therefore the spin- 0 verification with beam polarization may be weakened for this process compared to the process in eq. (3.22). The situation is illustrated in fig. 3.20 for the Rparity-violating couplings $\lambda_{131}=0.05, \lambda_{232}=0.05$, scenario S14 in table 3.1. The role of 

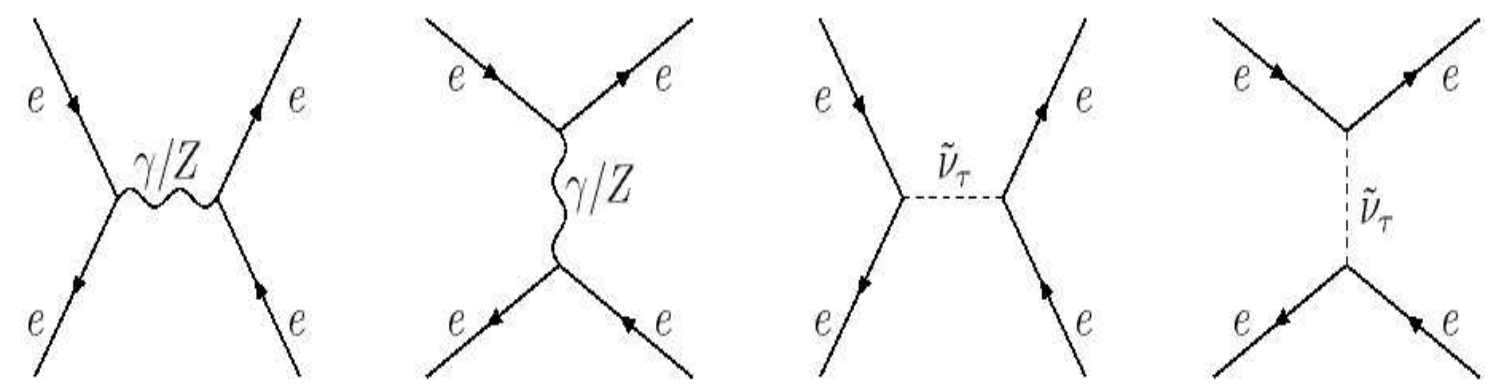

Figure 3.18: Diagrams for Bhabha scattering $e^{+} e^{-} \rightarrow e^{+} e^{-}$including $s$ - and $t$-channel exchange of $\tilde{\nu}_{\tau}\left(\lambda_{131} \neq 0\right)$.
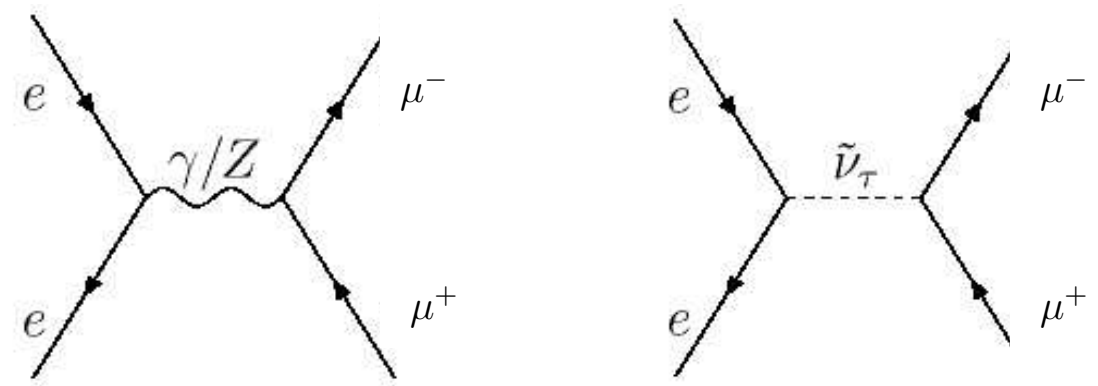

Figure 3.19: Diagrams for muon production $e^{+} e^{-} \rightarrow \mu^{+} \mu^{-}$with $s$-channel exchange of $\tilde{\nu}_{\tau}$ $\left(\lambda_{131} \neq 0, \lambda_{232} \neq 0\right)$.

the same-sign initial beam polarization in enhancing the signal over the background is evident, (cf. also table 3.8 [133]).

For the same final states, the $s$-channel $Z^{\prime}$ exchange with $m_{Z^{\prime}} \sim m_{\tilde{\nu}}$ would manifest itself by a similar peak in the cross section if the beams were RL or LR polarized since this configuration would be preferred in that case [135]. Therefore, beam polarization of both beams provides an alternative approach for verifying the spin of the exchanged objects and distinguishing the models without final-state analysis.

In the above example both the production and decay of $\tilde{\nu}_{\tau}$ violates R-parity. However, sneutrino can decay via R-parity conserving coupling, which may be the dominant decay mode. In this case we get another interesting class of R-parity violating processes in which single SUSY particle production can occur [136]. For example, single chargino (neutralino) production,

$$
e^{+} e^{-} \rightarrow \tilde{\chi}_{1}^{ \pm} \mu^{\mp}
$$

occurs via sneutrino (charged slepton) exchange in the $s$ - and $t$-channels. The chargino $\tilde{\chi}_{1}^{ \pm}$decays subsequently into $\ell^{ \pm} \tilde{\chi}_{1}^{0} \nu$ and $\tilde{\chi}_{1}^{0}$ into 3 leptons. The process requires the same lepton flavour violating couplings as in the example shown above. The characteristic feature of this process is that it requires same sign helicities, the LL configuration.

Quantitative example: Electron polarization with $P_{e^{-}}=-80 \%$ enhances the signal, i.e. Bhabha scattering or muon production including scalar neutrino exchange in the $s$-channel, only slightly by about $2-3 \%$, whereas the simultaneous polarization 

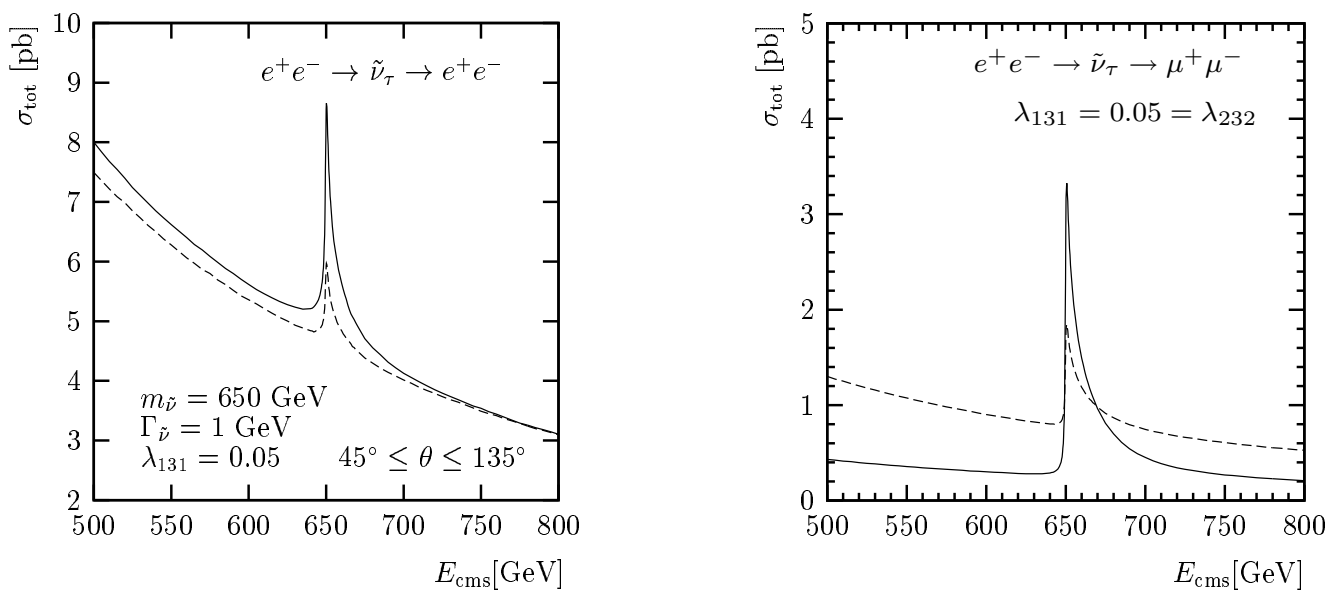

Figure 3.20: Sneutrino production in the R-parity-violating model. Resonance production $e^{+} e^{-} \rightarrow \tilde{\nu}_{\tau}$ interfering with Bhabha scattering (left panel) and resonance production for $e^{+} e^{-} \rightarrow \tilde{\nu}_{\tau} \rightarrow \mu^{+} \mu^{-}$(right panel) for different configurations of beam polarization: $\left(P_{e^{-}}, P_{e^{+}}\right)=(-80 \%,+60 \%)$ (dashed), $(-80 \%,-60 \%)$ (solid). An angular cut of $45^{\circ} \leq \theta \leq 135^{\circ}$ has been applied in Bhabha scattering [134].

Cross section $\sigma[\mathrm{pb}]$

\begin{tabular}{|c||c|c||c|c|}
\hline$\left(P_{e^{-}}, P_{e^{+}}\right)$ & $\begin{array}{c}e^{+} e^{-} \rightarrow e^{+} e^{-} \text {with } \\
e^{+} e^{-} \rightarrow \tilde{\nu}_{\tau} \rightarrow e^{+} e^{-}\end{array}$ & Bhabha & $\begin{array}{c}e^{+} e^{-} \rightarrow \mu^{+} \mu^{-} \text {with } \\
e^{+} e^{-} \rightarrow \tilde{\nu}_{\tau} \rightarrow \mu^{+} \mu^{-}\end{array}$ & $e^{+} e^{-} \rightarrow \mu^{+} \mu^{-}$ \\
\hline unpolarized & 7.17 & 4.50 & 2.50 & 0.44 \\
$(-80 \%, 0)$ & 7.32 & 4.63 & 2.58 & 0.52 \\
$(-80 \%,-60 \%)$ & 8.66 & 4.69 & 3.33 & 0.28 \\
$(-80 \%,+60 \%)$ & 5.97 & 4.58 & 1.85 & 0.78 \\
\hline
\end{tabular}

Table 3.8: Cross sections for sneutrino production in $e^{+} e^{-} \rightarrow \tilde{\nu}_{\tau} \rightarrow e^{+} e^{-}$and $e^{+} e^{-} \rightarrow$ $\tilde{\nu}_{\tau} \rightarrow \mu^{+} \mu^{-}$for different degrees of polarization. The study was made at $\sqrt{s}=650 \mathrm{GeV}$ for $m_{\tilde{\nu}}=650 \mathrm{GeV}, \Gamma_{\tilde{\nu}}=1 \mathrm{GeV}$, an angular cut of $45^{\circ} \leq \theta \leq 135^{\circ}$ and the R-parity-violating couplings $\lambda_{131}=0.05$ and $\lambda_{232}=0.05$, respectively [134].

of both beams with $\left(P_{e^{-}}, P_{e^{+}}\right)=(-80 \%,-60 \%)$ produces an increase of about $20-$ $30 \%$.

\subsubsection{Production of heavy Higgs bosons in the MSSM}

Searches for heavy SUSY Higgs particles can be extremely challenging for both the LHC and the ILC. Exploiting single Higgs-boson production in $e^{+} e^{-} \rightarrow \nu \bar{\nu} H$ extends the kinematical reach considerably. However, in the decoupling region, $m_{A} \gg m_{Z}$, the suppressed couplings of the heavy Higgs boson to SM gauge bosons lead to very small rates. This difficulty could be attenuated by accumulating a very high integrated luminosity, together with a further enhancement of the signal cross section by polarizing both beams.

The possibility to enhance cross sections with beam polarization can be very important 
for detecting processes with a low rate. An example has been worked out in [137], where the production of the heavy neutral CP-even Higgs boson $H$ of the MSSM was studied. The MSSM Higgs sector is characterized by two parameters at tree-level, the mass of the pseudoscalar Higgs boson, $m_{A}$, and $\tan \beta$. The CP-even Higgs bosons $h, H$ share their couplings to the gauge bosons, while the couplings of the $\mathrm{CP}$-odd Higgs boson $A$ to two gauge bosons vanishes.

Over large parts of the whole parameter space, i.e. $m_{A} \gg m_{Z}$, the lightest CP-even Higgs boson, $h$, of the MSSM is SM-like, and the coupling of the heavy CP-even Higgs boson to two gauge bosons is suppressed [75]. In this case only the pair production channel $e^{+} e^{-} \rightarrow H A$ contributes at full strength. Since for large values of $m_{A}$ the heavy Higgs bosons $A$ and $H$ are approximately mass degenerate, $m_{A} \approx m_{H}$, the pair production channel $e^{+} e^{-} \rightarrow H A$ is limited by kinematics to the region $m_{H}<\sqrt{s} / 2$. If the rare process $e^{+} e^{-} \rightarrow \nu \bar{\nu} H$, governed by the suppressed coupling of $H$ to two gauge bosons, can be exploited, the kinematic reach of the linear collider can be extended.

In [137] it was shown that higher-order contributions to the couplings of the heavy Higgs boson to the gauge bosons can remedy the suppression. This leads to considerably higher cross sections in certain domains of the MSSM parameter space and makes the process potentially accessible at the linear collider. This requires a high integrated luminosity and polarized beams. The cross section is enhanced for left-handed electrons and right-handed positrons. While an $80 \%$ polarization of the electron beam results in a cross section that is enhanced by a factor 1.8 , the polarization of both beams, i.e. $80 \%$ polarization for electrons and $60 \%$ for positrons, would roughly yield an enhancement by a factor of 2.9. Therefore, with polarization of both beams, this process may be measurable. With the ILC running at the high energy of $1 \mathrm{TeV}$, the enhancement of the cross section by the beam polarization can extend the kinematic reach by roughly $100 \mathrm{GeV}$, see fig. 3.21 (right), with respect to the case of unpolarized beams, fig. 3.21 (left). Here the SUSY parameters are chosen as in scenario S15, cf. table 3.1. It has been assumed that at least about 20 heavy Higgs boson events have to be observed in order to establish this channel. This corresponds to an integrated luminosity of the order of $2 \mathrm{ab}^{-1}$ for a production cross section larger than $0.1 \mathrm{fb}$ (left dashed and dark regions in fig. 3.21).

Quantitative example: Using $\left(P_{e^{-}}, P_{e^{+}}\right)=(-80 \%,+60 \%)$ instead of $(-80 \%, 0)$, one gains about a factor $\mathbf{1 . 6}$ for the signal cross sections. Therefore the polarization of both beams could be decisive to accumulate enough statistics to observe signals of the heavy SUSY Higgs bosons.

\subsection{Effective contact-interactions and heavy gauge bosons}

Effective contact-interactions (CI) represent a general tool for parametrizing at 'low-energy' the effects of non-standard dynamics characterized by exchanges, among the SM particles, of very high-mass states, much higher than the available accelerator energy. Manifestations of such new interactions can therefore be searched for only through deviations of cross sections from the SM predictions, and 'indirect' bounds on the new energy-scales and coupling constants can be derived. Denoting by $\Lambda$ the above-mentioned new large scales, for dimensional reasons the deviations of the observables from the SM predictions can be suppressed by powers of the ratio $\sqrt{s} / \Lambda$ which should be smaller that unity for the effective theory to be a reliable description. Such effects are therefore expected to be 

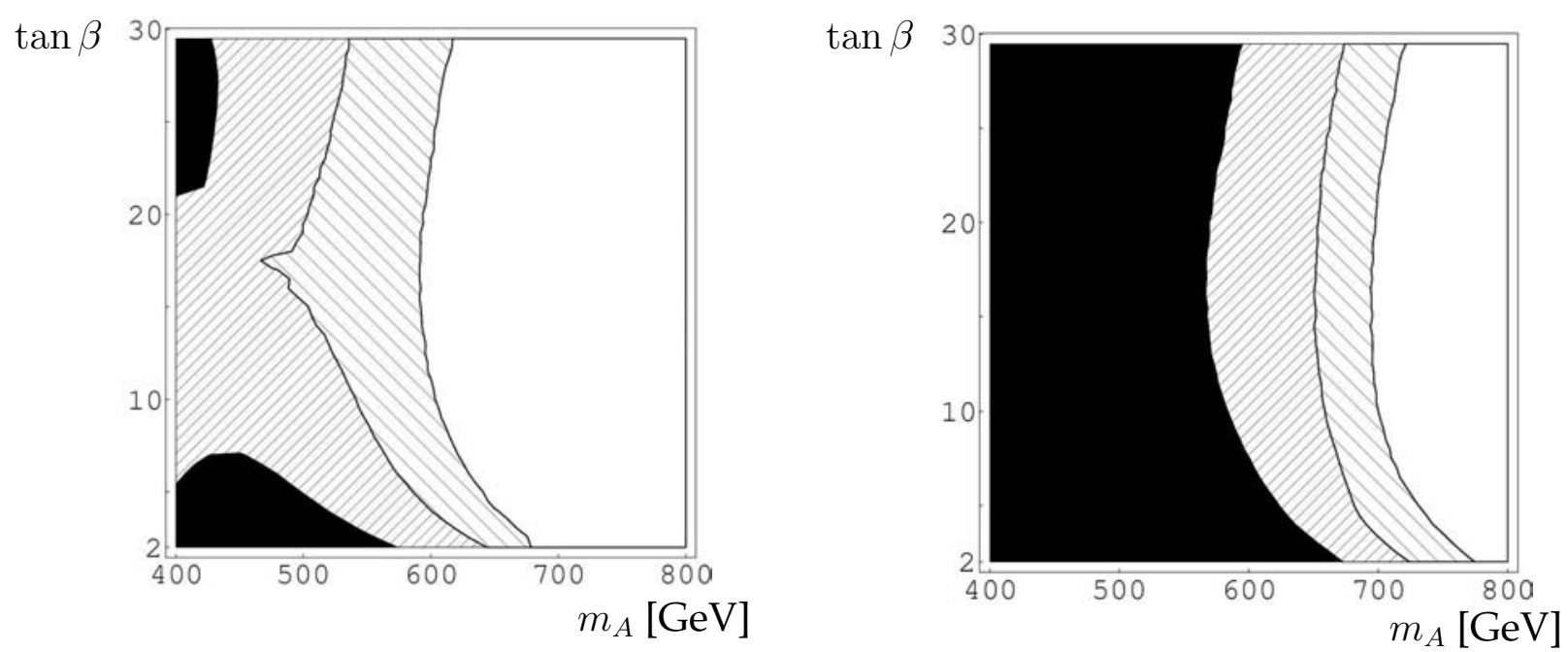

Figure 3.21: Cross section of heavy Higgs production $\sigma\left(e^{+} e^{-} \rightarrow \nu \bar{\nu} H\right)$ for the scenario S15, table 3.1, in the $m_{A}-\tan \beta$ plane with $\sigma>0.05 \mathrm{fb}$ (black), $>0.02 \mathrm{fb}$ (right dashed) and $>0.01 \mathrm{fb}$ (left dashed) for unpolarized beams (left) and with both beams polarized, $\left(P_{e^{-}}, P_{e^{+}}\right)=(-80 \%,+60 \%)$ (right) at $\sqrt{s}=1 \mathrm{TeV}$ [137].

small, and favoured at high-energy and high-luminosity machines.

\subsubsection{Analysis of four-fermion contact-interactions}

Longitudinal beam polarization of both beams is decisive to derive model-independent bounds on the different possible couplings. With both beams polarized, the error in $P_{\text {eff }}$ is reduced and the accuracy of the $A_{\mathrm{LR}}$ measurement is considerably enhanced and more observables can be defined. The systematic errors can be significantly reduced with both beams polarized, which is of crucial value.

In the following it is focused on the production of Standard Model fermion-pairs. Although such processes are not primarily devoted at the LC to the search of new phenomena, they guarantee a good sensitivity to exchanges of heavy mass scales $\Lambda \gg m_{W, Z}$ thanks to the clear signature of the final states in the detector and to the available high statistics.

For the fermion-pair production process

$$
e^{+}+e^{-} \rightarrow f+\bar{f}
$$

the general, $S U(3) \times S U(2) \times U(1)$ symmetric eef $f$ CI Lagrangian (dimension $D=6$ ) with helicity-conserving and flavour-diagonal fermion currents, was proposed in [138]:

$$
\mathcal{L}_{\mathrm{CI}}=\frac{1}{1+\delta_{e f}} \sum_{i, j} g_{\mathrm{eff}}^{2} \epsilon_{i j}\left(\bar{e}_{i} \gamma_{\mu} e_{i}\right)\left(\bar{f}_{j} \gamma^{\mu} f_{j}\right)
$$

In eq. (3.25), $i, j=\mathrm{L}, \mathrm{R}$ denote left- or right-handed helicities (see below), generation and colour indices have been suppressed, and the CI coupling constants are parametrized in terms of corresponding mass scales as $\epsilon_{i j}=\eta_{i j} / \Lambda_{i j}^{2}$ with $\eta_{i j}= \pm 1,0$, depending on the chiral structure of the individual interactions. Also, conventionally $g_{\text {eff }}^{2}=4 \pi$ is assumed, as a reminder that, in the case of compositeness, the new interaction would become strong at 
$\sqrt{s}$ of the order of $\Lambda_{i j}$. Obviously, deviations from the SM and upper bounds or exclusion ranges for the $\mathrm{CI}$ couplings can be equivalently expressed as lower bounds and exclusion ranges for the corresponding mass scales $\Lambda_{i j}$.

A general, model-independent analysis of the process (3.24) in terms of the interaction (3.25) must simultaneously account for all CI couplings as free, non-vanishing parameters. On the other hand, one would like to set, from data, separate constraints on the $\Lambda$ s.

A simplifying procedure is to assume non-zero values for only one of the couplings (or one specific combination of them) at a time when all others are set to zero, to test specific CI models only.

With longitudinally-polarized beams, the analysis of contact interactions can make use of observables integrated over the polar scattering angle: the (unpolarized) total cross section $\sigma_{0}$ and forward-backward asymmetry $A_{\mathrm{FB}}$, the left-right asymmetry $A_{\mathrm{LR}}$ and left-right forward-backward asymmetry $A_{\mathrm{LR}, \mathrm{FB}}$. These are defined in this subsection in the notation of [139], which differs from the notation in eqs. (1.15)-(1.18), (1.22), (1.23) in section 1.2.1: the first and second subscripts refer to the helicities of the incoming and outgoing fermion respectively. Therefore the components are denoted with $\hat{\sigma}$ :

$$
\begin{gathered}
\sigma_{0}=\frac{1}{4}\left[\hat{\sigma}_{\mathrm{LL}}+\hat{\sigma}_{\mathrm{LR}}+\hat{\sigma}_{\mathrm{RR}}+\hat{\sigma}_{\mathrm{RL}}\right], \\
A_{\mathrm{FB}}=\frac{3}{4} \frac{\hat{\sigma}_{\mathrm{LL}}-\hat{\sigma}_{\mathrm{LR}}+\hat{\sigma}_{\mathrm{RR}}-\hat{\sigma}_{\mathrm{RL}}}{\hat{\sigma}_{\mathrm{LL}}+\hat{\sigma}_{\mathrm{LR}}+\hat{\sigma}_{\mathrm{RR}}+\hat{\sigma}_{\mathrm{RL}}}, \\
A_{\mathrm{LR}}=\frac{\hat{\sigma}_{\mathrm{LL}}+\hat{\sigma}_{\mathrm{LR}}-\hat{\sigma}_{\mathrm{RR}}-\hat{\sigma}_{\mathrm{RL}}}{\hat{\sigma}_{\mathrm{LL}}+\hat{\sigma}_{\mathrm{LR}}+\hat{\sigma}_{\mathrm{RR}}+\hat{\sigma}_{\mathrm{RL}}}, \\
A_{\mathrm{LR}, \mathrm{FB}}=\frac{3}{4} \frac{\hat{\sigma}_{\mathrm{LL}}-\hat{\sigma}_{\mathrm{RR}}+\hat{\sigma}_{\mathrm{RL}}-\hat{\sigma}_{\mathrm{LR}}}{\hat{\sigma}_{\mathrm{LL}}+\hat{\sigma}_{\mathrm{RR}}+\hat{\sigma}_{\mathrm{RL}}+\hat{\sigma}_{\mathrm{LR}}} .
\end{gathered}
$$

The deviations of measurements of these observables from the SM predictions are expressed in terms of the CI, $\epsilon_{i j}$, of eq. (3.25).

It can be seen from eqs. (3.26) and (3.27) that, with unpolarized beams, the CI couplings could not be individually constrained within finite ranges, but only mutual correlations could be derived. With longitudinal beam polarization, the two additional physical observables, (3.28) and (3.29), are available to obtain finite, model-independent bounds on all CI couplings [140-142].

In principle, the polarization of the electron beam alone would be sufficient to achieve model-independent results. The polarization of both beams increases the cross sections (see section 1.3) and improves the sensitivity to the new parameters which in general scales for dimension $\mathrm{D}=6$ operators such as (3.25), with

$$
\frac{m_{X}}{g_{X}} \sim \sqrt{\Delta_{\text {stat }} \sigma} \sim\left(\mathcal{L}_{\text {int }} \cdot s\right)^{1 / 4}
$$

taking into account statistical errors only. Further, with both beams polarized, the error of the effective polarization, $P_{\text {eff }}$ (see also section 1.2.3), is substantially reduced and involves a higher accuracy of the $A_{\mathrm{LR}}$ measurement:

$$
\Delta A_{\mathrm{LR}}=\sqrt{\left(\Delta_{\mathrm{stat}} A_{\mathrm{LR}}\right)^{2}+\left(\Delta_{\mathrm{sys}} A_{\mathrm{LR}}\right)^{2}}=\sqrt{\frac{1-P_{\mathrm{eff}}^{2} A_{\mathrm{LR}}^{2}}{N P_{\mathrm{eff}}^{2}}+A_{\mathrm{LR}}^{2}\left(\frac{\Delta P_{\mathrm{eff}}}{P_{\mathrm{eff}}}\right)^{2}} .
$$




\section{Expected sensitivities to contact interactions}

The analysis of CI with $f \neq e, t$ in ref. [140] demonstrates the sensitivity to $\Lambda$ depending on the linear collider parameters. To assess the relative roles of statistical and systematic uncertainties, the time-integrated luminosity $\mathcal{L}_{\text {int }}$ is varied from 50 to $500 \mathrm{fb}^{-1}$, with uncertainty $\Delta \mathcal{L}_{\text {int }} / \mathcal{L}_{\text {int }}=0.5 \%$, and a cut $|\cos \theta| \leq 0.99$ is assumed. The polarization of electron and positron beams is considered as $\left(\left|P_{e^{-}}\right|,\left|P_{e^{+}}\right|\right)=(80 \%, 0)$ and $\left(\left|P_{e^{-}}\right|,\left|P_{e^{+}}\right|\right)=$ $(80 \%, 60 \%)$, with the uncertainties $\Delta P_{e^{-}} / P_{e^{-}}=\Delta P_{e^{+}} / P_{e^{+}}=0.5 \%$.

The model-independent bounds on the mass scales $\Lambda_{i j}$ in the final $b \bar{b}$ and $c \bar{c}$ cases, allowed by these experimental uncertainties, are shown in fig. 3.22. Particle identification efficiencies of $60 \%$ and $30 \%$ are assumed in the $b \bar{b}$ and $c \bar{c}$ channels, respectively [143]. Thick lines in fig. 3.22 correspond to $\left(\left|P_{e^{-}}\right|,\left|P_{e^{+}}\right|\right)=(80 \%, 60 \%)$ while thin lines correspond to $\left(\left|P_{e^{-}}\right|,\left|P_{e^{+}}\right|\right)=(80 \%, 0)$.
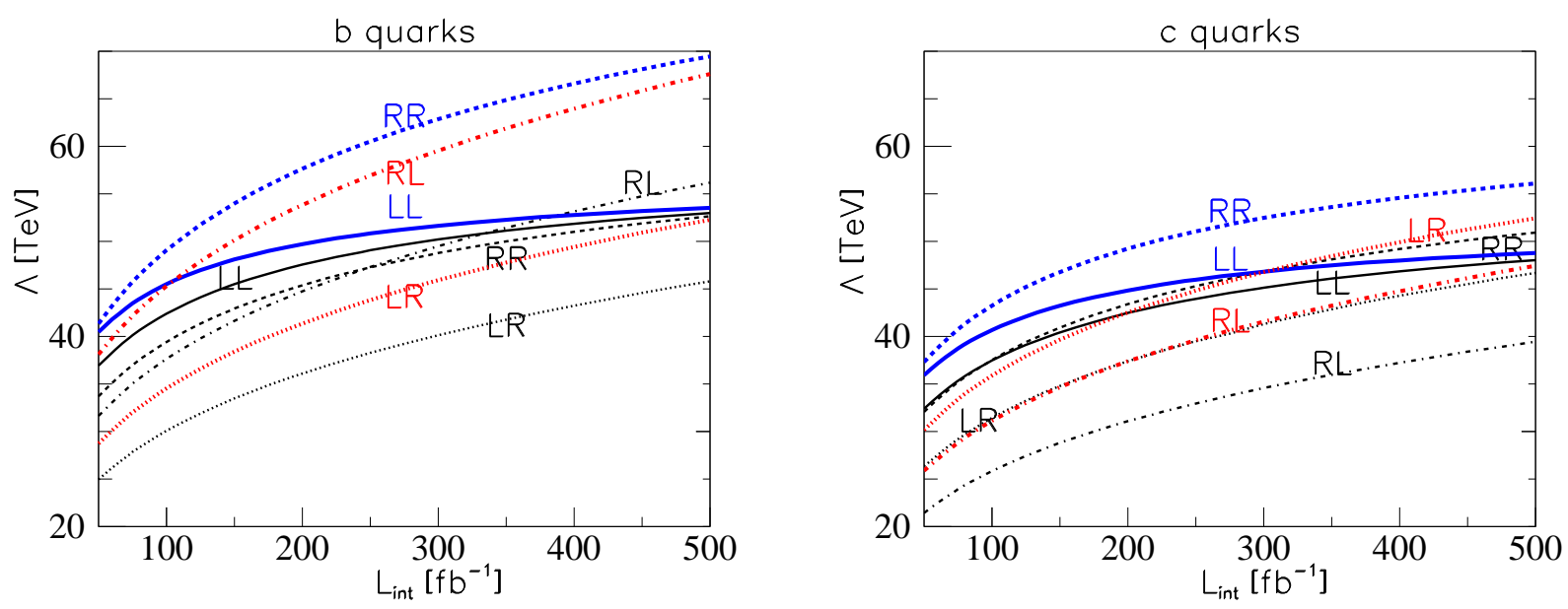

Figure 3.22: Lower bounds (95\% C.L.) on the scale of CI, $\Lambda$, at $\sqrt{s}=500 \mathrm{GeV}$ vs. the integrated luminosity, $\mathcal{L}_{\text {int }}$, for $b \bar{b}$ and $c \bar{c}$ final states taking into account the four helicity combinations. Thin lines: $\left(\left|P_{e^{-}}\right|,\left|P_{e^{+}}\right|\right)=(80 \%, 0)$, thick lines: $\left(\left|P_{e^{-}}\right|,\left|P_{e^{+}}\right|\right)=$ $(80 \%, 60 \%)$ [140].

In fig. 3.23 the expected sensitivities for different models of contact interactions in $e^{+} e^{-} \rightarrow b \bar{b}, c \bar{c}$ are shown, including systematic $\left(\Delta_{\text {syst }}=0 \%, 0.5 \%, 1.0 \%\right)$, luminosity $\left(\Delta \mathcal{L}_{\text {int }}=0.2 \%, 0.5 \%\right)$ and polarization uncertainties $\left(\Delta P_{e^{-}} / P_{e^{-}}=\Delta P_{e^{+}} / P_{e^{+}}=0 \%, 0.5 \%\right)$. It can clearly be seen that the reduction of systematic errors will be decisive to extend the reach on the $\Lambda \mathrm{s}$. This study was done for $\sqrt{s}=800 \mathrm{GeV}$ [144].

\section{Contact-interaction analysis in Bhabha scattering}

With $\delta_{e f}=1$ the four-fermion contact-interaction Lagrangian of eq. (3.25) is relevant to the Bhabha scattering process $e^{+} e^{-} \rightarrow e^{+} e^{-}$, where $\gamma$ and $Z$ bosons are exchanged in both $s$ - and $t$-channels. It turns out that modifications of the pure $t$-channel contribution to the cross section, $\hat{\sigma}_{\mathrm{LR}, t}$, depend on the single CI parameter $\left(\epsilon_{\mathrm{LR}}=\epsilon_{\mathrm{RL}}\right)$, while the combinations of helicity cross sections, $\mathrm{d} \hat{\sigma}_{\mathrm{R}}$ and $\mathrm{d} \hat{\sigma}_{\mathrm{L}}$, contribute to the $s$-channel exchange and depend on pairs of parameters, $\left(\epsilon_{\mathrm{RR}}, \epsilon_{\mathrm{LR}}\right)$ and $\left(\epsilon_{\mathrm{LL}}, \epsilon_{\mathrm{LR}}\right)$, respectively. 

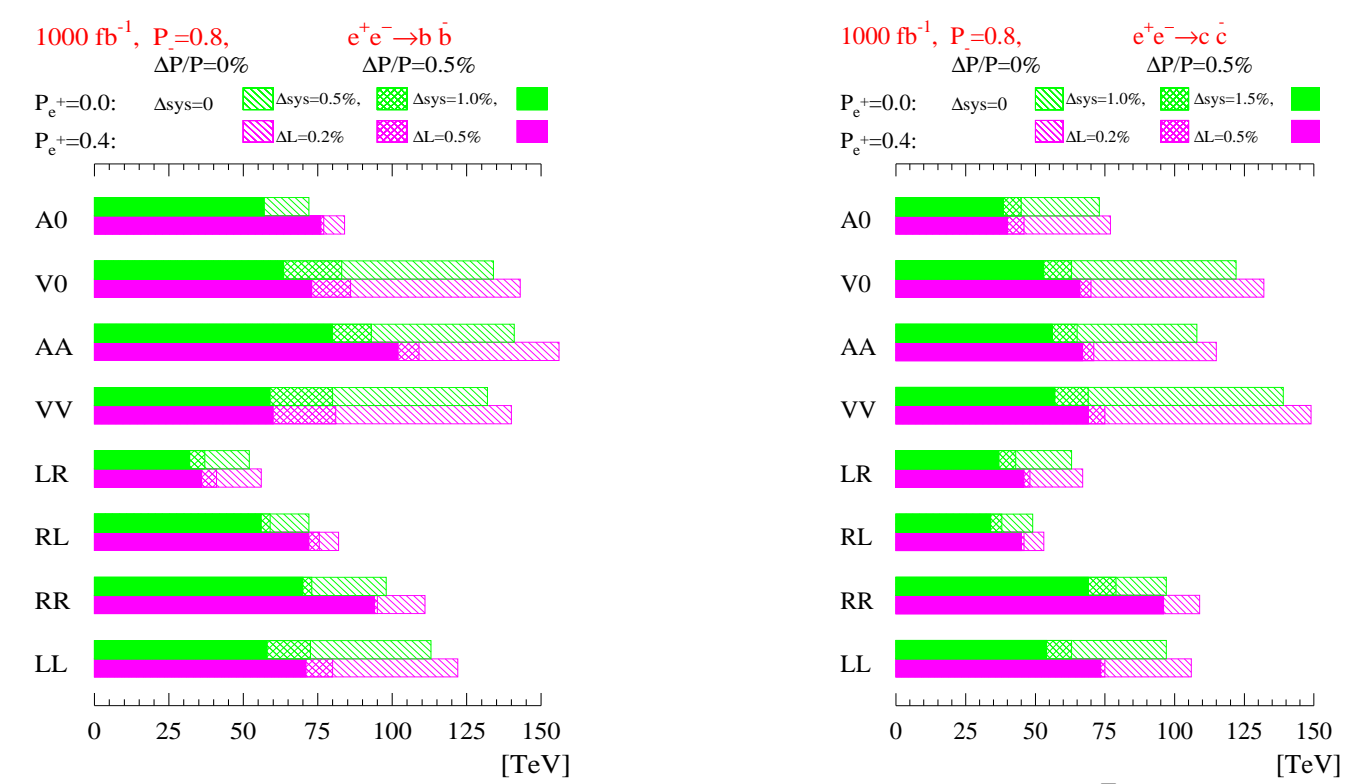

Figure 3.23: Limits on contact interactions from $e^{+} e^{-} \rightarrow b \bar{b}$ and $e^{+} e^{-} \rightarrow c \bar{c}$ without positron polarization (green) and with $40 \%$ polarization (margenta) for the different uncertainty scenarios $\Delta P_{e^{ \pm}} / P_{e^{ \pm}}=0, \Delta$ sys $=0, \Delta L_{\text {int }}=0$ (single hatched), $\Delta P_{e^{ \pm}} / P_{e^{ \pm}}=$ $0.5 \%, \Delta$ sys $=0.5 \%, \Delta L=0.2 \%$ (double hatched) and $\Delta P_{e^{ \pm}} / P_{e^{ \pm}}=0.5 \%, \Delta$ sys $=1.0 \%$, $\Delta L_{\text {int }}=0.5 \%$ (filled) at $\sqrt{s}=500 \mathrm{GeV}$ and $\mathcal{L}_{\text {int }}=1 \mathrm{ab}^{-1}$ [144].

The change of the polarization of each beam allows the separate measurements of the polarized differential cross sections $\mathrm{d} \sigma_{++}, \mathrm{d} \sigma_{+-}$and $\mathrm{d} \sigma_{-+}$, cf. discussion following eq. (1.15) [145]. These differential cross sections represent a system of linear equations of the helicity cross sections $\mathrm{d} \hat{\sigma}_{\mathrm{R}}, \mathrm{d} \hat{\sigma}_{\mathrm{L}}$ and $\hat{\sigma}_{\mathrm{LR}, t}$ and allow the CI couplings $\epsilon_{\mathrm{LL}}, \epsilon_{\mathrm{RR}}$ and $\epsilon_{\mathrm{LR}}$ to be disentangled. Equations (3.26)-(3.29) show that this kind of model-independent analysis requires both $e^{+}$and $e^{-}$polarized. Fig. 3.24 shows as an example the result of a $\chi^{2}$ analysis assuming that no deviation from the SM within the experimental uncertainty (statistical and systematic) is measured in $\mathrm{d} \hat{\sigma}_{\mathrm{L}}, \mathrm{d} \hat{\sigma}_{\mathrm{R}}$ and $\mathrm{d} \hat{\sigma}_{\mathrm{LR}, t}$ for $\mathcal{L}_{\text {int }}\left(e^{+} e^{-}\right)=50 \mathrm{fb}^{-1}$, $\left(\left|P_{e^{-}}\right|,\left|P_{e^{+}}\right|\right)=(80 \%, 60 \%), \Delta \mathcal{L}_{\text {int }} / \mathcal{L}_{\text {int }}=\Delta P_{e^{-}} / P_{e^{-}}=\Delta P_{e^{+}} / P_{e^{+}}=0.5 \%$.

A comparison with Møller scattering [145] shows that only in the case where $\mathcal{L}_{\text {int }}\left(e^{-} e^{-}\right)$ is not too low are Bhabha and Møller scattering complementary as regards the sensitivity to individual couplings in a model-independent data analysis.

\section{Sensitivity to neutral extra gauge bosons}

Extra neutral gauge bosons $Z^{\prime}$ can be probed by their virtual effects on cross sections and asymmetries by replacing $\epsilon_{i j} \rightarrow g_{i}^{\prime} g_{j}^{\prime} /\left(s-M_{Z^{\prime}}^{2}\right)$. For energies below a $Z^{\prime}$ resonance, measurements of fermion-pair production are sensitive only to this ratio of $Z^{\prime}$ couplings and $Z^{\prime}$ mass. Therefore, limits on the $Z^{\prime}$ mass can be obtained only in dependence on a model with given $Z^{\prime}$ couplings (for reviews see, e.g., $[146,147]$ ). For example, for the well-known $\mathrm{E}_{6}$ and LR models, mass sensitivities between $4 \times \sqrt{s}$ and $14 \times \sqrt{s}$ are reached. Thus, the LC operating at $\sqrt{s}=800 \mathrm{GeV}$ may exceed the sensitivity of the LHC (which is about $4-5 \mathrm{TeV}$ ) to a potential $Z^{\prime}$ in some models. Conversely, if a $Z^{\prime}$ will be detected at the LHC its origin can be found by determining the $Z^{\prime}$ couplings, see fig. 3.25 (left) [148]. While positron-beam polarization improves only slightly the resolution power for $Z^{\prime}$ models 

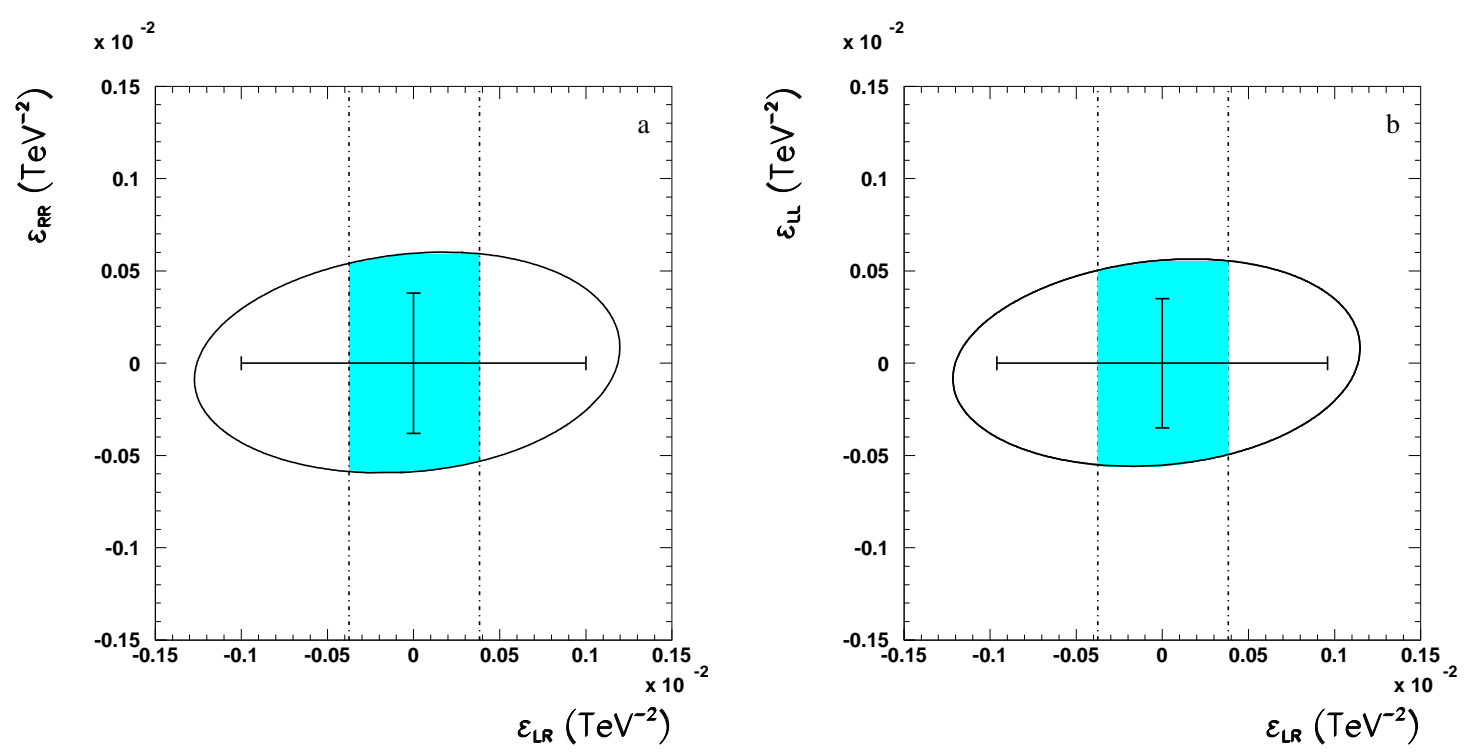

Figure 3.24: Allowed areas at 95\% C.L. in the planes $\left(\epsilon_{\mathrm{LR}}, \epsilon_{\mathrm{RR}}\right)$ and $\left(\epsilon_{\mathrm{LR}}, \epsilon_{\mathrm{LL}}\right)$ obtained from $\hat{\sigma}_{\mathrm{R}}$ and $\hat{\sigma}_{\mathrm{L}}$ in $e^{+} e^{-} \rightarrow e^{+} e^{-}$at $\sqrt{s}=0.5 \mathrm{TeV}, \mathcal{L}_{\text {int }}\left(e^{+} e^{-}\right)=50 \mathrm{fb}^{-1},\left(\left|P_{e^{-}}\right|,\left|P_{e^{+}}\right|\right)=$ $(80 \%, 60 \%)$. Vertical dashed lines indicate the range allowed for $\epsilon_{\mathrm{LR}}$ by $\hat{\sigma}_{\mathrm{LR}, t}$. The crosses indicate the constraints obtained by taking only one non-zero parameter at a time instead of two simultaneously non-zero and independent parameters [145].

in the case of leptonic final states, it will be important for the measurement of the $Z^{\prime}$ couplings to quarks. The polarization of both beams is also important to enhance the resolution power considering hadronic final states. In fig. 3.25 (right) the reconstruction of the $Z^{\prime}$ model is demonstrated without knowledge of the $Z^{\prime}$ mass based on $b \bar{b}$ final states. In these analyses the crucial point is the fact that the systematic errors can be significantly reduced when both beams are polarized [144,148].

Quantitative examples: With the polarization of both beams the sensitivity to the new physics scale can be increased by a factor of up to about 1.3 with respect to the case with only polarized electrons. A similar gain is reached for the resolution power for $Z^{\prime}$ studies in different models. The determination of $Z^{\prime}$ couplings can be substantially improved by up to about a factor 1.5. The crucial systematic errors can be significantly reduced when both beams are polarized.

\subsubsection{Transversely-polarized beams and leptoquark searches}

With transversely-polarized beams, CP-conserving and CP-violating azimuthal asymmetries of the final-state top quark in $e^{+} e^{-} \rightarrow t \bar{t}$ can be used to probe a scalar leptoquark model.

It was pointed out in refs. $[9,149]$ that transverse polarization can play a unique role in isolating chirality-violating couplings, such as scalar or tensorial ones, to which longitudinally-polarized beams are not sensitive. The interference of new chirality-violating contributions with the chirality-conserving standard model (SM) couplings give rise to terms in the angular distribution proportional to $\sin \theta \cos \phi$ and $\sin \theta \sin \phi$, where $\theta$ and $\phi$ are the polar and azimuthal angles of a final-state particle. Chirality-conserving new cou- 

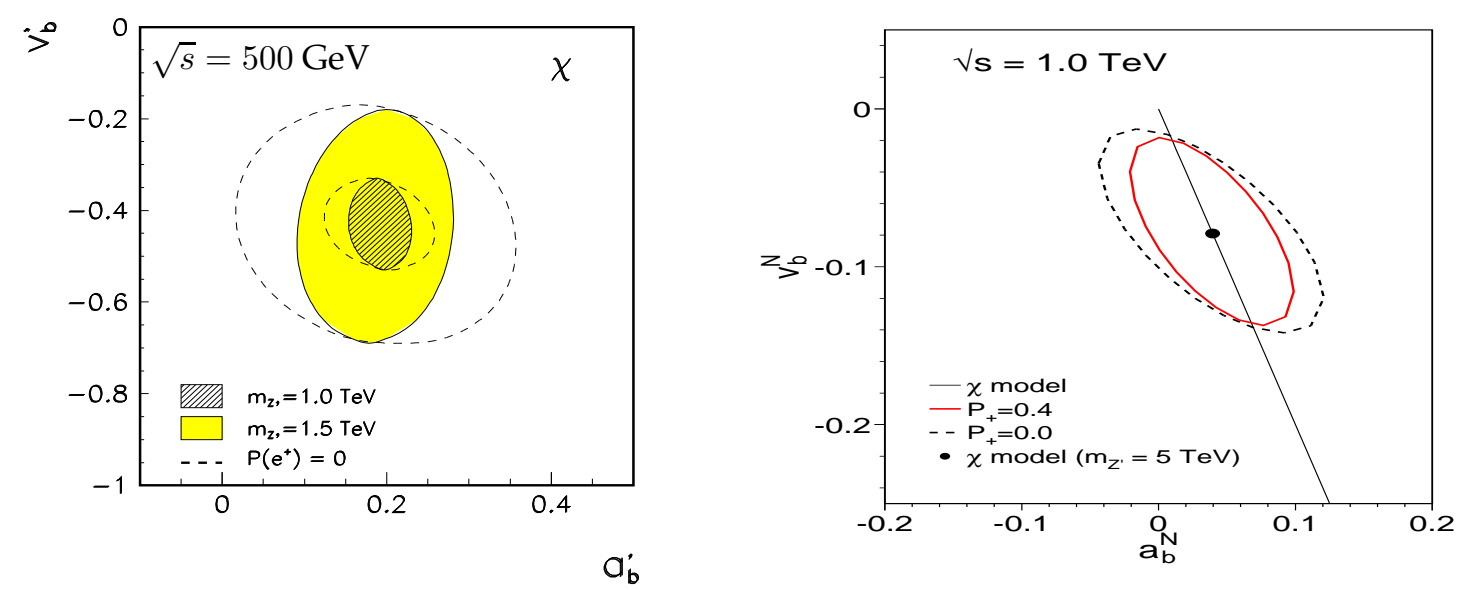

Figure 3.25: Left: 95\% C.L. contours for the axial $\left(a_{b}^{\prime}\right)$ and vector $\left(v_{b}^{\prime}\right)$ couplings of the $Z^{\prime}$ for $M_{Z^{\prime}}=1.0,1.5 \mathrm{TeV}$ in the $\chi$ model with $\sqrt{s}=500 \mathrm{GeV}$ and $\mathcal{L}_{\text {int }}=500 \mathrm{fb}^{-1}$. The dashed lines correspond to $P_{e^{+}}=0$ [148]; $a_{b}^{\prime}, v_{b}^{\prime}$ denote the couplings of $Z^{\prime}$ to the $b$-quarks. Right: Expected resolution power (95\% C.L.) to reconstruct $b \bar{b}$ couplings of $Z^{\prime}\left(m_{Z^{\prime}}=\right.$ $5 \mathrm{TeV})$ realized in the $\chi$ model based on the measurement of $b \bar{b}$ final states. Here, the configuration $\left(P_{e^{-}}, P_{e^{+}}\right)=(80 \%, 40 \%)$ is compared with $\left(P_{e^{-}}, P_{e^{+}}\right)=(80 \%, 0)$. The $Z^{\prime}$ mass is assumed to be unknown in this case [144]. The normalized couplings are defined as $a_{b}^{\mathrm{N}}=a_{b}^{\prime} \sqrt{s /\left(m_{Z^{\prime}}^{2}-s\right)}$ and $v_{b}^{\mathrm{N}}=v_{b}^{\prime} \sqrt{s /\left(m_{Z^{\prime}}^{2}-s\right)}$.

plings, on the other hand, produce interference contributions proportional to $\sin ^{2} \theta \cos 2 \phi$ and $\sin ^{2} \theta \sin 2 \phi$. Chirality-violating contributions do not interfere with the chiralityconserving SM contribution with unpolarized or longitudinally-polarized beams when the electron mass is neglected. Hence transverse polarization would enable the measurement of chirality-violating couplings through the azimuthal distributions and the unambiguous distinction from chirality-conserving new interactions.

An example [150] is represented by a specific model where chirality violation appears already at the tree level, by extending the SM gauge group by a $S U(2)_{L}$ doublet of scalar leptoquarks (LQ), which couples only to first-generation leptons and third-generation quarks. Since the couplings of leptoquarks to the third generation quarks are relatively weakly constrained [151], their effect from $t$-channel exchange in $e^{+} e^{-} \rightarrow t \bar{t}$ can be nonnegligible. The leptoquark couplings of both left and right chiralities are included in the model, $g_{L}$ and $g_{R}$, and they are also allowed to be complex. Thus, in principle, also the possibility of $\mathrm{CP}$ violation can be kept open. The reader is referred to [152] for a general discussion of leptoquark models, and to [153] for a brief review of quantum numbers.

Assume transverse polarizations $P_{e^{-}}^{\mathrm{T}}$ and $P_{e^{+}}^{\mathrm{T}}$ that are (anti)parallel to each other. The differential cross section for the process $e^{+} e^{-} \rightarrow t \bar{t}$ is here given by the sum of the SM contribution $\sigma_{\mathrm{SM}}$, the pure leptoquark contribution $\sigma_{\mathrm{LQ}}$, and the contribution $\sigma_{\text {int }}$ from the interference of the leptoquark contribution with the SM contribution. The interference term between the SM $Z$ and the leptoquark contribution contains terms linear in $P_{e^{-}}^{\mathrm{T}}$ and $P_{e^{+}}^{\mathrm{T}}$ that are proportional to $\sin \theta \cos \phi$ and $\sin \theta \sin \phi$. These terms are proportional to the real and imaginary parts of the chirality-violating couplings $g_{R} g_{L}^{*}$, respectively, cf. the analytical expressions in [150], thus, in principle, also their relative phases can be measured.

The interference terms that are bilinear in $P_{e^{-}}^{\mathrm{T}}$ and $P_{e^{+}}^{\mathrm{T}}$ (thus requiring both beams to 
be polarized) and proportional to $\sin ^{2} \theta \sin 2 \phi$ and $\sin ^{2} \theta \cos 2 \phi$ contain $\left|g_{L}\right|^{2}$ or $\left|g_{R}\right|^{2}$. These terms, however, can be studied also using longitudinal polarization.

The interference terms proportional to $\sin \theta \cos \phi$ and $\sin \theta \sin \phi$ are linear in $P_{e^{-}}^{\mathrm{T}}$ and $P_{e^{+}}^{\mathrm{T}}$ (thus not strictly requiring both beams polarized), and are proportional to $\operatorname{Re}\left(g_{R} g_{L}^{*}\right)$ and the CP-violating $\operatorname{Im}\left(g_{R} g_{L}^{*}\right)$, respectively.

The chirality-violating terms can be isolated by studying the following azimuthal asymmetries, where $\theta$ is integrated over with a cut-off $\theta_{0}$ in the forward and backward directions:

$$
\begin{gathered}
A_{1}\left(\theta_{0}\right)=\frac{1}{r \sigma\left(\theta_{0}\right)} \int_{-\cos \theta_{0}}^{\cos \theta_{0}} d \cos \theta\left[\int_{0}^{\pi} d \phi-\int_{\pi}^{2 \pi} d \phi\right] \frac{d \sigma}{d \Omega} \\
A_{2}\left(\theta_{0}\right)=\frac{1}{\sigma\left(\theta_{0}\right)} \int_{-\cos \theta_{0}}^{\cos \theta_{0}} d \cos \theta\left[\int_{0}^{\pi / 2} d \phi-\int_{\pi / 2}^{3 \pi / 2} d \phi+\int_{3 \pi / 2}^{2 \pi} d \phi\right] \frac{d \sigma}{d \Omega}
\end{gathered}
$$

where

$$
\sigma\left(\theta_{0}\right)=\int_{-\cos \theta_{0}}^{\cos \theta_{0}} d \cos \theta \int_{0}^{2 \pi} d \phi \frac{d \sigma}{d \Omega}
$$

It turns out that $A_{1}\left(\theta_{0}\right)$ and $A_{2}\left(\theta_{0}\right)$ differ only in the factors $\left(P_{e^{-}}^{\mathrm{T}}-P_{e^{+}}^{\mathrm{T}}\right) \operatorname{Im}\left(g_{R} g_{L}^{*}\right)$ and $\left(P_{e^{-}}^{\mathrm{T}}+P_{e^{+}}^{\mathrm{T}}\right) \operatorname{Re}\left(g_{R} g_{L}^{*}\right)$.

A numerical study has been done for $\sqrt{s}=500 \mathrm{GeV}$ and $\mathcal{L}_{\text {int }}=500 \mathrm{fb}^{-1}$. Furthermore, $\left(P_{e^{-}}^{\mathrm{T}}, P_{e^{+}}^{\mathrm{T}}\right)=(80 \%,-60 \%), g_{L}=1 / \sqrt{2}, g_{R}=i / \sqrt{2}$ which corresponds to maximal CP violation in the leptoquark couplings and a leptoquark mass $M_{\mathrm{LQ}}=1 \mathrm{TeV}$. The asymmetry $A_{1}\left(\theta_{0}\right)$ is in this case of the order of $4 \times 10^{-3}$, and is not very sensitive to the cut-off $\theta_{0}$. In the case without CP violation, $g_{L}=1 / \sqrt{2}$ and $g_{R}=1 / \sqrt{2}$, the sign of $P_{e^{+}}^{\mathrm{T}}$ is chosen positive to maximize the asymmetry and one obtains the same size for $A_{2}\left(\theta_{0}\right)$.
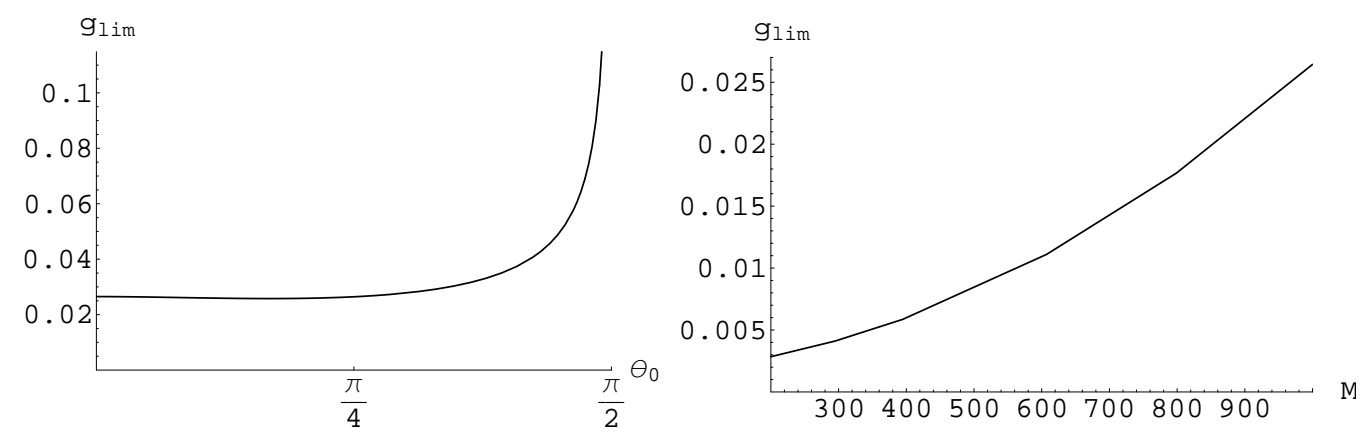

Figure 3.26: The 90\% CL limit $g_{\text {lim }}$ that can be obtained on $\operatorname{Re}\left(g_{R} g_{L}^{*}\right)$ or $\operatorname{Im}\left(g_{R} g_{L}^{*}\right)$ respectively from $A_{1}$ or $A_{2}$ for $\sqrt{s}=500 \mathrm{GeV}$ and $\mathcal{L}_{\text {int }}=500 \mathrm{fb}^{-1}$ vs. $\theta_{0}$ (left panel) and $M[\mathrm{GeV}]$ (right panel) [150].

Fig. 3.26 shows the $90 \%$ C.L. limit $g_{\text {lim }}$ that can be put on the combinations $\operatorname{Im}\left(g_{R} g_{L}^{*}\right)$ (in the maximal CP violation case) and $\operatorname{Re}\left(g_{R} g_{L}^{*}\right)$ (in the CP conservation case). This limit is obtained by equating the asymmetry to $1.64 / \sqrt{N_{\mathrm{SM}}}$, where $N_{\mathrm{SM}}$ is the number of SM events, $N_{\mathrm{SM}}=\sigma_{\mathrm{SM}}\left(\theta_{0}\right) \mathcal{L}_{\text {int }}$. The possible limit $g_{\text {lim }}$ on $\operatorname{Re}\left(g_{R} g_{L}^{*}\right)$ or $\operatorname{Im}\left(g_{R} g_{L}^{*}\right)$ is about $2.5 \times$ $10^{-2}$ for most values of $\theta_{0} \leq \pi / 4$ and $M_{\mathrm{LQ}}=1000 \mathrm{GeV}$.

These are 'direct' limits. In the case where the leptoquark has both left- and righthanded couplings, strong indirect limits exist [154,155], the most stringent ones coming 
from dipole moments of the electron. Requiring the contribution to the electron $g_{e}-2$ coming from one-loop diagrams with top and leptoquark internal lines to be less than the experimental uncertainty of $8 \times 10^{-12}$ gives $\operatorname{Re}\left(g_{R} g_{L}^{*}\right) /\left(M_{\mathrm{LQ}} / \mathrm{TeV}\right)^{2}<0.1$, and therefore the limits obtainable from the asymmetry $A_{2}$ are clearly more stringent. Conversely, the direct limit obtainable from $A_{1}$, viz., $\operatorname{Im}\left(g_{R} g_{L}^{*}\right)<2.5 \times 10^{-2}$ (for $M_{\mathrm{LQ}}=1 \mathrm{TeV}$ ) is not competitive with the indirect constraint derived from the experimental limit on the electric dipole moment $d_{e}$ of about $10^{-27}$ e cm, which leads to $\operatorname{Im}\left(g_{R} g_{L}^{*}\right) /\left(M_{\mathrm{LQ}} / \mathrm{TeV}\right)^{2}<10^{-6}$.

In conclusion, azimuthal asymmetries single out chirality-violating couplings in scalarleptoquark models. Longitudinal beam polarization can only put limits on the absolute values of the left and right chiral couplings. In the considered example the limit that can be put on the real part of the product of the couplings $g_{R} g_{L}^{*}$ is about four times better than the indirect limit from the $g_{e}-2$ of the electron.

Quantitative examples: The CP-conserving asymmetry provides a 4 times better limit for the chirality-violating couplings than the indirect most stringent limit coming from magnetic dipole moments. The CP-violating asymmetry could also directly probe $\mathrm{CP}$-violating phases in the leptoquark sector, however, indirect limits from the electron electric dipole moment are superior.

\subsection{Models of gravity in extra dimensions}

\subsubsection{Direct graviton production}

A signature for direct graviton production, envisaged in formulations of gravity with extra spatial dimensions, is a relatively soft photon and missing energy. The major background process is $\gamma \nu \bar{\nu}$ production. It is possible to determine the fundamental mass scale of gravity as well as the number of extra dimensions independently. Background suppression with right-handed electrons and left-handed positrons is extremely important, and the discovery reach is significantly enhanced.

Scenarios of gravity in extra spatial dimensions are currently being considered with great attention in the context of the hierarchy problem between the Planck and the Fermi mass scales [156]. The basic idea of the scenario of Arkani-Hamed, Dimopoulos and Dvali (ADD), is that only gravity can propagate in a bulk with $3+d(d \geq 2)$ spatial dimensions compactified to a radius $R$, while SM particles live in the usual four-dimensional space. The corresponding fundamental mass scale $M_{D}$, related to the four-dimensional Planck mass scale by

$$
G_{N}^{-1}=8 \pi R^{d} M_{D}^{2+d}
$$

with $G_{N}$ the Newton constant, can for $R$ of the sub-millimeter size be of the TeV order hence in the sensitivity reach of highest energy colliders.

In the usual four-dimensional space, the characteristic manifestation of the massless graviton propagating in $4+d$ extra dimensions is represented by the propagation (and emission) of a tower of massive Kaluza-Klein (KK) graviton excitation states. Accordingly, KK gravitons behave as massive, neutral particles very weakly (gravitationally) interacting with SM particles, their emission at colliders would typically be signalled by events with large missing transverse energy. The emission process is calculable in terms of an effective theory, where the number of extra dimensions $d$ and the fundamental gravitational mass scale $M_{D}$ appear as parameters $[157,158]$. Such a 'low-energy' effective in- 
teraction can give reliable results only for c.m. energy smaller than $M_{D}$, depending on the actual value of $d^{\ddagger}$., and is not supposed to be able to predict the model-dependent, and unknown, physical effects at scales above $M_{D}$. These effects should be related to uncalculated, higher dimensional, operators correcting the effective theory, and might mimic graviton emission signals.

At the linear collider, a candidate reaction for directly producing gravitons is $e^{+} e^{-} \rightarrow$ $\gamma G$. The differential cross section is $\left(x_{\gamma}=2 E_{\gamma} / \sqrt{s}, z=\cos \theta\right)$ :

$$
\frac{d \sigma}{d x_{\gamma} d z}=\frac{\alpha}{64 s} \frac{2 \pi^{d / 2}}{\Gamma(d / 2)}\left(\frac{\sqrt{s}}{M_{D}}\right)^{d+2} x_{\gamma}\left(1-x_{\gamma}\right)^{d / 2-1} F_{1}\left(x_{\gamma}, z\right),
$$

where the function $F_{1}$ is explicitly given in [157]. By its power-dependence on the ratio $\sqrt{s} / M_{D}$, eq. (3.36) clearly shows its effective-interaction character: at fixed $d$ the cross section increases with the c.m. energy and decreases for larger $M_{D}$ while, recalling that $\sqrt{s} / M_{D}$ must be smaller than unity for the effective-theory to be reliable, larger $d$-values result in smaller cross sections, hence in decreasing statistics. Also, eq. (3.36) shows that for $d>2$ the photon spectrum tends to concentrate towards small energy, so that relatively 'soft' photons should be expected.

The major SM background is determined by $e^{+} e^{-} \rightarrow \gamma \nu \bar{\nu}$. The contribution from $e^{+} e^{-} \rightarrow \gamma Z \rightarrow \gamma \nu \bar{\nu}$ can easily be eliminated by cutting out the $E_{\gamma}$ region around the corresponding $Z$-peak, but there remains a significant, continuous, distribution in $E_{\gamma}$ from $e^{+} e^{-} \rightarrow \gamma \nu \bar{\nu}$ that has similar behaviour as the signal. This part of the background must therefore be calculated by appropriate simulation codes and subtracted in order to use the eventual excess single-photon cross sections to derive information on $d$ and $M_{D}$. In this regard, since the neutrino coupling is only left-handed, the background has nearly maximal polarization asymmetry and, consequently, polarized electron and positron beams should be extremely effective in suppressing the $\gamma \nu \bar{\nu}$ channel.

An example is presented in fig. 3.27 which shows, for different $d$ and $M_{D}$, the signal $e^{+} e^{-} \rightarrow \gamma G$ cross section at $\sqrt{s}=800 \mathrm{GeV}$, taking into account also various acceptance cuts, ISR and beamstrahlung effects [160]. The background $\gamma \nu \bar{\nu}$ for various choices of $e^{+}$ and $e^{-}$polarizations is also reported, and the comparison with the signal clearly shows the strong reduction power obtainable from beam polarization, such that the LC potential for exploring the graviton emission is greatly enhanced.

The corresponding 5- $\sigma$ discovery reach on the fundamental gravitational scale $M_{D}$ for various numbers of extra dimensions, at $\sqrt{s}=800 \mathrm{GeV}$ and $\mathcal{L}_{\text {int }}=1 \mathrm{ab}^{-1}$, is shown for both unpolarized and polarized beams in table 3.9 [160]. Here the benefits of high degree initial polarizations are quite evident.

If extra dimensions are the origin of the anomalous single-photon rates, a determination of the number of extra dimensions could in principle be attempted by measuring the excess (polarized) cross sections at different c.m. energies and making a fit to the expected behaviour $\sigma \sim(\sqrt{s})^{d}$; some numerical estimates of the sensitivity are presented in ref. [160]. Hopefully, the result for $d$ should be an integer number in order to interpret excess events as graviton emission. In conclusion, studying single-photon plus missing

\footnotetext{
${ }^{\ddagger}$ Numerous alternative model realizations of gravity in extra dimensions exist, with different features from the ADD regarding, e.g., the KK graviton spectrum, the $R$ and $d$-admitted values, and even the possibility that also SM matter could live in higher-dimensional spaces. For a phenomenological review of models and the current experimental situation, see e.g. [159]
} 


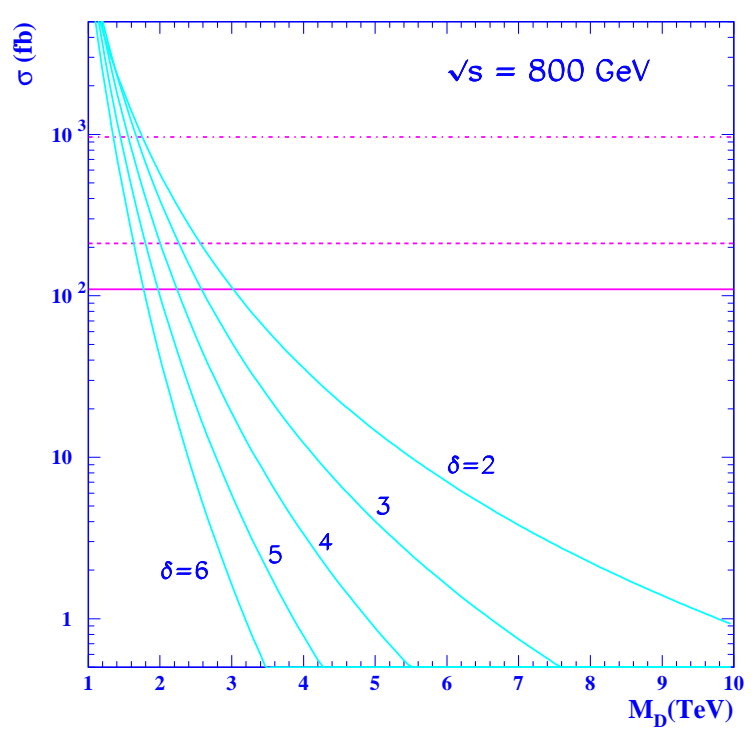

Figure 3.27: Total cross sections for $e^{+} e^{-} \rightarrow \gamma G$ at $\sqrt{s}=800 \mathrm{GeV}$ as a function of the scale $M_{D}$ and of the number $d$ of extra dimensions. The cross section is for $\left(P_{e^{-}}, P_{e^{+}}\right)=(80 \%,-60 \%)$. The three horizontal lines indicate the background cross sections from $e^{+} e^{-} \rightarrow \nu \bar{\nu} \gamma$ for both beams polarized (solid), only electron beam polarized (dashed) and no polarization (dot-dashed). The signal cross sections are reduced by a factor of 1.48 for the latter two scenarios [160].

\begin{tabular}{|r|c|c|c|}
\hline$M_{D}[\mathrm{TeV}]$ & $\left(\left|P_{e^{-}}\right|,\left|P_{e^{+}}\right|\right)=(0,0)$ & $\left(\left|P_{e^{-}}\right|,\left|P_{e^{+}}\right|\right)=(80 \%, 0)$ & $\left(\left|P_{e^{-}}\right|,\left|P_{e^{+}}\right|\right)=(80 \%,-60 \%)$ \\
\hline$d=2$ & 4.48 & 6.27 & 7.86 \\
3 & 3.54 & 4.63 & 5.55 \\
4 & 2.91 & 3.64 & 4.23 \\
5 & 2.47 & 3.00 & 3.41 \\
\hline
\end{tabular}

Table 3.9: Discovery (5- $\sigma$ ) reach $M_{D}$ for direct graviton production $e^{+} e^{-} \rightarrow \gamma G$ with $\mathcal{L}_{\text {int }}=$ $1 \mathrm{ab}^{-1}$ at $\sqrt{s}=800 \mathrm{GeV}$, for various numbers of extra dimensions $d$ [160]. The major background, $e^{+} e^{-} \rightarrow \nu \bar{\nu} \gamma$, can be efficiently suppressed with beam polarization.

energy events at the LC with different c.m. energies and beam polarizations can be essential not only for model-independent tests of gravity in extra dimensions, but also to help distinguishing graviton emission signals from effects of higher dimensional operators.

Studies of graviton emission can also be performed at hadron colliders, e.g., at the LHC the leading experimental signal is expected from inclusive $p p \rightarrow$ jet $+E_{\mathrm{T}}$ from $q g \rightarrow q G, q \bar{q} \rightarrow g G$ and $g g \rightarrow g G$. The situation in this case is complicated by the fact that the parton c.m. sub-energy $\sqrt{\hat{s}}$ can take different values, and the elementary production sub-processes may well-occur in regions where $\hat{s} \gg M_{D}^{2}$ and the 'low-energy' effective-theory approach is not applicable to predict the excess 'monojet' cross sections. ${ }^{\S}$ Thus, on the one hand $M_{D}$ must be not too small, and on the other hand $d$ must be not

\footnotetext{
${ }^{\S}$ At the LC with foreseen c.m. energies in the TeV region, the condition $\sqrt{s} / M_{D}<1$ assuring the applicability of the effective-theory is, conversely, naturally fulfilled.
} 
too large. In practice a range in $d$ and $M_{D}$ must be pre-determined phenomenologically, by truncation procedures in $\hat{s}$ of the parton-model cross sections and cuts on other kinematical variables. Also, initial polarization is not available for background suppression. Typically, the $M_{D}$ ranges in which a $5 \sigma$ discovery potential may be achieved at the LHC with $\mathcal{L}_{\text {int }}=100 \mathrm{fb}^{-1}$, are (in TeV): $4.0-7.5,4.5-5.9$ and $5-5.3$ for $d=2,3$ and 4 , respectively [161]. For higher values of $d$ the effective theory may break down, whereas they may still be in the reach of the ILC.

One can conclude, from all the considerations exposed above, that the LC with polarized beams has a really important role, also as a complement to LHC, in searches and tests of gravity in extra dimensions.

Quantitative examples: The detection of direct graviton signals critically depends on the suppression of the dominant background process $\gamma \nu \bar{\nu}$. Compared with the case of only electrons polarized, the background process is suppressed by a factor of about 2 with $60 \%$ positron polarization whereas the signal is enhanced by a factor of about 1.5 . Furthermore, the discovery reach is enhanced by an amount depending on the number of extra dimensions, for example by about $25 \%(14 \%)$ for $d=2(d=5)$.

\subsubsection{Signatures of extra dimensions in fermion pair production}

Indirect signals of TeV-scale gravity propagating in large, compactified, extra spatial dimensions can be probed in the framework of effective contact interactions. With both beams longitudinally polarized, the reach on the relevant mass scales and their identification over indirect effects from different kinds of non-standard interactions, can be improved by $10-15 \%$ with respect to the cases of no polarization or only electron polarization. Furthermore, transverse beam polarization allows an unambiguous distinction between different realizations of extra-dimensional space-time.

Two typical model examples of the ADD [156] and Randall-Sundrum (RS) [162] scenarios are discussed in the following.

In the ADD model, the exchanged tower of KK graviton excitations has an evenly spaced, and almost continuous, mass spectrum with steps $\Delta m \propto 1 / R$. The summation over the KK states results in an effective $D=8$ graviton-exchange interaction, which can be written in the notation of [163]:

$$
\mathcal{L}^{\mathrm{ADD}}=i \frac{4 \lambda}{M_{H}^{4}} T^{\mu \nu} T_{\mu \nu},
$$

with $T_{\mu \nu}$ the stress-energy tensor of SM particles, $M_{H}$ a cut-off on the summation over the KK spectrum expected of the TeV order, and $\lambda= \pm 1$. The exchange of spin-2 graviton fields introduces, in the helicity amplitudes for $e^{+} e^{-} \rightarrow f \bar{f}$, additional terms with new and characteristic angular dependences $[163,164]$. Such deviations can be parametrized phenomenologically by the coupling:

$$
f_{G}=\frac{\lambda s^{2}}{4 \pi \alpha_{\mathrm{em}} M_{H}^{4}} .
$$

The (high) dimensionality $D=8$ of (3.37) implies the suppression of deviations from the SM cross section originating from graviton-exchange by the large power $\left(\sqrt{s} / M_{H}\right)^{4}$. This should be compared to the case of the four-fermion contact-interaction scales $\Lambda$, where 
the effects are suppressed by only $(\sqrt{s} / \Lambda)^{2}$, so that numerically lower bounds on $M_{H}$ can be expected on statistical grounds. Indeed, the search reach on $M_{H}$ scales, according to previous arguments, as

$$
M_{H} \sim\left[s^{(d-5)} \mathcal{L}_{\text {int }}\right]^{1 /(2 d-8)}=\left(s^{3} \cdot \mathcal{L}_{\text {int }}\right)^{1 / 8} .
$$

In the simplest version of the RS scenario [162,165] the setting is a five-dimensional space-time, the KK excitations are not equally spaced and the characteristic feature is the existence of a spectrum of narrow spin-2 resonance states with masses expected in the $\mathrm{TeV}$ range. Formally, this can be obtained from (3.37) by the replacement

$$
\frac{\lambda}{M_{H}^{4}} \rightarrow \frac{-1}{8 \Lambda_{\pi}^{2}} \sum_{n} \frac{1}{s-m_{n}^{2}+i m_{n} \Gamma_{n}}
$$

where $\Lambda_{\pi}$ is of the TeV order and $m_{n}\left(\Gamma_{n}\right)$ are the masses (widths) of the TeV scale KK excitations. (In the applications presented here, widths are neglected in the evaluation of the relevant cross sections [166].)

The spin-2 nature of these graviton exchanges can be explicitly verified through the direct analysis of the resonances themselves, were they actually produced. Conversely, with the linear collider energy below the production threshold, the indirect signals of $s$ channel exchange of such massive graviton fields through deviations of the $e^{+} e^{-} \rightarrow f \bar{f}$ cross sections from the SM predictions can be tested, and distinguished by appropriately defined observables, from different possible new physics sources of deviations, such as the 'conventional' 4-fermion contact interactions [138, 142,145].

\section{Identification of graviton-exchange effects}

The discovery and the identification reaches on, respectively, $\Lambda$ and $M_{H}$ can be assessed by analysing either the dependence of cross sections on the polar angle, or some suitably defined asymmetries among integrated differential distributions. The sensitivity to newphysics effects can be described by

a) the discovery reach, which gives values for $\Lambda$ or $M_{H}$ up to which a deviation from the Standard Model predictions can be observed, and

b) the identification reach corresponding to values for $\Lambda$ or $M_{H}$ up to which the particular models producing the deviation can be differentiated from each other.

The identification reach at the ILC on $M_{H}$ at 5- $\sigma$ from $e^{+} e^{-} \rightarrow f \bar{f}$ is shown in the left panel of table 3.10, for different luminosities and longitudinal polarization configurations. Systematic uncertainties on luminosity and beam polarization, in addition to the statistical ones, have been taken into account.

A clear signature of graviton exchange arises in the differential angular distributions, in the left-right asymmetry and in the center-edge asymmetries (see, e.g., [144, 163, 166, 167]). In the left panel of table 3.10, the values of $M_{H}$, indicated as a $5 \sigma$ identification reach, represent the resolution power for distinguishing signals of graviton exchange (3.38) from effects of contact interactions (3.25). The results include the combination of the channels $f=\mu, \tau, b, c$ and are a good example of the benefits of positron longitudinal polarization. 


\subsubsection{Use of transversely-polarized beams for graviton searches}

If both beams are transversely polarized, additional azimuthal asymmetries sensitive to spin-2 graviton exchange can be defined. Although the identification reach on the scale $M_{H}$ is similar to that obtained from just longitudinal electron and positron polarization, the specific, azimuthal asymmetries allow an unambiguous separation of the indirect manifestations of ADD- and RS-like extra-dimensional models.

With reference to the Introduction, eq. (1.14), the $\phi$-dependent part of the matrix element squared for $e^{+} e^{-} \rightarrow f \bar{f}$ will now be considered. In the study reviewed here only terms bilinear in the transverse polarization contribute, and the two transverse polarization vectors are assumed to be antiparallel. In the observables considered in the previous subsection, this contribution to the differential cross section has been eliminated by the integration over the full range of $\phi$.

The following azimuthal asymmetry can be defined [169]:

$$
\frac{1}{N} \frac{d A^{T}}{d \cos \theta}=\frac{1}{\sigma}\left[\int_{+} \frac{d \sigma}{d \cos \theta d \phi}-\int_{-} \frac{d \sigma}{d \cos \theta d \phi}\right],
$$

where $\theta$ denotes the polar angle and $\int_{ \pm}$indicates integrations over regions where $\cos 2 \phi$ takes on positive or negative values. Examples of the $z=\cos \theta$ dependence of the expression (3.41) in the SM and in the ADD scenario are shown in fig. 3.28, for final states and transverse polarization configurations as described in the caption.

From eq. (3.41), the azimuthal forward-backward asymmetry, sensitive to $\cos \theta$-odd terms can be defined:

$$
A_{\mathrm{FB}}^{T}=\frac{1}{N}\left[\int_{\cos \theta \geq 0}-\int_{\cos \theta \leq 0}\right] d \cos \theta \frac{d A^{T}}{d \cos \theta} .
$$

For both for the SM and the 4-fermion contact-interactions one finds $A_{\mathrm{FB}}^{T}=0$, since $N^{-1} d A^{T} / d \cos \theta \propto 1-\cos ^{2} \theta$ is even in $\cos \theta$ in these cases. Conversely, the spin- 2 graviton exchange introduces $\cos \theta$-odd terms, so that $A_{\mathrm{FB}}^{T}$ is non-zero for this kind of new physics that, accordingly, can be identified by this observable. The corresponding expected identification reach on $M_{H}$ is reported in the right panel of table 3.10 [169]. Concerning the values of transverse polarizations, $100 \%$ efficiency of the spin rotators have been assumed.

Actually, as an alternative to $A_{\mathrm{FB}}^{T}$, the distinction of graviton exchange from other newphysics effects might be attempted by a direct fit to the $\cos \theta$ dependence of $N^{-1} d A^{T} / d \cos \theta$, emphasizing the deviations from the $1-\cos ^{2} \theta$ behaviour. Examples for the reach and for the identification reach on $M_{H}$, the latter being potentially as high as $10 \sqrt{s}$, are shown in the left panel of table 3.11, up to very high energies. It gives the values of $M_{H}$ corresponding to deviations from the SM-predicted angular behaviour that could be observed on statistical grounds. However, notice that for high luminosities, those values are expected to be reduced by the systematic effects, which become dominant.

\section{Distinguishing among models with graviton exchange}

In the previous subsection, identification reaches on graviton exchange mass scales from azimuthal asymmetries allowed by transverse beam polarization are found numerically 
equivalent to those obtained by longitudinally-polarized beams. The point now is the distinction between different extra-dimensional scenarios, in the specific chosen examples, the ADD and the RS scenarios, were indirect signals of these mechanisms observed.

In the RS model, if $\sqrt{s}$ is far from the $Z$ and the $K K$ poles, the imaginary part of the amplitude entering the $\sin 2 \phi$ term in the differential cross section with transverselypolarized beams becomes vanishingly small. Conversely, the summation over the essentially continuous spectrum of ADD gravitons can lead to a finite, cut-off independent imaginary part, also sensitive to the number of extra dimensions [168]. Consequently, $f_{G}$ of eq. (3.38) can acquire an imaginary part, strongly dependent on the number of extra dimensions.

With transverse polarization one can define a new asymmetry sensitive to such an imaginary part, that exploits the $\sin 2 \phi$ term:

$$
\frac{1}{N} \frac{d A_{i}^{T}}{d \cos \theta}=\frac{1}{\sigma}\left[\int_{+} \frac{d \sigma}{d \cos \theta d \phi}-\int_{-} \frac{d \sigma}{d \cos \theta d \phi}\right],
$$

where now $\int_{ \pm}$indicate integrations over regions of positive and negative values of $\sin 2 \phi$. It can be seen that the $\cos 2 \phi$ terms proportional to the real part cancel for both the RS and ADD models. The observable (3.43) vanishes identically for both the SM and the RS scenario, thus a non-zero value unambiguously signals ADD graviton exchange. Fig. 3.29 exemplifies the $\cos \theta$ behaviour for selected values of the ADD mass scales. The right panel of table 3.11 reports the 5- $\sigma$ discovery reach from the asymmetry (3.43), assuming $d=3$ for illustration purposes, and indicates that the indirect manifestations of the ADD and RS models could be distinguished from one another up to about $M_{H} \sim(2.5-3.0) \sqrt{s}$ [169]. This shows the essential role of transverse beam polarization in this kind of analysis, which otherwise could not be performed.
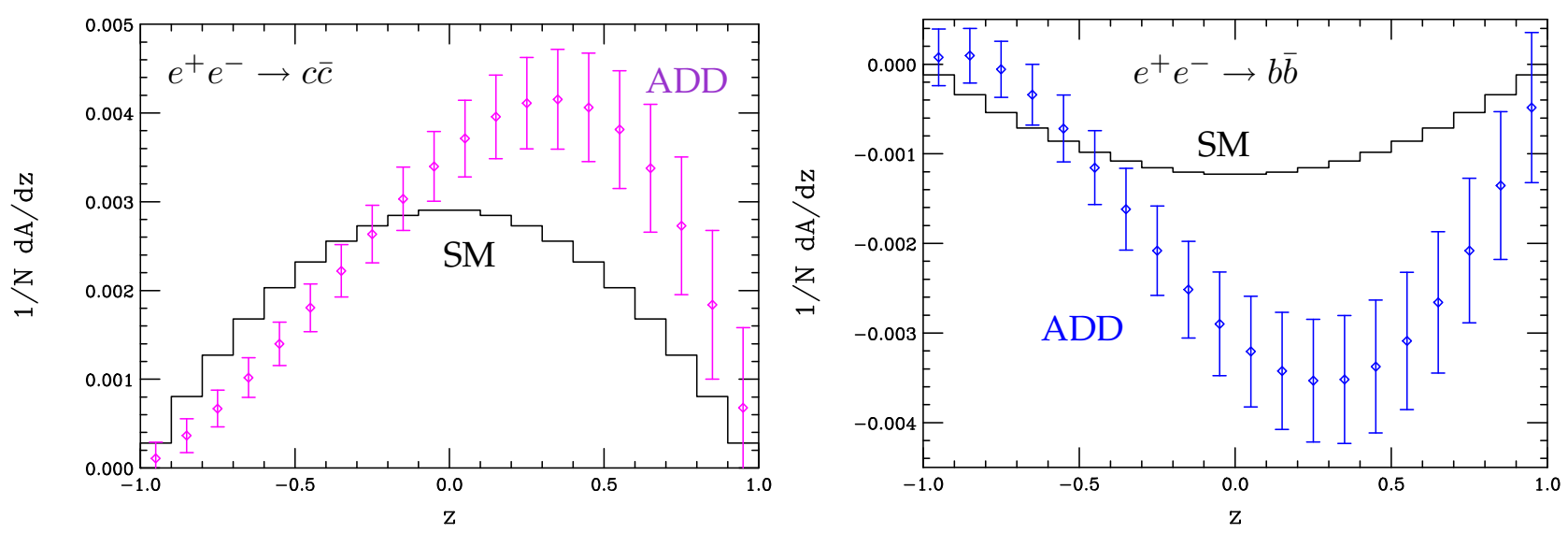

Figure 3.28: Differential azimuthal asymmetry distribution for $e^{+} e^{-} \rightarrow f \bar{f}$, i.e. $c \bar{c}$ (left) and $b \bar{b}$ (right), at a $500 \mathrm{GeV} \mathrm{LC} \mathrm{assuming} \mathrm{a} \mathrm{luminosity} \mathrm{of} 500 \mathrm{fb}^{-1}, z=\cos \theta$. The histograms are the SM predictions while the data points assume the ADD model with $M_{H}=1.5 \mathrm{TeV} ;\left(P_{e^{-}}^{\mathrm{T}}, P_{e^{+}}^{\mathrm{T}}\right)=(80 \%, 60 \%)$ [169].

Quantitative example: With transversely-polarized beams a new asymmetry can be built, which is sensitive to imaginary parts of the graviton propagator. Below the graviton poles no imaginary parts occur in the RS model, whereas in the ADD model a cut-off independent imaginary part emerges. This new observable allows a distinction between the two models in LC studies up to a scale $M_{H} \sim 3 \sqrt{s}$. 


$M_{H}[\mathrm{TeV}], \sqrt{s}=0.5 \mathrm{TeV}$, long. pol.
\begin{tabular}{|c||c|c|c|}
\hline \multicolumn{1}{|c||}{} & \multicolumn{4}{c|}{$\mathcal{L}_{\text {int }}\left[\mathrm{fb}^{-1}\right]$} \\
$\left(P_{e^{-}}, P_{e^{+}}\right)$ & 100 & 300 & 500 \\
\hline \hline$(0,0)$ & 2.3 & 2.6 & 2.9 \\
\hline$(+80 \%, 0)$ & 2.5 & 2.8 & 3.05 \\
\hline$(+80 \%,-60 \%)$ & 2.45 & 3.0 & 3.25 \\
\hline
\end{tabular}

\begin{tabular}{|c||c|c|c|c|}
\multicolumn{1}{|c|}{$M_{H}[\mathrm{TeV}],\left(P_{e^{-}}^{\mathrm{T}}, P_{e^{+}}^{\mathrm{T}}\right)=(80 \%, 60 \%)$} \\
\hline & 100 & 300 & 500 & 1000 \\
\hline \multicolumn{1}{|c||}{$\mathcal{L}_{\text {int }}$} & $\left.\mathrm{fb}^{-1}\right]$ \\
\hline$\sqrt{s}=0.5 \mathrm{TeV}$ & 1.6 & 1.9 & 2.0 & 2.2 \\
\hline$\sqrt{s}=0.8 \mathrm{TeV}$ & 2.4 & 2.6 & 2.8 & 3.1 \\
\hline$\sqrt{s}=1.0 \mathrm{TeV}$ & 2.8 & 3.2 & 3.4 & 3.8 \\
\hline
\end{tabular}

Table 3.10: Left: 5- $\sigma$ identification reach on the mass scale $M_{H}$ vs. integrated luminosity from the process $e^{+} e^{-} \rightarrow f \bar{f}$, with $f$ summed over $\mu, \tau, b, c$, and for the energy $0.5 \mathrm{TeV}$ [167] (see also [144], where the 95\% C.L. sensitivity for $M_{H}$ in $e^{+} e^{-} \rightarrow \mu \bar{\mu}, c \bar{c}, b \bar{b}$ has been simulated). Right: 5- $\sigma$ identification reach in $M_{H}$ vs. integrated luminosity using $A_{\mathrm{FB}}^{T}$ for the process $e^{+} e^{-} \rightarrow f \bar{f}$, with $f$ summed over $\mu, \tau, b, c$ and $t$ [169]. .

\begin{tabular}{|c|c||c|}
\hline $\begin{array}{c}E_{\mathrm{CM}} \\
{[\mathrm{GeV}]}\end{array}$ & $\begin{array}{c}\text { Ident. reach } \\
M_{H}[\mathrm{TeV}]\end{array}$ & $\begin{array}{c}95 \% \text { CL disc. reach } \\
M_{H}[\mathrm{TeV}]\end{array}$ \\
\hline \hline 500 & 5.4 & 10.2 \\
\hline 800 & 8.8 & 17.0 \\
\hline 1000 & 11.1 & 21.5 \\
\hline 1200 & 13.3 & 26.0 \\
\hline 1500 & 16.7 & 32.7 \\
\hline
\end{tabular}

\begin{tabular}{|c||c|c|c|c|}
\hline \multicolumn{1}{|c||}{ 5- $\sigma$ disc. reach } & \multicolumn{4}{c|}{$\mathcal{L}_{\text {int }}\left[\mathrm{fb}^{-1}\right]$} \\
$M_{H}[\mathrm{TeV}]$ & 100 & 300 & 500 & 1000 \\
\hline \hline$\sqrt{s}=0.5 \mathrm{TeV}$ & 1.2 & 1.3 & 1.4 & 1.6 \\
\hline$\sqrt{s}=0.8 \mathrm{TeV}$ & 1.8 & 2.0 & 2.2 & 2.4 \\
\hline$\sqrt{s}=1.0 \mathrm{TeV}$ & 2.2 & 2.4 & 2.6 & 2.8 \\
\hline
\end{tabular}

Table 3.11: Left: Identification reach and discovery reach $M_{H}$ in the ADD model by fitting the distribution $N^{-1} d A^{T} / d \cos \theta \sim 1-\cos ^{2} \theta$ for the final states $f=\mu, \tau, f=b$ and $f=c$ for different centre-of-mass energies. Right: $5-\sigma$ reach for the discovery of a non-zero value of the azimuthal asymmetry (3.43) vs. $\mathcal{L}_{\text {int }}$ for $d=3 ; M_{H}=M_{D}$ is assumed throughout as is $\left(P_{e^{-}}^{\mathrm{T}}, P_{e^{+}}^{\mathrm{T}}\right)=(80 \%, 60 \%)[169]$.

\subsection{Other mechanisms of CP violation}

\subsubsection{Unconventional interactions in $t \bar{t}$ production}

(Pseudo-) scalar or tensor interactions associated with a new physics scale $\Lambda$ can lead to CP-odd observables from interference terms with the virtual $\gamma, Z$ exchange in the annihilation channel. Only with both beams transversely polarized are these $\mathrm{CP}$-violating effects measurable without final-state spin analysis.

The observation of $\mathrm{CP}$ violation in $e^{+} e^{-}$collisions requires either the measurement of the polarization of the final-state particles or the availability of polarized beams. Transverse polarization of initial beams defines one more direction, and can provide CP-odd asymmetries without the need for directly measuring final-state polarizations. This may represent an advantage, e.g. as regards the statistical significance of the signal.

Transverse polarization (TP) enables novel CP violation searches in the inclusive process [12]

$$
e^{+} e^{-} \rightarrow A+X
$$

As regards the search for non-standard sources of CP violation, when the spin of particle $A$ is not observed and $m_{e}$ is neglected, only (pseudo-) scalar or tensor currents associated with a new-physics scale $\Lambda$ can lead to CP-odd observables at the leading order 


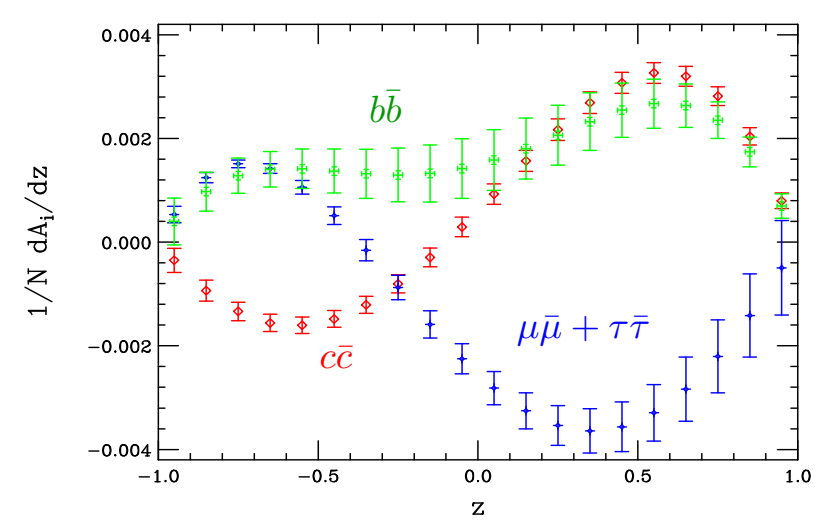

Figure 3.29: The $N^{-1} d A_{i}^{T} / d z$ distributions at $\sqrt{s}=500 \mathrm{GeV}$ assuming $M_{H}=M_{D}=1.5$ $\mathrm{TeV}$ and $d=3$ with an integrated luminosity of $500 \mathrm{fb}^{-1}$. The plotted points from top to bottom in the centre of the plot correspond, respectively, to $f=b, \mu$ and $\tau$ combined together, and $c$ [169].

in the new interaction if transversely-polarized beams are used. They are due to the interference between these new currents and the $\gamma$ and $Z$ exchanges in the $s$-channel, cf. also the matrix element squared in the general expression, eq. (1.14). Contributions from (pseudo-) scalar or tensor currents lead to interference terms which are linear in the transverse polarizations. However, to construct CP-odd observables both beams must be transversely polarized: one must compare contributions with transversely-polarized electrons with those resulting from transversely-polarized positrons (with the same polarization degree). Vector-like four-fermion contact interactions can interfere with the SM amplitudes, but for this kind of (helicity-conserving) non-standard interactions no CPodd distribution can arise even in the presence of TP. Thus, the above-mentioned CP-odd observables have a great selective power of novel (helicity-changing) interactions at the first order. With longitudinal beam polarization there would be no interference of the SM with scalar or tensor interactions.

In the following the contributions to the differential cross section due to (pseudo-) scalar and tensor contact-interactions at leading order in the interaction strengths for the process $e^{+} e^{-} \rightarrow t \bar{t}$ [149] are reviewed. This allows to construct an effective up-down asymmetry and a version of the same integrated over the polar angle.

An effective Lagrangian that can parametrize the above-mentioned non-standard interactions in a general, model-independent way can be written as:

$$
\mathcal{L}=\mathcal{L}^{\mathrm{SM}}+\frac{1}{\Lambda^{2}} \sum_{i}\left(\alpha_{i} \mathcal{O}_{i}+\text { h.c. }\right)
$$

where $\alpha_{i}$ are the coefficients which parametrize non-standard interactions and are assumed to be of order unity, $\mathcal{O}_{i}$ are the possible effective dimension-six operators, and $\Lambda$ is the scale of new physics.

After Fierz transformation the part of the Lagrangian containing the above four-Fermi operators, and relevant to $e^{+} e^{-} \rightarrow t \bar{t}$, eq. (3.45) can be rewritten as

$$
\mathcal{L}^{4 F}=\sum_{i, j=L, R}\left[S_{i j}\left(\bar{e} P_{i} e\right)\left(\bar{t} P_{j} t\right)+V_{i j}\left(\bar{e} \gamma_{\mu} P_{i} e\right)\left(\bar{t} \gamma^{\mu} P_{j} t\right)+T_{i j}\left(\bar{e} \frac{\sigma_{\mu \nu}}{\sqrt{2}} P_{i} e\right)\left(\bar{t} \frac{\sigma^{\mu \nu}}{\sqrt{2}} P_{j} t\right)\right]+\text { h.c. }
$$


where $P_{\mathrm{L}, \mathrm{R}}$ are chirality projection operators and the coefficients must satisfy the relations

$$
S_{\mathrm{RR}}=S_{\mathrm{LL}}^{*}, \quad S_{\mathrm{LR}}=S_{\mathrm{RL}}=0, \quad V_{i j}=V_{i j}^{*}, \quad T_{\mathrm{RR}}=T_{\mathrm{LL}}^{*}, \quad T_{\mathrm{LR}}=T_{\mathrm{RL}}=0 .
$$

The differential cross section depends on the combined couplings

$$
S \equiv S_{\mathrm{RR}}+\frac{2 c_{A}^{t} c_{V}^{e}}{c_{V}^{t} c_{A}^{e}} T_{\mathrm{RR}}
$$

where $c_{V}^{e, t}, c_{A}^{e, t}$ are the couplings of $Z$ to $e^{+} e^{-}$and $t \bar{t}$. In (3.48) the contribution of the tensor term relative to the scalar term is suppressed by a factor $2 c_{A}^{t} c_{V}^{e} / c_{V}^{t} c_{A}^{e} \approx 0.36$. In what follows only the combination $S$ is considered, not $S_{\mathrm{RR}}$ and $T_{\mathrm{RR}}$ separately.

One can construct a CP-odd asymmetry, up-down asymmetry, as

$$
A(\theta)=\frac{\int_{0}^{\pi} \frac{d \sigma^{+-}}{d \Omega} d \phi-\int_{\pi}^{2 \pi} \frac{d \sigma^{+-}}{d \Omega} d \phi}{\int_{0}^{\pi} \frac{d \sigma^{+-}}{d \Omega} d \phi+\int_{\pi}^{2 \pi} \frac{d \sigma^{+-}}{d \Omega} d \phi},
$$

where the superscripts denote opposite transverse polarization of $e^{-}, e^{+}$and $\phi$ is the azimuthal angle. One can see that $A(\theta)$ is proportional to the imaginary part of $S$ [149], that appears in the differential distribution as a factor of the $\sin \theta \sin \phi$ term.

Also a $\theta$-integrated version of the asymmetry can be defined,

$$
A\left(\theta_{0}\right)=\frac{1}{\sigma^{+-}\left(\theta_{0}\right)} \int_{-\cos \theta_{0}}^{\cos \theta_{0}}\left[\int_{0}^{\pi}-\int_{\pi}^{2 \pi}\right] \frac{d \sigma^{+-}}{d \Omega} d \cos \theta d \phi,
$$

where a cut-off angle $\theta_{0}$ has been introduced to be away from the beam-pipe direction and $\sigma^{+-}\left(\theta_{0}\right)$ is the cross section integrated with this cut-off.

In a numerical study, limits on $S$ can be put using the integrated asymmetry $A\left(\theta_{0}\right)$ and optimized by tuning the value of $\theta_{0}$.
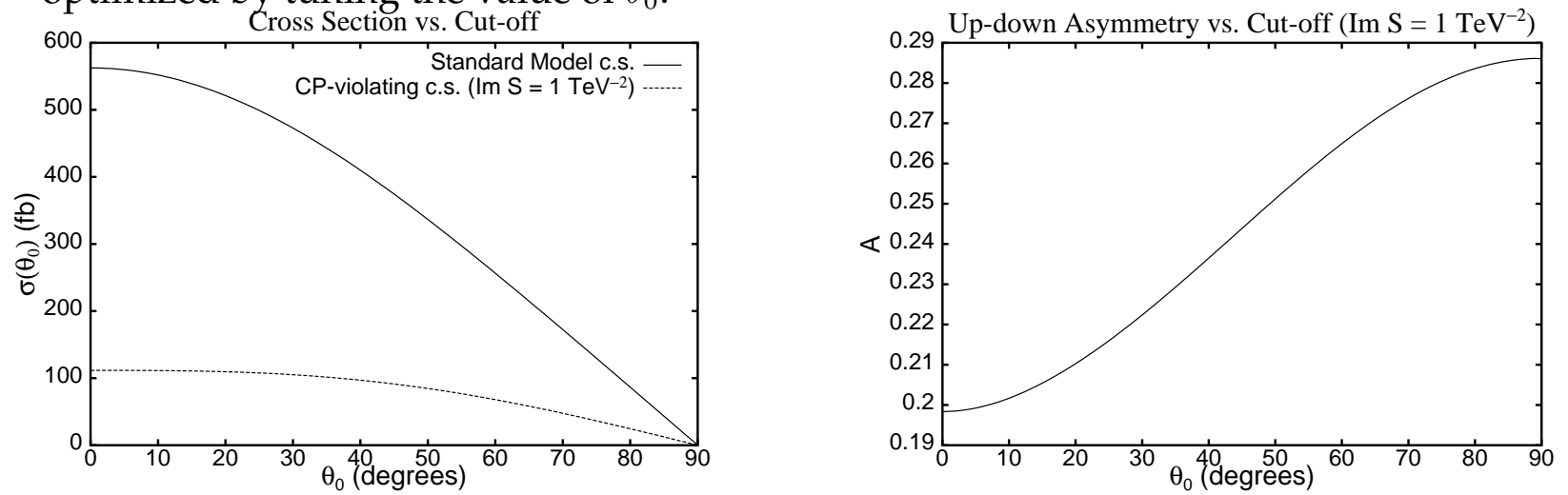

Figure 3.30: Left: The SM cross section (solid line) and the numerator of the asymmetry $A\left(\theta_{0}\right)$ in eq. (3.50) (broken line) vs. $\theta_{0}$; Right: The asymmetry $A\left(\theta_{0}\right)$ defined in eq. (3.50) vs. $\theta_{0}$ for $\operatorname{Im} S=1 \mathrm{TeV}^{-2}[149]$.

As can be seen from fig. 3.30, the value of $A\left(\theta_{0}\right)$ increases with $\theta_{0}$, because the SM cross section in the denominator of eq. (3.50) decreases with this cut-off faster than the numerator. Here, and in the subsequent figure, $\sqrt{s}=500 \mathrm{GeV}$ and $\left(P_{e^{-}}^{\mathrm{T}}, P_{e^{+}}^{\mathrm{T}}\right)=(100 \%, 100 \%)$ are assumed.

IAn analogous up-down asymmetry which is available with transverse beam polarization allows for the separation of light $u$ and $d$-type flavours [170]. 


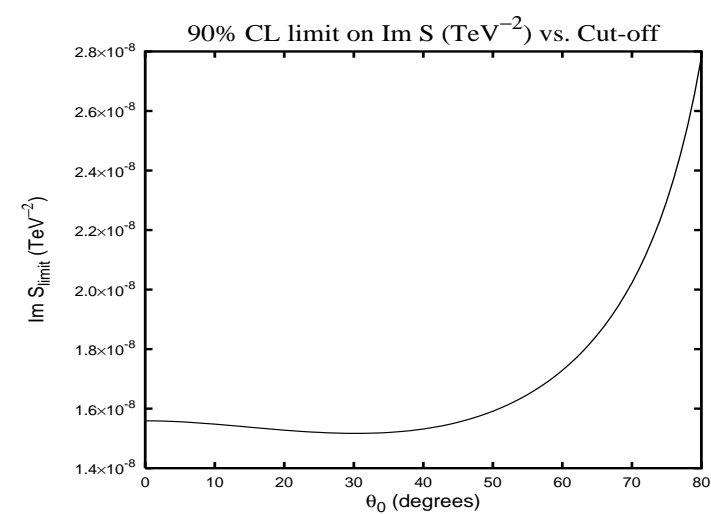

Figure 3.31: The 90\% C.L. that can be obtained on $\operatorname{Im} S$ with an integrated luminosity of $500 \mathrm{fb}^{-1}$ vs. the cut-off angle $\theta_{0}[149]$.

Fig. 3.31 shows the $90 \%$ confidence level (C.L.) limits that could be placed on $\operatorname{Im} S$ for an integrated luminosity of $\mathcal{L}_{\text {int }}=500 \mathrm{fb}^{-1}$. This limit translates to a value of $\Lambda$ of the order of $8 \mathrm{TeV}$, assuming that the coefficients $\alpha_{i}$ in (3.45) are of order 1 . The corresponding limit for $\sqrt{s}=800 \mathrm{GeV}$ with the same integrated luminosity and complete TP is $\sim 9.5 \mathrm{TeV}$.

Using the more realistic transverse polarization degrees of $80 \%$ and $60 \%$, the up-down asymmetry $A(\theta)$ or $A\left(\theta_{0}\right)$ gets multiplied by a factor $\frac{1}{2}\left(P_{e^{-}}^{\mathrm{T}}-P_{e^{+}}^{\mathrm{T}}\right)$. For $\left(P_{e^{-}}^{\mathrm{T}}, P_{e^{+}}^{\mathrm{T}}\right)=$ $(80 \%,-60 \%)$, this means a reduction of the asymmetry by a factor of 0.7 . Since the SM cross section does not change, this also means that the limit on the parameter $\operatorname{Im} S$ goes up by a factor of $1 / 0.7 \approx 1.4$, and the limit on $\Lambda$ goes down by a factor of $\sqrt{0.7} \approx 0.84$, to about $6.7 \mathrm{TeV}$. If the positron beam is unpolarized, however, the sensitivity decreases further.

In summary, TP can be used to study CP-violating asymmetries arising from the interference of new-physics scalar and tensor interactions with the $\operatorname{SM} \gamma$ - and $Z$-exchanges. These interference terms cannot be seen with longitudinally-polarized or unpolarized beams. Moreover, such an asymmetry would not be sensitive to new vector and axialvector interactions (as for example, from an extra $Z^{\prime}$ neutral boson), or even electric or "weak" dipole interactions of heavy particles, since it vanishes if $m_{e} \sim 0$. This manifests the selective power on new physics of this observable.

Quantitative example: With both beams transversely polarized, CP-odd asymmetries can be constructed which are sensitive to new CP-violating (pseudo-) scalar or tensor interactions. The corresponding new physics scale $\Lambda$ can be bounded at the $90 \%$ confidence level, at about $7-10 \mathrm{TeV}$, with plausible assumptions on the centre-of-mass energy and the values of transverse beam polarizations.

\subsubsection{Transversely-polarized beams for $\mathrm{CP}$ violation in $\gamma Z$ production}

An anomalous CP-violating $\gamma \gamma Z$ vertex gives rise to a novel asymmetry which is accessible with transversely-polarized beams in the process $e^{+} e^{-} \rightarrow \gamma Z$. This asymmetry, which is odd under naive time reversal, is proportional to the real part of the $\gamma \gamma Z$ $\mathrm{CP}$-violating coupling and is not accessible with longitudinally-polarized beams but could be measured with transversely-polarized beams. This is in contrast to the simple forward-backward asymmetry of the $\gamma($ or $Z$ ) with unpolarized or longitudinallypolarized beams, which is even under naive time reversal, hence sensitive only to the 


\section{imaginary part of the vertex.}

In $\gamma Z$ production a $\mathrm{CP}$-violating contribution can arise if anomalous $\mathrm{CP}$-violating $\gamma \gamma Z$ and $\gamma Z Z$ couplings are present. The contributions of the interferences of these anomalous couplings with the SM contribution give rise to a polar-angle forward-backward asymmetry with longitudinally-polarized beams [171]. New combinations of polar and azimuthal asymmetries occur in the presence of transversely-polarized beams [172,173].

Indeed, longitudinal beam polarization may play an important role in improving the sensitivity to absorptive (or real) parts of CP-violating anomalous couplings or form factors, which are measurable even with unpolarized beams through the forward-backward asymmetry. However, transverse polarization enables in addition measurements of dispersive (or imaginary) parts of certain combinations of form factors (or coupling constants) which are inaccessible with longitudinally-polarized beams [173].

The role of transverse polarization in the context of CP violation has previously been studied in section 3.4.1 for the process $e^{+} e^{-} \rightarrow t \bar{t}$. Basically, since transverse beam polarization provides an additional reference axis in addition to the $e^{+} e^{-}$beam direction, there is the possibility of studying the azimuthal distribution of a single final-state particle instead of its polarization via the analysis of its decay distribution.

In the process considered in section 3.4.1, the SM interaction occurs only via $s$-channel $\gamma$ and $Z$ exchanges, and for $m_{e}=0 \mathrm{CP}$-violating effects could only arise from the interference with chirality changing (pseudo)scalar and tensor effective couplings. If, like in the present case of $e^{+} e^{-} \rightarrow \gamma Z, t$ - and $u$-channel exchanges are present, an additional dependence on the scattering (polar) angle $\theta$ exists and those considerations do not apply. Indeed, referring to eq. (3.44), if particle $A$ is self-conjugate the forward-backward asymmetry corresponding to $\theta \rightarrow \pi-\theta$ is CP-odd also in the absence of transverse polarization $[171,174,175]$. Such forward-backward asymmetry would be even under 'naive' time reversal $T$, namely, the reversal of particle spins and momenta but not the exchange of final with initial state. It can be shown that the CPT theorem implies this asymmetry to be proportional to an absorptive part of the interfering amplitudes, hence to the imaginary parts of the new-physics couplings (see, e.g. [172,176]).

However, if there is transverse polarization, a T-odd but CP-even azimuthal asymmetry can be combined with the T-even but CP-odd forward-backward asymmetry to give an asymmetry which is both CP-odd and T-odd. In this case, the CPT theorem would imply that such an asymmetry can measure the real parts of the new-physics couplings that, as stated above, could not be measured without transverse polarization.

Turning now to $e^{+} e^{-} \rightarrow \gamma Z$, where both produced particles are self-conjugate, at the tree level CP violation effects can arise if anomalous CP-violating $\gamma \gamma Z$ and $\gamma Z Z$ couplings are present and interfere with the SM amplitudes [172]".

The parametrization of the anomalous vertex can be done in the most general form consistent with Lorentz invariance, photon gauge invariance and chirality conservation, includes contact interactions as well as triple gauge boson couplings [177], and can be written for $\gamma \gamma Z$ and $\gamma Z Z$ interactions as:

$$
\mathcal{L}=e \frac{\lambda_{1}}{2 m_{Z}^{2}} F_{\mu \nu}\left(\partial^{\mu} Z^{\lambda} \partial_{\lambda} Z^{\nu}-\partial^{\nu} Z^{\lambda} \partial_{\lambda} Z^{\mu}\right)+\frac{e}{16 c_{W} s_{W}} \frac{\lambda_{2}}{m_{Z}^{2}} F_{\mu \nu} F^{\nu \lambda}\left(\partial^{\mu} Z_{\lambda}+\partial_{\lambda} Z^{\mu}\right)
$$

where $c_{W}=\cos \theta_{W}, s_{W}=\sin \theta_{W}$ and $\theta_{W}$ is the weak mixing angle. Notice that $\lambda_{1}$ and $\lambda_{2}$ do not arise in the SM even at loop level, therefore their non-vanishing represents an

\footnotetext{
"For the analysis of the general set of form factors see [173].
} 
unambiguous signal of new physics. Only indirect limits are available from loop-induced weak and electric fermion dipole moments, viz. $\left|\lambda_{2}\right|<10^{-3}$ and similarly for $\lambda_{1}[79,171]$. The interest here, however, is in model-independent direct limits on these constants.

The photon angular distribution contains interference terms dependent on $\operatorname{Re} \lambda_{2}, \operatorname{Im} \lambda_{1}$, $\operatorname{Im} \lambda_{2}$ and proportional to the product of electron and positron degrees of transverse polarization (parallel transverse polarization directions of $e^{+}$and $e^{-}$are assumed). In particular, their dependence as $\cos \theta \cos 2 \phi$ and $\cos \theta \sin 2 \phi$, with $\phi$ the azimuthal angle, are of interest for the determinations of the $\mathrm{CP}$-violating couplings.

Define the following CP-odd asymmetries, that combine a forward-backward asymmetry with an appropriate asymmetry in $\phi$, so as to isolate the anomalous couplings:

$$
\begin{aligned}
& \mathrm{A}_{1}=\frac{1}{\sigma_{0}} \sum_{n=0}^{3}(-1)^{n} \int_{\pi n / 2}^{\pi(n+1) / 2} d \phi\left[\int_{0}^{\cos \theta_{0}}-\int_{-\cos \theta_{0}}^{0}\right] d \cos \theta \frac{d \sigma}{d \Omega}, \\
& \mathrm{A}_{2}=\frac{1}{\sigma_{0}} \sum_{n=0}^{3}(-1)^{n} \int_{\pi(2 n-1) / 4}^{\pi(2 n+1) / 4} d \phi\left[\int_{0}^{\cos \theta_{0}}-\int_{-\cos \theta_{0}}^{0}\right] d \cos \theta \frac{d \sigma}{d \Omega}, \\
& \mathrm{A}_{3}=\frac{2}{\sigma_{0}}\left[\int_{0}^{\cos \theta_{0}}-\int_{-\cos \theta_{0}}^{0}\right] d \cos \theta\left[\int_{0}^{\pi / 4}+\int_{3 \pi / 4}^{5 \pi / 4}+\int_{7 \pi / 4}^{2 \pi}\right] d \phi \frac{d \sigma}{d \Omega},
\end{aligned}
$$

where $\sigma_{0} \equiv \sigma_{0}\left(\theta_{0}\right)$ is the total cross section of the process, integrated with a cut-off $\theta_{0}$ in the polar angle, $\theta_{0}<\theta \leq \pi-\theta_{0}$.

Explicitly, with $P_{e^{-}}^{\mathrm{T}}$ and $P_{e^{+}}^{\mathrm{T}}$ the degrees of transverse polarization, one finds [172]:

$$
\begin{aligned}
& \mathrm{A}_{1}\left(\theta_{0}\right)=-\mathcal{B}^{\prime} g_{A} P_{e^{-}}^{\mathrm{T}} P_{e^{+}}^{\mathrm{T}} \operatorname{Re} \lambda_{2}, \\
& \mathrm{~A}_{2}\left(\theta_{0}\right)=\mathcal{B}^{\prime} P_{e^{-}}^{\mathrm{T}} P_{e^{+}}^{\mathrm{T}}\left[\left(g_{V}^{2}-g_{A}^{2}\right) \operatorname{Im} \lambda_{1}-g_{V} \operatorname{Im} \lambda_{2}\right], \\
& \mathrm{A}_{3}\left(\theta_{0}\right)=\mathcal{B}^{\prime}\left[\frac{\pi}{2}\left[\left(g_{V}^{2}+g_{A}^{2}\right) \operatorname{Im} \lambda_{1}-g_{V} \operatorname{Im} \lambda_{2}\right]+P_{e^{-}}^{\mathrm{T}} P_{e^{+}}^{\mathrm{T}}\left[\left(g_{V}^{2}-g_{A}^{2}\right) \operatorname{Im} \lambda_{1}-g_{V} \operatorname{Im} \lambda_{2}\right]\right] .
\end{aligned}
$$

where

$$
\mathcal{B}^{\prime}=\frac{\alpha^{2}}{16 s_{W}^{2} m_{W}^{2}}\left(1-\frac{m_{Z}^{2}}{s}\right)^{2} \frac{\cos ^{2} \theta_{0}}{\sigma_{0}\left(\theta_{0}\right)} .
$$

It is seen that $A_{1}\left(\theta_{0}\right)$ is proportional to $\operatorname{Re} \lambda_{2}$, while the other two asymmetries depend on both $\operatorname{Im} \lambda_{1}$ and $\operatorname{Im} \lambda_{2}$. These two couplings, therefore, can be constrained independently from the simultaneous measurement of $\mathrm{A}_{2}$ and $\mathrm{A}_{3}$. Notice that the products of electron and positron polarizations appear in the expression of the asymmetries, therefore both $P_{e^{-}}^{\mathrm{T}}$ and $P_{e^{+}}^{\mathrm{T}}$ must be non-zero to observe these CP-violating couplings.

The $90 \%$ C.L. limit that can be obtained with a linear collider with $\sqrt{s}=500 \mathrm{GeV}$, $\mathcal{L}_{\text {int }}=500 \mathrm{fb}^{-1},\left(P_{e^{-}}^{\mathrm{T}}, P_{e^{+}}^{\mathrm{T}}\right)=(80 \%, 60 \%)$, making use of the asymmetries $A_{i}$, has been calculated in [172]. The limiting value $\lambda^{\lim }$ (i.e. the respective real or imaginary part of the coupling) is related to the value $A$ of the asymmetry for unit value of the coupling constant by $\lambda^{\lim }=1.64 /\left(A \sqrt{N_{S M}}\right)$, where $N_{S M}$ is the number of SM events.

$A_{1}$ depends on $\operatorname{Re} \lambda_{2}$ alone, and can therefore place an independent limit on $\operatorname{Re} \lambda_{2}$. It should be emphasized that information on $\operatorname{Re} \lambda_{2}$ cannot be obtained without transverse polarization. The limits are summarized in table 3.12. In the third and fourth columns of this table, the constraints are obtained by assuming $\operatorname{Im} \lambda_{1}$ and $\operatorname{Im} \lambda_{2}$ non-zero, one at a time. In the last column, both $\operatorname{Im} \lambda_{1}$ and $\operatorname{Im} \lambda_{2}$ have been taken simultaneously non-zero in $A_{2}$ and $A_{3}$, which determines an allowed region for those two couplings. 


\begin{tabular}{l|c|c|c|c}
\hline Coupling & \multicolumn{3}{|c|}{ Individual limit from } & Simultaneous limits \\
& $A_{1}$ & $A_{2}$ & $A_{3}$ & \\
\hline $\operatorname{Re} \lambda_{2}$ & $6.2 \times 10^{-3}$ & & & \\
$\operatorname{Im} \lambda_{1}$ & & $6.2 \times 10^{-3}$ & $3.8 \times 10^{-3}$ & $7.1 \times 10^{-3}$ \\
$\operatorname{Im} \lambda_{2}$ & & $9.1 \times 10^{-2}$ & $3.0 \times 10^{-2}$ & $6.7 \times 10^{-2}$ \\
\hline
\end{tabular}

Table 3.12: $90 \%$ CL limits on the couplings from asymmetries $A_{i}$ for a cut-off angle of $\theta_{0}=26^{\circ}$, which optimizes the sensitivity, $\sqrt{s}=500 \mathrm{GeV}$, and $\mathcal{L}_{\text {int }}=500 \mathrm{fb}^{-1}$. Assumed transverse polarizations are $\left(P_{e^{-}}^{\mathrm{T}}, P_{e^{+}}^{\mathrm{T}}\right)=(80 \%, 60 \%)[172]$.

Quantitative examples: Only with both beams transversely polarized is it possible to put separate limits on the real as well as the imaginary part of possible CP-violating anomalous $\gamma \gamma Z$ and $\gamma Z Z$ couplings. The sensitivity is rather high, especially on $\operatorname{Re} \lambda_{2}$, on which a direct reach of about $6.2 \times 10^{-3}$ can be achieved. 


\section{Chapter 4}

\section{Summary of the physics case}

In the previous chapters the benefits from having the positron beams polarized at the linear collider, as well as the electron beam, have been discussed. The physics case for this option has been illustrated by explicitly analyzing reference reactions in different physics scenarios. The results show that positron polarization, combined with the clean experimental environment provided by a linear collider, would allow one to improve dramatically the search potential for new particles and the disentanglement of their dynamics. This would represent a crucial step towards the understanding of the nature of fundamental interactions. Concurrently, the Standard Model and its parameters could be scrutinized and determined with unparallelled precision. The availability of positron polarization would allow significant progress also in this respect.

In direct searches, the physics potential of the ILC is strongly improved if one can exploit simultaneously the independent polarizations of both beams, particularly in the following regards, cf. also table 4.1 .

- The chiral structures of interactions in various processes can be identified independently and unambiguously. This provides the possibility of determining the quantum numbers of the interacting particles and testing stringently model assumptions. Several of these tests are not possible with polarized electrons alone.

- The larger number of available observables is crucial for disentangling the new physics parameters in a largely model independent approach.

- Transverse polarization of both beams enables the construction of new CP-odd observables using products of particle momenta, and further enlarges the number of observables available to constrain the new physics parameters.

- The enhanced rates with suitable polarizations of the two beams would allow for better accuracy in determining cross sections and asymmetries. This increase of the signal event rate may even be indispensable, in some cases, for the observation of marginal signals of new physics.

- A more efficient control of background processes can be obtained. The higher signalto-background ratio may be crucial for finding manifestations of particles related to new physics and determining their properties. Important examples are the searches for signatures of massive gravitons, whose existence is foreseen by models with extra dimensions, and of supersymmetric particles. 
In indirect searches for new physics, the clear advantages of having both beams polarized simultaneously include the following.

- The enhancement of cross sections, and correspondingly of the rates, by effective use of the polarizations, leads to a reduction in the statistical uncertainties.

- Significant increases in sensitivity to new physics at high energy scales can be achieved, with the possibility to elucidate the associated interactions.

- The increase in the sensitivity to non-standard couplings due to the synergy of high energy, high luminosity and especially of different possible initial polarization configurations will allow one to disentangle different kinds of interactions.

- The left-right asymmetry, which can be crucial for distinguishing different models, is often limited by systematic uncertainties. These can be reduced significantly when both beams are polarized.

- There is an increase in the sensitivity to new interactions which are not of the currentcurrent type, such as those mediated by gravitons or (pseudo)scalar exchanges.

Transversely polarization of both beams opens up new possibilities:

- This offers new observables to detect non-standard interactions, including possible new sources of $\mathrm{CP}$-violation.

- It enhances the sensitivity to graviton interactions and enables one to draw a distinction between different scenarios of extra spatial dimensions, even far below the resonance production threshold.

- It provides access to specific triple-gauge couplings which cannot be extracted with only longitudinally polarized beams.

The precision tests of the Standard Model are unprecedented. In particular, the use of simultaneously polarized beams improves significantly the precision attainable in the following measurements.

- It improves the separation between the annihilation and scattering channels in $W^{+} W^{-}$ production, as required for an optimal determination of triple-gauge-boson couplings.

- High-luminosity GigaZ running at the $Z$ boson resonance or at the $W^{+} W^{-}$threshold with positron polarization allows for an improvement in the accuracy of the determination of $\sin ^{2} \theta_{W}$ by an order of magnitude, through studies of the left-right asymmetry. This will have far-reaching implications for consistency tests of the electroweak theory, in particular in the Higgs sector.

The qualitative and quantitative consequences of having both beams longitudinally polarized in comparison with the $e^{-}$polarization only for the reference reactions analysed in the previous chapters are also listed in table 4.1. These examples by no means exhaust the whole phenomenology of a polarized positron beam. The quantitative improvement factors are relative to the case with polarized electrons only. In most cases $\left|P_{e^{-}}\right|=80 \%$ and 
$\left|P_{e^{+}}\right|=60 \%$ are used as conservative possibilities, but in some examples also $\left|P_{e^{-}}\right|=90 \%$ and $\left|P_{e^{+}}\right|=80 \%$ or $40 \%$ have been considered.

It is apparent from the table that in some specific cases of non-standard couplings factors of about an order of magnitude in the Figur-of-Merit, the $S / \sqrt{B}$ ratio, could be gained when employing two polarized beams, compared with the case of polarized electrons only. Since in some new physics models only very small rates are expected, such a large gain in the Figur-of-Merit may be crucial to detect the signals. In some examples, such as testing the quantum numbers and properties of supersymmetric particles, simultaneously polarized beams are definitely needed.

Indirect searches are a very important tool to find signatures of new interactions characterized by higher mass scales far beyond the kinematical reach of present, future and next-generation collider experiments. Such new mass scales can be accessed by looking for deviations of experimental observables from the Standard Model predictions, and in principle the results depend sensitively on the particular model adopted in order to analyse the data. Since the discovery reach scales with the luminosity, it is often enhanced by about $20 \%$ with both beams polarized compared to the case with only polarized electrons. Furthermore, the positron polarization option allows more observables to be measured, reducing to a large extent the model dependence. The use of polarized positrons also improves substantially the precision with which the left-right asymmetry observables can be measured.

Specific advantages of transverse beam polarization are listed in table 4.1. For example, in indirect searches for massive gravitons, one could even distinguish between different models of the extra dimensions if both beams are transversely polarized. Furthermore, transverse polarization turns out to be particularly promising in the context of searches of new CP-violating interactions, for example by exploiting azimuthal asymmetries that may allow the detection of even marginal manifestations of new sources of $\mathrm{CP}$ violation.

The physics studies are still ongoing, and the listed examples should be understood solely as a contemporary status report*. Nevertheless, these examples already show that positron polarization has excellent possibilities for enrichening considerably the physics output from the linear collider and that the full potential of the ILC could be realized only with a polarized positron beam as well.

${ }^{*}$ For further information, see http://www.ippp.dur.ac.uk/ gudrid/power/ . 


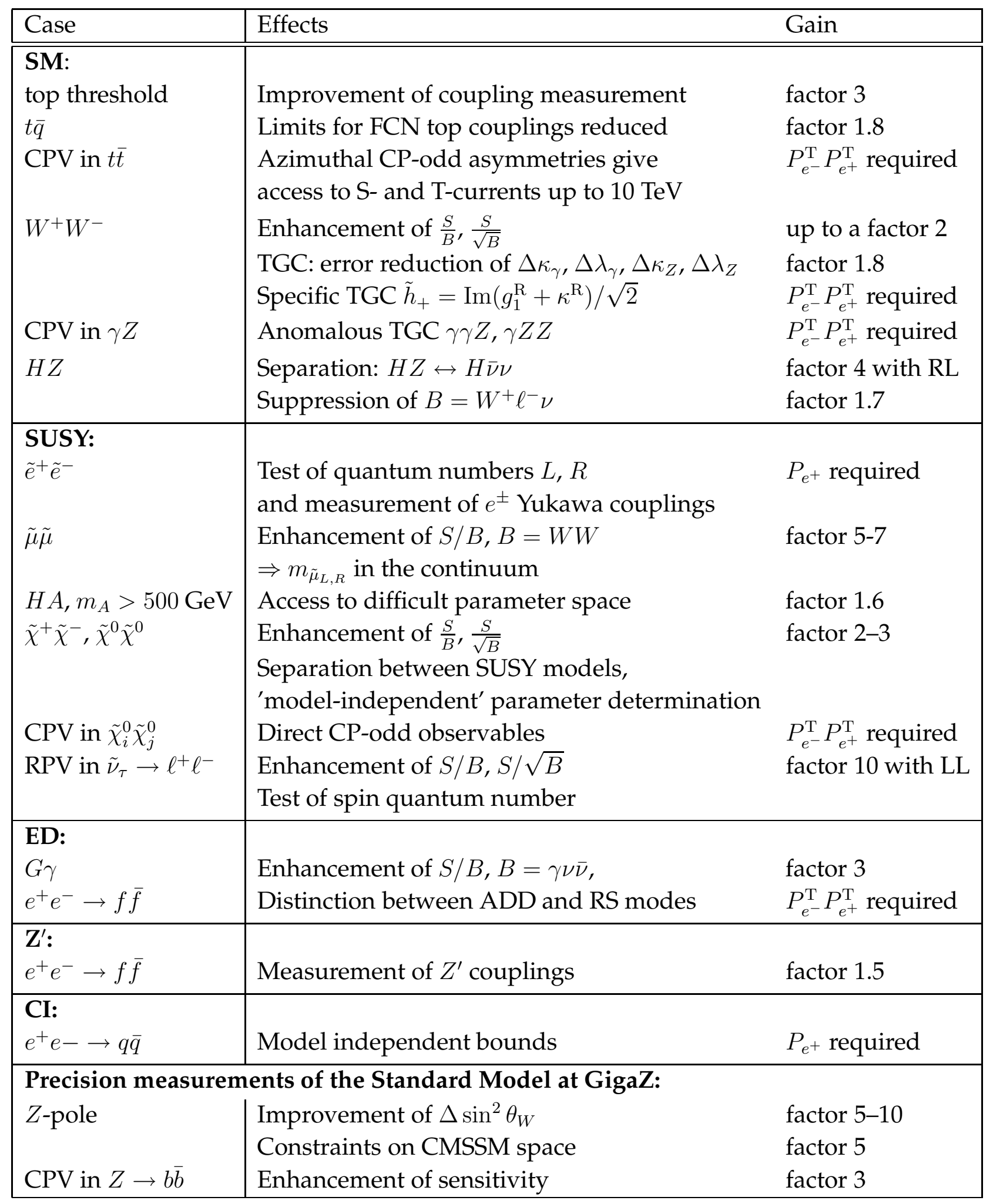

Table 4.1: Some of the physics examples given in this report. The case of having both beams polarized is compared with the case of using only polarized electrons; in many cases $\left(\left|P_{e^{-}}\right|,\left|P_{e^{+}}\right|\right)=(80 \%, 60 \%)$ is compared to $\left(\left|P_{e^{-}}\right|,\left|P_{e^{+}}\right|\right)=(80 \%, 0 \%)$, cf. see corresponding chapter; B (S) denotes background (signal); CPV (RPV) means CP (R-parity) violation. 


\section{Chapter 5}

\section{Machine issues}

Polarized positrons are produced via pair production from circularly-polarized photons. The main issues associated with the development of a polarized-positron source for a linear collider are: the generation of polarized photons, the efficient capture of the positrons, and dealing with the high heat loads (and accompanying radiation) due to the required flux. This chapter discusses the photon-based schemes for polarized-positron production being considered for the ILC. Status updates of work towards the realization of viable polarized-positron sources are also given. A report of work on polarized-electron sources is included, followed by a discussion of the spin manipulation systems required in the ILC. The chapter continues with a section about measuring the polarization with conventional polarimetry. Emphasis is put on Compton polarimetry and designs for polarimetry up- as well as downstream of the IP are discussed. However, an overview of other methods such as Møller polarimetry is also given and future design work is outlined. The chapter concludes with a section about measuring the polarization from physics processes in $W$ and $W$-pair production and from collider data via application of the Blondel scheme.

\subsection{General remarks}

In this section, a short introduction to polarized positron production is given along with brief descriptions of the possible sources of polarization loss. A description of the polarized electron source for the ILC is included for completeness.

The current parameter specifications for the ILC list polarized electrons with $P_{e^{-}} \geq$ $80 \%$ for the baseline machine whereas positron polarization (with $P_{e^{+}} \geq 50 \%$ ) is suggested as an option for a later upgrade [3]. If adequately planned in the baseline, the upgrade to polarized positrons is relatively straight forward and not prohibitively expensive in terms of either cost or downtime required to implement the upgrade. The two main methods for positron production under consideration for the ILC are a photonbased source and a 'conventional' source. The photon-based source uses multi-MeV photons and relatively thin targets (less than a radiation length thick) to produce positrons. If the photons are circularly polarized, the positrons (and electrons) are spin polarized. This positron polarization can be preserved in the subsequent capture, acceleration, damping, and transport to the collision point(s). The conventional source uses a multi-GeV electron 
drive beam in conjunction with thick, high- $Z$ targets to produce positrons from the resultant electromagnetic cascade in the target. The positrons produced by this method cannot be polarized. These two schemes ultimately present very similar engineering challenges while at the same time having distinct attributes and drawbacks. It is emphasized, however, that only the photon-based schemes offer the promise of positron polarization [178].

In addition to developing a source of polarized positrons (and electrons) for a high energy linear collider, it is important to ensure that no significant polarization is lost during the transport of the positron and electron beams from the source to the interaction region. Transport elements downstream of the sources which can contribute to a loss of polarization include the initial acceleration structures, transport lines to the damping rings, the damping rings, the main linacs, and the high energy beam delivery systems. As discussed below, the largest depolarization effect is expected to result from the collision of the two beams at the interaction point(s). This effect is expected to decreases the polarization by about $0.25 \%$, see section 5.5 .

\subsubsection{Polarized-positron source}

Circularly-polarized photons are required for the generation of longitudinally-polarized positrons via $e^{ \pm}$pair production in a thin target. The photons are in the energy range of a few $\mathrm{MeV}$ up to about $100 \mathrm{MeV}$. Because the target is typically a fraction of a radiation length thick, high strength materials, such as titanium alloys, can be considered as opposed to conventional targets, where high-Z, high-density materials are required to minimize the emittance of the produced positrons. The two methods for generating the polarized photons under consideration are:

- a high-energy electron beam ( $\gtrsim 150 \mathrm{GeV}$ ) passing through a short period, helical undulator. This scheme is discussed in greater detail in sections 5.2.1, 5.2.2. The E-166 experiment, which is currently running at SLAC, is a demonstration of this undulator-based polarized positron production scheme. The experiment will be finished in 2005.

- Compton backscattering of laser light off a GeV energy-range electron beam. This is discussed in detail in section 5.2.3 and the concept is being tested in an experiment which is currently running at KEK.

In both schemes a positron polarization of about $\left|P_{e^{+}}\right| \geq 60 \%$ is expected at the ILC.

Both schemes would be applicable for the ILC design [3] and also adaptable for a possible future multi-TeV LC design [6].

\section{Comparison with conventional (unpolarized) positron sources}

Apart from the obvious advantage of generating polarized positron beams, photon drive beam based positron sources have various advantages and disadvantages as compared with conventional sources using electron drive beams. At this time both the conventional and undulator-based positron source designs are equally mature and can meet the ILC requirements. The big advantage of the conventional source is that is completely decoupled from the rest of the ILC whereas the undulator-based positron source needs a working ILC main linac for operation. This leads to some advantages in commissioning 
and uptime. With careful design and an additional simple keep-alive positron source, these advantages can be mitigated, though not completely eliminated.

The photon-based positron source has three advantages over the conventional source [179].

- First the fact that the target is thinner allows for the use of lower- $Z$ materials such as Ti-alloys that are stronger than the W-Re alloys used in the conventional sources.

- Secondly the photon energy can be chosen to be lower than the neutron photoproduction cross-section and the activation of the target material may be much reduced.

- Thirdly the emittance of the produced positrons is less because the target is thin. This will have advantages for the design of the positron damping ring(s) and maybe reduce the cost advantages of the conventional source.

Cost and availability issues associated with an undulator-based positron source are discussed in [180]. In this study, a comparison is made between an undulator-based system and a conventional (unpolarized) system. Whereas the detailed assumptions and quantitative results of [180] are open to discussion, the general conclusions are reasonable: the cost difference between an undulator source and an upgrade from a conventional source is small $(\sim 1 \%)$ in comparison to the overall project cost; availability and schedule are impacted due to the requirement of high energy electrons for undulatorbased positron production. These issues need to be addressed in future design studies.

\subsubsection{Polarized electron source}

The polarized electron source consists of a polarized high-power laser beam and a highvoltage dc gun with a semiconductor photocathode. This design was developed and successfully used at the SLC and is currently in use at CEBAF. The SLC system can be adopted directly for the ILC using a laser system with the appropriate time structure. While there is discussion about the benefits of a low-emittance radio frequency (RF) gun for polarized-electron production, no $R \& D$ is ongoing or planned for such a gun. Recent work in the area of polarized electron source design for the ILC is presented in section 5.3 and it is expected to achieve a degree of electron polarization of about $\left|P_{e^{-}}\right| \leq 90 \%$ at the ILC.

\subsubsection{Polarization preservation}

\section{Low-energy beam capture and acceleration:}

The transverse and longitudinal phase space of the positrons coming out of the target is matched into the 6-D acceptance of the downstream system by a longitudinally varying solenoidal field. The positrons are then accelerated up to the damping-ring energy of 5 $\mathrm{GeV}$. Axial solenoid fields are used for the transverse capture optics at the low energies up to about $250 \mathrm{MeV}$. Thereafter, magnetic quadrupoles are used for focusing. The longitudinal polarization is preserved while the transverse component is washed out due to spin precession in the solenoidal magnetic fields used for the initial capture. Because of the high incident beam power on the target and constraints in the mechanical design of positron targets, multiple target stations running in parallel may be required for the ILC. 
The layouts of these target stations can be configured so that the polarization remains longitudinal after bunches from the different targets are combined into the ILC beam. Space charge effects are not expected to be a source of depolarization. Because of the lower energy, space-charge forces are significantly larger in the case of the polarized-electron source but even in this case no spin depolarization has been observed.

\section{Damping rings:}

Only polarization parallel or anti-parallel to the guide fields of the damping ring is preserved. Thus the incoming longitudinal polarization must be rotated into the vertical prior to injection into the rings. This is done in the transport line leading from the end of the $5 \mathrm{GeV}$ linac to the damping ring, utilizing an appropriate combination of dipole and solenoid fields. The vertical spins can be rotated back to the longitudinal direction with an analogous system in the damping ring extraction line leading to the main linac. These spin manipulation systems are discussed in further detail in section 5.4.

It is important to avoid spin-orbit coupling resonance depolarizing effects by operating off the resonant energies. Depolarization due to the stochastic nature of photon emission can be estimated by comparing the time that a beam spends in the damping ring to the time constant for depolarization. This ratio is typically very small and hence depolarization in the rings is expected to be vanishingly small. Nevertheless it is important to revisit these issues as the ring designs become available and a detailed simulation of depolarization effects should be made. This is best carried out using a Monte-Carlo simulation of the spin-orbit motion [181-183].

\section{Main linac:}

In a linac, the electric field and the particle velocity are essentially parallel. Then, according to the Thomas-Bargmann-Michel-Telegdi (T-BMT) equation the electric field will cause negligible spin precession. The effects of the transverse field from the RF couplers and quadrupoles are also negligible. There should be no loss of polarization in the main linac.

\section{Beam delivery system:}

After acceleration in the main linacs, the $e^{ \pm}$beams are brought to collision by the beam delivery systems. These beam lines contain bend magnets. According to the T-BMT equation, a beam deflection of $\delta \theta_{b}$ in a transverse magnetic field causes a spin rotation of $a \gamma \delta \theta_{b} \approx E(\mathrm{GeV}) / 0.441(\mathrm{GeV}) \delta \theta_{b}$. Depolarization associated with synchrotron radiation in the transport system bends is expected to be negligible. Care is needed to ensure that the polarization is longitudinal (or perhaps transverse) as needed at the interaction point. The spin rotator systems between the damping rings and the main linacs permit the setting of arbitrary polarization vector orientations at the IP.

\section{Beam-beam interactions:}

Loss of polarization can also occur as the electron and positron bunches collide. The two mechanisms responsible for this are spin rotation, according to the T-BMT equation, of the 
spins in one bunch due to the electric and magnetic fields in the oncoming bunch; and spin flip due to synchrotron radiation (the Sokolov-Ternov effect). The combined effect of these two mechanisms has been studied analytically [184] as well as numerically [185, 186]. At $500 \mathrm{GeV}$ center-of-mass energy for the nominal ILC parameters [3], the expected overall loss of polarization [185] of either beam is expected to be $\Delta P / P \simeq 1 \%$. However, the effective loss up to the point of interaction (luminosity-weighted) is only $\simeq 0.25 \%$ : see section 5.5 for more detail. Updates of these calculations using actual design parameters are needed as the machine parameters become available.

\subsection{Positron polarization}

Polarized positrons are produced via pair production from circularly-polarized photons. The concepts under consideration for polarized-positron sources differ only in the manner in which the circularly-polarized photons are produced: either through the use of a helical undulator or from Compton backscattering. The issues associated with target engineering, optimization of the capture yield and positron polarization are identical for both types of schemes.

This section begins with a discussion of undulator-based production of polarized positrons. The basic concept is presented and followed by a description of the SLAC E166 demonstration experiment. A summary of work at Daresbury on the development of undulator prototypes concludes the discussion of the undulator-based sources. The design of a laser-Compton based polarized positron source is described in 5.2.3. A discussion of a demonstration experiment which has been developed at the KEK ATF is included.

\subsubsection{Undulator-based polarized positron source}

A polarized positron source based on the radiation from an undulator was first proposed by Balakin and Mikhailichenko [187] in 1979 in the framework of the VLEPP project, several prototypes for short period helical super-conducting undulators have been proposed [188]. This method requires a multi-hundred $\mathrm{GeV}$ electron beam and in order to save on ILC cost, it is proposed to use the electron main linac to provide this beam. Fig. 5.1 shows the layout of the polarized positron source as developed for the USLCTOS cold reference design [180]. The electron beam is extracted from the electron main linac at an energy of about $150 \mathrm{GeV}$ and transported through $\sim 200 \mathrm{~m}$ of the helical undulator. The electrons are then injected back into the linac and are used for collisions. The period of the undulator is nominally $1 \mathrm{~cm}$ and the magnetic field on axis has a strength of about $\mathrm{B}=1.1 \mathrm{~T}$. Photons generated in the undulator have an energy of the order of $10 \mathrm{MeV}$ and are circularly polarized. These photons are incident on a thin target (about 0.4 radiation lengths). Within the target, the photons convert to electron-positron pairs. The helicity of the initial state is conserved in the pair creation process. As described in detail in [189], the spin orientation of the created positron is correlated to its energy relative to that of the initial, polarized photon. The polarization of the beam of positrons that is collected from the target falls in the range of $40 \%-70 \%$, and depends on the details of the initial photon polarization spectrum, target material and thickness, and capture phase space. This scheme provides also a high intensity of polarized positrons. 
The TESLA proposal has the undulator placed at the end of the linac with consequentially higher energy electrons and photons [190]. While the details of the two designs vary due to the different available electron energy (150 GeV in the case of the USLCTOS design and $250 \mathrm{GeV}$ in the case of TESLA design), see fig. 5.1, either scenario (TESLA or USLCTOS) can be used to produce a beam of polarized positrons for the ILC. Operational aspects of the two different scenarios are somewhat different. The USLCTOS design is based largely on the TESLA design with the difference that the undulator is placed along the electron linac rather than at the end. This is done so that the electron drive beam energy does not vary if the collision energy is changed. This design choice requires that a linac bypass must be built which does not adversely dilute the electron emittance. A principle concern in the design is the effect of the limited energy passband of the bypass with regard to machine protection in the event of a sudden loss of electron energy. Design effort to mitigate this problem is ongoing.

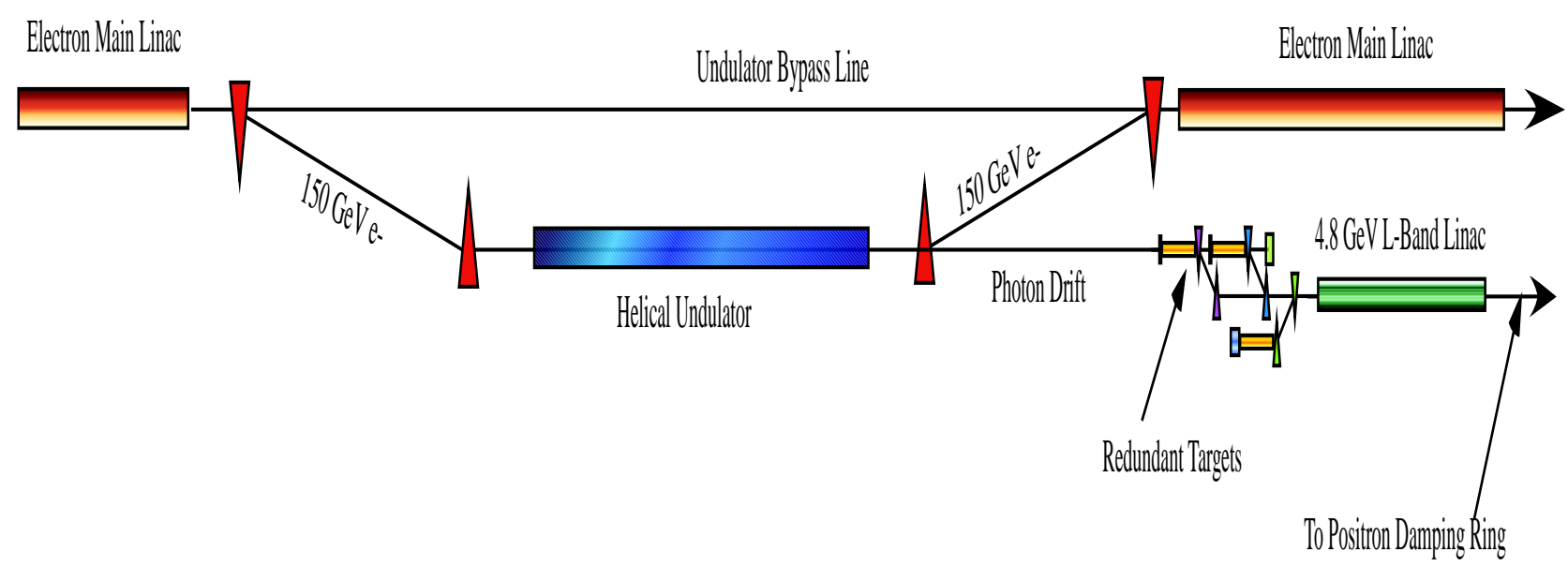

Figure 5.1: A possible layout of the polarized positron source at the ILC. The electrons could be extracted form the main linac with an energy of $150 \mathrm{GeV}$, as shown in the figure [180]. The undulator could also be placed at the end of the linac, where the electrons have an energy of $250 \mathrm{GeV}$ [190].

The SLC positron source is a good starting point for extrapolation to a positron source for the ILC. However, the required ILC positron source flux is a factor of about 60 greater than that in the SLC positron target system. The power of the incident drive beam and the energy deposited in the target station and downstream systems is a factor of about 10 greater than in the SLC design. In addition, the pulse structure of the ILC beam is significantly different from that at the SLC. The discrepancy between the factor of 60 in flux and the factor of 10 in dissipated power is due to the expected increase in the acceptance of the positron capture systems with respect to the SLC design. Both the TESLA Design Report and USLCTOS have descriptions of plausible system designs. The design effort for the ILC positron source is aimed at realizing the increase in positron capture efficiency and handling the increase in power. This engineering effort spans the entire range of component parameter space in the quest to maximize the positron polarization while minimizing the incident beam power. It should be noted that the design issues associated with both the unpolarized conventional and the photon-based systems are essentially 
the same for the systems downstream of the target: the matching optics, RF capture sections, and damping ring acceptance. Even the power absorption in the conventional and photon-based target systems is very similar. The work to develop a viable positron source includes the engineering and testing of high strength target materials, development and testing of magnetic capture optics, and prototyping of the RF structures used for the initial acceleration and the target vault design and associated remoted handling systems.

Some effort is still required to meet the engineering challenges, whether or not positron polarization is chosen for the ILC baseline.

\subsubsection{Demonstration experiment E-166}

A demonstration experiment for undulator-based polarized-positron production at the ILC has been approved at SLAC [191]. This experiment, E-166, is scheduled to run in 2005 in the SLAC Final Focus Test Beam (FFTB) area. The aim of the experiment is to test the fundamental process of generating circularly-polarized photons in a helical undulator [192] and the production of polarized positrons via pair production in a thin target with these photons. In addition, the parameters of the experiment were chosen so that the photon spectrum would be the same as that for the USLCTOS design and E166 would be a scaled down demonstration of a polarized ILC positron source.

A schematic layout of E166 is shown in fig. 5.2. The FFTB electron beam passes through a 1-m long pulsed helical undulator to generate the circularly polarized photons. Because the FFTB beam can have a maximum beam energy of about $50 \mathrm{GeV}$, instead of the higher energies for the ILC design, the undulator aperture has to be reduced to less than $1 \mathrm{~mm}$ in order to generate the same photon spectrum (approximately $10 \mathrm{MeV}$ cut-off for the first harmonic of the undulator radiation). The photons are converted to positrons (and electrons) in a thin, moveable target. Titanium and tungsten targets, which are both candidates for use in linear colliders, will be tested. The experiment will measure the flux and polarization of the undulator photons, as well as the spectrum and polarization of the positrons produced in the conversion target, and compare the results of measurement with simulations. Thus the proposed experiment directly tests the validity of the simulation programs used for the physics of polarized pair production in finite matter, in particular the effects of multiple scattering on polarization.

Table 5.1 provides a comparison of the E-166 parameters with those of the unpolarized TESLA TDR design and of the polarized design of the USLCTOS cold option. It is seen that E-166 produces photons in the same energy range, uses similar target materials and thicknesses, and produces positrons with the same polarization characteristics as those in the two linear collider designs.

The achievable precision of $5-10 \%$ of the proposed transmission polarimetry is sufficient. Good agreement between the experimental results and the simulations will lead to greater confidence in the proposed designs of polarized-positron sources for the next generation of linear colliders.

This experiment, however, will not address detailed systems issues related to polarizedpositron production at a linear collider, such as capture efficiency, target thermal hydrodynamics, radiation damage in the target, or an undulator prototype suitable for use at the ILC; such issues are well within the scope of R\&D of a linear collider project that chooses to implement a polarized-positron source based on a helical undulator. 


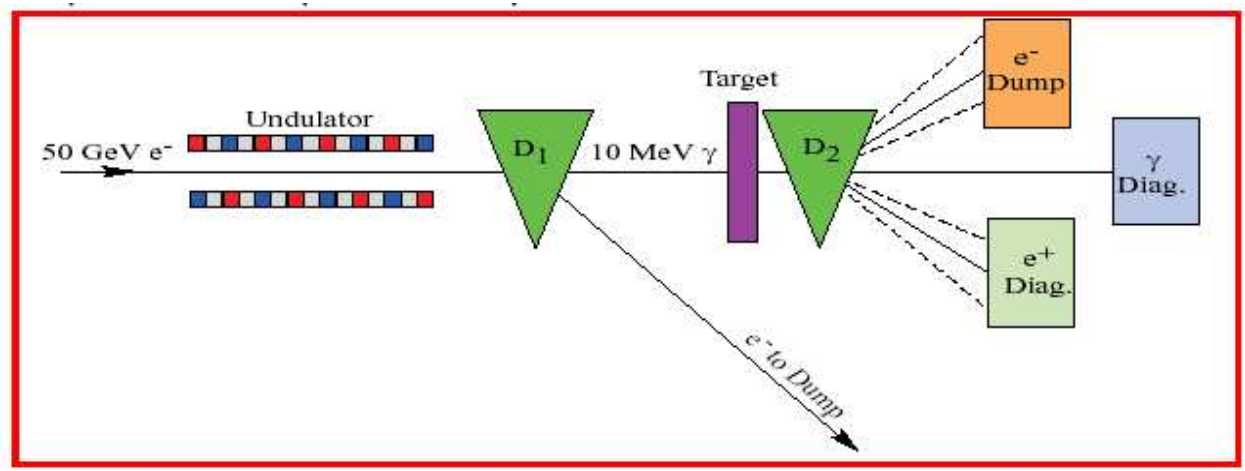

Figure 5.2: Conceptual layout of the experiment to demonstrate the production of polarized positrons in the SLAC FFTB. The $50-\mathrm{GeV} e^{-}$beam passes through an undulator, producing a beam of circularly polarized photons of $\mathrm{MeV}$ energy. The electrons are deflected by the $D_{1}$ magnet. The photons are converted to electrons and positrons in a thin Ti target. The polarizations of the positrons and photons are measured in polarimeters based on Compton scattering of electrons in magnetised iron [191].

\begin{tabular}{|c|c|c|c|}
\hline Parameter & TESLA & USLCTOS & E-166 \\
\hline Beam Energy, $E_{e}[\mathrm{GeV}]$ & $150-250$ & 150 & 50 \\
\hline$N_{e} /$ bunch & $3 \times 10^{10}$ & $2 \times 10^{10}$ & $1 \times 10^{10}$ \\
\hline$N_{\text {bunch }} /$ pulse & 2820 & 2820 & 1 \\
\hline Pulses/s [Hz] & 5 & 5 & 30 \\
\hline Undulator Type & plan./helical & helical & helical \\
\hline Und. Parameter, $K$ & 1 & 1 & 0.17 \\
\hline Und. Period, $\lambda_{u}[\mathrm{~cm}]$ & 1.4 & 1.0 & 0.24 \\
\hline Und. Length, $L[\mathrm{~m}]$ & 135 & 200 & 1 \\
\hline $1^{s t}$ Harmon., $E_{c 10}[\mathrm{MeV}]$ & $9-25$ & 11 & 9.6 \\
\hline Target Material & Ti-alloy & Ti-alloy & Ti-alloy, W \\
\hline Target Thickn. [rad. len.] & 0.4 & 0.4 & $0.25-0.5$ \\
\hline Pos. Pol. [\%] & 0 & 59 & 53 \\
\hline
\end{tabular}

Table 5.1: Positron parameters for the unpolarized TESLA baseline design, polarized USLCTOS design, and the E-166 experiment [191]. 


\subsubsection{Helical undulator development}

Two helical undulator prototypes for the ILC are being developed under the guidance of the ASTeC group at Daresbury Laboratory [193]. The first device uses a bifilar helix of niobium-titanium superconducting wire; the second device is based on neodymiumiron-boron permanent magnet material. Table 5.2 lists the parameters of the magnets. An undulator period of $14 \mathrm{~mm}$ has been selected for the prototypes and fig. 5.3 gives an indication of the magnetic fields required to produce photons of approximately the correct energy. The parameters have been chosen to meet the requirements of the TESLA TDR, and can be scaled to the current ILC design. In table 5.2, 'Length' refers to the prototype and 'Full Module' is the length of an undulator section as envisaged for the ILC. The full ILC undulator consists of a large number of modules to make up the $\sim 200 \mathrm{~m}$ total required length. Studies to date have shown that both the superconducting and permanent magnet technologies will meet the ILC undulator design criteria. To help in deciding which design is better, short 10- to 20-period models have been developed. These models will enable:

- the field quality and strength of each design to be checked.

- assessment of the size and probability of magnet errors.

- the evaluation of the effort required for full-sized module construction.

- an improvement of cost estimates.

\begin{tabular}{|c|c|c|c|c|c|c|c|}
\hline Type & Period & On-Axis Field & Magnet Aperture & Material & Current density & Length & Full Module \\
\hline SC & $14 \mathrm{~mm}$ & $0.85 \mathrm{~T}$ & $4 \mathrm{~mm}$ & NbTi & $1000 \mathrm{~A} / \mathrm{mm}^{2}$ & $30 \mathrm{~cm}$ & $2-5 \mathrm{~m}$ \\
\hline PPM & $14 \mathrm{~mm}$ & $0.83 \mathrm{~T}$ & $4 \mathrm{~mm}$ & NdFeB & $(1.3 \mathrm{~T})$ & $15 \mathrm{~cm}$ & $2-5 \mathrm{~m}$ \\
\hline
\end{tabular}

Table 5.2: Parameters of the prototype of a super-conducting (SC) and permanent-magnet (PPM) helical undulator design parameters [193].

It was decided that both prototype devices would have the same period of $14 \mathrm{~mm}$ to facilitate the comparison. Modelling of the superconducting device indicates that an onaxis field greater than the specified value of $0.85 \mathrm{~T}$ is possible with the inclusion of iron. The NdFeB undulator can also achieve a greater field strength than the $0.83 \mathrm{~T}$ listed in table 5.2. Higher fields permit a reduction in the period. However magnet inhomogeneities, magnetization vector misalignments (in the case of the PPM device), and assembly errors combine to reduce the field quality. The dependence of the field quality on these errors is one of the areas under investigation.

For the superconducting undulator, two wires are wrapped in a double helix around the vacuum vessel [194]. When current is passed through the wires the longitudinal components of the magnetic field cancel leaving only the rotating (helical) field required on axis. A 20 period $(30 \mathrm{~cm})$ long prototype has been made using $\mathrm{NbTi}$ wire. This device is currently being tested.

In the case of the permanent magnet device, each period of the undulator is divided into slices. Each slice comprises a ring of trapezoidally shaped permanent magnet material blocks whose magnetization vectors rotate through $720^{\circ}$ around the ring to produce a 


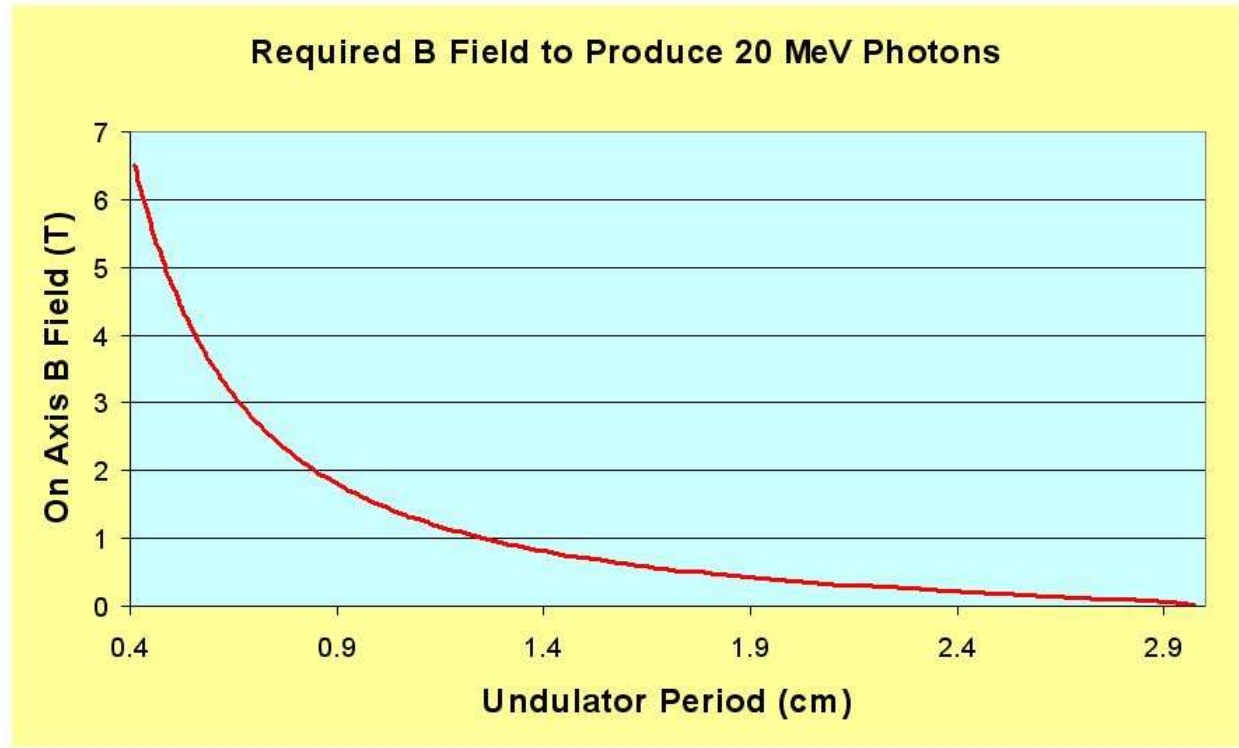

Figure 5.3: On-axis B field versus undulator period to produce circularly-polarized $20 \mathrm{MeV}$ photons with a $250 \mathrm{GeV}$ electron beam [193].
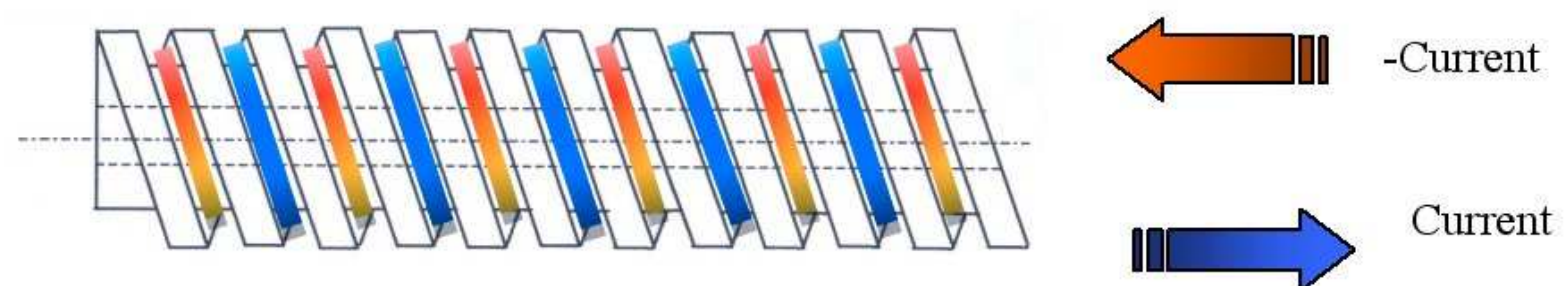

Figure 5.4: Schematic of wires wrapped in a helix around a former showing different current directions [193].

transverse dipole field [195]. Adjacent rings are rotated through $360^{\circ}$ over one undulator period to form a helical field pattern. By changing the number of blocks in a ring and the number of rings in a period, the field strength and quality is altered. An $8 \times 8$ prototype is being developed which uses 8 blocks to form a ring with 8 rings to a period. The device is 10 periods long or about $15 \mathrm{~cm}$, including end pieces. This undulator will be ready for testing in the spring of 2005.

\subsubsection{Laser-Compton based polarized positron source}

Polarized positrons can be also created by a laser-based scheme, where circularly polarized gamma-photons are obtained by backscattering laser light off an electron beam. These circularly polarized gammas are then used to generate longitudinally-polarized positrons via pair production in a thin radiator in the same manner as in the undulatorbased case. One of the advantages of this scheme is that because the required electron beam has a few $\mathrm{GeV}$ and can be generated in a stand-alone linac, the positron source is independent of the electron main linac in contrast to the case with the undulator-based source. The main technical challenge in this scheme is to realize the total laser light intensity needed for an ILC-capable positron source. Three possible ways are under consider- 


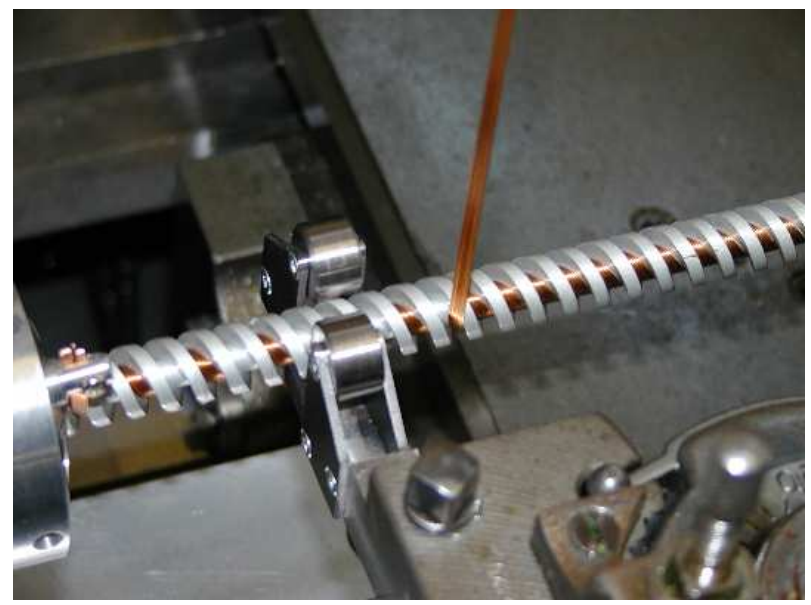

Figure 5.5: Undulator prototype with super-conducting (SC) wires being wound [193].
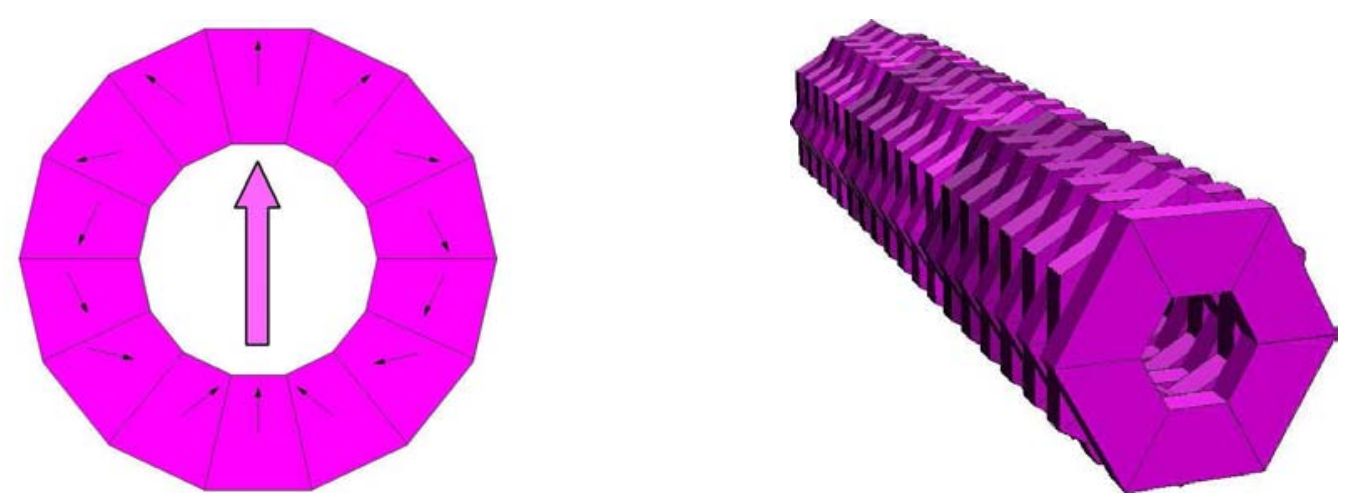

Figure 5.6: Dipole field created by many permanent-magnets (PM) blocks arranged in a ring. Many rings are stacked together and rotated to create the helical field [193]. 
ation to realize this scheme [196].

- The first uses $\mathrm{CO}_{2}$ lasers. Collision of a $5.8 \mathrm{GeV}$ electron beam and $\mathrm{CO}_{2}$ laser light creates photons with a maximum energy of $60 \mathrm{MeV}$, see fig. 5.7, whose energies are suitable for positron production. A multi-bunch pulse $\mathrm{CO}_{2}$ laser needs to be developed for this purpose. The possibility currently considered is to create 100 laser bunches with an inter-bunch spacing of 2.8 nsec. A $5.8 \mathrm{GeV}$ electron beam must then have the same bunch time spacing to get collisions of all bunches. The above design is similar to the design for a warm LC [197].

To meet ILC requirements, about 30 lasers are used to obtain 3000 bunches. Since the ILC operates at $5 \mathrm{~Hz}$, one has $200 \mathrm{msec}$ between pulses. $100 \mathrm{msec}$ are used for the generation of the 3000 bunches and $100 \mathrm{msec}$ are used for damping. Fast kickers are necessary to handle such bunches when one injects (extracts) into (from) a damping ring.

- A second technique employs FELs of $\mathrm{CO}_{2}$ wave length for the light source [198]. By using an FEL, one can produce 3000 bunches of light in one pulse. The electron beam also has 3000 bunches to get collisions with all photon bunches. The bunch spacing of both beams should be identical for that purpose. The bunch spacing can be chosen in a very wide range when one employs FELs as light sources: for example one can choose a bunch time spacing of $2.8 \mathrm{nsec}, 20 \mathrm{nsec}$, or $300 \mathrm{nsec}$.

- Lastly, a new idea requires accumulation of positrons in a ring. Since the ILC operates at $5 \mathrm{~Hz}$, one has $100 \mathrm{msec}$ to accumulate positrons and get the required population. Then the number of positrons created in one collision can be drastically reduced. Thus the collision repetition rate should be very high (more than $\mathrm{MHz}$ ) in order to get the required population. To achieve this, one proposal is to use pulse stacking by inserting a cavity of laser light in an electron ring [199]. Another proposal to realize high-repetition collisions is to use a high-repetition FEL based on ERL (energy recovery linac) technology developed for light sources [200].

Work is still necessary to develop these schemes to the point where they can be considered as viable options for polarized positron production.

\subsubsection{Laser-Compton demonstration experiment}

An experiment is ongoing at the KEK-ATF to make a proof-of-principle demonstration of laser-based schemes, and to develop the polarimetry of short pulses of photons and positrons. In this experiment, a $1.28 \mathrm{GeV}$ electron beam from the ATF and the 2nd harmonic of a YAG laser are used to produce polarized photons with a maximum energy of $56 \mathrm{MeV}$ [201]. The collision point is located in the extraction line of the ATF. At the collision point a specially designed chamber, the Compton chamber, is installed; fig. 5.8. This chamber has screens at the collision point, and also downstream and upstream of the collision point. By using these screens, the positions and angles of the laser and electron beams are adjusted in order to realize accurate head-on collisions. The Compton chamber also has a wire scanner and a knife-edge scanner to measure the spot sizes of the electron and laser beams at the collision point. The polarization of the produced photons is measured by a transmission method. In this method the intensities of transmitted photons 


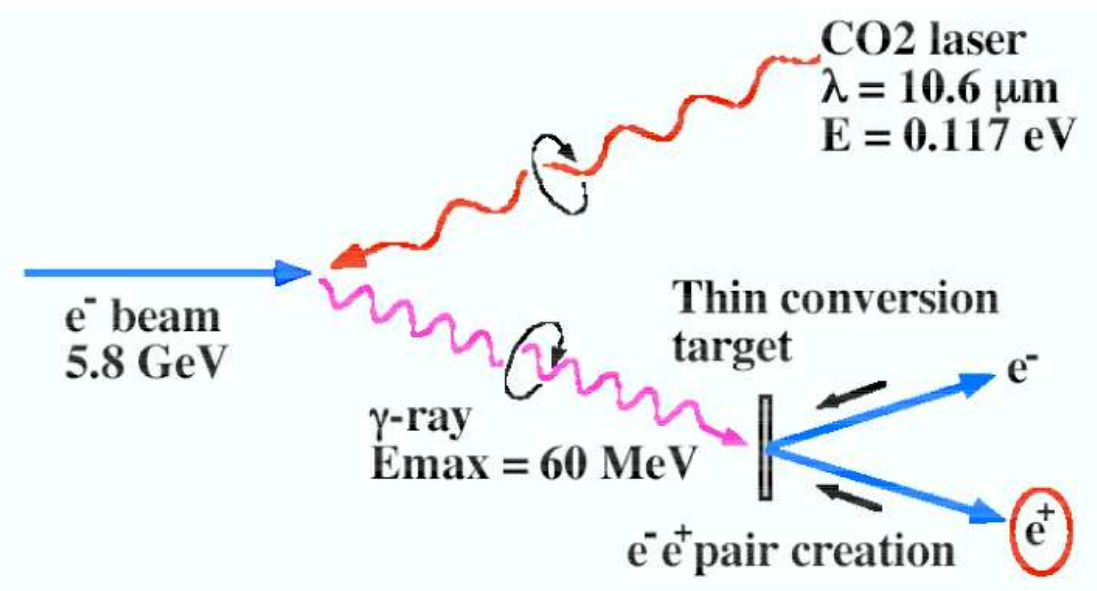

Figure 5.7: Collision of a $5.8 \mathrm{GeV}$ electron beam and $\mathrm{CO}_{2}$ laser light creates circularlypolarized gamma-photons with maximum energy of $60 \mathrm{MeV}$. Polarized positrons are then generated via pair production in a target [197].

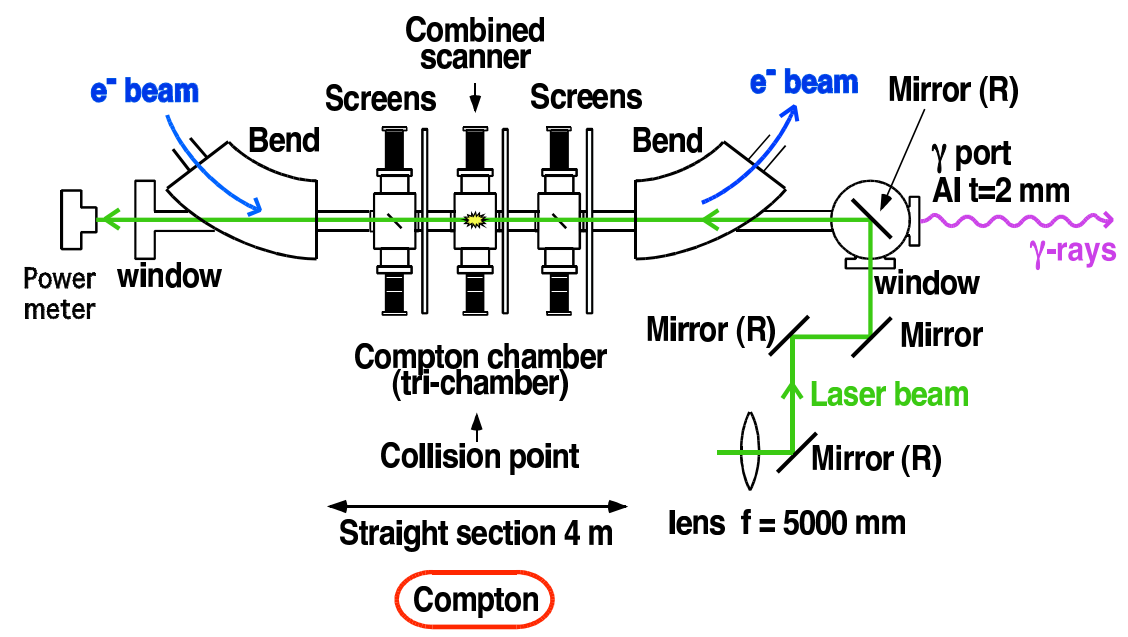

Figure 5.8: Compton chamber: with screens at the collision point as well as upstream and downstream of the collision point, the positions and angles of the laser and electron beams are adjusted so that accurate head-on collisions can be realized. The spot sizes of the electron and laser beams are measured with a wire and a knife-edge scanner.

for the parallel and the anti-parallel cases are measured downstream of magnetized iron. Here the parallel (anti-parallel) case means that the spins of the photons and the electrons in the magnetized iron are parallel (anti-parallel) to each other. Since no event identification is necessary in this transmission method, it is suitable for measuring the polarization of a high-intensity beam in which a very large number of photons in the beam interact in a very short time ( $30 \mathrm{psec})$. The iron pole in an electromagnet is used as the magnetized iron. The polarity of the magnet is flipped to measure the transmission asymmetry in the two cases. The transmission asymmetry is proportional to the polarization of the photons. Fig 5.9 shows the measured transmission asymmetry of the photons [202]. This asymmetry corresponds to a photon polarization of about $90 \%$. Photons are measured above a threshold of $21 \mathrm{MeV}$. 
(a)

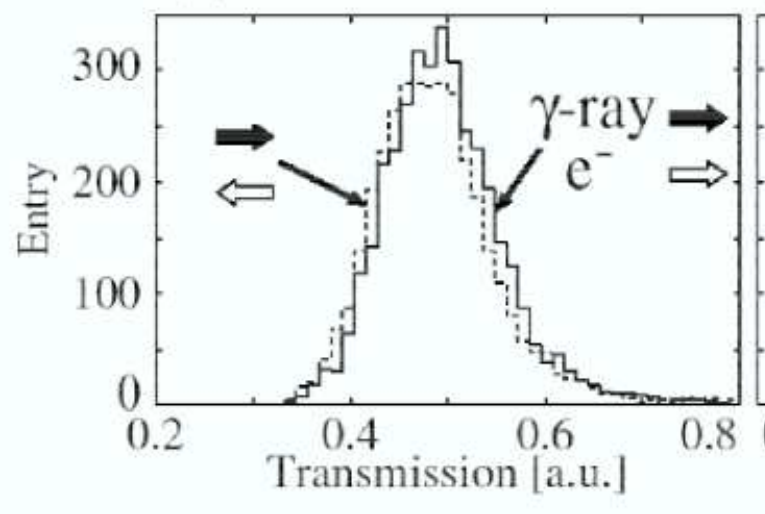

$$
\begin{array}{ll}
\mathbf{A}=\mathbf{- 0 . 9 3} \pm \mathbf{0 . 1 5} \% & \mathbf{A}=\mathbf{1 . 1 8} \pm \mathbf{0 . 1 5} \% \\
\text { laser pol. }=-79 \% & \text { laser pol. }=+79 \%
\end{array}
$$

Figure 5.9: Measured transmission asymmetry of the photons [202]. This asymmetry is proportional to the polarization of the photons and corresponds to a photon polarization of about $90 \%$. The photons are measured above a threshold of $21 \mathrm{MeV}$.

Fig. 5.10 shows the apparatus for producing polarized positrons from polarized photons and for measuring the polarization of these positrons. In this design, $\sim 10^{7}$ gamma-

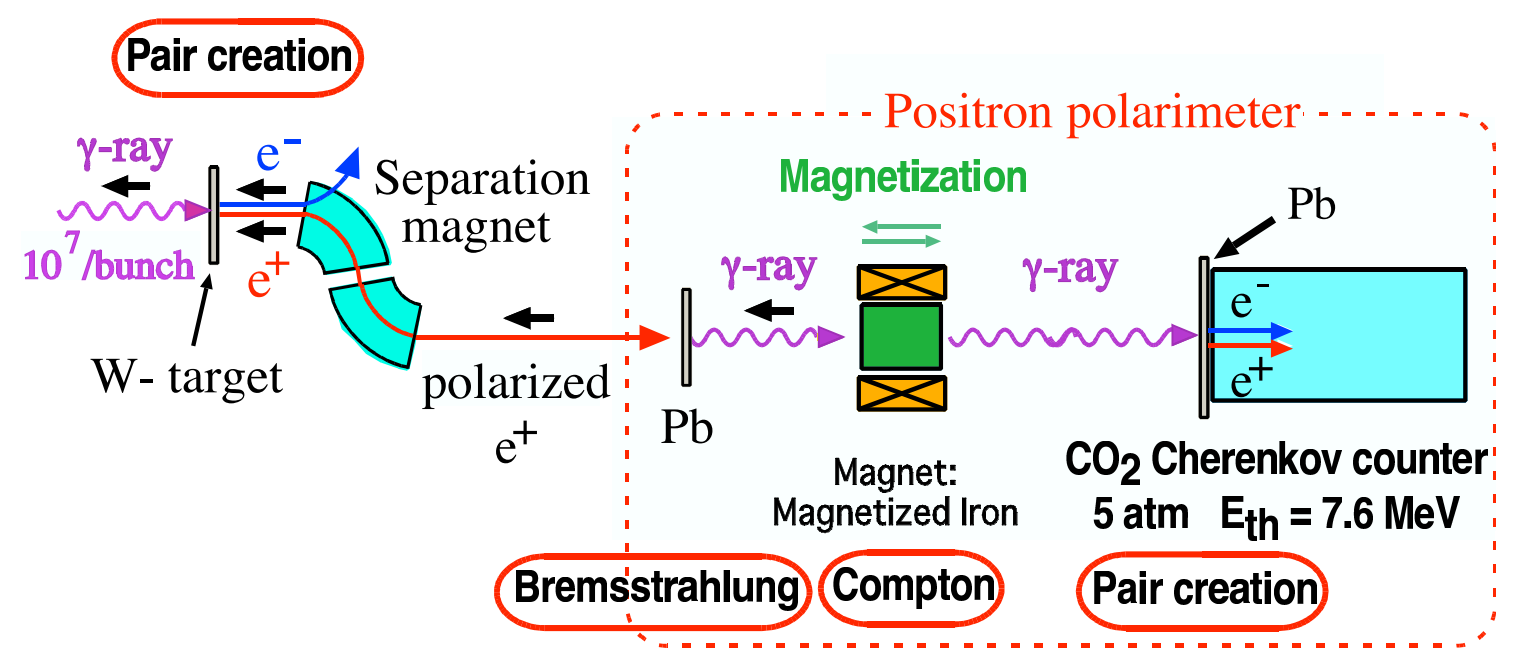

Figure 5.10: Production of polarized positrons in a thin tungsten target. Dipole magnets separate the polarized electrons and positrons. The positron polarization is measured with the Compton-transmission method.

photons are produced in a bunch. Polarized electrons and positrons are produced by pair creation in a thin tungsten target. A pair of dipole magnets separates positrons and electrons. The dipole magnets select only the high-energy part of the positron spectrum (about $25-45 \mathrm{MeV}$ ). The number of positrons after momentum selection is $\sim 10^{4}$ per 
bunch. One uses again a Compton-transmission method to measure the positron polarization, fig. 5.10. The expected value of the transmission asymmetry is about $0.7 \%$. The experiment is in progress.

\subsection{Polarized electrons}

Highly-polarized electrons are obtained from GaAs by directing a circularly-polarized laser beam on to a thin (typically $100 \mathrm{~nm}$ ) p-doped epilayer of strained GaAs [203], of strained InGaAs [204], and of a short period superlattice [205]. Only a small fraction of the photons are absorbed in the epilayer. Electrons with zero momentum are promoted from the highest level of the valence band (VB) to the lowest level of the conduction band (CB) upon the absorption of laser photons, see fig. 5.11. A biaxial compressive strain produced by a lattice mismatch with the substrate or by the quantum confinement associated with short-period superlattice (SL) structures breaks the degeneracy of the heavy-hole (hh) and light-hole (lh) bands at the highest level of the VB [209]. A hh-lh separation of $\sim 80 \mathrm{MeV}$ is readily achieved, which in carefully grown structures, is sufficient to allow the selection of electrons from the hh band only. With the laser wavelength tuned to the band gap, angular momentum selection rules result in the production of CB electrons in one spin state exclusively. A thin epilayer is chosen to minimize strain relaxation. As the $\mathrm{CB}$ electrons diffuse to the surface, they undergo some depolarization, primarily by interaction with holes. This effect can be considerably reduced by decreasing the dopant density [206] (everywhere except the last few nanometers near the surface, which is often called gradient doping method [207]). Near the surface, the energy levels for p-doped GaAs bend downwards. Most of the electrons reaching the surface are confined to this bandbending region (BBR) for a finite time until they are emitted to vacuum or lose sufficient energy for them to be trapped in surface states. Although the BBR is depleted of holes, the confined but still mobile electrons in the BBR lose energy by scattering from optical phonons, as a result of which the amplitude and phase of the spin precession vector is continually reoriented, leading to a significant depolarization. The probability for electrons to escape to vacuum can be as high as $20 \%$ if the surface is properly activated with caesium and an oxide to create a negative electron affinity.

The SLC demonstrated that electron beams with polarization at the IP approaching $80 \%$ can be generated reliably for a period of years. The SLC beam was produced using a 100-nm thick GaAsP/GaAs strained-layer photocathode. By decreasing the dopant density in the bulk, the polarization of this type of cathode was slightly improved for the initial run of a parity violation experiment E158 at SLAC. Higher polarization is an ongoing R\&D aim. Several laboratories have reported electron beams with polarization of $90 \%$ or even higher. The problem is that such high values are universally achieved only with a cathode surface having a relatively low quantum efficiency (QE), defined as the number of photoemitted electrons per incident photon. Improvements in polarization while maintaining a high QE have recently been achieved with strained GaAsP/GaAs superlattice (SL) structures [208]. Each layer of the superlattice (typically $4 \mathrm{~nm}$ ) is considerably thinner than the critical thickness $(\sim 10 \mathrm{~nm})$ for the onset of strain relaxation, while the transport efficiency for electrons in the conduction band can still be high. In addition the effective band gap for such superlattices is larger than for GaAs alone, which improves the QE. Today, 100-nm thick, gradient-doped GaAsP/GaAs superlattice pho- 
(a) GaAs
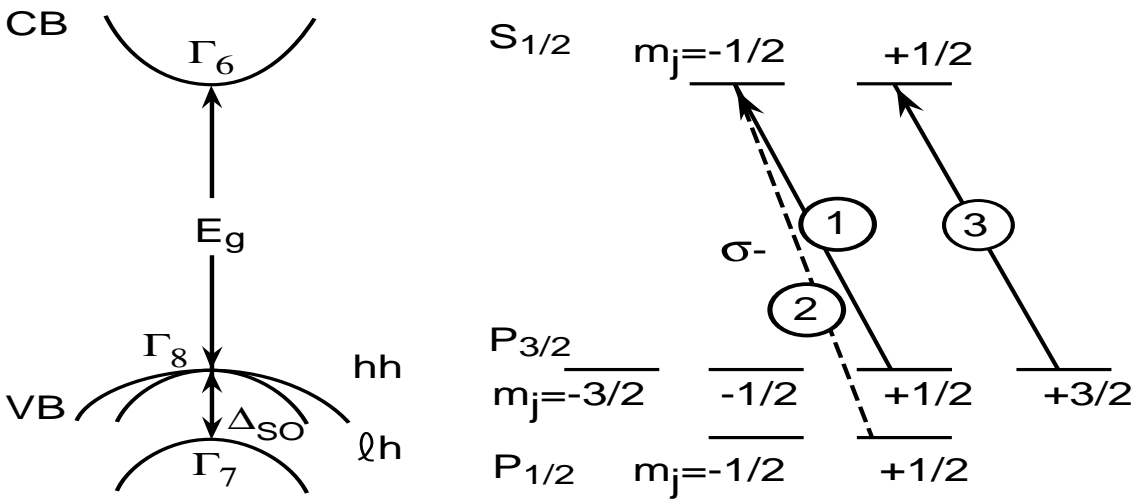

(b) GaAsP/GaAs Strained-Layer
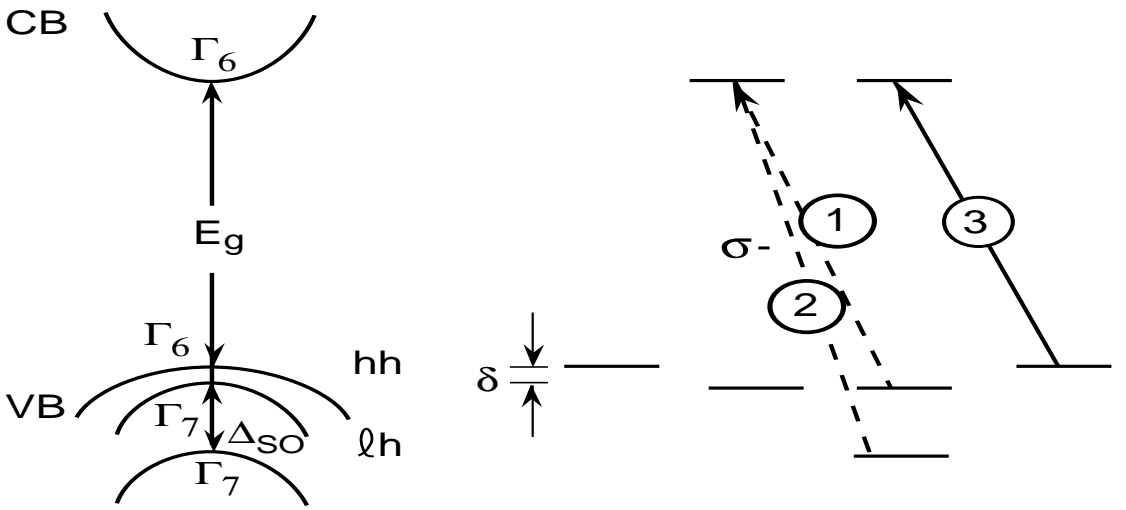

Figure 5.11: Band gap diagram and transition probabilities at the $\Gamma$ point for (a) GaAs and (b) strained-layer GaAsP/GaAs. $\Delta_{\mathrm{SO}}$ is the spin-orbit splitting, $\delta$ is the hh-lh (heavy holelight hole) splitting due to strain. A non-zero $\delta$ can also be produced with a superlattice structure. At room temperature, $\mathrm{E}_{g}=1.424 \mathrm{eV}, \Delta_{\mathrm{SO}}=0.340 \mathrm{eV}$. On the right, relative transition probabilities between $\mathrm{s}$ and $\mathrm{p}$ orbitals for excitation by $\sigma^{-}$light are shown. Transitions at the band gap minimum are shown as solid lines.

tocathodes routinely yield at least $85 \%$ polarization at low energy with a maximized QE of $\sim 1 \%$. This type of cathode was successfully used during the summer 2003 dedicated run at SLAC of E158-III, for which the polarization at high energy, measured by a Møller polarimeter, was $(89 \pm 4) \%$ except for short periods following refreshment of the cathode QE (accomplished every few days by adding a small amount of Cs to the surface). This experience points to the possibility of a constant $90 \%$ polarization if a technique to control the minimum surface barrier can be developed. Recent data for 2 SL photocathodes are compared with that for the best of the strain-layer cathodes in table 5.3. In the table, $\lambda_{0}$ is the wavelength corresponding to the maximum polarization, $P_{e, \max }$. The absolute accuracy of the polarimeters is of the order of $\pm 5 \%$.

The ILC beam is a train of 2820 bunches spaced 337 ns apart at a repetition rate of $5 \mathrm{~Hz}$. The electron source should be able to generate at least twice the charge required at the interaction point (IP). Thus the required charge at the source will be $\sim 6.4 \mathrm{nC}$ per bunch, $\sim 20 \mu \mathrm{C}$ per train. A dc-biased photocathode gun as used for the SLC is expected to be used. The bunching systems necessary for the ILC electrons are very similar to those used at the SLC notwithstanding the requirement of the ILC bunch format. 


\begin{tabular}{|cccrrrrr|}
\hline $\begin{array}{c}\text { Cathode } \\
\text { Structure }\end{array}$ & $\begin{array}{c}\text { Growth } \\
\text { Method }\end{array}$ & Polarimeter & $P_{e, \max }$ & $\begin{array}{r}\lambda_{0} \\
(\mathrm{~nm})\end{array}$ & $Q E_{\max }\left(\lambda_{0}\right)$ & Ref. \\
\hline $\begin{array}{c}\text { GaAsP/GaAs strained SL: } \\
\text { Nagoya }\end{array}$ & MOCVD & Mott & $\geq 0.90$ & 775 & 0.004 & {$[210]$} \\
SLAC & MBE & CTS Mott & 0.86 & 783 & 0.012 & {$[211]$} \\
& & Møller E158-III & 0.89 & 780 & 0.008 & {$[212]$} \\
GaAsP/GaAs strained-layer: & & & & & & \\
SLAC & MOCVD & CTS Mott & 0.82 & 805 & 0.001 & {$[213]$} \\
& & Møller E158-I & 0.85 & 800 & 0.004 & {$[214]$} \\
\hline
\end{tabular}

Table 5.3: Comparison of 2 strained superlattice and 1 strained-layer photocathodes.

\subsection{Spin manipulation systems}

A spin rotation scheme for the ILC that allows the polarization vector of the electron and positron beams to be tuned independently for two interaction regions (IR) is described in [215]. The correct polarization direction for a particular IR can be selected by directing the beam into one of two parallel spin rotation beam lines located between the damping ring and the linac. With fast kicker magnets, it is possible to rapidly switch between these parallel beam lines, so that polarized beams can be delivered to two IRs on a pulse-train by pulse-train basis. Fig. 5.12 shows the layout of the electron damping ring system and fig. 5.13 shows the layout for the positron damping ring system. A similar scheme can be employed in the low-energy transport (LTR) to the positron damping ring, to allow rapid helicity switching for polarized positrons. In that case the axial solenoid fields must have equal but opposite directions in the two lines. A pair of kicker magnets is turned on between pulse trains to deflect the beam to the spin rotation solenoids with negative B-field.

The system shown in fig. 5.14 forms the basic spin rotation building block in each of the IR1/IR2 ring-to-linac transport lines.

If properly designed, such a spin rotation system after the damping ring will allow the spin of the beams to be set to any arbitrary orientation by the time they reach the IP. The system described in [216] accomplishes the desired spin manipulation while minimizing emittance dilution of the flat beams. Fig. 5.14 illustrates the basic features of the system which consists of two solenoidal rotation systems with a bend rotation system in between. Starting with an initial vertical spin vector coming out of the damping ring, the final spin vector at the exit of the full system is $\vec{S}$ [216],

$$
\vec{S}=\stackrel{\leftrightarrow}{\Omega}_{\text {tot }} \cdot\left(\begin{array}{r}
0 \\
\pm 1 \\
0
\end{array}\right)=\left(\begin{array}{c}
\mp \sin \phi_{1} \cos \phi_{2} \\
\pm \cos \phi_{1} \cos \phi_{2} \\
\pm \sin \phi_{2}
\end{array}\right)
$$

where $\phi_{1}$ and $\phi_{2}$ are the spin rotations due to the first and second solenoidal rotation systems. From eq. (5.1), it is seen that any arbitrary orientation of the spin vector is possible through a suitable choice of $\phi_{1}$ and $\phi_{2}$. 


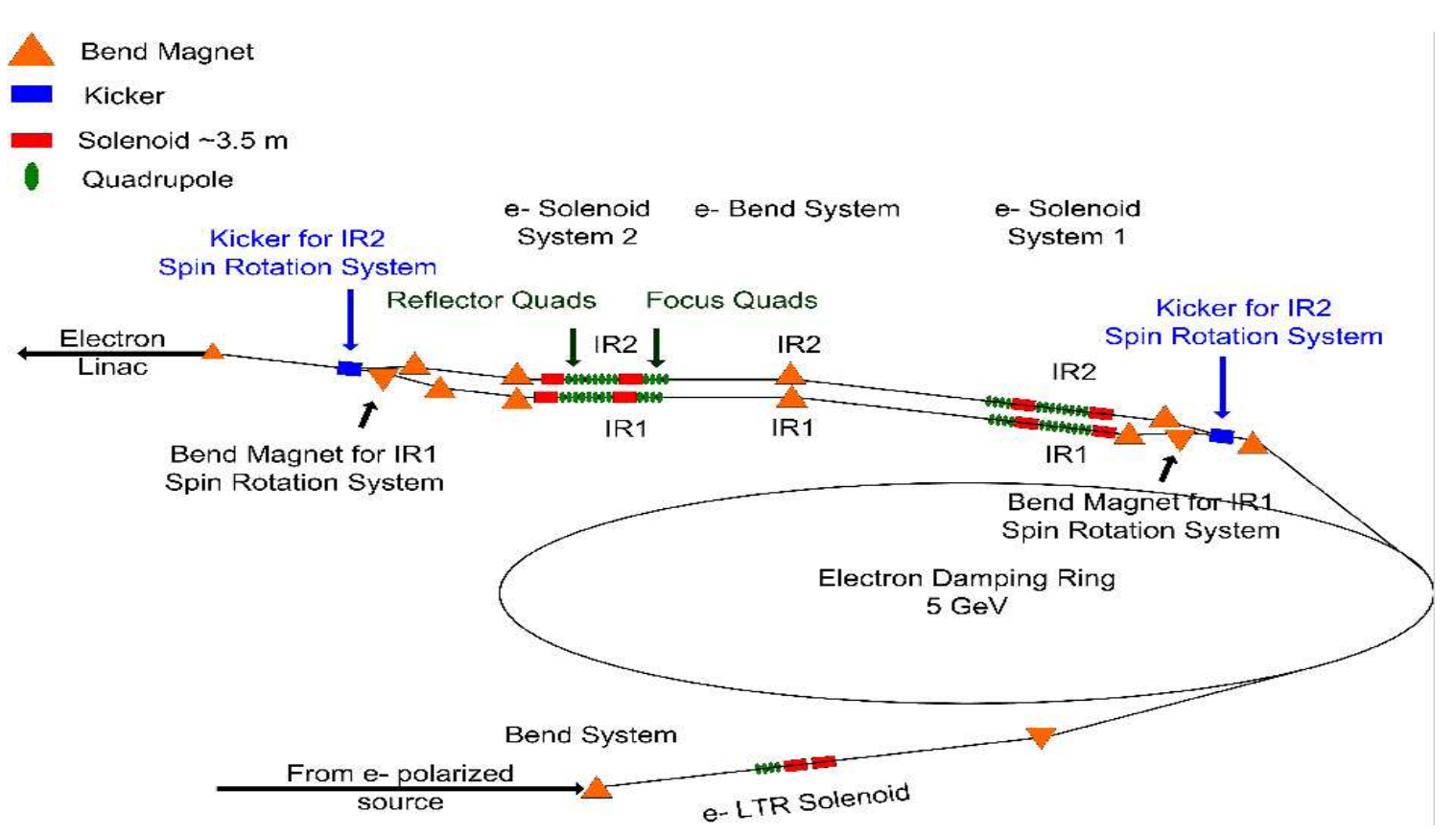

Figure 5.12: Layout of the electron damping ring system showing the parallel spin rotation beam lines for IR1 and IR2. A pair of kicker magnets is turned on between pulse trains to deflect the beam to the spin rotation solenoids for IR2 [215].

Each solenoid system is made up of an initial solenoid followed by an optical transport section which gives a unity transformation in the horizontal plane and a -1 transformation in the vertical plane, an optical reflection. The optical section is followed by a second solenoid. Each solenoid is set to provide a spin rotation of half the total required for the particular solenoidal system, $\phi_{i}$. The optical reflection transport is required to avoid transverse betatron coupling of the beam by cancelling the rotation due to the solenoids.

The bend section has a constant spin rotation contribution of $\pi / 2$ in the horizontallongitudinal plane; the spin rotates about the longitudinal axis in the solenoids. Upstream of the damping rings, emittance dilution is not of concern in the longitudinal to vertical spin transformation. This system consists of a simple bend followed by a solenoid of suitable strength. 


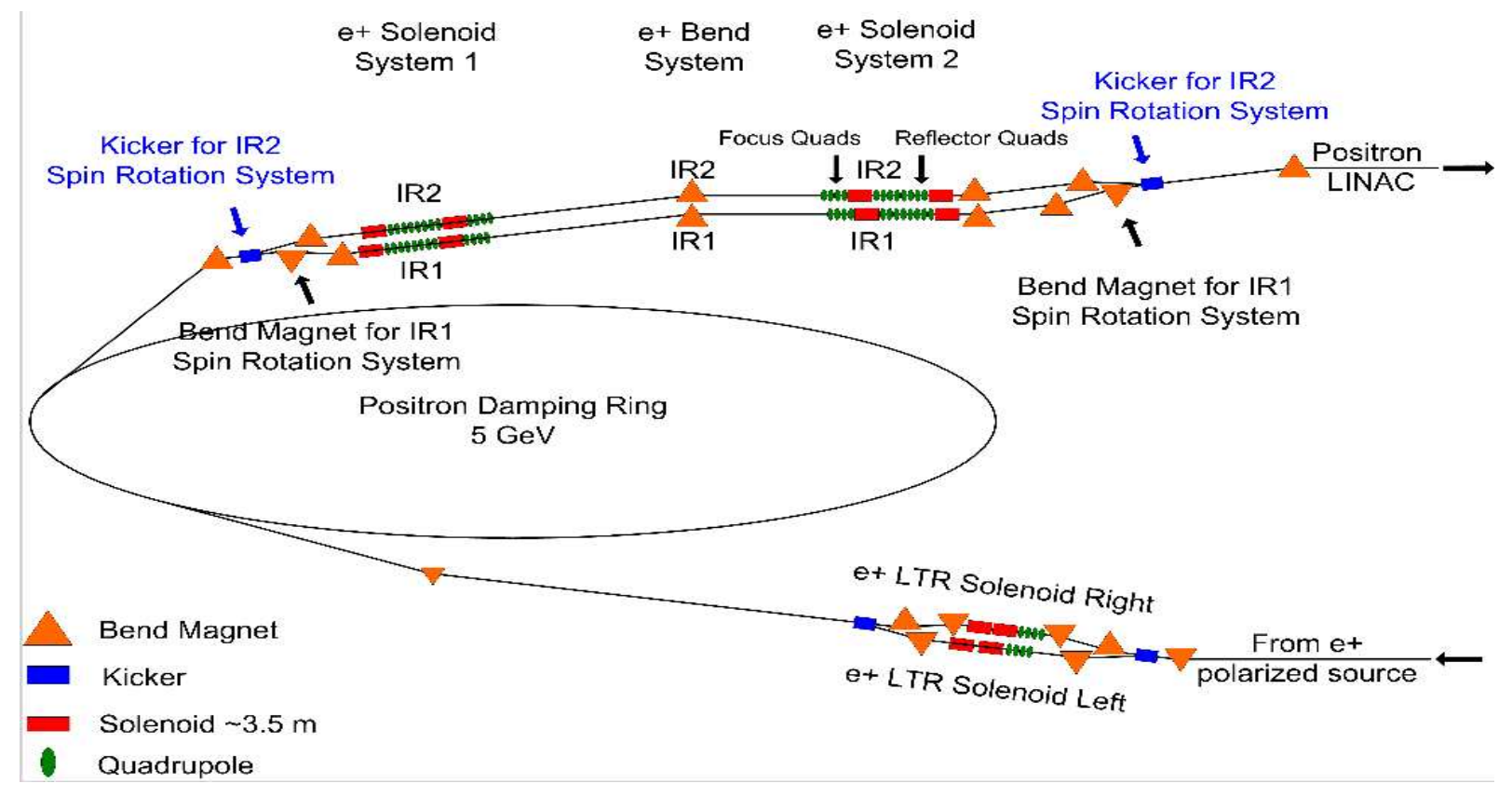

Figure 5.13: Layout of the positron damping ring system showing the parallel spin rotation beam lines for randomly selecting the positron polarization direction. A pair of kicker magnets is turned on between pulse trains to deflect the beam to the spin rotation solenoids with negative B field [215]. This is obviously not necessary with electrons whose polarization is switched at the source.

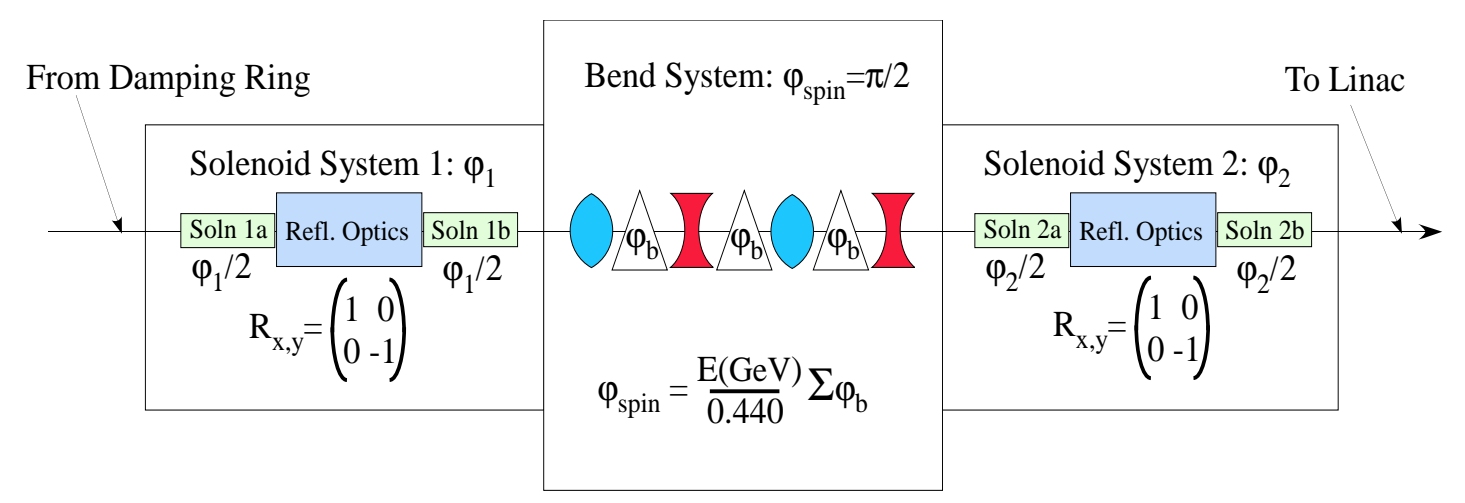

Figure 5.14: Spin rotation system to rotate a vertical spin coming from the damping ring to an arbitrary spin orientation. See text for explanation. 


\subsection{Polarimetry at the ILC}

The primary polarimeter measurement at the ILC will be performed by a Compton polarimeter. An accuracy of $\left(\Delta P_{e^{-}} / P_{e^{-}}\right)=0.25 \%$ should be achievable [53]. The polarimeters can be located upstream or downstream of the Interaction Point (IP) and it is desirable to implement polarimeters at both locations. The downstream polarimeter may require a large crossing angle at the IP; polarimetry is one of many issues to be considered in a decision on the IR crossing angles.

Compton polarimetry is chosen as the primary polarimetry technique for several reasons:

- The physics of the scattering process is well understood in QED, with radiative corrections less than $0.1 \%$ [217];

- Detector backgrounds are easy to measure and correct for by using laser off pulses;

- Polarimetry data can be taken parasitic to physics data;

- The Compton scattering rate is high and small statistical errors can be achieved in a short amount of time (sub-1\% precision in one minute is feasible);

- The laser helicity can be selected on a pulse-by-pulse basis;

- The laser polarization is readily determined with $0.1 \%$ accuracy.

\subsubsection{Møller and Bhabha polarimetry}

A Møller or Bhabha scattering polarimeter can be considered as a cross check of the principle high-energy Compton polarimetry, or at various intermediate stages of the acceleration process. This well-known method has been applied in numerous experiments [218220] with energies ranging from MeVs to multi-GeVs. Møller type polarimeters have in particular been used for many years in conjunction with fixed-target experiments at SLAC [221-226] and elsewhere [227-230].

The application of this technique at linear collider beams appears to be generally inferior, at least in its conventional incarnations, in comparison with laser-based Compton polarimetry.

Møller polarimetry at a linear collider has currently still some disadvantages with respect to Compton polarimetry:

1. low target polarization: only about $8 \%$ electron polarization is possible with ferromagnetic materials (to be compared with $100 \%$ laser polarization in Compton polarimetry) and it is not easy to calibrate this important quantity with great accuracy;

2. limited precision: only one single Møller polarimeter with rather exceptional properties has reported a precision $\Delta P / P=0.5 \%$ [230,231]. The typical performance of more conventional devices has been only at the level of $2-5 \%$ or worse [221-229];

3. invasive: insertion of the target is invasive to the beam, i.e. such a polarimeter can only operate in the dumpline of the beam, or only intermittently, if used upstream of the collider interaction point. 
Additional issues that have to be considered are beam heating of the target and multiple event topologies [232].

Since all of the major drawbacks of the conventional Møller technique originate in the ferromagnetic foil target, one may wonder if not a radically different target with nearly $100 \%$ electron polarization is possible, which furthermore should be thin enough to preserve the pristine quality of the beam.

A polarized gas target with similar properties has in fact been in operation since several years in the HERA storage ring at the HERMES experiment [233], and Møller and Bhabha polarimetry has routinely been employed to diagnose the performance of the spin-polarized target cell [234]. However, since the average $e^{+} / e^{-}$beam currents of HERA are $1000 \times$ larger than those at the linear collider, it is doubtful if something like the HERMES gas target could be developed to be suitable for polarimetry at the ILC.

One could summarize that dedicated Møller/Bhabha beam polarimetry based on conventional target technology is generally inferior to laser-based Compton polarimetry, but may be useful in limited precision applications at intermediate energies. With an advanced target design, an ultimate precision $\Delta P / P=0.5 \%$ may be achievable and useful as an occasional crosscheck of the principle Compton results, but the general operational drawbacks remain.

\subsubsection{Compton scattering basics}

One defines $E_{0}$ and $\omega_{0}$ to be the incident energies of the electron and photon, and $E$ and $\omega$ to be the scattered energies of the electron and photon. The dimensionless $x, y$ and $r$ scattering parameters are defined by:

$$
\begin{aligned}
x & =\frac{4 E_{0} \omega_{0}}{m^{2}} \cos ^{2}\left(\theta_{0} / 2\right) \simeq \frac{4 E_{0} \omega_{0}}{m^{2}} \\
y & =1-\frac{E}{E_{0}}=\frac{\omega}{E_{0}} \\
r & =\frac{y}{x(1-y)}
\end{aligned}
$$

where $m$ is the mass of the electron and $\theta_{0}$ is the crossing angle between the electron beam and the laser beam. For polarimeters with small crossing angles at the Compton IP, $\cos ^{2}\left(\theta_{0} / 2\right) \simeq 1$

The spin-dependent differential Compton cross section is given by:

$$
\begin{aligned}
\left(\frac{d \sigma}{d y}\right)_{\text {Compton }} & =\left(\frac{d \sigma}{d y}\right)_{\text {unpol }}\left[1+P \lambda A_{z}(x, y)\right] \\
\left(\frac{d \sigma}{d y}\right)_{\text {unpol }} & =\frac{0.499 \mathrm{barn}}{x}\left[\frac{1}{1-y}+1-y-4 r(1-r)\right] \\
A_{z}(x, y) & =r x(1-2 r)(2-y)
\end{aligned}
$$

where $P$ is the longitudinal polarization of the electron and $\lambda$ is the circular polarization of the laser photon. The Compton asymmetry analyzing power, $A_{z}(x, y)$, is maximal at the kinematic endpoint, corresponding to $180^{\circ}$ backscattering in the center-of-mass frame, 


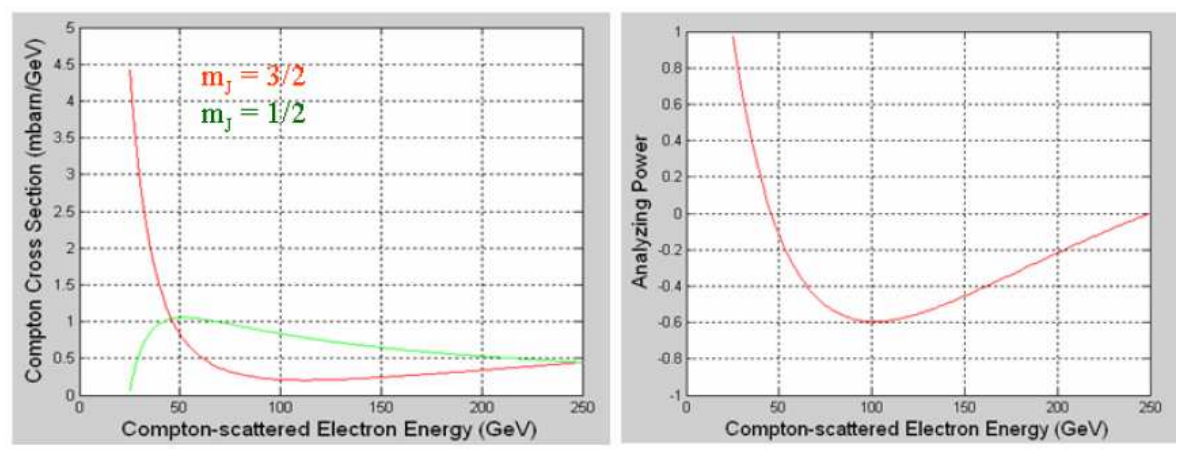

Figure 5.15: Compton cross section for scattering of $532 \mathrm{~nm}$ photons with a $250 \mathrm{GeV}$ electron beam. The $J_{z}=3 / 2\left(J_{z}=1 / 2\right)$ cross section for electron and photon spins aligned (anti-aligned) is shown in red (light green).

with

$$
E_{\min }=E_{0} \frac{1}{1+x}
$$

For a $250 \mathrm{GeV}$ electron beam colliding with a 532-nm laser, the Compton-scattered electrons have their kinematic endpoint at $E_{\min }=25.1 \mathrm{GeV}$ with an analyzing power $A_{z}=$ $98 \%$. Figure 1 shows the resulting $J_{z}=3 / 2$ and $J_{z}=1 / 2$ Compton cross sections and analyzing power.

\subsubsection{Upstream Compton polarimeter}

The Compton Polarimeter upstream of the collider IP requires the Compton IP be just upstream of the energy collimation region so that off-energy Compton electrons are not lost in the Interaction Region. An additional requirement is that the beam trajectory at the Compton IP be parallel to the trajectory at the collider IP. A polarimeter satisfying these requirements was included in the TESLA design and we provide a brief description here [235].

The Compton IP is located 630 meters upstream of the collider IP, see Fig. 5.16. The Compton laser is pulsed with a pattern that matches the pulse and bunch structure of the ILC. In this way it is possible to achieve high luminosity, typically six orders of magnitude higher than with continuous lasers of comparable average power.

Compton electron detection in the multi-event (integrating) mode is the principal detection method. Event rates and statistical errors have been calculated for different beam energies and laser parameters [235]. Table 5.4 gives typical polarimeter parameters for $\sqrt{s}=500 \mathrm{GeV}$. The performance is similar for other energy regimes. For much higher or lower beam energies, it will be advantageous to change the wavelength of the laser or to adapt a four magnet chicane around the Compton IP comparable to the one of the downstream polarimeter, in order to retain maximum coverage of the electron detector hodoscope.

\subsubsection{Downstream Compton polarimeter}

The design criteria for an extraction line polarimeter may prevent a downstream polarimeter if there is no crossing angle at the IP. The 20-mrad crossing angle design con- 


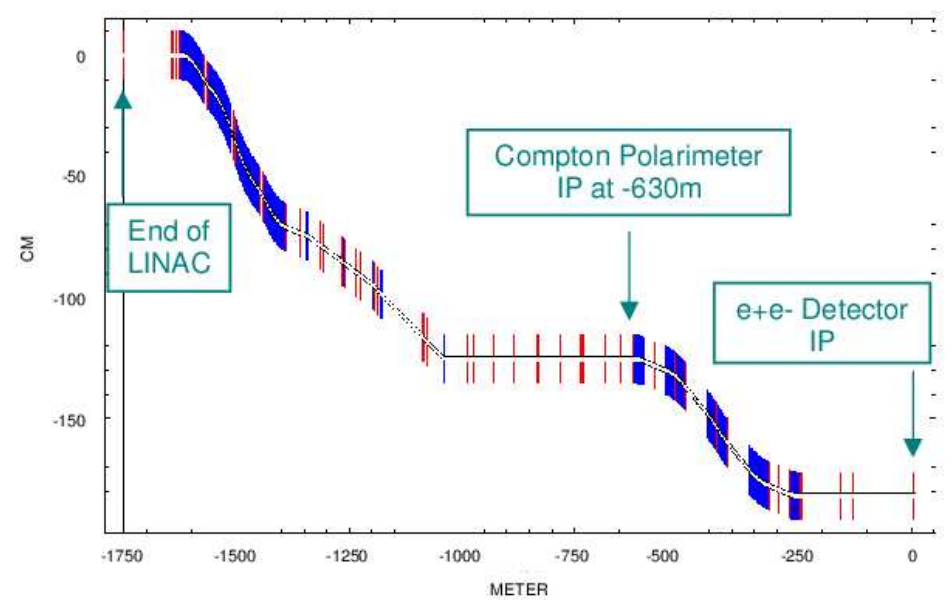

Figure 5.16: Upstream Compton polarimeter in the TESLA design.

\begin{tabular}{|lccc|}
\hline & $e^{+} / e^{-}$beam & $\begin{array}{c}\text { Upstream } \\
\text { laser beam }\end{array}$ & $\begin{array}{c}\text { Downstream } \\
\text { laser beam }\end{array}$ \\
\hline Energy & $250 \mathrm{GeV}$ & $2.3 \mathrm{eV}$ & $2.3 \mathrm{eV}$ \\
Charge or energy/bunch & $2 \cdot 10^{10}$ & $35 \mu \mathrm{J}$ & $100 \mathrm{~mJ}$ \\
Bunches $/$ sec & 14100 & 14100 & 5 \\
Bunch length $\sigma_{t}$ & $1.3 \mathrm{ps}$ & $10 \mathrm{ps}$ & $1 \mathrm{~ns}$ \\
Average current(power) & $45 \mu A$ & $0.5 \mathrm{~W}$ & $0.5 \mathrm{~W}$ \\
$\sigma_{x} \cdot \sigma_{y}(\mu \mathrm{m})$ & $10 \cdot 1$ upstream & $50 \cdot 50$ & $100 \cdot 100$ \\
& $30 \cdot 60$ downstream & & \\
\hline & & Upstream & Downstream \\
& & polarimeter & polarimeter \\
Beam crossing angle & $10 \mathrm{mrad}$ & $11.5 \mathrm{mrad}$ \\
Luminosity & & $1.5 \cdot 10^{32} \mathrm{~cm}^{-2} \mathrm{~s}^{-1}$ & $5 \cdot 10^{30} \mathrm{~cm}^{-2} \mathrm{~s}^{-1}$ \\
Event rate at 25-GeV Endpoint & & $300,000 / \mathrm{GeV} / \mathrm{sec}$ & $10,000 / \mathrm{GeV} / \mathrm{sec}$ \\
$\Delta P / P$ stat. error & $<1 \% / \mathrm{sec}$ & $<1 \% / \mathrm{min}$ \\
$\Delta P / P$ syst. error & & $0.25 \%$ & $0.25 \%$ \\
\hline
\end{tabular}

Table 5.4: Compton polarimeter parameters at $250 \mathrm{GeV}$.

sidered for NLC, however, permitted a downstream polarimeter and we give a brief description for it here with beam parameters appropriate for the ILC design.

The Compton IP is located approximately 60 meters downstream from the collider IP (see Figure 5.17). [236] The Compton IP is at a secondary focus in the middle of a chicane with $20 \mathrm{~mm}$ dispersion, but with no net bend angle with respect to the $e^{+} e^{-}$collision IP. Beam losses in the extraction line are acceptable, both for machine protection and for ILC detector backgrounds.

The Compton laser has a low repetition rate of $5-10 \mathrm{~Hz}$, but has high power to give good signal-to-background ratio in the more difficult downstream environment with disrupted primary electrons and beamstrahlung photons present. The Compton laser pulses collide with a small fraction of the $14 \mathrm{kHz}$ electron bunch rate, but the timing of the Compton laser pulses can be varied so as to sample systematically all electron bunches in the train and determine any variation of the beam polarization along the train. 


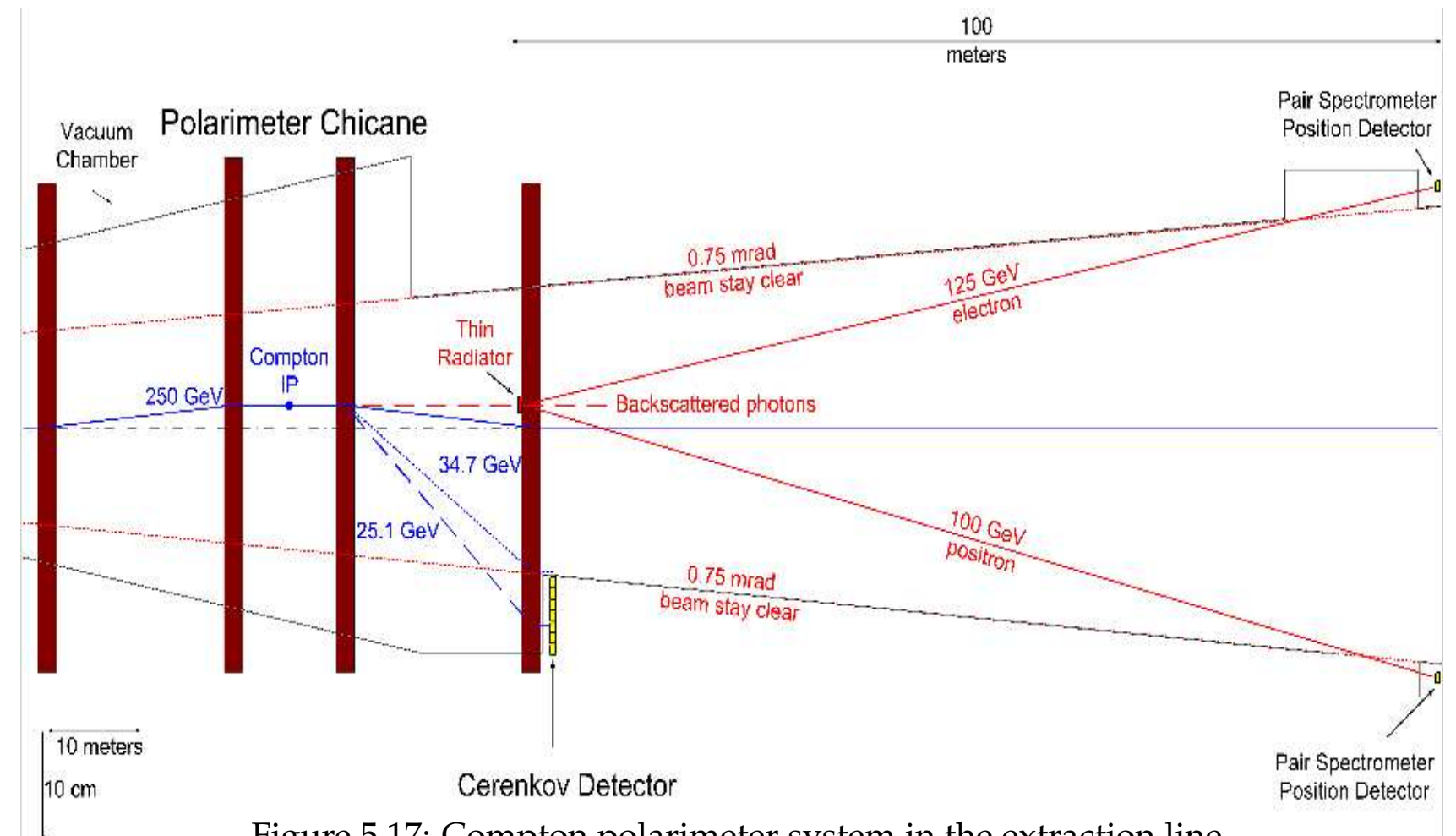

Figure 5.17: Compton polarimeter system in the extraction line.

The primary Compton detector is envisioned to be a segmented electron detector operating in the multi-electron (integrating) mode, sampling the electron flux at energies near the Compton kinematic edge at $25.1 \mathrm{GeV}$ (for $250 \mathrm{GeV}$ primary electron energy and $532 \mathrm{~nm}$ laser photons). The Compton electron detector must discern between the Compton edge electrons and low energy disrupted primary electrons, and it must be located outside a $0.75-\mathrm{mrad}$ cone from the IP that contains the intense flux of beamstrahlung photons. Figure 5.18 shows the y distribution of $25.1-\mathrm{GeV}$ Compton-scattered electrons at the detector located downstream of the polarimeter chicane magnets. The Comptonedge electrons peak at $\approx 18 \mathrm{~cm}$, well separated from the tails of the disrupted electron beam. For polarimeter operation at electron beam energies other than $250 \mathrm{GeV}$, the chicane dipoles should retain the same B field. This changes the dispersion at the Compton IP, but since the Compton edge endpoint energy does not change quickly with incident electron beam energy, the location of Compton-edge electrons at the Compton detector plane will only shift slightly. The parameters for the downstream Compton polarimeter are summarized in Table 5.4. In that context one should note that due to the strong fields significantly higher depolarization effects are expected at the possible multi-TeV LC design [183], so that a downstream polarimeter might be indispensable in that case.

\subsubsection{Luminosity-weighted polarization}

The luminosity-weighted beam polarization will differ from the measured polarization due to disruption and radiation in the beam-beam collision process. There are also effects from polarization spread and spin transport.

The spin motion of a deflected electron or positron beam in a transverse magnetic field 

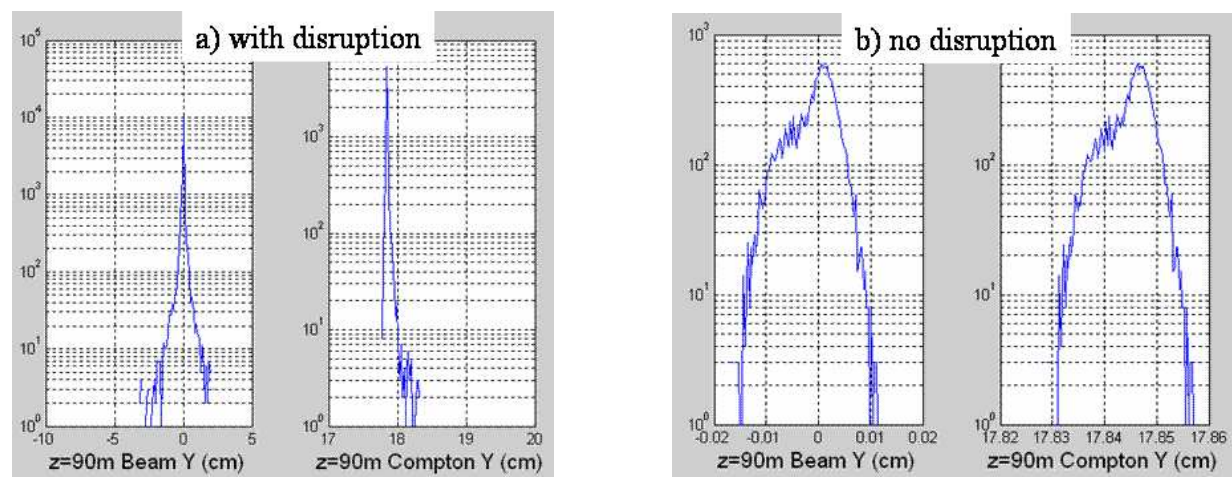

Figure 5.18: Vertical distributions of disrupted beam and Compton-edge electrons at the Compton detector plane a) with $e^{+} e^{-}$collisions and b) without collisions.

follows from the familiar T-BMT expression

$$
\theta^{\text {spin }}=\gamma \frac{g-2}{2} \theta^{\text {orbit }}=\frac{E_{0}}{0.44065 G e V} \theta^{\text {orbit }}
$$

where $\theta^{\text {orbit }}$ and $\theta^{\text {spin }}$ are the orbit deflection and spin precession angles, $E_{0}$ is the beam energy, $\gamma=E_{0} / m$, and $(g-2) / 2$ is the famous g-factor anomaly of the magnetic moment of the electron.

The difference between the luminosity-weighted beam polarization and the polarimeter measurement is written as $d P=P_{z}^{l u m-w t}-P_{z}^{C I P}$. To minimize $d P$, it is required that the beam direction at the IP of the polarimeter (Compton IP) be aligned with the collision axis at the $e^{+} e^{-}$IP to within $50 \mu \mathrm{rad}$. We consider estimates of several contributions to $d P$, assuming full longitudinal spin alignment at the polarimeter IP. The use of both an upstream and a downstream polarimeter will assist in achieving the desired small $d P$ and estimating the associated systematic error [237].

The contributions to $d P$ for the downstream polarimeter are considered first, using estimates in the NLC design study [237]. Orbit misalignments between the polarimeter IP and the collision IP are expected to be below $50 \mu \mathrm{rad}$, which would give $d P=-0.04 \%$. Imperfect compensation for steering effects due to the angle between the beam trajectory and the detector solenoid is expected to give an additional trajectory alignment uncertainty of $30 \mu \mathrm{rad}$, and gives a contribution $d P=-0.01 \%$. The effect of SokolovTernov spin flips is expected to contribute $d P=+0.3 \%(d P=-0.1 \%)$ for downstream polarimeter measurements with (without) collisions. The angular divergence of the incoming beam is expected to contribute $d P=-0.03 \%$, while the angular divergence of the outgoing beam is expected to contribute $d P=+0.1 \%(d P=-0.25 \%)$ for downstream polarimeter measurements with (without) collisions. Lastly, effects from chromatic aberrations, which were important at SLC, are expected to be negligible. Adding all these contributions together, we expect a total difference $d P=+0.32 \%(d P=-0.43 \%)$ for downstream polarimeter measurements with (without) collisions.

A similar compilation of these effects for an upstream polarimeter leads to $d P=$ $-0.42 \%$ [237]. The systematic errors associated with $d P$ should be substantially smaller than the size of $d P$, and having independent information from 3 polarimeter measurements (upstream, downstream with and without collisions) will be important in minimizing this. 


\subsubsection{Transverse Polarimetry}

The spin rotation system with two spin rotators following the Damping Ring can achieve arbitrary spin orientation in the linac or at the IP as described in section 5.4. Eq. 5.1 describes the spin vector at the beginning of the main linac. A unitary spin transport matrix, $R$, then describes spin transport from that location to the IP or polarimeters. To determine $R$ using the longitudinal Compton polarimeter one performs a 3-state measurement by choosing appropriate spin rotator settings to align the electron spin along the $x, y$, or $z$ axis at the start of the main linac. The $z$-component of the spin transport matrix can be measured with the Compton polarimeter, which measures the longitudinal electron polarization,

$$
P_{z}^{C}=R_{z x} \cdot P_{x}^{L}+R_{z y} \cdot P_{y}^{L}+R_{z z} \cdot P_{z}^{L} .
$$

The three $P_{z}^{C}$ measurements for each $x, y$, or $z$ spin orientations at the start of the main linac determines the spin rotation matrix elements $R_{z x}, R_{z y}$, and $R_{z z}$. This is sufficient to determine the full rotation matrix, which is described by three Euler angles. The matrix $R$ can be inverted to determine the required spin rotator settings for the desired spin orientation at the IP.

When running transverse polarization at the IP, the longitudinal Compton polarimeter can monitor that the longitudinal beam polarization stays close to zero. Calibrations of the beam polarization can be done periodically (perhaps every 1-2 days, depending on stability and required precision) by changing the spin rotator settings to achieve longitudinal polarization. The ILC beam polarization is likely to be fairly stable and well understood and this method should achieve a precision of about $1 \%$. Another possibility is to use the transverse asymmetries in $\mu^{+} \mu^{-}$-pair production.

\subsubsection{Future design work}

The beam delivery systems for each of 2 IRs at the ILC will be updated in the coming year. The same is true for the extraction lines from the collider IPs to the beam dumps. Design choices for the crossing angles of the IRs and linacs will impact polarimeter designs, as will other design choices such as the optics and locations for the upstream energy collimation. Polarimeter design studies need to be an integral part of the beam optics design studies.

One wants to find optics solutions where the beam trajectories are parallel at the upstream and downstream polarimeter Compton IPs and the collider IP. For the upstream polarimeter we need adequate energy collimation downstream of the Compton IP to mitigate against backgrounds from off-energy Compton-scattered electrons. For the downstream polarimeter, we need to evaluate its feasibility and expected performance for different IR crossing angle geometries. Extraction line optics for a downstream polarimeter should also accommodate an energy spectrometer.

The designs of the laser systems of the Compton polarimeters will be re-evaluated for optimizing the pulse energy, pulse length and pulse repetition rate, while achieving a simple and robust laser system. This will include revisiting design criteria for the measurement time needed to achieve $0.1 \%$ statistical precision and the time scale for monitoring dependence of polarization along the 1-ms train. It is also planned to evaluate a recent proposal to implement a Fabry-Perot cavity for the laser beam around the Compton IP [238]. A commercial laser with $\approx 10 \mathrm{~nJ} /$ pulse and 3-5 MHz pulse frequency could 


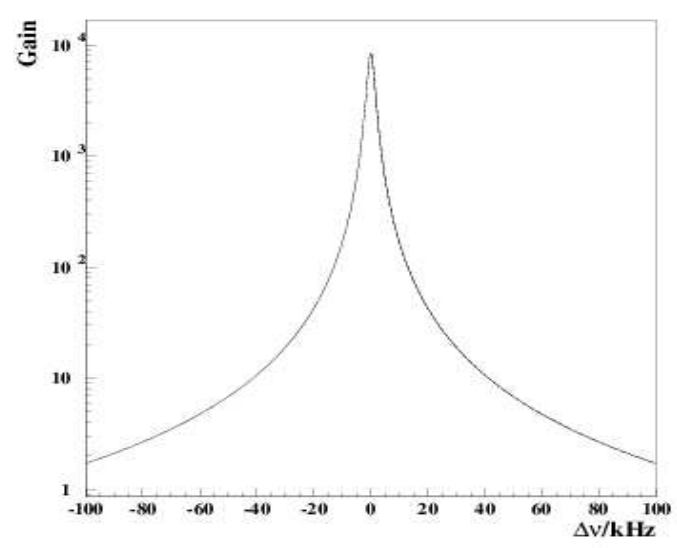

Figure 5.19: Gain of a $2 \mathrm{~m}$ long cavity as a function of the difference between the laser and cavity resonance frequencies. the values of the cavity mirror reflection and transmission coefficients are those of the HERA cavity [238].

be used together with a high finesse cavity to reach in excess of $\approx 1 \mathrm{~J} /$ pulse inside the cavity. In order to amplify laser beam power with a Fabry-Perot cavity one must keep such a cavity at resonance, i.e. match the cavity length and laser beam frequency very precisely. The cavity gain factor is shown in Figure 5.19. A fast feedback system would be required to ensure these conditions. Such a device is used at the CEBAF accelerator [239] and at HERA.

\subsection{Polarization measurements with collider data}

Polarization measurements with polarimeters are limited to a total precision around $0.25 \%$. In addition polarimeters measure either the polarization of the incoming beam that has not been depolarized by the beam-beam interaction or the one of the outgoing beam which has been depolarized three to four time as much as the interacting particles. On the other hand there are several processes at a linear collider whose polarization structure is known and which might be used to measure the polarization directly from data. The large luminosity of the linear collider offers the possibility to reach a precision much better then the polarimeters.

One example is the $\sin ^{2} \theta_{\text {eff }}$ measurement with the Blondel scheme at GigaZ (see sec. 2.4) where the relevant observables can be extracted directly from the data without the use of polarimeters. One has, however, to take into account that all methods using annihilation data involve some physics assumptions that have to be considered in the framework of the model in which the data are analysed. The data driven methods also cannot replace completely the polarimeters. The data methods need a large luminosity to get to a precise result while polarimeters are completely systematics limited and statistics is no problem. In any case polarimeters are thus needed for a fast machine tuning. In addition there are some assumptions in the data-methods that have to be verified or corrected with the polarimeters. In all cases the data methods need the assumption that the absolute values of the polarizations of the left and right handed states are the same. If electron and positron polarization is available the effective polarizations, explained in sec. 1.2.3 and the formulae to obtain the polarization involve linear and quadratic terms of the polarizations. For these reasons any correlations between the two beam polarizations need to be known 
from beam-beam simulations and polarimeters.

\subsubsection{Measurements with electron polarization only}

If only electron polarization is available not only the Lorenz structure of the used process is needed but the exact helicity structure needs to be known. The only process fulfilling this requirement is the V-A structure of the $W$-fermion couplings. This coupling can be utilised in two processes at a linear collider, single $W$-production and $W$-pair production. As can be seen from figure 5.20 both processes have a cross section of several pb so that a few million events are expected.

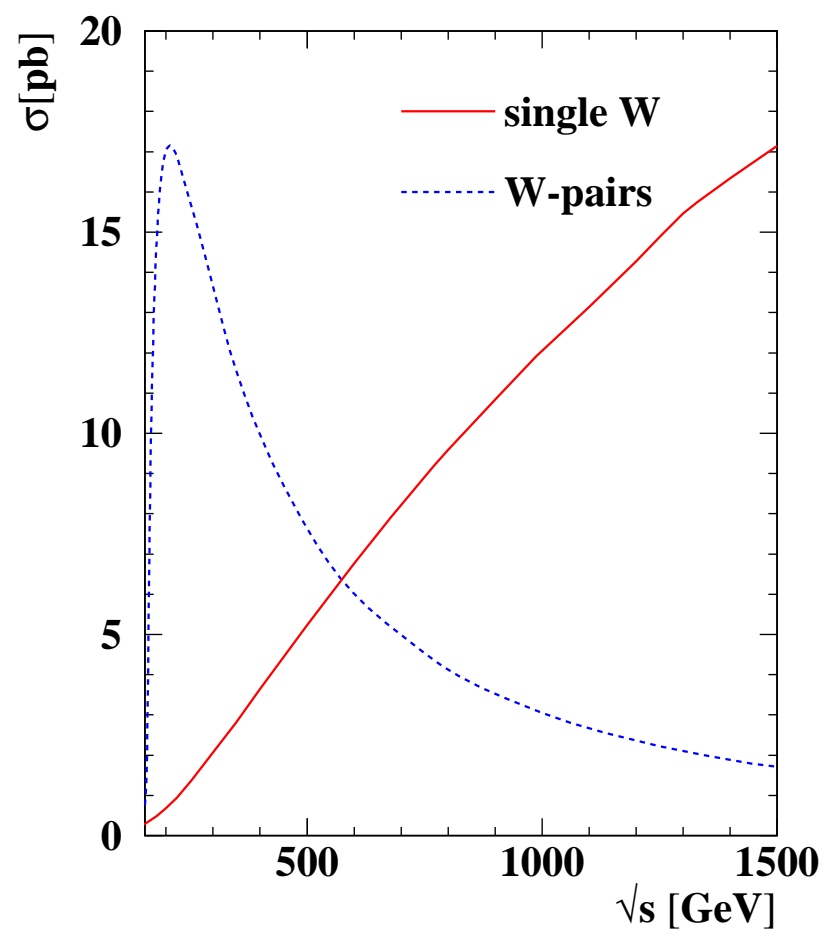

Figure 5.20: Cross section for single $W$ and $W$-pair production at a LC

$W$-pair production proceeds through the Feynman diagrams shown in fig. 2.5. In general the process is a complicated mixture of the neutrino t-channel exchange, only determined by the $W$-fermion couplings, and the $\gamma$ and $\mathrm{Z}$ s-channel exchange that also involve (anomalous) gauge couplings. However, as shown in fig. 5.21, the forward pole is completely determined by the neutrino exchange and insensitive to the anomalous couplings. For this reason it is possible to extract the polarization and the triple gauge couplings [240] simultaneously from the $W$-pair data sample. The expected precision is $\Delta P_{e^{-}} / P_{e^{-}}=0.1 \%$ for a luminosity of $500 \mathrm{fb}^{-1}$ at $\sqrt{s}=340 \mathrm{GeV}$. The correlation with the anomalous gauge couplings is negligible and the only assumption involved is that no right-handed $W$-fermion couplings appear. Experimental details of the analysis can be found in [241].

If electron and positron polarization are available, both can be measured simultaneously from the $W$-pair sample. With equal luminosity at all four helicity combinations and $P_{e^{-}}=80 \%, P_{e^{+}}=60 \%$ one gets $\Delta P_{e^{-}} / P_{e^{-}} \approx 0.1 \%$ and $\Delta P_{e^{+}} / P_{e^{+}} \approx 0.2 \%$ and negligible correlations between the polarizations and between the polarization and the cou- 


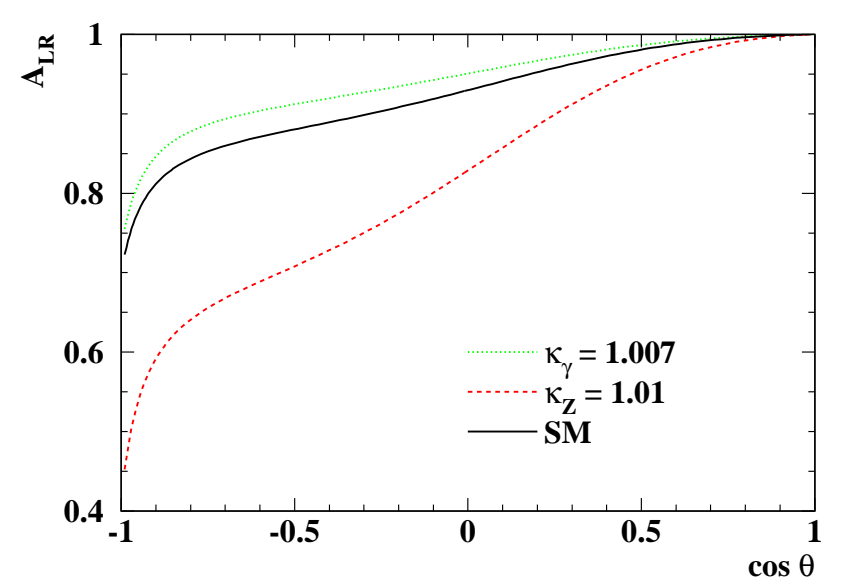

Figure 5.21: Left-right asymmetry of $W$-pair production as a function of the polar angle in the Standard Model and with anomalous triple-gauge couplings.

plings. If only $10 \%$ of the luminosity is spent on the equal helicities the polarization errors increase by roughly a factor two with $-50 \%$ correlation.

Single $W$ production is dominated by the Feynman diagram shown in fig. 2.6. Since this process involves the V-A coupling of the $W$ to fermions a $W^{-}$can only be produced from a left-handed electron and a $W^{+}$from a right-handed positron. Measuring the $W$ charge, the polarization can thus be measured for electrons and positrons separately. The outgoing electron usually disappears in the beampipe so that the $W$ charge has to be reconstructed from the $W$-decay products. This means that only leptonic $W$-decays can be used for the analysis for which no detailed simulation study exists yet. The experimental signature is a single lepton in the detector which can be measured with high efficiency and small background. Because of the usually small $W$ energy also the interference with $W$-pair production should be very small. Assuming $\sqrt{s}=500 \mathrm{GeV}, \mathcal{L}=1 \mathrm{ab}^{-1}$ and $100 \%$ efficiency for $W^{-} \rightarrow e^{-}, \mu^{-}$an error of $\Delta P_{e^{-}} / P_{e^{-}} \sim 0.15 \%$ is expected.

\subsubsection{The Blondel scheme}

If a process $e^{+} e^{-} \rightarrow \bar{f} f$ is mediated by pure s-channel vector-particle exchange the cross section for the different polarization states with electron and positron polarization available can be written as

$$
\sigma=\sigma_{u}\left[1-P_{e^{+}} P_{e^{-}}+A_{\mathrm{LR}}\left(P_{e^{+}}-P_{e^{-}}\right)\right]
$$

where $P_{e^{+}}$and $P_{e^{-}}$are the longitudinal polarizations of the positrons and electrons measured in the direction of the particle's velocity, $\sigma_{u}$ denotes the unpolarized cross section and $A_{\mathrm{LR}}$ the left-right asymmetry. If the signs of the two polarizations can be switched independently four cross sections can be measured to determine the four unknowns. From these cross sections the polarizations can be obtained, if $A_{\mathrm{LR}} \neq 0$ :

$$
P_{e^{ \pm}}=\sqrt{\frac{\left(\sigma_{+-}+\sigma_{-+}-\sigma_{++}-\sigma_{--}\right)\left(\mp \sigma_{-+} \pm \sigma_{+-}-\sigma_{++}+\sigma_{--}\right)}{\left(\sigma_{-+}+\sigma_{+-}+\sigma_{++}+\sigma_{--}\right)\left(\mp \sigma_{-+} \pm \sigma_{+-}+\sigma_{++}-\sigma_{--}\right)}}
$$

where in $\sigma_{i j} i$ denotes the sign of the positron and $j$ the sign of the electron polarization. A drawback of this method is that some luminosity needs to be spent with the same helicities for both beams which is not very interesting for most physics processes. 
To measure the polarization with this scheme two processes have been considered,

- $\mathrm{e}^{+} \mathrm{e}^{-} \rightarrow \bar{f} f$ with $\sqrt{s^{\prime}} \approx \sqrt{s}$;

- radiative return events $\left(\mathrm{e}^{+} \mathrm{e}^{-} \rightarrow \mathrm{Z} \gamma \rightarrow \overline{\mathrm{f}} \mathrm{f} \gamma\right.$ ).

The cross section and left right asymmetry for the two processes at $\sqrt{s}=350$ and $500 \mathrm{GeV}$ are given in table 5.5. Both cross sections scale approximately with $1 / s$. The high-energy

\begin{tabular}{|c|c|c|c|c|}
\hline$\sqrt{s}$ & $\sigma_{R R}$ & $A_{\mathrm{LR}}(R R)$ & $\sigma_{H E}$ & $A_{\mathrm{LR}}(H E)$ \\
\hline $340 \mathrm{GeV}$ & $17 \mathrm{pb}$ & 0.19 & $5 \mathrm{pb}$ & 0.50 \\
$500 \mathrm{GeV}$ & $7 \mathrm{pb}$ & 0.19 & $2 \mathrm{pb}$ & 0.50 \\
\hline
\end{tabular}

Table 5.5: Cross section and asymmetry for high-energy and radiative-return $\bar{f} f$ events.

events can be measured with high efficiency and almost no background. However the analysis relies on the assumption of s-channel vector-exchange, so for analyses like the search for R-parity-violating sneutrinos the results cannot be used.

On the contrary radiative return events contain on-shell Z-decays which are well understood from LEP'1 and SLD. In about $90 \%$ of the events the high-energy photon is lost in the beampipe. These events can be reconstructed kinematically and most backgrounds can be rejected. However, at ILC energies the cross section for the fusion process $\mathrm{e}^{+} \mathrm{e}^{-} \rightarrow \mathrm{Ze}^{+} \mathrm{e}^{-}$is of the same order as the signal. In those events one electron has almost the beam energy and stays at low angle while the other is extremely soft and also often lost in the beampipe resulting in a $\sim 30 \%$ background of Zee events in the radiative return sample. The only way Zee events can be rejected is to require a photon above $7^{\circ}$ where photons and electrons can be separated by the tracking detectors. Applying some additional event selection cuts on the hadronic mass and the balance of the event, about $9 \%$ of the radiative return events are accepted with only a small Zee background. However in these events the slow electron is seen in the detector, so that they can easily be rejected by vetoing on an isolated electron.

Assuming $\left|P_{e^{-}}\right|=80 \%,\left|P_{e^{+}}\right|=60 \%$, an integrated luminosity of $500 \mathrm{fb}^{-1}$ at $\sqrt{s}=$ $340 \mathrm{GeV}$ and $50 \%$ or $10 \%$ of the luminosity spent with both beam polarizations with the same sign table 5.6 shows the obtainable errors on the two polarizations and their correlation. Due to the scaling of the cross sections the errors are about a factor $\sqrt{2}$ larger at $500 \mathrm{GeV}$. It should be noted that the relative errors scale approximately with the product of the polarizations.

\begin{tabular}{|c|r|r|c|r|r|r|}
\hline & \multicolumn{3}{|c|}{$\mathcal{L}_{ \pm \pm} / \mathcal{L}=0.5$} & \multicolumn{3}{|c|}{$\mathcal{L}_{ \pm \pm} / \mathcal{L}=0.1$} \\
& HE & \multicolumn{1}{|c|}{ rr } & $W W$ & \multicolumn{1}{c|}{ HE } & \multicolumn{1}{c|}{ rr } & \multicolumn{1}{|c|}{$W W$} \\
\hline$\Delta P_{e^{-}} / P_{e^{-}}[\%]$ & 0.10 & 0.51 & 0.07 & 0.21 & 1.11 & 0.11 \\
$\Delta P_{e^{+}} / P_{e^{+}}[\%]$ & 0.12 & 0.53 & 0.11 & 0.15 & 1.13 & 0.21 \\
corr & -0.49 & -0.91 & 0 & -0.56 & -0.93 & -0.52 \\
\hline
\end{tabular}

Table 5.6: Relative polarization error using the Blondel scheme for $\sqrt{s}=340 \mathrm{GeV}, \mathcal{L}=$ $500 \mathrm{fb}^{-1},\left|\mathrm{P}_{\mathrm{e}^{-}}\right|=80 \%,\left|\mathrm{P}_{\mathrm{e}^{+}}\right|=60 \%$ (HE = High energy events, $\mathrm{rr}=$ radiative return, $W W$ $=W$-pair production). 
Radiative corrections to the form of eq. (5.11) have been checked with the KK Monte Carlo [242]. For the high-energy events and for the radiative-return events with a seen photon they are negligible. For the radiative return events where the photon is lost in the beampipe, which are not used in this analysis, the corrections are at the percent level.

Because of the high losses in the selection of the radiative-return events, the errors in the single polarizations seem rather large. However the large negative correlation reduces the error substantially for the effective polarizations needed in the analysis. Table 5.7 compares the errors on the effective polarizations for the setups shown in table 5.6 and for polarimeter measurements assuming 0 or $50 \%$ correlation between the two polarimeters.

The effective polarizations considered are:

- $P_{\text {eff }}=\frac{\left|P_{e^{-}}\right|+\left|P_{e^{+}}\right|}{1+\left|P_{e^{-}} P_{e^{+}}\right|}$, relevant for $A_{\mathrm{LR}}$ with s-channel vector exchange;

- $\left|P_{e^{-}} P_{e^{+}}\right|$, relevant for the cross section suppression/enhancement with s-channel vector exchange;

- $\left|P_{e^{-}}\right|+\left|P_{e^{+}}\right|-\left|P_{e^{-}} P_{e^{+}}\right|$, relevant for the cross section suppression/enhancement for t-channel $W$-pair production.

Due to the high anti-correlation, even the results from the radiative-return analysis with one tenth of the luminosity at the low cross sections are competitive with Compton polarimetry.

\begin{tabular}{|c|c|c|c|c|c|c|c|c|c|}
\hline & value & \multicolumn{9}{|c|}{ Rel. error [\%] } \\
\cline { 3 - 10 } & & \multicolumn{4}{|c|}{$\mathcal{L}_{ \pm \pm} / \mathcal{L}=0.5$} & \multicolumn{2}{c|}{$\mathcal{L}_{ \pm \pm} / \mathcal{L}=0.1$} & \multicolumn{2}{c|}{ Polarimeter } \\
& & HE & rr & $W W$ & HE & rr & $W W$ & $\rho=0$ & $\rho=0.5$ \\
\hline$\left(\left|P_{e^{-}}\right|+\left|P_{e^{+}}\right|\right) /\left(1+\left|P_{e^{-}} P_{e^{+}}\right|\right)$ & 0.95 & 0.02 & 0.08 & 0.02 & 0.05 & 0.17 & 0.02 & 0.13 & 0.16 \\
$\mid P_{e^{-}} P_{e^{+}}$ & 0.48 & 0.11 & 0.22 & 0.13 & 0.18 & 0.42 & 0.18 & 0.71 & 0.87 \\
$\left|P_{e^{-}}\right|+\left|P_{e^{+}}\right|-\left|P_{e^{-}} P_{e^{+}}\right|$ & 0.92 & 0.03 & 0.12 & 0.03 & 0.06 & 0.25 & 0.03 & 0.19 & 0.21 \\
\hline
\end{tabular}

Table 5.7: Relative error on the effective polarizations for the discussed setups and $\sqrt{s}=$ $340 \mathrm{GeV}, \mathcal{L}=500 \mathrm{fb}^{-1},\left|\mathrm{P}_{\mathrm{e}^{-}}\right|=80 \%,\left|\mathrm{P}_{\mathrm{e}^{+}}\right|=60 \%$. For the polarimeter a total error of $0.5 \%$ has been assumed. ( $\mathrm{HE}=$ High energy events, $\mathrm{rr}=$ radiative return, $W W=W$-pair production).

Additional information can be obtained from Bhabha scattering [243]. Elastic electronelectron (Møller) and positron-electron (Bhabha) scattering processes have large and wellknown [244-247] spin asymmetries which can be exploited for polarimetry. However, in contrast to weak interaction processes (such as single-W production) it is necessary that both colliding particles are polarized. If both beams in the collider are polarized, it is therefore possible to determine the product of the beam polarizations relatively quickly from the copious Bhabha events observed in the principal collider detector, even without dedicated beam polarimeters. In this context it is worthwhile to mention that the spin asymmetries for Bhabha scattering reach their maximum value of $7 / 9^{*}$ for symmet-

*The maximum value of the Møller and Bhabha asymmetry is $7 / 9$ for the case of longitudinal beam polarizations, which is the case of principal interest. For transverse beam polarization, the maximum value is only $\pm 1 / 9$ for parallel beam spins, with the sign depending on the azimuth angle between the spin and the scattering plane. The asymmetry vanishes for transverse beam polarizations with orthogonal spin directions. These properties are useful for transverse spin experiments, cf. section 5.5.5. 
ric pairs at $\theta_{c m}=90^{\circ}$ which guarantees excellent coverage in the collider detector.

A detailed study including Z-exchange remains to be done, however the pure QED term constraints the two polarisations along the small axis of the error ellipse from the Blondel scheme, so that the final gain is probably not very large.

\subsubsection{Experimental aspects}

Although the methods presented here measure the luminosity-weighted polarization directly from the annihilation data, some experimental assumptions are involved. In all cases it is assumed that the absolute values of the polarization of the left- and righthanded state are the same and possible corrections have to be obtained from polarimeters. If the polarization is written as $P= \pm\langle|P|\rangle+\delta P$ The shift in the measured polarization using $W \mathrm{~s}$ in the case of electron polarization only is given by $\Delta P / P=\delta P$

Using the Blondel scheme with polarized electrons and positrons, the corresponding errors are

$$
\begin{aligned}
\Delta P_{e^{-}}= & 1.0 \delta P_{e^{-}}+0.6 \delta P_{e^{+}} \\
\Delta P_{e^{+}}= & -0.5 \delta P_{e^{-}}-0.7 \delta P_{e^{+}}
\end{aligned}
$$

for the high-energy events and

$$
\begin{aligned}
& \Delta P_{e^{-}}=2.4 \delta P_{e^{-}}+2.1 \delta P_{e^{+}} \\
& \Delta P_{e^{+}}=-1.7 \delta P_{e^{-}}-1.7 \delta P_{e^{+}}
\end{aligned}
$$

for the radiative-return sample.

The corresponding corrections have to be obtained from polarimeters. This is possible in a Compton polarimeter where the laser polarization can be flipped easily. To assure that the electron-laser luminosity does not depend on the laser polarization, or to correct for such effects, one should have a multichannel polarimeter with a large lever arm in the analysing power.

If electron and positron polarization is available, in the formulae for the effective polarizations and for the Blondel scheme products of the two polarizations appear so that one has to understand the correlations between the electron and positron polarization. In principle there can be a correlation due to the depolarization in the bunch. Studies with CAIN [248], however, indicate that these correlations are small. Another source of correlation can come from time dependences or spatial correlations due to the beam delivery system. If half of the luminosity is taken with a polarization $5 \%$ higher and the other half $5 \%$ lower than average, the polarizations obtained with the Blondel scheme are off by around $0.25 \%$, affecting the effective polarization by the same amount. Measuring the polarization with polarimeters would only result in a $0.16 \%$ error in the effective polarization.

Time correlations have to be tracked with polarimeters. Spatial correlations due to the beam-delivery system have to be obtained from simulations and should be minimised already in the design. 


\section{Chapter 6}

\section{Concluding Remarks}

Strong theoretical arguments and perhaps some experimental evidence indicate that the Standard Model of elementary particles is not the final theory of everything, and a variety of possible extensions have been proposed. Many of these envisage new building blocks and new interactions near the Fermi scale, and perhaps at higher mass scales. The choice among such scenarios, and hence the direction of new physics beyond the Standard Model, is of paramount importance. As the first machine to probe directly the $\mathrm{TeV}$ scale, the LHC will surely provide new discoveries and valuable information in this regard. However, it is generally agreed that the clean and precise environment of $e^{+} e^{-}$ collisions at the ILC is ideally suited to the search for new physics and for determining precisely the underlying structure of the new interactions, whatever direction the LHC results may favour.

As we have demonstrated here, polarized beams will be very powerful tools to help reach these goals. The physics examples presented here have shown that having both beams polarized simultaneously will provide high flexibility and a very efficient means for the disentanglement of non-standard effects in various new physics scenarios, and their positive identification, as well as having the capacity to observe surprises in precision tests of the Standard Model. Having two polarized beams available is crucial for determining the properties and the quantum numbers of new particles, and to test fundamental model assumptions, as we have shown in the specific example of supersymmetry.

The larger number of observables accessible with two polarized beams provide better tools for revealing the structure of the underlying physics and determining new physics parameters in model-independent analyses. New signals may become accessible by maximizing the analyzing power using suitable beam polarizations combined with high luminosity. In many cases, as we have shown, double beam polarization enables better statistics to be obtained and the dominant systematical errors in indirect searches to be reduced. This can give access to physics scales that may be far beyond the direct kinematical reach of accelerators.

To exploit fully high-precision tests of the Standard Model at GigaZ, both beams must be polarized. To make full use of the extremely high statistics, the beam polarizations must be known with high precision, which cannot be provided by conventional polarimetry methods. However, the required precision can be achieved in the Blondel scheme, where both electron and positron polarizations are needed.

Further, with both beams polarized, one has the possibility to exploit transverselypolarized beams for physics studies. This option provides new and efficient observables 
for the detection of possible sources of CP violation. Additionally, it becomes easier to observe the effects of massive gravitons and to distinguish between different models with extra spatial dimensions, far below the threshold for the spin-2 excitations. One can test specific triple-gauge-boson couplings which are not accessible otherwise. The power to identify new physics with polarized beams would represent a step forward of the utmost importance for our understanding of fundamental interactions.

An overview of the physics benefits coming from positron polarization has been given in this report, also in comparison with the case of only polarized electrons. An overview of the machine design for polarized beams at the ILC has also been given. SLAC experiments established that reliable high-energy electron beams with a polarization of $85-90 \%$ can be provided for an LC. The standard source for polarized electrons is a DC gun with a strained superlattice photocathode. To obtain polarized positrons at an LC, two methods are discussed:

a) Circularly-polarized photons from a helical undulator can be used to generate longitudinally-polarized positrons in a target via $e^{ \pm}$pair production;

b) Circularly-polarized photons can also be obtained by backscattering of laser light off the electron beam.

A demonstration experiment for an undulator-based polarized positron source, E-166 at SLAC, is currently running. Prototypes of both superconducting and permanent magnets for helical undulators for the ILC with lengths of 10 or 20 periods are already under construction at the Daresbury and Rutherford Laboratories. Meanwhile, the concept of the laser-based positron source is being tested in an experiment at KEK.

As already emphasized, precise physics analyses at an LC require accurate beam polarization measurements. The primary polarimetry measurement at the ILC will be performed with a Compton polarimeter, with an expected accuracy $\Delta P_{e^{-}} / P_{e^{-}}=0.25 \%$. The polarimeters can be located upstream or downstream of the Interaction Point (IP), and it would be desirable to implement polarimeters at both locations. A downstream polarimeter may require a large crossing angle at the IP.

Since polarimeters measure either the polarization of the incoming beam before it has been depolarized by the beam-beam interaction, or that of the outgoing beam after depolarization in the interaction region, it is desirable to measure the polarization also directly from the data via processes with precisely known polarization structures, such as $W W$ production. Alternatively, in the GigaZ option, one may apply the Blondel scheme, for which polarized positrons are needed. Methods for measuring the polarization using annihilation data involve physics assumptions that have to be considered in the framework of the model in which the data are analysed. The data-driven methods therefore cannot replace completely the polarimeters but provide an independent and complementary measurement. The data methods offer the possibility to reach a precision even better than the polarimeters, if the underlying physics is well understood.

In summary, it has been demonstrated that having simultaneously polarized $e^{-}$and $e^{+}$ beams is a very effective tool for direct as well as indirect searches for new physics. This option provides ideal preparation even for unexpected new physics. Polarized positrons are necessary for several specific physics issues, and enrich the physics potential considerably. Techniques and engineering design for a polarized positron source are becoming well advanced. Therefore, including a polarized positron source in the ILC baseline design can be considered. 


\section{Appendix A}

\section{Tools for simulation studies: MC event generators}

The use of numerical programs based on Monte Carlo (MC) techniques has become essential in performing any detailed experimental analysis in collider physics. In this Appendix the so-called event generators with inclusion of beam polarizations are briefly described. These programs must be interfaced to both detector simulations and beam energy spectra to give a complete picture of the actual physical process.

Most MC event generators fall into one of two classes:

a) general-purpose (or multi-purpose) event generators

which aim to perform the full simulation of the event starting with the initial-state collider beams, proceeding through the parton-shower (wherein coloured particles are perturbatively evolved from the hard scale of the collision to an infrared cut-off) and hadronization (wherein partons left after the said perturbative evolution are formed into the observed mesons and baryons) stages;

b) parton-level event generators

which typically perform the hard scattering part of the simulation only, perhaps including decays, and rely on one of the general-purpose generators for the rest of the simulation.

During the LEP-era the experiments relied on the general-purpose event generators for the description of hadronic final states together with more accurate parton-level programs interfaced to the former ones for specific processes, e.g., two- and four-fermion production. At a future linear collider, as one wishes to study final states with higher multiplicities, e. g. six or even eight particles, this combined approach will become more important as these final states cannot be described by the general-purpose event generators.

\section{General-purpose event generators}

General-purpose event generators include HERWIG [249], ISAJET [250] and PYTHIA [251], which use different phenomenological models and approximations. The major differences between these programs are in the approximations used in the parton shower evolution and the hadronisation stage. There are also major differences between the generators in the treatment of spin correlation and polarization effects. 
Both ISAJET and HERWIG include longitudinal polarization effects in both SM and supersymmetric (SUSY) production processes, while PYTHIA includes both longitudinal and transverse polarizations in many processes.

Another important difference is in the treatment of the subsequent decay of any heavy particle produced in the hard process. While HERWIG includes the full correlations in any subsequent decays using the method described in [252], both ISAJET and PYTHIA only include these effects in some processes, such as $W$-pair production. Including transverse polarization also in the HERWIG production stage is certainly possible.

The only program currently available in $\mathrm{C}++$ which is capable of generating physics results is SHERPA (based on the APACIC++ [253] parton shower). Work is however underway to rewrite both PYTHIA [254] and HERWIG [255] in C++. Given the new design and structure of these programs, the treatment of both spin correlation and polarization effects should be much better than in the current FORTRAN programs. HERWIG++ should include full polarization and correlation effects using the method of [252].

\section{Parton-level event generators}

There are a large number of programs available which calculate some set of hard processes, and are interfaced to one of the general-purpose generators, most often PYTHIA. Many of the two- [256] and four-fermion [257] generators were used by the LEP collaborations (see report of the LEP-II MC workshop for their detailed discussion). Some programs are written for six-fermion processes, e.g., ref. LUSIFER [258], SIXFAP [259], EETT6F [260] and SIXPHACT [261]. These generators usually include state-of-the-arts calculations of the generated processes. Many of these codes use helicity-amplitude techniques to calculate the matrix elements and therefore either already include polarization effects or could easily be modified to do so.

There are a number of codes available, which are capable of calculating and integrating the matrix elements for large numbers of final-state particles automatically:

- AMEGIC++ [262] makes use of helicity amplitudes and is part of SHERPA.

- COMPHEP [263] uses the traditional trace techniques to evaluate the matrix elements; therefore it is at present not suitable to investigate polarization/spin effects. However, the conversion to the use of helicity amplitudes techniques is currently planned.

- GRACE [264] (with the packages BASES and SPRING) uses the calculation of matrix elements via helicity amplitude techniques.

- HELAC/P HEGAS calculates cross sections [265,266].

- MADGRAP H MADEVENT [267] uses helicity amplitude techniques for the matrix elements to calculate cross sections (based on the HELAS [268] subroutines).

- WHIZARD [269] is an integration package which can use either COMP HEP, MADGRAPH or $\mathrm{O}^{\prime} \mathrm{MEGA}{ }^{*}$ [271] to calculate the matrix elements. Transverse beam polarization is included.

The implementation of polarization and correlation effects differs between these programs. In general, apart from COMPHEP (as noted), these programs are all based on helicity amplitude techniques at some point in the calculation and therefore the inclusion of both transverse and longitudinal beam polarization is possible even where it is not currently implemented.

${ }^{*} \mathrm{O}^{\prime} \mathrm{MEGA}$ uses the approach of [270] to evaluate the matrix elements but does not yet include any QCD processes. 


\section{Event generators for supersymmetry}

Polarization and spin correlation effects are particularly important in studying SUSY scenarios, in order to measure the fundamental parameters of the underlying model.

HERWIG, PYTHIA and ISAJET all include longitudinal polarization effects in SUSY production processes. There is also a parton-level program SUSYGEN [272], interfaced to PYTHIA, which includes these effects.

These programs also differ in the inclusion of the correlations in the subsequent decays of the particles. While SUSYGEN includes these correlations using helicity amplitude techniques and HERWIG uses the method of [252], these effects are generally not included in either PYTHIA or ISAJET.

Among the parton-level programs, at present only COMP HEP and GRACE and AMEGIC++ include SUSY processes, limitedly to the MSSM (although extensions to non-minimal SUSY models are now planned within COMP HEP). WHI ZARD also supports the MSSM, optionally with anomalous couplings, but model extensions or completely different models can be added. Finally, MADGRAPH can be extended to add the additional SUSY interactions that are needed, as (most of) the prototype subroutines for the latter already exist in the HELAS library. 


\section{Acknowledgements}

The work was partially supported by the Commission of the European Communities under the 6th Framework Programme 'Structuring the European Research Area', contract number RIDS-011899. This work is also supported by the 'Fonds zur Förderung der wissenschaftlichen Forschung' (FWF) of Austria, projects No. P16592-N02 and by the European Community's Human Potential Programme under contract HPRN-CT-200000149, by the 'Deutsche Forschungsgemeinschaft' (DFG) under contract Fr 1064/5-1, by the Polish State Committee for Scientific Research Grant No 2 P03B 04024 and by the DOE project with the Contract No. DE-AC02-76SF00515. S.Hes. is supported by Göran Gustafsson Foundation. GMP would like to thank F. Richard, D. Schulte, T. Teubner, P. Uwer and N. Walker for many helpful discussions. 


\section{Bibliography}

[1] J. A. Aguilar-Saavedra et al. [ECFA/DESY LC Physics Working Group], eds. R.D. Heuer, D. Miller, F. Richard and P. Zerwas, arXiv:hep-ph/0106315; F. Richard, J. R. Schneider, D. Trines and A. Wagner, arXiv:hep-ph/0106314.

[2] T. Abe et al. [American Linear Collider Working Group Collaboration], in Proc. of the APS/DPF/DPB Summer Study on the Future of Particle Physics (Snowmass 2001) ed. N. Graf, SLAC-R-570 Resource book for Snowmass 2001, 30 Jun - 21 Jul 2001, Snowmass, Colorado; hep-ex/0106055, hep-ex/0106056, hep-ex/0106057, hep-ex/0106058; $\mathrm{K}$. Abe et al. [ACFA Linear Collider Working Group Collaboration], arXiv:hepph/0109166; K. Abe et al., KEK-REPORT-2003-7

[3] ICFA, Parameters for the Linear Collider, see webpage: www.interactions.org/linearcollider/documents/index.htm.

[4] Ch. Baltay, see talk given at the ECFA/DESY LC-workshop, Obernai, October 1999; http:/ / www.desy.de/conferences/ecfa-desy-lc98.html.

[5] G. Moortgat-Pick and H. M. Steiner, Eur. Phys. J. directC 3 (2001) 6 [arXiv:hep$\mathrm{ph} / 0106155]$.

[6] E. Accomando et al., arXiv:hep-ph/0412251.

[7] H. E. Haber, arXiv:hep-ph/9405376.

[8] F. M. Renard, Basics of Electron Positron Collisions, Editions Frontieres, Dreux 1981. F. M. Renard, Z. Phys. C 45 (1989) 75; M. Jacob and G. C. Wick, Annals Phys. 7 (1959) 404 [Annals Phys. 281 (2000) 774].

[9] K. I. Hikasa, Phys. Rev. D 33 (1986) 3203;

[10] A. Blondel, CERN-PPE-93-125; L. Knudsen et al., Phys. Lett. B 270 (1991) 97; A. Djouadi, F. M. Renard and C. Verzegnassi, Phys. Lett. B 241 (1990) 260;

[11] A. A. Sokolov and I. M. Ternov, Phys. Dokl. 8 (1964) 1203.

[12] G. V. Dass and G. G. Ross, Phys. Lett. B 57 (1975) 173; G. V. Dass and G. G. Ross, Nucl. Phys. B 118 (1977) 284.

[13] R. Budny, Phys. Rev. D 14 (1976) 2969; C. P. Burgess and J. A. Robinson, Int. J. Mod. Phys. A 6 (1991) 2707; for a recent discussion, see K. Desch, talk given at the International Workshop on the Linear Collider, LCWS2002, Jeju Island, Korea, Aug. 2002. 
[14] T. Omori, KEK-PREPRINT-98-237 Presented at the 1st ACFA Workshop on Physics Detector at the Linear Collider, Beijing, China, 26-28 Nov 1998

[15] K. Flottmann, DESY-95-064; K. Fujii and T. Omori, KEK-PREPRINT-95-127.

[16] P. C. Rowson, D. Su and S. Willocq, Ann. Rev. Nucl. Part. Sci. 51 (2001) 345 [arXiv:hep-ph/0110168].

[17] K. Abe et al. [SLD Collaboration], Phys. Rev. Lett. 84 (2000) 5945 [arXiv:hepex/0004026].

[18] S.B. Gunst, L.A. Page, Phys. Rev. 92 (1953) 970.

[19] M. Woods [SLD Collaboration], arXiv:hep-ex/9611005.

[20] S. Heinemeyer, S. Kraml, W. Porod and G. Weiglein, JHEP 0309 (2003) 075 [arXiv:hepph/0306181]; G. Weiglein, Nature 429 (2004) 613.

[21] D. Atwood and A. Soni, Phys. Rev. D 45 (1992) 2405;

[22] W. Hollik, J. I. Illana, S. Rigolin, C. Schappacher and D. Stockinger, Nucl. Phys. B 551 (1999) 3 [Erratum-ibid. B 557 (1999) 407] [arXiv:hep-ph/9812298].

[23] J. H. Kuhn, LC-TH-2001-004; M. Jezabek, Nucl. Phys. Proc. Suppl. 51C (1996) 60; R. Harlander, M. Jezabek, J. H. Kuhn and M. Peter, Z. Phys. C 73 (1997) 477 [arXiv:hep-ph/9604328].

[24] B. Grzadkowski and Z. Hioki, Nucl. Phys. B 585 (2000) 3 [arXiv:hep-ph/0004223].

[25] S. D. Rindani, Pramana 61 (2003) 33 [arXiv:hep-ph/0304046].

[26] U. Baur, A. Juste, L. H. Orr and D. Rainwater, Phys. Rev. D 71 (2005) 054013 [arXiv:hep-ph/0412021].

[27] J. A. Aguilar-Saavedra and T. Riemann, arXiv:hep-ph/0102197.

[28] E. W. N. Glover et al., Acta Phys. Polon. B 35 (2004) 2671 [arXiv:hep-ph/0410110]; J. A. Aguilar-Saavedra, Acta Phys. Polon. B 35 (2004) 2695 [arXiv:hep-ph/0409342].

[29] L. Chikovani and T. Djobava, arXiv:hep-ex/0205016; M. Beneke et al., arXiv:hep$\mathrm{ph} / 0003033$.

[30] P. M. Zerwas ed., "e+ e- collisions at 500-GeV: The physics potential. Proceedings, Workshops, DESY Reports 92-123 A+B, 93-123 C, 96-123 D, 99-123 F.

[31] K. Desch, September 1999 (rates are calculated with program PYTHIA); see also talk by K. Desch, ECFA/DESY LC-workshop, Obernai, October 1999, webpage: http://ireswww.in2p3.fr/ires/ecfadesy/; D. J. Summers, Phys. Lett. B 274 (1992) 209.

[32] A. Gurtu, Prepared for 30th International Conference on High-Energy Physics (ICHEP 2000), Osaka, Japan, 27 Jul - 2 Aug 2000 
[33] S. Heinemeyer, W. Hollik and G. Weiglein, Eur. Phys. J. C 9 (1999) 343 [arXiv:hepph/9812472]; G. Degrassi, S. Heinemeyer, W. Hollik, P. Slavich and G. Weiglein, Eur. Phys. J. C 28 (2003) 133 [arXiv:hep-ph/0212020].

[34] B. Kniehl, summer 2000; see also talk by B. Kniehl at ECFA/DESY LCworkshop, Hamburg, September 2000, webpage: http://www.desy.de/ ecfadesy/transparencies/.

[35] K. Hagiwara, S. Ishihara, J. Kamoshita and B. A. Kniehl, Eur. Phys. J. C 14 (2000) 457 [arXiv:hep-ph/0002043]; M. Davier, L. Duflot, F. Le Diberder and A. Rouge, Phys. Lett. B 306 (1993) 411.

[36] K. Hagiwara and M. L. Stong, Z. Phys. C 62 (1994) 99 [arXiv:hep-ph/9309248].

[37] K. Hagiwara, R. D. Peccei, D. Zeppenfeld and K. Hikasa, Nucl. Phys. B 282 (1987) 253. K.J.F. Gaemers, G.J. Gounaris, Z. Phys. C 1 (1979) 259.

[38] G. J. Gounaris and C. G. Papadopoulos, Eur. Phys. J. C 2 (1998) 365 [arXiv:hepph/9612378]. P. J. Dervan, A. Signer, W. J. Stirling and A. Werthenbach, J. Phys. G 26 (2000) 607 [arXiv:hep-ph/0002175].

[39] O. Nachtmann, F. Nagel, and M. Pospischi1, hep-ph/0404006.

[40] M. Diehl, O. Nachtmann and F. Nagel, Eur. Phys. J. C 27 (2003) 375 [arXiv:hep$\mathrm{ph} / 0209229]$.

[41] S. Schael it et al. [ALEPH Collaboration], Phys. Lett. B 614 (2005) 7; A. Heister et al. [ALEPH Collaboration], Eur. Phys. J. C 21 (2001) 423 [arXiv:hep-ex/0104034];

P. Abreu et al. [DELPHI Collaboration], Phys. Lett. B 459 (1999) 382;

M. Acciarri et al. [L3 Collaboration], Phys. Lett. B 467 (1999) 171 [arXiv:hepex/9910008];

M. Acciarri et al. [L3 Collaboration], Phys. Lett. B 487 (2000) 229 [arXiv:hepex/0007005].

[42] M. Diehl and O. Nachtmann, Z. Phys. C 62 (1994) 397.

[43] O. Nachtmann and F. Nagel, hep-ph/0407224.

[44] M. Diehl, O. Nachtmann and F. Nagel, Eur. Phys. J. C 32, 17 (2003) [hep-ph/0306247].

[45] M. Diehl and O. Nachtmann, Eur. Phys. J. C 1 (1998) 177 [arXiv:hep-ph/9702208].

[46] W. Menges, LC-PHSM-2001-022.

[47] G. Abbiendi et al. [OPAL Collaboration], Eur. Phys. J. C 19 (2001) 1 [arXiv:hepex/0009022]; G. Abbiendi et al. [OPAL Collaboration], Eur. Phys. J. C 19 (2001) 229 [arXiv:hep-ex/0009021].

[48] M. Pohl and H. J. Schreiber, DESY-99-030.

[49] J. Fleischer, K. Kolodziej and F. Jegerlehner, Phys. Rev. D 49, 2174 (1994); 
[50] S. Heinemeyer, W. Hollik, G. Weiglein, hep-ph/0412214; S. Heinemeyer and G. Weiglein, JHEP 0210 (2002) 072 [arXiv:hep-ph/0209305].

[51] R. Hawkings and K. Monig, Eur. Phys. J. directC 1 (1999) 8 [arXiv:hep-ex/9910022]; K. Mönig, LC-PHSM-1999-2-TESLA.

[52] G. Wilson, LC-PHSM-2001-009; U. Baur, R. Clare, J. Erler, S. Heinemeyer, D. Wackeroth, G. Weiglein and D. R. Wood, in Proc. of the APS/DPF/DPB Summer Study on the Future of Particle Physics (Snowmass 2001) ed. N. Graf, eConf C010630 (2001) P122 [arXiv:hep-ph/0111314].

[53] P. C. Rowson and M. Woods, Contr. to 5th international Linear Collider Workshop (LCWS 2000), Fermilab, Batavia, Illinois, 24-28 Oct 2000. Published in Batavia 2000, Physics and experiments with future linear e+e-colliders, 515-520, SLAC-PUB-8745, Dec.2000, hep-ex/0012055.

[54] A. Blondel, Phys. Lett. B202 (1988) 145.

[55] G. Alexander and E. Reinherz-Aronis, LC-PHSM-2003-032.

[56] S. Heinemeyer, T. Mannel and G. Weiglein, arXiv:hep-ph/9909538; S. Heinemeyer and G. Weiglein, arXiv:hep-ph/0012364.

[57] J. Erler, S. Heinemeyer, W. Hollik, G. Weiglein and P. M. Zerwas, Phys. Lett. B 486 (2000) 125 [arXiv:hep-ph/0005024].

[58] K. Mönig, private communication.

[59] R. Barate et al. [ALEPH Collaboration], Phys. Lett. B 565 (2003) 61 [arXiv:hepex/0306033].

[60] [LEP Higgs working group], hep-ex/0107030; hep-ex/0107031; LHWG Note 2004-1, see: lephiggs . web. cern. ch/LEPHIGGS/papers.

[61] P. S. Wells, Eur. Phys. J. C 33 (2004) S5.

[62] B. C. Allanach, A. Djouadi, J. L. Kneur, W. Porod and P. Slavich, JHEP 0409 (2004) 044 [arXiv:hep-ph/0406166].

[63] S. Heinemeyer, W. Hollik and G. Weiglein, Comput. Phys. Commun. 124 (2000) 76 [arXiv:hep-ph/9812320]; S. Heinemeyer, arXiv:hep-ph/0407244; T. Hahn, S. Heinemeyer, W. Hollik and G. Weiglein, in preparation;

The code is obtainable at www. feynhiggs . de.

[64] Contribution by S. Heinemeyer, G. Weiglein, June 2005.

[65] J. R. Ellis, S. Heinemeyer, K. A. Olive and G. Weiglein, JHEP 0502 (2005) 013 [arXiv:hep-ph/0411216].

[66] W. Bernreuther, U. Löw, J. P. Ma and O. Nachtmann, Z. Phys. C 43 (1989) 117; J. G. Körner, J. P. Ma, R. Münch, O. Nachtmann and R. Schöpf, Z. Phys. C 49 (1991) 447. 
[67] W. Bernreuther, G. W. Botz, D. Bruß, P. Haberl and O. Nachtmann, Z. Phys. C 68 (1995) 73 [arXiv:hep-ph/9412268]; P. Haberl, arXiv:hep-ph/9611430; O. Nachtmann and C. Schwanenberger, Eur. Phys. J. C 9 (1999) 565 [arXiv:hep-ph/9901343]; O. Nachtmann and C. Schwanenberger, Eur. Phys. J. C 13 (2000) 315 [arXiv:hepph/9909527].

[68] O. Nachtmann and C. Schwanenberger, Eur. Phys. J. C 32 (2004) 253 [arXiv:hep$\mathrm{ph} / 0308198]$.

[69] Contribution by C. Schwanenberger, October 2004.

[70] D. Buskulic et al. [ALEPH Collaboration], Phys. Lett. B 384 (1996) 365; D. Topaj, "Suche nach CP-Verletzung in hadronischen Z-Zerfällen", Diploma thesis (1997), University of Heidelberg (unpublished); T. Pauly, "Suche nach CP-Verletzung in den 4-Jet-Zerfällen des $Z^{0 "}$, Diploma thesis (1999), University of Heidelberg (unpublished).

[71] W. Bernreuther, A. Brandenburg, P. Haberl and O. Nachtmann, Phys. Lett. B 387 (1996) 155 [arXiv:hep-ph/9606379]; W. Bernreuther and O. Nachtmann, Eur. Phys. J. C 9 (1999) 319 [arXiv:hep-ph/9812259].

[72] U. Baur, I. Hinchliffe and D. Zeppenfeld, Int. J. Mod. Phys. A 2 (1987) 1285; U. Baur, M. Spira and P. M. Zerwas, Phys. Rev. D 42 (1990) 815.

[73] S. Bethke et al., JADE Collaboration, Phys. Lett. B 213 (1988) 235.

[74] V. M. Abazov, D0 Collaboration, Phys. Rev. D 69 (2004) 111101 [hep-ex/0308033].

[75] H. E. Haber and G. L. Kane, Phys. Rept. 117 (1985) 75; J. Gunion, H. E. Haber, G. Kane and S. Dawson, The Higgs Hunter's Guide, Addison-Wesley 1990.

[76] J. R. Ellis, D. V. Nanopoulos and K. A. Olive, Phys. Lett. B 508, 65 (2001) [arXiv:hepph/0102331]; R. Arnowitt, B. Dutta, B. Hu and Y. Santoso, Phys. Lett. B 505 (2001) 177 [arXiv:hep-ph/0102344]; S. Komine, T. Moroi and M. Yamaguchi, Phys. Lett. B 506 (2001) 93 [arXiv:hep-ph/0102204];

[77] S. P. Martin and J. D. Wells, Phys. Rev. D 64 (2001) 035003 [arXiv:hep-ph/0103067].

[78] A. J. Buras, arXiv:hep-ph/9806471; T. Hurth, Rev. Mod. Phys. 75, 1159 (2003) [arXiv:hep-ph/0212304].

[79] I. S. Altarev et al., Phys. Lett. B 276 (1992) 242; I. S. Altarev et al., Phys. Atom. Nucl. 59 (1996) 1152 [Yad. Fiz. 59N7 (1996) 1204]; E. D. Commins, S. B. Ross, D. DeMille and B. C. Regan, Phys. Rev. A 50 (1994) 2960; S. Abel, S. Khalil and O. Lebedev, Nucl. Phys. B 606 (2001) 151 [arXiv:hep-ph/0103320]; V. D. Barger, T. Falk, T. Han, J. Jiang, T. Li and T. Plehn, Phys. Rev. D 64 (2001) 056007 [arXiv:hep-ph/0101106]; S. Oshima, T. Nihei and T. Fujita, arXiv:hep-ph/0501236.

[80] G. Belanger, F. Boudjema, A. Cottrant, A. Pukhov and A. Semenov, arXiv:hepph/0412309; G. Belanger, F. Boudjema, C. Hugonie, A. Pukhov and A. Semenov, arXiv:hep-ph/0505142; M. Drees, arXiv:hep-ph/0410113. 
[81] B. C. Allanach et al., in Proc. of the APS/DPF/DPB Summer Study on the Future of Particle Physics (Snowmass 2001) ed. N. Graf, Eur. Phys. J. C 25 (2002) 113 [eConf C010630 (2001) P125] [arXiv:hep-ph/0202233]. N. Ghodbane and H. U. Martyn, in Proc. of the APS/DPF/DPB Summer Study on the Future of Particle Physics (Snowmass 2001) ed. N. Graf, arXiv:hep-ph/0201233.

[82] G. Moortgat-Pick, arXiv:hep-ph/0410118; C. Blochinger, H. Fraas, G. Moortgat-Pick and W. Porod, Eur. Phys. J. C 24 (2002) 297 [arXiv:hep-ph/0201282]; G. MoortgatPick, arXiv:hep-ph/0410118.

[83] J. L. Feng and M. E. Peskin, Phys. Rev. D 64, 115002 (2001) [arXiv:hep-ph/0105100]; A. Bartl, H. Fraas and W. Majerotto, Z. Phys. C 34, 411 (1987).

[84] Contribution by G. Moortgat-Pick, summer 2004.

[85] A. Freitas, A. von Manteuffel and P. M. Zerwas, Eur. Phys. J. C 34 (2004) 487 [hep$\mathrm{ph} / 0310182]$.

[86] Contribution by A. Freitas, May 2005.

[87] Contribution by T. Abe, S. Chen, B. Dobos, T. Dorland, J. Goodson, J. Gray, A. Han, A. Martinez, U. Nauenberg, J. Proulx, Positron Polarization and Supersymmetry Measurements, November 2004.

[88] A. Bartl, H. Eberl, S. Kraml, W. Majerotto and W. Porod, Eur. Phys. J. directC 2 (2000) 6 [hep-ph/0002115];

[89] Contribution by H. Eberl, S. Kraml, May 2005.

[90] A. Bartl, H. Eberl, S. Kraml, W. Majerotto, W. Porod and A. Sopczak, Z. Phys. C 76 (1997) 549 [arXiv:hep-ph/9701336]; H. Eberl, A. Bartl and W. Majerotto, Nucl. Phys. B 472 (1996) 481 [arXiv:hep-ph/9603206]; S. Kraml, arXiv:hep-ph/9903257.

[91] E. Boos, H. U. Martyn, G. Moortgat-Pick, M. Sachwitz, A. Sherstnev and P. M. Zerwas, Eur. Phys. J. C 30 (2003) 395 [arXiv:hep-ph/0303110].

[92] M. Berggren, R. Keranen, H. Nowak and A. Sopczak, arXiv:hep-ph/9911345.

[93] A. Bartl, H. Fraas and W. Majerotto, Nucl. Phys. B 278 (1986) 1; A. Bartl, H. Fraas, W. Majerotto and B. Mosslacher, Z. Phys. C 55 (1992) 257; T. Tsukamoto, K. Fujii, H. Murayama, M. Yamaguchi and Y. Okada, Phys. Rev. D 51 (1995) 3153; G. Moortgat-Pick and H. Fraas, Phys. Rev. D 59 (1999) 015016 [arXiv:hepph/9708481]; G. Moortgat-Pick, H. Fraas, A. Bartl and W. Majerotto, Eur. Phys. J. C 7 (1999) 113 [arXiv:hep-ph/9804306]. J. L. Kneur and G. Moultaka, Phys. Rev. D 59, 015005 (1999) [arXiv:hep-ph/9807336]; S. Y. Choi, A. Djouadi, H. K. Dreiner, J. Kalinowski and P. M. Zerwas, Eur. Phys. J. C 7 (1999) 123 [arXiv:hep-ph/9806279].

[94] S. Y. Choi, J. Kalinowski, G. Moortgat-Pick and P. M. Zerwas, Eur. Phys. J. C 22 (2001) 563 [Addendum-ibid. C 23 (2002) 769] [arXiv:hep-ph/0108117]; J. Kalinowski, January 2005. 
[95] G. Moortgat-Pick, in Proc. of the APS/DPF/DPB Summer Study on the Future of Particle Physics (Snowmass 2001) ed. N. Graf, eConf C010630 (2001) E3008 [arXiv:hepph/0202082]; G. Moortgat-Pick, A. Bartl, H. Fraas and W. Majerotto, Eur. Phys. J. C 18 (2000) 379 [arXiv:hep-ph/0007222].

[96] Contribution by J. Kalinowski, May 2005.

[97] Contribution by G. Moortgat-Pick, summer 2004.

[98] A. Bartl, H. Fraas, O. Kittel and W. Majerotto, Phys. Rev. D 69 (2004) 035007 [arXiv:hep-ph/0308141]; A. Bartl, H. Fraas, O. Kittel and W. Majerotto, arXiv:hep$\mathrm{ph} / 0308143$.

[99] Y. Kizukuri and N. Oshimo, Phys. Lett. B 249 (1990) 449.

[100] S. Y. Choi, H. S. Song and W. Y. Song, Phys. Rev. D 61 (2000) 075004 [arXiv:hep$\mathrm{ph} / 9907474]$.

[101] A. Bartl, H. Fraas, O. Kittel and W. Majerotto, Eur. Phys. J. C 36 (2004) 233 [arXiv:hep-ph/0402016].

[102] A. Bartl, H. Fraas, O. Kittel and W. Majerotto, Phys. Lett. B 598 (2004) 76 [arXiv:hep$\mathrm{ph} / 0406309]$.

[103] O. Kittel, A. Bartl, H. Fraas and W. Majerotto, Phys. Rev. D 70 (2004) 115005 [arXiv:hep-ph/0410054].

[104] O. Kittel, arXiv:hep-ph/0504183.

[105] A. Bartl, T. Kernreiter and O. Kittel, Phys. Lett. B 578 (2004) 341 [arXiv:hepph/0309340].

[106] S. Y. Choi, M. Drees, B. Gaissmaier and J. Song, Phys. Rev. D 69 (2004) 035008 [arXiv:hep-ph/0310284].

[107] A. Bartl, H. Fraas, S. Hesselbach, K. Hohenwarter-Sodek and G. Moortgat-Pick, JHEP 0408 (2004) 038 [hep-ph/0406190].

[108] A. Bartl, H. Fraas, S. Hesselbach, K. Hohenwarter-Sodek and G. Moortgat-Pick, in preparation.

[109] A. Bartl, H. Fraas, T. Kernreiter, O. Kittel and W. Majerotto, arXiv:hep-ph/0310011.

[110] G. Moortgat-Pick, H. Fraas, A. Bartl and W. Majerotto, Eur. Phys. J. C 9 (1999) 521 [Erratum-ibid. C 9 (1999) 549] [arXiv:hep-ph/9903220].

[111] A. Bartl, K. Hohenwarter-Sodek, T. Kernreiter and H. Rud, Eur. Phys. J. C 36 (2004) 515 [hep-ph/0403265].

[112] A. Bartl, H. Fraas, S. Hesselbach, K. Hohenwarter-Sodek, T. Kernreiter G. MoortgatPick, in preparation. 
[113] P. Chiappetta, J. Soffer, P. Taxil, F.M. Renard, P. Sorba, Nucl. Phys. B 262495 (1985) [Erratum B 279, 824 (1987)].

[114] S. Y. Choi, A. Djouadi, M. Guchait, J. Kalinowski, H. S. Song and P. M. Zerwas, Eur. Phys. J. C 14 (2000) 535 [arXiv:hep-ph/0002033].

[115] F. Franke and H. Fraas, Int. J. Mod. Phys. A 12 (1997) 479 [arXiv:hep-ph/9512366] and references therein.

[116] F. Franke, H. Fraas and A. Bartl, Phys. Lett. B 336 (1994) 415 [arXiv:hepph/9408217].

[117] F. Franke and H. Fraas, Z. Phys. C 72 (1996) 309 [arXiv:hep-ph/9511275].

[118] U. Ellwanger and C. Hugonie, Eur. Phys. J. C 5 (1998) 723 [arXiv:hep-ph/9712300].

[119] D. J. Miller, R. Nevzorov and P. M. Zerwas, Nucl. Phys. B 681 (2004) 3 [arXiv:hepph/0304049]; S. Y. Choi, D. J. Miller and P. M. Zerwas, Nucl. Phys. B 711 (2005) 83 [arXiv:hep-ph/0407209].

[120] J. F. Gunion and M. Szleper, arXiv:hep-ph/0409208.

[121] U. Ellwanger, J.-F. Gunion and C. Hugonie, arXiv:hep-ph/0503203.

[122] J. L. Hewett and T. G. Rizzo, Z. Phys. C 36, 209 (1987); J. L. Hewett and T. G. Rizzo, Phys. Rept. 183, 193 (1989).

[123] S. Hesselbach, F. Franke and H. Fraas, Eur. Phys. J. C 23 (2002) 149 [arXiv:hep$\mathrm{ph} / 0107080]$ and references therein.

[124] F. Franke and S. Hesselbach, Phys. Lett. B 526 (2002) 370 [arXiv:hep-ph/0111285].

[125] S. Hesselbach and F. Franke, arXiv:hep-ph/0210363.

[126] G. Moortgat-Pick, S. Hesselbach, F. Franke and H. Fraas, arXiv:hep-ph/9909549.

[127] S. Hesselbach, F. Franke and H. Fraas, arXiv:hep-ph/0003272.

[128] B. de Carlos and J. R. Espinosa, Phys. Lett. B 407 (1997) 12 [arXiv:hep-ph/9705315].

[129] G. Moortgat-Pick, S. Hesselbach, F. Franke and H. Fraas, arXiv:hep-ph/0502036 .

[130] L. J. Hall and M. Suzuki, Nucl. Phys. B 231 (1984) 419; S. Dawson, Nucl. Phys. B 261 (1985) 297.

[131] B. C. Allanach, A. Dedes and H. K. Dreiner, Phys. Rev. D 69, 115002 (2004) [arXiv:hep-ph/0309196]; B. C. Allanach, A. Dedes and H. K. Dreiner, Phys. Rev. D 60, 075014 (1999) [arXiv:hep-ph/9906209]; H. K. Dreiner, arXiv:hep-ph/9707435; A. Faessler, T. S. Kosmas, S. Kovalenko and J. D. Vergados, Nucl. Phys. B 587, 25 (2000). 
[132] H. K. Dreiner and S. Lola, Prepared for Physics with e+e-Linear Colliders (The European Working Groups 4 Feb - 1 Sep 1995: Session 3), Hamburg, Germany, 30 Aug - 1 Sep 1995 B. C. Allanach, H. K. Dreiner, P. Morawitz and M. D. Williams, Phys. Lett. B 420 (1998) 307 [arXiv:hep-ph/9708495]; H. K. Dreiner, P. Richardson and M. H. Seymour, arXiv:hep-ph/9903419;

[133] J. Kalinowski, R. Ruckl, H. Spiesberger and P. M. Zerwas, Phys. Lett. B 406, 314 (1997) [arXiv:hep-ph/9703436]; M. Heyssler, R. Ruckl and H. Spiesberger, arXiv:hepph/9908319.

[134] Contribution by H. Spiesberger, May 2005.

[135] T. G. Rizzo, Phys. Rev. D 59 (1999) 113004 [arXiv:hep-ph/9811440].

[136] H. K. Dreiner and S. Lola, OUTP-92-02-P M. Chemtob and G. Moreau, Phys. Rev. D 59 (1999) 055003 [arXiv:hep-ph/9807509]. G. Moreau, arXiv:hep-ph/0009140.

[137] T. Hahn, S. Heinemeyer and G. Weiglein, Nucl. Phys. B 652 (2003) 229 [arXiv:hepph/0211204]; T. Hahn, S. Heinemeyer and G. Weiglein, Nucl. Phys. Proc. Suppl. 116 (2003) 336 [arXiv:hep-ph/0211384].

[138] E. Eichten, K. D. Lane and M. E. Peskin, Phys. Rev. Lett. 50 (1983) 811; R. Ruckl, Phys. Lett. B 129 (1983) 363.

[139] B. Schrempp, F. Schrempp, N. Wermes and D. Zeppenfeld, Nucl. Phys. B 296 (1988) 1.

[140] A. A. Babich, P. Osland, A. A. Pankov and N. Paver, Phys. Lett. B 518 (2001) 128 [arXiv:hep-ph/0107159]; A. A. Babich, P. Osland, A. A. Pankov and N. Paver, October 2003.

[141] A. A. Babich, P. Osland, A. A. Pankov and N. Paver, LC-TH-2001-021, arXiv:hep$\mathrm{ph} / 0101150$.

[142] A. A. Babich, P. Osland, A. A. Pankov and N. Paver, Phys. Lett. B 481 (2000) 263 [arXiv:hep-ph/0003253].

[143] C. J. S. Damerell and D. J. Jackson, eConf C960625 (1996) DET078.

[144] S. Riemann, LC-TH-2001-007.

[145] A. A. Pankov and N. Paver, Eur. Phys. J. C 29 (2003) 313 [arXiv:hep-ph/0209058].

[146] A. Leike, Phys. Rept. 317, 143 (1999) [arXiv:hep-ph/9805494]; M. Cvetic and S. Godfrey, arXiv:hep-ph/9504216.

[147] F. Cuypers, eConf C960625 (1996) NEW137 [arXiv:hep-ph/9611336];

[148] R. Casalbuoni, S. De Curtis, D. Dominici, R. Gatto and S. Riemann, LC-TH-2000006, arXiv:hep-ph/0001215.

[149] B. Ananthanarayan and S. D. Rindani, Phys. Rev. D 70, 036005 (2004) [arXiv:hepph/0309260]; Phys. Lett. B 606, 107 (2005) [arXiv:hep-ph/0410084]; 
[150] S. D. Rindani, Phys. Lett. B 602 (2004) 97 [arXiv:hep-ph/0408083].

[151] R. Haas, Fermilab-Conf-00/270E CDF (2000); S.M. Wang, hep-ex/0405075.

[152] W. Buchmüller and D. Wyler, Phys. Lett. B 177, 377 (1986).

[153] M. Tanabashi in S. Eidelman et al. (Particle Data Group), Phys. Lett. B 592, 1 (2004).

[154] C.Q. Geng, Zeit. Phys. C 48, 279 (1990); X.-G. He, B.H.J. McKellar and S. Pakvasa, Phys. Lett. B 283, 348 (1992); S. Davidson, D. Bailey and B.A. Campbell, Zeit. Phys. C 61, 613 (1994); G. Bhattacharyya, J. Ellis and K. Sridhar, Phys. Lett. B 336, 100 (1994); 338, 522 (1994) (E); G. Bhattacharyya, D. Choudhury and K. Sridhar, Phys. Lett. B 349, 118 (1995); J.K. Mizukoshi, O.J.P. Éboli and M.C. Gonzalez-Garcia, Nucl. Phys. B 443, 20 (1995); O.J.P. Éboli, IFUSP 1170, hep-ph/9508342; W. Bernreuther, A. Brandenburg and P. Overmann, Phys. Lett. B 391, 413 (1997). P. Poulose and S.D. Rindani, Pramana - J. Phys. 51, 387 (1998);

[155] P. Herczeg, Phys. Rev. D 68 (2003) 116004 [Erratum-ibid. D 69 (2004) 039901].

[156] I. Antoniadis, Phys. Lett. B 246, 377 (1990); N. Arkani-Hamed, S. Dimopoulos and G. R. Dvali, Phys. Lett. B 429, 263 (1998) [arXiv:hep-ph/9803315]; I. Antoniadis, N. Arkani-Hamed, S. Dimopoulos and G. R. Dvali, Phys. Lett. B 436, 257 (1998) [arXiv:hep-ph/9804398]; N. Arkani-Hamed, S. Dimopoulos and G. R. Dvali, Phys. Rev. D 59, 086004 (1999) [arXiv:hep-ph/9807344].

[157] G. F. Giudice, R. Rattazzi and J. D. Wells, Nucl. Phys. B 544 (1999) 3 [arXiv:hep$\mathrm{ph} / 9811291]$

[158] T. Han, J. D. Lykken and R. J. Zhang, Phys. Rev. D 59 (1999) 105006 [arXiv:hep$\mathrm{ph} / 9811350]$

[159] K. Cheung, arXiv:hep-ph/0409028; J. Hewett and M. Spiropulu, Ann. Rev. Nucl. Part. Sci. 52 (2002) 397 [hep-ph/0205106].

[160] G. W. Wilson, "Study of the sensitivity to extra dimensions in the photon graviton channel with polarised beams," LC-PHSM-2001-010.

[161] L. Vacavant and I. Hinchliffe, arXiv:hep-ex/0005033.

[162] L. Randall and R. Sundrum, Phys. Rev. Lett. 83 (1999) 3370 [arXiv:hep-ph/9905221].

[163] J. L. Hewett, Phys. Rev. Lett. 82 (1999) 4765 [arXiv:hep-ph/9811356]; T. G. Rizzo, Phys. Rev. D 59 (1999) 115010 [arXiv:hep-ph/9901209].

[164] S. Cullen, M. Perelstein and M. E. Peskin, Phys. Rev. D 62 (2000) 055012 [arXiv:hep$\mathrm{ph} / 0001166]$.

[165] For review on RD phenomenology see e. g. H. Davoudiasl, J. L. Hewett and T. G. Rizzo, Phys. Lett. B 473, 43 (2000) [arXiv:hep-ph/9911262]; Phys. Rev. Lett. 84, 2080 (2000) [arXiv:hep-ph/9909255]; J. L. Hewett, F. J. Petriello and T. G. Rizzo, JHEP 0209, 030 (2002) [arXiv:hep-ph/0203091]. 
[166] T. G. Rizzo, JHEP 0210 (2002) 013 [arXiv:hep-ph/0208027].

[167] P. Osland, A. A. Pankov and N. Paver, Phys. Rev. D 68 (2003) 015007 [arXiv:hepph/0304123]; A. A. Pankov and N. Paver, hep-ph/0501170.

[168] A. Datta, E. Gabrielli and B. Mele, Phys. Lett. B 552 (2003) 237 [arXiv:hep$\mathrm{ph} / 0210318]$.

[169] T. G. Rizzo, JHEP 0302, 008 (2003) [arXiv:hep-ph/0211374]; JHEP 0308, 051 (2003) [arXiv:hep-ph/0306283].

[170] H. A. Olsen, P. Osland and I. Overbo, Phys. Lett. B 97 (1980) 286.

[171] D. Choudhury and S. D. Rindani, Phys. Lett. B 335 (1994) 198 [arXiv:hep$\mathrm{ph} / 9405242]$.

[172] B. Ananthanarayan, S. D. Rindani, R. K. Singh and A. Bartl, Phys. Lett. B 593 (2004) 95 [arXiv:hep-ph/0404106].

[173] B. Ananthanarayan and S. D. Rindani, Phys. Lett. B 606 (2005) 107 [arXiv:hep$\mathrm{ph} / 0410084]$.

[174] H. Czyz, K. Kolodziej and M. Zralek, Z. Phys. C 43 (1989) 97.

[175] S. D. Rindani and J. P. Singh, Phys. Lett. B 419 (1998) 357 [arXiv:hep-ph/9703380].

[176] S. D. Rindani, Pramana 45 (1995) S263 [arXiv:hep-ph/9411398].

[177] K. J.Abraham, B.Lampe, Phys. Lett. B 326175 (1994).

[178] K. Floettmann, EPAC-2004-TUZACH01 Presented at the 9th European Particle Accelerator Conference (EPAC 2004), Lucerne, Switzerland, 5-9 Jul 2004; K. Flottmann, Investigations toward the development of polarized and unpolarized high intensity positron sources for linear colliders, PhD Thesis, DESY-93-161.

[179] Alexander Mikhailichenko, Why Polarized Positrons should be in the Base Line of Linear Collider, Cormell, CLNS-04-1894, Nov 2004. 18pp.

http://www.lns.cornell.edu/public/CLNS/2004/CLNS04-1894/CLNS04-1894.pdf

[180] U.S. Linear Collider Steering Group, Accelerator Sub-committee, Linear Collider Option Task Forces, U.S. Linear Collider Technology Options Study, March 2004.

[181] D.P. Barber, G. Ripken, Handbook of Accelerator Physics and Engineering, Eds. A.W. Chao, M. Tigner, World Scientific, 2nd edition (2002).

[182] D.P. Barber and W. Decking, Polarization Preservation in the NLC and TESLA Damping Rings, Hamburg, August 2002; ILC-TRC, International Linear Collider Technical Review Committee Second Report 2003, SLAC-R-606, webpage: http://www.slac.stanford.edu/xorg/ilc-trc/2002/2002/report/03rephome.htm.

[183] R.W. Assmann, F. Zimmermann, CLIC Note 501 (2001), CERN SL-2001-064.

[184] K. Yokoya and P. Chen. SLAC-PUB-4692, 1988. 
[185] Kathleen A. Thompson, SLAC-PUB-8716, 2001.

[186] K. Yokoya, CAIN, http://www-acc-theory.kek.jp/members/cain/default.html

[187] V.E. Balakin and A.A. Mikhailichenko, The Conversion System for Obtaining High Polarized Electrons and Positrons, Budker Institute of Nuclear Physics, Preprint BINP 79-85 (1979). The original paper is difficult to get, Mikhailichenko's Thesis, to which it lead, has been translated by him:

http://www.lns.cornell.edu/public/CBN/2002/CBN02-13/DISSERT.pdf

T. A. Vsevolozhskaya, A. A. Mikhailichenko, E. A. Perevedentsev, G. I. Silvestrov and A. D. Chernyakin, SLAC-TRANS-0225 Presented at Int. Conf. In High Energy Accelerators, Novosibirsk, USSR, Aug 7-11, 1986.

[188] A. Mikhailichenko and T. Moore, CBN-02-6; A. Mikhailichenko, CBN-03-4; A. Mikhailichenko, Proceedings of Workshop on Superconducting Undulators and Wiggglers, Grenoble, June (2003), see http:/ / www.esrf.fr/Accelerators / files/Conferences/mikhailichenko.pdf

[189] H. Olsen, L.C. Maximom, Photon and Electron Polarization in High-Energy Bremsstrahlung and Pair Production with Screening, Phys. Rev. 114, (1959) 877.

[190] TESLA Technical Design Report, 2001, Chap. 4, http://tesla.desy.de/new_pages/TDR_CD/PartII/chapter04/chapter04.pdf

[191] G. Alexander et al., E-166 collaboration, SLAC-TN-04-018, LC-DET-2003-044.

[192] John P. Blewett and R. Chasman, Orbits and fields in the helical wiggler, J. Appl. Phys. 48,2692(1977).

[193] D.J. Scott, et al., Design Considerations for a Helical Undulator for the Production of Polarised Positrons for TESLA, EPAC 2004.

http://www.astec.ac.uk/lc-abd/epac2004/mopkf064.pdf

[194] J.M.J. Madey, J. Appl. Phys. V42,1906(1971).

[195] K. Halbach, Nucl. Instr. Meth. A169,1(1980).

[196] 1 T. Omori, contribution to the 1st ILC Workshop, Nov.13-15, 2004, KEK, Tsukuba, see http://lcdev.kek.jp/ILCWS/Talks/13wg3-6-WG306_Positron_Discussion_Omori.pdf

[197] T. Omori, T. Aoki, K. Dobashi, T. Hirose, Y. Kurihara, T. Okugi, I. Sakai, A. Tsunemi, J. Urakawa, M. Washio, K. Yokoya, Nucl. Instrum. Meth. A 500 (2003) 232 [Erratumibid. A 503 (2003) 658].

[198] S. Hiramatsu, contribution to the 11th KEK-SLAC ISG Meeting Dec.16-19, 2003, KEK, Tsukuba, http:/ /lcdev.kek.jp/ISG/ISG11/Source/10miconFEL.pdf.

[199] private communication with K. Moenig.

[200] private communication with R. Pitthan. 
[201] I. Sakai et al., Phys. Rev. ST Accel. Beams 6 (2003) 091001.

[202] M. Fukuda, T. Aoki, K. Dobashi, T. Hirose, T. Iimura, Y. Kurihara, T. Okugi, T. Omori, I. Sakai, J. Urakawa, and M. Washio, Phys. Rev. Lett 91, 164801 (2003).

[203] T. Nakanishi, H. Aoyagi, H. Horinaka, Y. Kamiya, T. Kato, S. Nakamura, T. Saka and M. Tsubata, Phys. Lett. A 1581991), 345-349.

[204] T. Maruyama, E.L. Garwin, G.H. Zapalac, J.S. Smith and J.D. Walker, Phys. Rev. Lett. 66 (1991) 2376

[205] T. Omori, Y. Kurihara, T. Nakanishi, H. Aoyagi, T. Baba, T. Furuya, K. Itoga, M. Mizuta, S. Nakamura, Y. Takeuchi, M. Tsubata, and M. Yoshioka, Phys. Rev. Lett. 67 (1991) 3294-3297; T. Omori, Y. Kurihara, Y. Takeuchi, M. Yoshioka, T. Nakanishi, S. Okumi, M. Tsubata, M. Tawada, K. Togawa, Y. Tanimoto, C. Takahashi, T. Baba, and M. Mizuta, Jpn. J. Appl. Phys. 33 (1994) 5676-5680.

[206] Y. Kurihara, T. Omori, T. Nakanishi, H. Aoyagi, T. Baba, T. Furuya, K. Itoga, M. Mizuta, S. Nakamura, Y. Takeuchi, M. Tsubata and M. Yoshioka, Nucl. Instr. Meth. A 313 (1992) 393-397.

[207] T. Omori, Y. Kurihara, K. Itoga, Y. Takeuchi, M. Yoshioka, T. Nakanishi, H. Aoyagi, M. Tsubata, S. Nakamura, T. Baba, and M. Mizuta, Int. J. Mod. Phys. A, Proc. Suppl. 2A (1993) 157-159; K. Togawa, T. Nakanishi, T. Baba, F. Furuta, H. Horinaka, T. Ida, Y. Kurihara, H. Matsumoto, T. Matsuyama, M. Mizuta, S. Okumi, T. Omori, C. Suzuki, Y. Takeuchi, K. Wada, K. Wada and M. Yoshioka, Nucl. Instr. and Meth. in Phys. Res. A414 (1998) p431-445.

[208] T. Nakanishi, K. Togawa, T. Baba, F. Furuta, H. Horinaka, T. Kato, Y. Kurihara, H. Matsumoto, T. Matsuyama, T. Nishitani, S. Okumi, T. Omori, T. Saka, C. Suzuki, Y. Takeuchi, K. Wada, K. Wada, M. Yamamoto, M. Yoshioka, Nucl. Instrum. Meth. A455 (2000) 109-112.

[209] R. Prepost and T. Maruyama, Annu. Rev. Nucl. Part. Sci. 45, 41 (1995).

[210] T. Nishitani, T. Nakanishi, M. Yamamoto et al., J. Appl. Phys. 97, 094907 (2005).

[211] T. Maruyama, D.A. Luh, A. Brachmann et al., Appl. Phys. Lett. 85, 2640 (2004).

[212] P. L. Anthony, R. G. Arnold, C. Arroyo et al., SLAC-PUB-11149, 2005, hepex/0504049.

[213] T. Maruyama, A. Brachmann, J. E. Clendenin et al., Nucl. Instrum. and Meth. A 492, 199 ( 2002).

[214] P. L. Anthony, R. G. Arnold, C. Arroyo et al., Phys. Ref. Lett. 92, 181602 (2004).

[215] K. Moffeit, M. Woods, P. Schüler, K. Moenig, Ph. Bambade, Spin Rotation Schemes at the ILC for Two Interaction Regions and Positron Polarization with two Helicities, SLACTN-05-045, LCC-0159, IPBI TN-2005-2.

[216] P. Emma, A Spin Rotator System for the NLC, NLC Note 7, December, 1994. 
[217] M. L. Swartz, Phys. Rev. D 58 (1998) 014010 [arXiv:hep-ph/9711447].

[218] J.D. Ullman, H. Frauenfelder, H.J. Lipkin, A. Rossi, "Determination of Electron and Positron Helicity with Möller and Bhabha Scattering", Phys. Rev. 122 (1961) 536.

[219] H. Frauenfelder and A. Rossi, in Methods of Experimental Physics, edited by L.C.L. Yuan and C.S. Wu (Academic, New York, 1963) Vol. 5, part B, p. 214-274.

[220] F. Corriveau et al., Phys. Rev. D24 (1981) 2004.

[221] P.S. Cooper et al., Phys. Rev. Lett.34 (1975) 1589.

[222] C.Y. Prescott et al., Phys. Lett. B77 (1978) 347; Phys. Lett. B84 (1979) 524.

[223] G. Baum et al., Phys. Rev. Lett. 51 (1983) 1135.

[224] M. Swartz et al., Nucl. Instr. and Meth. A363 (1995) 526.

[225] H.R. Band et al., Nucl. Instr. and Meth. A400 (1997) 24.

[226] P. Steiner et al., Nucl. Instr. and Meth. A419 (1998) 105.

[227] B. Wagner et al., Nucl. Instr. and Meth. A294 (1990) 541.

[228] J. Arrington et al., Nucl. Instr. and Meth. A311 (1992) 39.

[229] D. Adams et al., Nucl. Instr. and Meth. A443 (2000) 1.

[230] M. Hauger et al., Nucl. Instr. and Meth. A462 (2001) 382.

[231] L.V. de Bever et al., Nucl. Instr. and Meth. A400 (1997) 379.

[232] G. Alexander, I. Cohen, Nucl. Instr. and Meth. A486 (2002) 552.

[233] A. Airapetian et al., Nucl. Instr. and Meth. A540 (2005) 68.

[234] Thomas Benisch, PhD-Thesis, University of Erlangen-Nürnberg, 1998.

[235] V. Gharibyan, N. Meyners and P. Schuler, LC-DET-2001-047; see http:/ /www.desy.de/ Icnotes.

[236] M. Woods, K. C. Moffeit, T. O. Raubenheimer, A. Seryi, C. Sramek and A. Florimonte, SLAC-PUB-10353, 2004, arXiv:physics/0403037.

[237] M. Woods and K. C. Moffeit, SLAC-PUB-10669,SLAC IPBI TN-2004-3, 2004, Presented at International Conference on Linear Colliders (LCWS 04), Paris, France, 19-24 Apr 2004

[238] V. Brisson, R. Chiche, M. Jaquet-Lemire, S. Kurbasov, L. Losev, C. Pascaud, A. Reboux, V. Soskov, Z. Zhang, F. Zomer, Paris, August 2004.

[239] N. Falletto et al., Nucl. Instrum. Meth. A 459 (2001) 412.

[240] M. S. Bilenky, J. L. Kneur, F. M. Renard and D. Schildknecht, Nucl. Phys. B 409 (1993) 22. 
[241] K. Monig, LC-PHSM-2000-059.

[242] S. Jadach, B. F. L. Ward and Z. Was, Comput. Phys. Commun. 130 (2000) 260 [arXiv:hep-ph/9912214].

[243] F. Cuypers and P. Gambino, Phys. Lett. B 388 (1996) 211 [hep-ph/9606391];

[244] A.M. Bincer, Phys. Rev. 107 (1957) 1434.

[245] G.W. Ford, C.J. Mullin, Phys. Rev.108 (1957) 477.

[246] A.A. Kresnin, L.N. Rozentsveig, Journ. Exp. Theor. Phys. 32 (1957) 353 [Soviet Physics JETP 5 (1957) 288].

[247] G. Alexander, E. Reinherz-Aronis, Measurement of low energy longitudinal polarised positron beams via a Bhabha polarimeter, TAUP-2791-05, hep-ex/0505001.

[248] P. Chen, T. Ohgaki, A. Spitkovsky, T. Takahashi and K. Yokoya, Nucl. Instrum. Meth. A 397 (1997) 458 [arXiv:physics/9704012].

[249] G. Corcella et al., JHEP 0101 (2001) 010 [arXiv:hep-ph/0011363]; G. Corcella et al., arXiv:hep-ph/0210213.

[250] H. Baer, F. E. Paige, S. D. Protopopescu and X. Tata, arXiv:hep-ph/0001086.

[251] T. Sjöstrand, P. Edén, C. Friberg, L. Lönnblad, G. Miu, S. Mrenna and E. Norrbin, Comput. Phys. Commun. 135 (2001) 238 [arXiv:hep-ph/0010017]; T. Sjöstrand, L. Lönnblad and S. Mrenna, arXiv:hep-ph/0108264.

[252] P. Richardson, JHEP 0111 (2001) 029 [arXiv:hep-ph/0110108].

[253] R. Kuhn, F. Krauss, B. Ivanyi and G. Soff, Comput. Phys. Commun. 134 (2001) 223 [arXiv:hep-ph/0004270]; SHERPA is available from:

http://www.physik.tu-dresden.de/rkrauss/hep/.

[254] M. Bertini, L. Lönnblad and T. Sjöstrand, Comput. Phys. Commun. 134 (2001) 365 [arXiv:hep-ph/0006152].

[255] S. Gieseke, arXiv:hep-ph/0210294.

[256] M. Kobel et al. [Two Fermion Working Group], arXiv:hep-ph/0007180.

[257] M. W. Grunewald et al., arXiv:hep-ph/0005309.

[258] S. Dittmaier and M. Roth, Nucl. Phys. B 642 (2002) 307 [arXiv:hep-ph/0206070].

[259] G. Montagna, M. Moretti, O. Nicrosini and F. Piccinini, Eur. Phys. J. C 2 (1998) 483 [arXiv:hep-ph/9705333]; F. Gangemi, G. Montagna, M. Moretti, O. Nicrosini and F. Piccinini, Eur. Phys. J. C 9 (1999) 31 [arXiv:hep-ph/9811437]; F. Gangemi, G. Montagna, M. Moretti, O. Nicrosini and F. Piccinini, Nucl. Phys. B 559 (1999) 3 [arXiv:hepph/9905271]; F. Gangemi, arXiv:hep-ph/0002142. 
[260] K. Kołodziej, Eur. Phys. J. C 23 (2002) 471 [arXiv:hep-ph/0110063]; K. Kołodziej, Comput. Phys. Commun. 151 (2003) 339 [arXiv:hep-ph/0210199]; A. Biernacik and K. Kołodziej, Nucl. Phys. Proc. Suppl. 116 (2003) 33 [arXiv:hep-ph/0210405].

[261] E. Accomando, A. Ballestrero and M. Pizzio, Nucl. Phys. B 547 (1999) 81 [arXiv:hepph/9807515]; E. Accomando, A. Ballestrero and M. Pizzio, arXiv:hep-ph/9709277.

[262] F. Krauss, R. Kuhn and G. Soff, JHEP 0202 (2002) 044 [arXiv:hep-ph/0109036].

[263] A. Pukhov et al., "CompHEP: A package for evaluation of Feynman diagrams and integration over multi-particle phase space. User's manual for version 33," arXiv:hep-ph/9908288. COMPHEP is available from:

http://theory.sinp.msu.ru/comphep/.

[264] T. Ishikawa, T. Kaneko, K. Kato, S. Kawabata, Y. Shimizu and H. Tanaka [MINAMITATEYA group], KEK-92-19. GRACE is available from:

http://minami-home.kek.jp/.

[265] A. Kanaki and C. G. Papadopoulos, Comput. Phys. Commun. 132 (2000) 306 [arXiv:hep-ph/0002082].

[266] C. G. Papadopoulos, Comput. Phys. Commun. 137 (2001) 247 [arXiv:hep$\mathrm{ph} / 0007335]$.

[267] F. Maltoni and T. Stelzer, JHEP 0302 (2003) 027 [arXiv:hep-ph/0208156]; T. Stelzer and W. F. Long, Comput. Phys. Commun. 81 (1994) 357 [arXiv:hep-ph/9401258]. MADGRAPH/MADEVENT is available from: http://madgraph.physics.uiuc.edu/.

[268] H. Murayama, I. Watanabe and K. Hagiwara, KEK-91-11.

[269] T. Ohl, "O'Mega and WHIZARD: Monte Carlo event generator generation for future colliders," arXiv:hep-ph/0011287; W. Kilian, LC-TOOL-2001-039. In '2nd ECFA/DESY Study 1998-2001', 1924-1980. WHI ZARD is available from:

http://www-ttp.physik.uni-karlsruhe.de/Progdata/whizard/.

For $O^{\prime} M E G A$, see:

http://heplix.ikp.physik.tu-darmstadt.de/rohl/omega/.

[270] F. Caravaglios and M. Moretti, Phys. Lett. B 358 (1995) 332 [arXiv:hep-ph/9507237].

[271] M. Moretti, T. Ohl and J. Reuter, “O'Mega: An optimizing matrix element generator," arXiv:hep-ph/0102195.

[272] N. Ghodbane, arXiv:hep-ph/9909499; S. Katsanevas and P. Morawitz, Comput. Phys. Commun. 112 (1998) 227 [arXiv:hep-ph/9711417]. SUSYGEN is available from: http://lyoinfo.in2p3.fr/susygen/susygen3.html. 